\title{
The bees of the genus Hylaeus FABRICIUS, 1793 of Turkey, with keys to the subgenera and species (Hymenoptera: Anthophila, Colletidae)
}

\author{
With 17 figures
}

HikMet ÖZBeK ${ }^{1}$ and Holger H. DAthe ${ }^{2}$

${ }^{1}$ Atatürk University, Agricultural Faculty, Plant Protection Department, TR-25240 Erzurum, Turkey. - hozbek@atauni.edu.tr
${ }^{2}$ Senckenberg Deutsches Entomologisches Institut, Eberswalder Straße 90, 15374 Müncheberg, Germany.
- holger.dathe@senckenberg.de
Published on 2020-12-01

DOI:10.21248/contrib.entomol.70.2.273-346

\begin{abstract}
The paper presents data of around 4000 previously unpublished specimens, collected in various parts of the country during the last decades. With literature sources, a total of 86 species of the genus Hylaeus FABRICIUs, 1793 from 10 subgenera are compiled for Turkey. New for Turkey are 11 species: Hylaeus (Dentigera) kahri Förster, 1871, H. (Dentigera) pallidicornis Morawitz, 1876, H. (Hylaeus) deceptorius (Benoist, 1959), H. (Hylaeus) gracilicornis (Morawitz, 1867), H. (Hylaeus) paulus Bridwell, 1919, H. (Hylaeus) trisignatus Morawitz, 1876, H. (Nesoprosopis) pectoralis Förster, 1871, H. (Prosopis) incongruus Förster, 1871, H. (Prosopis) trinotatus (PÉrez, 1896), H. (Prosopis) variolaris Morawitz, 1876 and $H$. (Spatulariella) sulphuripes (Gribodo, 1894). No new specimens could be found of 13 species which had been detected formerly. Our knowledge on the distribution of numerous species is greatly expanded. The characteristics of distribution are defined for the individual species. For example, $H$. meridionalis is the most widespread with records from 46 provinces covering all geographical regions of the country, while many other species are only known from one province, sometimes from a single record. The eastern part of Turkey, in particular the province of Hakkâri, proved to be an important centre of diversity for Hylaeus species. For a number of species the valid names had to be revised. Distribution maps are presented for the newly recorded and rare species. Frequently visited plant species are mentioned. Keys to the subgenera and species of Turkish Hylaeus are provided.
\end{abstract}

\section{Nomenclatural acts}

All species described by WARNCKE in 1981 and 1992 from Turkey in the genus Prosopis F. have not yet been revised and formally combined with the valid genus name Hylaeus F. There are however a number of online checklists, which have already registered most of these names under Hylaeus. Representatives of these checklists were checked here: The World Bee Checklist, integrated into the ITIS database (ITIS 2020) and Discover Life (AsCHER \& PICKERING 2019). As new combinations, only the missing names are added here.

Hylaeus (Dentigera) giresunus (WARNCKE, 1992) - comb. et stat. nov.

Hylaeus (Hylaeus) trochilus (WARNCKE, 1992) - comb. et stat. nov.

Hylaeus (Paraprosopis) decaocto (WARNCKE, 1992) - comb. nov.

Hylaeus (Paraprosopis) socheri DATHe, $2010=$ H. (Paraprosopis) decaocto (WARNCKe, 1992) - syn. nov.

Prosopis styriaca crecca WARNCKE, 1992 = Hylaeus (Paraprosopis) styriacus FöRSTER, 1871 - stat. nov. 
Hylaeus (Prosopis) rubosus (WARNCKe, 1981) - comb. nov. Hylaeus (Spatulariella) planulus (WARNCKe, 1981) - comb. nov. Hylaeus (Spatulariella) tauricus WARNCKE, 1981 - comb. nov.

\section{Key words}

Apoidea, taxonomy, distribution data, flower visits, nomenclature, stat. nov., comb. nov.

\section{Zusammenfassung}

Daten von rund 4000 bisher unveröffentlichten Aufsammlungen der letzten Jahrzehnte aus 40 türkischen Provinzen wurden ausgewertet. Einschließlich der Literaturquellen werden für die Türkei insgesamt 86 Arten der Gattung Hylaeus FABRICIUS, 1793 aus 10 Untergattungen zusammengestellt. Neu für die Türkei sind 11 Arten: Hylaeus (Dentigera) kahri Förster, 1871, H. (Dentigera) pallidicornis Morawitz, 1876, H. (Hylaeus) deceptorius (Benoist, 1959), H. (Hylaeus) gracilicornis (Morawitz, 1867), H. (Hylaeus) paulus Bridwell, 1919, H. (Hylaeus) trisignatus Morawitz, 1876, H. (Nesoprosopis) pectoralis Förster, 1871, H. (Prosopis) incongruus Förster, 1871, H. (Prosopis) trinotatus (PÉrez, 1896), H. (Prosopis) variolaris Morawitz, 1876 und H. (Spatulariella) sulphuripes (Gribodo, 1894). Für 13 Arten, die schon früher nachgewiesen waren, konnten keine neuen Belege gefunden werden. Unser Kenntnisstand über die Verbreitung zahlreicher Arten wird erheblich erweitert. Für die einzelnen Arten wird eine Verbreitungscharakteristik definiert. Zum Beispiel ist $H$. meridionalis die am weitesten verbreitete Art mit Nachweisen aus 46 Provinzen, die alle geografischen Regionen des Landes abdecken. Andere Arten sind nur aus einer Provinz bekannt, manchmal mit einem einzigen Datensatz. Der östliche Teil der Türkei, insbesondere die Provinz Hakkâri, erweist sich als ein wichtiges Diversitäts-Zentrum für Hylaeus-Arten. Für eine Anzahl von Arten mussten die gültigen Namen revidiert werden. Für die neu festgestellten und die seltenen Arten wurden Verbreitungskarten erstellt. Häufig besuchte Pflanzenarten sind erwähnt. Außerdem werden Bestimmungstabellen für die Untergattungen und die Arten der Türkei gegeben.

\section{Introduction}

The bee family Colletidae has a worldwide distribution. Nearly all members of the family are characterized by glossal features not found in the other bee families: the glossa is short, commonly broader than long, truncated. Although Colletidae are morphologically diverse bees in the Palearctic region there are only two common genera, Colletes Latreille, 1802 and Hylaeus Fabricius, 1793 (Michener 2007). The genus Hylaeus is widespread globally and makes up a smaller but not negligible share of the total bee fauna. In the Holarctic region, it is easily recognized by the combination of the following characters: size minute to small, body slender (hylaeiform), often almost hairless, black or rarely partly red, usually with limited yellow or white integument markings on the head and mesosoma. In most of the males, the paraocular areas, the clypeus and the supraclypeal area are entirely pale (white or yellow) (Michener 2007; Proshchalykin \& Dathe 2016).

Estimates of the number of species in the genus are still highly divergent. Michener (2007) identified about 630 known species from the literature, while the list of Ascher \& Pickering (2019) gives 768 names. These are assigned to 54 subgenera. In the Palearctic region more than 200 species occur, with centres of diversity in the Mediterranean area and in Central Asia (Michener 2007; Dathe 2015; Proshchalykin \& Dathe 2017).

Many species of Hylaeus nest in dead stems, making rows of cells out of cellophane-like material. Some species, however, make their cells alternatively or regu- larly in various small cavities. Due to the lack of a scopa, pollen is normally carried in the crop along with liquid, presumably nectar. This is not a primitive but a derived characteristic, as has only been recognised in recent years. The issue is discussed in detail by Michener (2007: $88-92)$. The provision in cells is often liquid, and the egg floats on the surface of the provisions (MiCHENER 2007). In-depth studies on the bionomy of individual species can be found in JANVIER (2012), a current compilation is provided by WeSTRICH (2018).

Turkey has a special zoogeographical importance as it forms a continental bridge between Africa, Asia and Europe. It comprises diverse climatic and zoogeographical zones and considerable gradients of elevation, narrow valleys with various subalpine, temperate and Mediterranean habitats. As a result of this geographical and ecological diversity, the country constitutes an important biodiversity hotspot for bees and many other organisms. Moreover, the Turkish insect fauna is an important link in zoogeographical regard between the eastern Mediterranean region and Central Asia. Turkey thus provides a natural route for the spread of species in both an eastwest and a south-north direction. The presentation of these interrelationships using concrete examples is a main purpose of this paper.

So far, no study has dealt specifically with the species of the genus Hylaeus in Turkey. ÖzBEK (1977) studied the Colletidae of Erzurum Province and listed 13 Hylaeus species and one subspecies of this genus occurring there. 
Additionally, ÇALmaşur \& Özbek (1999) and Özbek (2008a, 2008b, 2011) highlighted the importance of bees in the pollination of various cultivated plants, such as alfalfa, sainfoin, sunflower, and various fruit trees and noted certain Hylaeus species visiting these plant species. Alfken (1931) described a single species, Prosopis effasciata, from Mersin. WARnCKE (1972, 1981, 1992) mentioned in his studies on the West Palearctic region more than 40 species which are supposed to occur in Turkey. In WARNCKE (1981) the following eight taxa were described as new (as Prosopis): P. araxana from Iğdır, P. armeniaca from Erzurum, P. nigrita kotschisa from Ankara, P. kurda and P. planula from Hakkâri and $P$. rubosa, $P$. sidensis, and $P$. taurica from Şanlıurfa, Antalya and Mersin provinces respectively. In a later contribution (WARNCKE 1992) he described a further nine new species or subspecies: Prosopis chukar, P. decaocta, P. funerea, $P$. glacialis giresuna and $P$. tephronota from Hakkâri Province, $P$ c cervina from Erzurum, $P$. torquata from Mardin, $P$. trochila from Niğde, and $P$. monedula from Bitlis provinces. In addition, DATHE (2000) described Hylaeus tetris from Isparta. None of these species have been critically revised by examining their types. Moreover, only a few illustrations of them have been published, and an identification key did not exist at all.

\section{Material and Methods}

This study is based on extensive material; over 4000 Hylaeus specimens have been collected throughout the country including Thrace. Although samples from the 1910s and 1950s are also available, most of the specimens have been collected since the 1970s. A major part of the data are provided by H.H. DATHE from his database. It lists the names of over 3400 specimens that he had determined for numerous European collectors. In the last 30 years they have been collected at about 1400 locations. The other part was collected by H. ÖzBEK and his colleagues.

The specimens were mostly caught with insect nets, less frequently using aspirators and Malaise traps. Additionally, if possible, the plants visited by the bees were recorded and vouchers retained for verification. All bee samples and collected plants were properly prepared for long-term preservation in scientific collections. Distribution maps were prepared for new records from Turkey and rare species (known from 1 or 2 provinces). Species are listed alphabetically by subgenus. For some species we did not get specimens for study; data on these were taken from published sources to complete the list.

The allocation of the species to the 81 provinces of the country has proved to be effective in the investigation of Turkish fauna (Ljubomirov \& YILDRIM 2008). They are smaller geographical units than the various ecological regions and provide finer resolution for the evaluation of the record data. In addition, the provinces are grouped into seven regions according to their location and specifics: Marmara, Aegean Sea, Mediterranean Sea and Black Sea Regions, as well as Central, Eastern and SouthEastern Anatolia. The provinces are listed in alphabetical order, their names in bold.

Collection data include collection site, date, altitude in meters above sea level and, if available, decimal latitude/ longitude coordinates, number of male and female specimens, and the collector. Notes refer to distribution and biological data (habitat, flight season, flowers visited, if available), as well as taxonomic remarks. All material examined here, unless otherwise stated, has remained with the European collectors; duplicates are deposited in the SDEI collection. The material collected by H. ÖzBEK and his colleagues is deposited in the Entomology Museum Erzurum (EMET), Turkey.

Full synonymy is given for each species when taxonomic and nomenclatural decisions have to be justified. Otherwise, synonyms are only provided as links to the names used in the relevant literature. Further detailed information about our view of the species, including illustrations, can be found in Dathe (2010, 2014, 2015), Dathe \& Proshchalykin $(2016,2017)$ and Proshchalykin \& DATHe $(2012,2016)$.

Distributions of species are evaluated according to the number of provinces in which they were collected: 1-2 rarely recorded, 3-7 sparsely recorded, 8-17 moderately recorded, 18-above frequently recorded, based on present and previous records.

Our morphological terminology basically follows Michener (2007). However, some terms are specifically in use for the genus Hylaeus. They are applied also in our earlier papers partly in a different way; the main terms and abbreviations used here are defined as follows:

Head

antenna - scape, pedicellus, flagellum with segments $1-10$ (11 in males)

antennal socket - scapus base

mask - facial maculation, consisting of bright (white or yellow) coloration of supraclypeal area, clypeus and paraocular areas; this "complete mask" is characteristic for males; in females the bright spots are mostly limited to the paraocular areas (lateral or side spots). paraocular area - face sides

preoccipital ridge - occiput posteriorly with marginal ledge

\section{Mesosoma}

pronotum - pronotum

mesonotum - scutum, mesoscutum

scutellum - mesoscutellum

axillae - paired sclerites of the mesothorax, appear in dorsal view as "anterolateral corners" of the scutellum, but clearly separated from it by the axillary suture (Michener 2007: 48)

postscutellum - metanotum 
mesopleuron - praeepisternum + mesepisternum anterior edge of mesopleuron - omaulus, actually: angle between anterior and lateral surfaces of mesopleuron; rounded, carinate, lamellate (MicheNER 2007: 48)

legs - pre- (I), meso- (II), meta- (III); e.g. metabasitarsus = basitarsus III

propodeum - basal $(=$ medial $) \sim$, lateral $\sim$, terminal area

\section{Metasoma}

$\mathrm{T} 1, \mathrm{~T} 2, \ldots$ - metasomal terga $1,2, \ldots$

$\mathrm{S} 1, \mathrm{~S} 3, \ldots \mathrm{S} 7, \mathrm{~S} 8$ - metasomal sterna $1,3, \ldots .7,8$

male terminalia - copulatory apparatus: $\mathrm{S} 7+\mathrm{S} 8+$ genital capsule

genital capsule - gonobasis + gonoforcipes + penis valve (parts, dorsally visible)

gonoforceps - gonocoxit + gonostylus (distinguishable only in exceptional cases of Hylaeus males)

\section{Punctation}

Strength and density of the puncturing and their respective combinations are essential characteristics for the differentiation of the species. The gradation follows Dathe (2014: 6).

strength - minute, fine, moderate, strong, coarse, very coarse

density relative to puncture diameter $(\mathrm{d})$ - contiguous (0), subcontiguous $(0.25)$, dense $(0.3-0.7)$, close $(0.7-$ $1.5)$, sparse (2-3), scattered (ca. 3-6).

\section{Identification keys to the Hylaeus species in Turkey}

The best way to get reliable keys is to use a template that has been tested in practical work and is constantly being improved. In this sense, this chapter is an invitation to cooperate. The better this cooperation works, the more new taxonomic, zoogeographical, ecological and bionomic knowledge will emerge, it in turn will lead to changes and make this work obsolete. We would very much welcome this.

\section{Keys to the Subgenera of Hylaeus}

\section{Males}

For the reliable determination of the subgenera (and many species) preparation of the copulatory apparatus (genital capsule plus S7 and S8) is usually unavoidable. When specimens are fresh, it is not difficult to pull the capsule out of the anal cavity using a fine pin (micro pin with hook) and fix it outside.

1 Face entirely black, frons broadly concave and shiny, only the conically swollen scape white; middle basitarsus dilated at base; genital capsule characteristic: short and broad, gonostylus truncate with long, stiff bristles on lateral part of transverse margin nearly as long as gonoforceps H. (Abrupta) cornutus CuRTis

- Face with white or yellow areas ("mask"), or, if exceptionally black, then frons convex and densely punctate; middle basitarsus normal; genital capsule with gonostylus apically rounded or pointed, its bristles shorter than gonoforceps

2 Gonoforceps conspicuously elongated, slender, distal third projects beyond penis valves; apical process of S8 long, hairless, apically with spoon-shaped membrane, usually exposed at anal slit H. (Spatulariella)

- Gonoforceps of normal length and thickness, about as long as penis valves; apical process of S8 not formed as spoon-like membrane, usually concealed in metasoma 3

3 Inner margin of penis valve with raised keel, laterally with flat, rectangular membrane, basal edge of membrane acutely angulate; gonostylus and gonocoxite marked by weak constriction; apical lobes of S7 consist of two pairs of large thin membranes without hairs; apical process of S8 elongated, bilobate, with short bristles; body robust, with coarse punctation H. (Koptogaster)

- Penis valves and gonostylus/gonocoxite variable, but not as above

4 Outline of the two penis valves together cuneiform to spindle-shaped; inner carinae of penis valves in dorsal view are parallel and in close contact up to their apices, or, if separated, then only narrowly so, the ventral structures thus hidden from above 5

- Outline of the two penis valves strongly curved, heart-shaped or circular; inner carinae of penis valves in dorsal view in contact basally but clearly diverging towards the middle, often abruptly bent laterally, inner ventral structures clearly visible from above between penis valves

5 Apical lobes of S7 simple with smooth margins, bristles absent or sparse and at most confined to lateral part of proximal lobe; scape usually slender; labrum and mandible frequently with yellow spots H. (Paraprosopis) 
- Apical lobes of S7 pectinate; scape commonly conically dilated or flattened, but if slender, then sternal callosity or lateral fringe of T1 absent; labrum and mandible black

6 Gonoforceps apically with clearly separated short and pointed appendix, this often pale; gonobase relatively long and conical; S8 apical lobes four-part, the proximal pair with comb teeth, the distal one with smooth rim; scape widened and curved, hollowed out underneath H. (Patagiata)

- Gonoforceps without narrow pale appendix; gonobase short, convex; genital capsule usually smaller and more dainty; apical lobes two-part, with smooth rim or with bristles; scape various

H. (Hylaeus s. str.)

7 Scape conspicuously broadened, shield-like, hollowed out from behind; paraocular area without transverse flat impression; S8 with prolonged basal part and apical process, the latter with short hairs or hairless and hooked downward; the hook usually projecting from a V-shaped incision in S6 H. (Lambdopsis)

- Scape slender or conically enlarged, or, if scutiform, then face with flat transverse impressions on paraocular area and below antennal sockets; S8 more or less rhombiform, with short basal and apical parts, or apical process, if elongated, with hairs and not hooked; S6 rounded or emarginate

8 Penis valves in dorsal view gently bent, between them a pair of acute spines visible, being ventral projections of penis valves; S7 with reduced apical lobes, triangular, with or without sparse fine hairs; S8 strongly reduced, rhombiform, hairless, apical process rarely somewhat elongate and filiform H. (Dentigera)

- Penis valves in dorsal view largely proximate, no spines thus visible from above between penis valves, ventral projections usually short and broad, not spine-like

9 Mesosoma, particularly mesepisterna, strikingly coarsely and strongly punctate; S8 with elongated, curved, slender apical process, its apex with pair of hair tufts; S7 with apical lobes reduced, slender, pointed, with sparse hairs; S6 emarginate in middle H. (Nesoprosopis) pectoralis Förster

- Mesosoma only in relatively few species with noticeably strong punctation, usually finely punctate; S8 rhombic, with short, rounded or truncate, hairless apical process; S7 with reduced apical lobes, compact, with hairs that may be short and sparse; S6 not emarginate

H. (Prosopis)

\section{Females}

1 Clypeus with broad, transverse, saddle-like depression, at all four corners a triangular, pointed projection; face entirely black. H. (Abrupta) cornutus CuRTIS

- Clypeus slightly convex, without projections or teeth; supraclypeal projection absent; face usually with white or yellow mark on paraocular area

2 Mandible tridentate, upper tooth sometimes short and indistinctly visible ......................................................... 3

- Mandible with two teeth or apex bilobate

3 Paraocular spots often elongated, contiguous with inner orbits, or, if face entirely black, then T1 integument (under the punctation) transversely obsoletely reticulated; T1 with lateral fringes, partly indistinct

H. (Dentigera) (brevicornis group)

- Paraocular spots usually rounded, contiguous with clypeal margin, or, if face entirely black, then T1 integument smooth under the punctation; T1 without lateral fringes H. (Lambdopsis) (in part)

4 Vertex swollen, in frontal view surpassing upper ocular margins by ocular width; head in frontal view nearly circular; inner margins of eyes not or only slightly convergent below; genal area broad, or, if head conspicuously rectangular and gena narrow, then thorax red.

H. (Dentigera) (brachycephalus group and H. rubicola SAUNDERs)

- Vertex convex (as usual); head in frontal view rounded or trapezoidal, never rectangular; inner margins of eyes markedly convergent below; genal area narrow; thorax black, with red marks in only a few larger species ....... 5

5 Omaulus carinate or lamellate; malar area at least as long as basal flagellar diameter; thorax and clypeus mostly strongly punctate; propodeal triangle with coarse wrinkles H. (Spatulariella)

- Omaulus rounded or merely angular; malar area shorter than basal flagellar diameter, rarely longer, in which case another character given above does not agree 
6 Head distinctly longer than broad in frontal view; pronotum thickened, dorsolateral angle square-truncate; mesonotum coarsely and strongly wrinkled-punctate; propodeum with horizontal part of basal area very short, distally with sharp edge H. (Koptogaster)

- Head usually shorter, circular or trapezoidal in frontal view; pronotum short, dorsolateral angle rounded or pointed; if mesonotum coarsely punctate, then head always short; propodeum with horizontal part of the basal area of normal length, distally often curved and with or without edge

7 Facial foveae elongated, somewhat surpassing upper ocular margin, converging strongly toward ocelli, terminating closer to ocelli than to compound eye; dorsolateral sides of pronotum angled protruded . H. (Paraprosopis)

- Facial foveae short and straight, barely reaching upper ocular margin and terminating closer to compound eye than to ocelli; dorsolateral sides of pronotum rounded

8 Mesopleura with strikingly coarse and strong but regular pit-like punctures; T1 without lateral fringe, T1 and T2 polished H. (Nesoprosopis) pectoralis FöRsTER

- Mesopleura with fine punctures, or, if coarse and strong, then not regular and metasomal terga densely punctate; $\mathrm{T} 1$ often with lateral fringe, $\mathrm{T} 1$ and $\mathrm{T} 2$ often shagreen

9 Genae laterally on occiput with sharp ridge; pronotum bulging expanded; propodeum surrounded by sharp edge; basal terga smooth and shiny, without punctation, T1 to T4 with white cilia fringes (note the combination of all the attributes) H. (Patagiata)

- Genae laterally rounded; when partly with sharp ridge see Prosopis; pronotum usually not enlarged; propodeum sharply edged or angular; basal terga usually with distinct punctation, T1 to T4 without white end fringes ...... 10

10 Larger species, total length 5-9 $\mathrm{mm}$, usually with short head; facial foveae usually with upper end well separated from eye; propodeum short, at least lateral and posterior surfaces mostly rounded, not delimited by carinae, with fine sculpture and covered with white felt-like hairs; T1 often with lateral fringe of white hairs, but iffringe absent, then metasomal base sometimes red H. (Prosopis)

- Smaller species, total length 3.5-7.0 mm (H. nigritus up to $8 \mathrm{~mm}$ ), with elongated head; facial foveae shorter, upper end close to eye margin; propodeum usually sharp-edged or with carina around posterior surface; metasoma black, usually without lateral fringe on $\mathrm{T} 1$, but if $\mathrm{T} 1$ has fringe, then mesopleura with dense fine punctation and propodeum rounded, with fine sculpture H. (Hylaeus s. str.)

\section{Identification keys to Hylaeus species}

The following identification keys were compiled after examination of material which is as authentic as possible, with regard to Turkey, and in particular on the basis of types. Even for better-known species, specimens of Turkish origin were preferred. As a result, only a limited amount of material from a relatively few sites was available, which certainly does not reflect the variability of the species within the country. The females in particular are often difficult to distinguish, so that attention should always be paid to males flying with them. The best way to prepare reliable keys is to continually test a draft during practical identification work, which will lead to its constant improvement. In this sense, this chapter is an invitation to cooperate. The better this cooperation works, the more new taxonomic, zoogeographical, ecological and bionomic knowledge will emerge, which in turn will lead to changes and make this work obsolete. We would very much welcome this.

\section{Subgenus Dentigera}

H. (Dentigera) acer DATHE, 1980

H. (Dentigera) brevicornis NyLANDER, 1852

H. (Dentigera) chukar (WARNCKE, 1992)

H. (Dentigera) giresunus (WARNCKE, 1992)

H. (Dentigera) glacialis MoRAWITZ, 1872

H. (Dentigera) gredleri FöRSTER, 1871
H. (Dentigera) imparilis FöRsTER, 1871

H. (Dentigera) intermedius Förster, 1871

H. (Dentigera) kahri FöRSTER, 1871

H. (Dentigera) pallidicornis Morawitz, 1876

H. (Dentigera) punctus (Förster, 1871)

H. (Dentigera) rubicola SAUnders, 1850 


\section{Males}

1 Forewing stigma bright, yellowish; head outline in frontal view trapezoidal, almost as high as wide, head length: head width ca. 0.91; scape slim, cone-shaped. pallidicornis MoRAWITz

- $\quad$ Forewing stigma medium to dark brown; head outline in frontal view often elliptic, wider than high; scape slim to strongly thickened, of different shape.

2 Head in frontal view transversely elliptic, markedly wider than long (head length : head width ca. 0.8, Fig. 2f); upper half of supraclypeal area widened almost circularly, about as wide as lower base; scapes slender conical, with white spot at tip. chukar (WARNCKE) Fig. 2

- Head outline diverse, less wide in relation; upper part of the supraclypeal area not expanded, narrower than the base; scapes diverse, often thickened.

3 Head in frontal view long-elliptic; labrum and mandibles white; scape elongated and dilated; terminal area of propodeum smooth and shiny, often brownish. rubicola SAUNDERS

- Head in frontal view trapezoidal; labrum and mandibles black; scape slender or dilated, not elongated; terminal area of propodeum shagreen, rather dull, black.

4 Scape extremely thickened, about as wide as long, mostly extensively pale coloured; S3 with angular hump, this often impressed in in the middle; lateral areas of the propodeum rounded posteriorly.

- Scape slender to distinctly thickened, entirely black or only with small pale spots; S3 with two tubercles or paired calluses; lateral areas of propodeum posteriorly sharp-edged.

5 Mask white, also the clypeus completely white coloured; frons usually with smooth surfaces and less densely punctate; T1 with strong dense punctation, silky shiny.

- Mask yellow, the clypeus often only in the middle with a light stripe; frons up to the supraclypeal area contiguously punctate, without smooth surfaces; basal terga with strong subcontiguous punctation, dull.

punctus Förster

6 Clypeus evenly and gently domed; pronotum with white stripe; mesosoma on underside with scattered white pubescence.

- Clypeus basally flattened, apically strongly bulging; pronotum black; mesosoma on underside with dense and long white hairs.

7 Basal area of propodeum reticulately wrinkled; S3 with strongly curved, often two-part hump.

kahri FÖRSTER

- $\quad$ Basal area of the propodeum striped, with irregular longitudinal ridges; S3 with triangular pointed cusp. acer DATHE

8 The small glossy front surfaces are sparsely punctate up to the supraclypeal area tip; punctation of T1 more shallow and somewhat finer. glacialis MORAWITZ

- The small glossy front surfaces are impunctate in the lower part; punctation of T1 clearer and somewhat coarser (females should be compared for safe differentiation). giresunus (WARNCKE) Fig. 3

9 Scape slender, its lateral edges more or less straight.

- Scape conically enlarged, its lateral edges convex.

10 Outline of the penis valves in dorsal view almost circular; mask variable, but with extensive white areas; scape mostly with apical spots. imparilis FÖRSTER

- Outline of the penis valves in dorsal view long-oval; clypeus often with black anterior margin, mask thus with shield-like outline; scape usually with triangular fleck. intermedius FöRsTER

11 Frons in the middle with scattered punctation, partly striped, but clearly shiny; scape mostly brightly striped along the side. brevicornis NYLANDER

- Frons with dense and strong punctation, only slightly shiny, or matt; scape with wide pale fleck or with small round apical spot. gredleri FÖRSTER 


\section{Females}

1 Wing veins strikingly pale, also stigma and subcosta are yellowish; face usually completely black, occasionally with yellow stripes on the lower orbits. pallidicornis MORAWITZ

- Wing veins medium to dark brown; sides of face pale coloured. 2

2 Head outline in frontal view nearly rectangular; body partly or totally brownish; punctation on metasoma indistinct. rubicola SAUNDERS

- Head outline in frontal view rounded or trapezoidal; body black; terga with distinct punctation. 3

3 Whole clypeus with distinct punctation, evenly curved in profile, only the distal margin impressed. 5

- Basal part of the clypeus shagreen without distinct punctation, its surface depressed, distally with bulge or bent up.

4 Clypeus end margin bent up in the middle and protruding like a lamella; labrum widened in the middle, with long narrow hump. giresunus (WARNCKE) Fig. 3

- Clypeus end margin normal, narrowly impressed; labrum narrow, parallel-sided, in the middle with circular hump. glacialis Morawitz

5 Mask yellow, often also clypeus, supraclypeal area, pronotum band, calli and tegulae with yellow marks; sculpture rough and matt, with contiguous punctation on the mesonotum in front and at the end margin of T1 in the middle. punctus Förster

- Mask white, usually with less bright marks; sculpture mostly with finer punctation, silky shiny. 6

6 Basal area of propodeum striped, with irregular longitudinal ridges; terga depressions broadly horn-coloured translucent; paraorbital area with nearly rectangular spots. acer DATHE

- Basal area of propodeum reticulately wrinkled; terga depressions black or only very narrowly paler; patches of the paraorbital area various.

7 Upper part of supraclypeal area conspicuously broad, almost circular; head outline transverse elliptic, with short clypeus; lateral spots short; T1 only finely and shallowly punctate, smooth and shiny; very small species (total length $4 \mathrm{~mm}$ ). chukar (WARNCKE) Fig. 2

- Supraclypeal area narrow, upper part longer than broad; head mostly trapezoidal; lateral spots larger; T1 distinctly punctate, silky-shiny or dull; usually larger species.

8 Propodeum rounded, side and median areas behind without sharp edges; lateral areas on the horizontal part with the same fine sculpture as on the terminal area.

- Terminal area of the propodeum with sharp surrounding edge or lateral areas at least with distinct step; if edge indistinct, the lateral areas on the horizontal part have a coarser sculpture than on the terminal area

9 Lateral spots short and narrow, sometimes absent; clypeus and usually also pronotum black; frons above the supraclypeal area with sparse punctation, shiny. brevicornis NYLANDER

- $\quad$ Lateral spots long, linear on the orbits, or filling the facial sides; clypeus and pronotum often broadly bright; frons above the supraclypeal area with close punctation, silky or dull.

10 Head slightly longer; colouration of head and thorax broadly pale, lateral spots often filling the paraocular areas, and clypeus spotted; front margin of clypeus only narrowly impressed. kahri FöRSTER

- Head broader; lateral spots often elongated rectangularly to the orbits; clypeus not spotted, front margin with conspicuously broad impression. intermedius FöRSTER

11 Lateral spots long linear, adjacent to the orbits; frons above the supraclypeal area with dense punctation, dull; mesonotum coarsely sculptured, shiny. gredleri FöRSTER

- Lateral spots large, fill the paraorbital field, or are at least expanded inward; frons above the supraclypeal area with more scattered punctation, shiny; mesonotum in front finely sculptured, matt. imparilis FÖRSTER 
Subgenus Hylaeus s. str.

H. (Hylaeus) angustatus (SCHENCK, 1861)

H. (Hylaeus) araxanus (WARNCKE, 1981)

H. (Hylaeus) communis NylANDER, 1852

H. (Hylaeus) crispulus DATHE, 1980

H. (Hylaeus) deceptorius (BEnOIst, 1959)

H. (Hylaeus) dolichocephalus Morawitz, 1876

H. (Hylaeus) funereus (WARNCKE, 1992)

H. (Hylaeus) gracilicornis (MoRAwITz, 1867)

H. (Hylaeus) implicatus DATHE, 1980

H. (Hylaeus) jantaris DATHE, 1980

H. (Hylaeus) kotschisus (WARNCKE, 1981)

H. (Hylaeus) kurdus (WARNCKE, 1981)

H. (Hylaeus) leptocephalus (MorAwitz, 1871)
H. (Hylaeus) monedulus (WARNCKE, 1992)

H. (Hylaeus) moricei (FrIESE, 1898)

H. (Hylaeus) nigritus (FABRICIUs, 1798)

H. (Hylaeus) orientalicus (WARNCKE, 1981)

H. (Hylaeus) paulus Bridwell, 1919

H. (Hylaeus) scutellaris Morawitz, 1874

H. (Hylaeus) sidensis (WARNCKE, 1981)

H. (Hylaeus) tardus (WARNCKE, 1981)

H. (Hylaeus) torquatus (WARNCKE, 1992)

H. (Hylaeus) trifidus (AlfKen, 1936)

H. (Hylaeus) trisignatus Morawitz, 1876

H. (Hylaeus) trochilus (WARNCKE, 1992)

H. (Hylaeus) tyrolensis FöRSTER, 1871

\section{Males}

1 Mask yellow

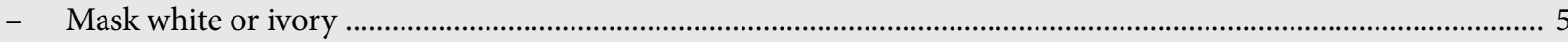

2 Scutellum yellow-marked, femora almost all yellow, mesopleura with very coarse contiguous punctation scutellaris MORAwITZ

- Scutellum black, femora broadly black, mesopleura with less coarse punctation 3

3 Very small species, total length $4-5 \mathrm{~mm}$; propodeum at least with the lateral areas rounded; mask light yellow, dull ..... paulus BRIDWELL

- Larger species, total length >5 mm; propodeum sharply rimmed around margin; mask strongly yellow, silky shiny

4 Scape longer and wider, markedly wider than the flagellum at its widest site; apical lobus of S8 only spatula-like dilated, without bristles; T1 polished, with fine scattered punctation communis NYLANDER

- Scape shorter and slimmer, only slightly wider than the flagellum; apical lobus of S8 with paired roof-like parts with fine bristles at margin; T1 polished, with moderate sparse punctation deceptorius (BENOIST)

5 Paraocular area deeply gutter-like impressed, the furrows converging downwards; clypeus shorter than supraclypeal area; mask characteristic: with white clypeus and long side stripes at the orbits, otherwise nearly completely black (Fig. 7a); scape enlarged, about as wide as long, outer half white; very small species orientalicus (WARNCKE)

- $\quad$ Larger species with different formation of the face

6 Scape completely black, at most sometimes with a minute white apical spot

- Scape with broad pale marking

7 Face below the antenna bases with strong arched transverse impression, the clypeus bulging up 8

- Face evenly bulged, the clypeus included in this profile

8 Upper part of the mask button-like, lateral spots at the top separated from the orbits by a narrow black surface; scape slender, without apical impression

- Mask not button-like constricted above, the lateral spots tapering towards antennal sockets above; scape expanded, apical with flat impression kurdus (WARNCKE) Fig. 5

9 Clypeus only flat arched; mask longitudinally striped, matt; mesopleura in front edged angustatus (SCHENCK)

- Clypeus strongly domed; mask smooth and glossy; mesopleura in front rounded crispulus DATHE

10 Larger (total length $>5 \mathrm{~mm}$ ) often robust species, propodeum coarsely net wrinkled but rounded; with transverse callosity or hump on S3

- Smaller and more dainty species; propodeum with terminal area often sharply rimmed, and/or sterna without callosity or hump 
11 Clypeus evenly convex, integument silk-matt, punctation close, appearing as transverse wrinkle stripes; lateral white spots end dorsolaterally separated from the orbits by a black pit; scape slim jantaris DATHE

- Clypeus with shallow longitudinal impression, integument smooth and shiny, punctation scattered, indistinct; lateral white spots dorsally adjacent to the orbits; scape expanded

12 Supraclypeal area trapezoidal, below 0.8 times as wide as the distance to the orbits; scape expanded, about 1.9 times longer than wide, black nigritus (FABRICIUS, 1798)

- Supraclypeal area rectangular, only about half as wide as the distance to the orbits; scape somewhat less expanded, 1.7 times longer than wide, with a minute white spot at tip kotschisus (WARNCKE, 1981)

13 Frons broadly impressed, the surface with a striking special formation 14

- Frons not broadly impressed, punctate, without special formation

14 Frons impression with wrinkled stripes and covered with short silvery hairs, matt moricei (FRIESE)

- Frons impression flat, built as semicircle enclosing the anterior ocellus, longitudinally streaked, without punctation; scape slim, often with elongate triangular apical spot araxanus (WARNCKE)

15 Mesopleura with strong close punctation, intervals highly glossy 16

- Mesopleura with denser punctation, intervals silky shiny or matt 17

16 Mask glossy; lateral spots end above at orbits; clypeus below only with narrow black seam; scape narrow, blackish brown lightened; legs predominantly white, tibiae only with dark spot tardus (WARNCKE)

- Mask silky shiny; lateral spots end above separated from orbits; clypeus below with black margin; scape slightly expanded, black; tibiae II and III apically with black ring spot monedulus (WARNCKE) Fig. 6

17 T1 shiny, with minute or fine sparse to scattered punctation 18

- $\quad$ T1 matt, with strong dense to subcontiguous punctation 19

18 Mask yellowish-white, supraclypeal area black; above the lateral spots only with narrow shiny line; basal area of the propodeum delimited by marginal ridge; T1 smooth and glossy, with minute scattered punctation

gracilicornis (MORAWITZ)

- Mask pure white, supraclypeal area white up to scape bases; next to and above the lateral spots at the eye margin with broad shiny furrow; basal area of the propodeum without border ridges; T1 finely grooved on the end margin, with fine sparse punctation funereus (WARNCKE) Fig. 4

19 Gonoforcipes apically rounded, not expanded, bristles normal; frons only at the lower end next to the supraclypeal area-tip without punctation; pronotum white spotted tyrolensis FÖRSTER

- Gonoforcipes apically expanded, with long bristles; frons in the middle with shiny surface without punctation; pronotum black

20 Gonoforcipes apically strongly expanded to circular discs; with long feathered bristles; frons with silky shiny areas in the middle; mandibles black; head and mesosoma normal short haired sidensis (WARNCKE) Fig. 8

- Gonoforcipes apically rounded; with conspicuously long simple bristles; frons with larger shiny areas in the middle; mandibles white; head and mesosoma densely short-haired torquatus (WARNCKE) Fig. 9

21 Tergal depression apically with white felt fringes or band; face clearly extended; malar space long, about as long as flagellum diameter dolichocephalus MorAwITZ

- Depression of the terga apically without felt fringes; face shorter; malar space narrower 22

22 Face below the antenna bases with strong arched transverse impression, the clypeus bulged up trochilus (WARNCKE) Fig. 10

- Face evenly curved in profile, the clypeus included into this profile

23 Scape short, not expanded, apical with small triangular spot; paraocular area next to the antenna bases with shiny black surface at the orbits reducing the lateral spots to a narrow tip trifidus (AlfKeN)

- Scape of normal length, mostly expanded and amply white; paraocular area without lateral shiny black areas, lateral patches sprawling 
24 Mesopleura with conspicuously coarse punctation, distinctly coarser than on the mesonotum; T1 with moderate close punctation leptocephalus (Morawitz)

- Mesopleura with moderate to strong punctation, about as strong as the mesonotum; T1 with fine sparse punctation

25 Head wider than long; mask shiny, hardly punctate; lateral spots extending over the antennal sockets into a smooth area; mesosoma densely hairy; T1 almost impunctate, smooth and shiny trisignatus MORAWITz

- Head elongate trapezoidal; mask shagreen and shallowly punctate, silky shiny; lateral spots truncate above; mesosoma only slightly hairy; T1 with fine sparse punctation implicatus DATHE

\section{Females}

1 Paraocular areas with yellow lateral spots

- Paraocular areas with white or ivory lateral spots or without spots

2 Scutellum yellow-spotted, femora almost all yellow; mesopleura with very coarse contiguous punctation; mesopleura frontally sharp-edged scutellaris MORAWITZ

- Scutellum black, at least femora broadly black; mesopleura with moderate to strong dense punctation; mesopleura in front angular communis NYLANDER

3 T1 smooth, with very scattered fine punctation; pronotum mostly black deceptorius (BENOIST)

- T1 smooth, with moderate sparse punctation; pronotum yellow 5

4 Face with bright spots 20

- Face completely black

5 Head extremely extended, strongly narrowed downwards; T1-T4 with felt fringes; malar space as long as wide; metabasitarses white dolichocephalus Morawitz

- Head outline different, often about as long as wide; terga without dense felt fringes; malar space short or narrow; metabasitarses black or white

6 Clypeus with broad longitudinal impression; lateral spots fill the paraocular area up to the scape bases; integument smooth and shiny

- Clypeus without broad longitudinal impression, evenly arched; lateral spots and integument sculpture various ..

7 Mesonotum with moderate sparse punctation; depressions of T2 and following terga only slightly pale horncoloured; larger species, total length $7-8 \mathrm{~mm}$ nigritus (FABRICIUS)

- Mesonotum with somewhat denser (close) punctation; depressions of T2 and following terga brightly horncoloured; smaller species, total length 6-7 mm kotschisus (WARNCKE)

8 Propodeum with sharp ledges around, the ridge is clearly visible even in rough sculptures behind the basal area and the lateral areas as a cross-ledge

- Propodeum rounded or edged, behind the lateral areas no distinct ledge visible

9 Mesopleura with coarse close punctation, pits significantly coarser than on the mesonotum

- Mesopleura with moderate or strong punctation, punctation of the mesonotum resembles that of the mesopleura

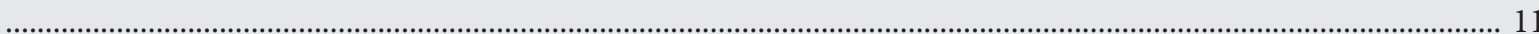

10 Somewhat larger species (total length 5-6 $\mathrm{mm}$ ) with long trapezoidal head contour; metabasitarses black leptocephalus Morawitz

- Small species (total length $4.5 \mathrm{~mm}$ ) with transverse rounded trapezoidal outline of the head; metabasitarses white tardus (WARNCKE)

11 Head distinctly longer than broad; lateral spots filling the paraocular area, reaching above beyond the scape base; pronotum with white band or long stripes; metabasitarses black implicatus DATHE 
- Head wider than long; lateral spots reduced, upper part if filling truncated, or as spots; pronotum with short white stripes; metabasitarses white

12 Basal area of propodeum basally with a row of short longitudinal ridges only; mesosoma compact; terminal part of T1 with fine sparse punctation; slightly larger species, total length $5 \mathrm{~mm}$.

monedulus (WARNCKE) Fig. 6

- Basal area of propodeum irregularly reticulated wrinkly; mesosoma slender; terminal part of T1 with minute scattered punctation; very small species of $4 \mathrm{~mm}$ total length

13 Clypeus usually with white spot, sometimes also supraclypeal area spotted; mask amply white

trifidus (ALFKEN)

- Clypeus and supraclypeal area black; mask often reduced to two spots araXanus (WARNCKE)

14 Propodeum rounded, with fine shagreen sculpture, basal area only at the base with short, tapering ridges ...... 15

- Propodeum rounded or edged, with extended wrinkled sculpture, at least basal area with net wrinkles 16

15 Head transversely oval; clypeus excised in front, with indistinctly outlined bell-shaped central spot; lateral spots are attached to the sutures of supraclypeal area and clypeus; T1 with very scattered minute punctation

trisignatus MORAWITz

- Head long trapezoidal; clypeus only shallowly excised, black; lateral spots very small, being in the lower part of the paraocular area; $\mathrm{T} 1$ with sparse fine punctation kurdus (WARNCKE) Fig. 5

16 T1 with moderate dense punctation, margin in the middle narrow pointless; pronotum and metabasitarses white spotted crispulus DATHE

- $\quad$ T1 with fine or minute sparse or scattered punctation, margin in the middle wide pointless; pronotum and metabasitarses black

17 Terminal area of the propodeum separated from basal area and lateral areas by irregular fine ridges; head outline longer

- Terminal area of the propodeum not delimited by fine ridges all around; head outline short, roundish 19

18 Mesopleura in front angular and often slightly bent up, so that the anterior surface appears flat; T1 with fine sparse punctation angustatus (SCHENCK)

- Mesopleura in front rounded, anterior surface convex; T1 with minute scattered punctation ...... moricei (FRIESE)

19 Medial furrow of the terminal area flat and relatively broad, tapering above into ridges bordering the basal area laterally; lateral spots of the face below, small, sometimes absent gracilicornis (MORAWITZ)

- Medial furrow of the terminal area deepened, also impressed at the top, but not tapering into ridges, border of the basal area laterally indicated only by altered shagreen; lateral spots of the face as short lines, sometimes absent..

paulus BRIDWELL

20 Propodeum rounded and finely shagreen, silky shiny; only base of basal area with merging meshes and longitudinal ridges; completely black except underside of flagella, tegulae and tibial bases funereus (WARNCKE) Fig. 4

- $\quad$ Propodeum edged or rounded, with rough sculpture and shiny meshes; base of basal area mostly net wrinkled; white coloration more extended or even completely black

$21 \mathrm{~T} 1$ and mesonotum with the same strong dense punctation, dull; flagella very short, underside yellow; pale coloration otherwise only at the edge of the calli, at the tibial bases, tegulae with spot sidensis (WARNCKE) Fig. 8

- $\quad$ T1 with substantially weaker punctation than mesonotum, glossy; flagellae longer; pale colouring often more extended

22 Larger species, total length 5-6 mm; T1 smooth and glossy, with moderate sparse punctation; metabasitarses white spotted; terga with broad horn-coloured brightened depressions jantaris DATHE

- Smaller species, total length 4-5 mm; T1 finely shagreen, glossy, with minute to fine scattered punctation; metabasitarses black; terga apical with at most narrow and indistinctly brightened depressions

23 Frons strongly punctate to the tip of the supraclypeal area; T1 very fine striped, punctation obsolete and very scattered orientalicus (WARNCKE) Fig. 7 
- Punctation of the frons leaves out small mostly shiny areas next to the tip of the supraclypeal area; T1 finely striped, but a fine punctation is perceptible

24 Head contour long trapezoidal; supraclypeal area and clypeus base longitudinally striated trochilus (WARNCKE) Fig. 10

- Head contour rounded, wider than long; supraclypeal area und clypeus base with coarse punctation

25 Frons next to the tip of the supraclypeal area with small pointless areas, these smooth and shiny; mesopleura with coarse dense punctation, pits larger than on the mesonotum; T1 with fine sparse punctation

torquatus (WARNCKE) Fig. 9

- Small front surfaces next to the tip of the supraclypeal area shagreen, matt; mesopleura with strong sparse punctation, pits in size similar to those on the mesonotum, but punctation much more dispersed; T1 with minute scattered punctation tyrolensis FöRSTER

\section{Subgenus Koptogaster}

H. (Koptogaster) bifasciatus (JuRINE, 1807)

H. (Koptogaster) punctulatissimus SMITH, 1842

H. (Koptogaster) tetris DATHE, 2000

\section{Males}

1 Scapes black; mesonotum surface even, with dense punctation; T1 with dense or subcontiguous moderate punctation, intervals silky shiny or matt; axillae black or white

- Scapes with white stripes; mesonotum surface wrinkled, with very coarse punctation; T1 with close strong punctation, intervals smooth and glossy, axillae white bifasciatus (JURINE)

2 Scutellum and axillae black; punctation of T1 dense, intervals shiny punctulatissimus SMiTH

- Scutellum and axillae laterally with white basal spots each (four spots in all); T1 with contiguous to subcontiguous punctation, surface appearing matt tetris DATHE

\section{Females}

1 Metasoma all black; mesonotum plane, with dense punctation; intervals silky shiny only

- Metasoma base (T1 and T2) red; mesonotum surface wrinkled, with very coarse punctation; intervals smooth and shiny bifasciatus (JURINE)

2 Scutellum and axillae black; pronotum with lateral white spots punctulatissimus SMiTH

- Scutellum and axillae laterally with white basal spots each (four spots in all); pronotum with large white flecks.. tetris Dathe

\section{Subgenus Lambdopsis}

H. (Lambdopsis) crassanus (WARNCKE, 1972)

H. (Lambdopsis) scutellatus (SPINOLA, 1838)

H. (Lambdopsis) dilatatus (KIRBY, 1802)

H. (Lambdopsis) euryscapus Förster, 1871

H. (Lambdopsis) tephronotus (WARNCKE, 1992) 


\section{Males}

The male of H. tephronotus (WARNCKE) is not known.

1 Front edge of the mesopleura sharply extended and curved up; mesosoma conspicuously smooth and shiny ....... crassanus (WARNCKE)

- Mesopleura rounded at front; mesosoma silky shiny or matt

2 Mandibles black; sterna flat, without elevations euryscapus FöRSTER

- Mandibles broadly marked white; sterna with calluses or humps 3

3 Scape strongly widened, almost twice as wide as long; only few white spots on the mesosoma: small stripes on the pronotum, the calli and tegulae; S3 and S4 with flat transverse calluses dilatatus (KIRBY)

- Scape less expanded, only slightly wider than long; mesosoma with richly light-yellow marks: pronotum band, frontolateral mesonotum corners, calli, tegulae, axillae, scutellum and postscutellum; S3 to S5 with humps

scutellatus (SPINOLA)

\section{Females}

1 Front edge of the mesopleura sharply extended and curved up; mesosoma conspicuously smooth and shiny crassanus (WARNCKE)

- Mesopleura rounded at front; mesosoma silky shiny or matt 2

2 T1 glossy, polished at least on the domed base, without punctation; mesosoma and clypeus black 3

- T1 matt, throughout with close strong punctation; mesosoma mostly with light yellow spots: pronotum band, calli, tegulae, axillae, scutellum, clypeus; the latter sometimes completely yellow scutellatus (SPINOLA)

3 Outline of head rounded-trapezoidal; face with white spots beside the clypeus base; pronotum drawn white; front of clypeus without long bristles; larger species of approx. $6 \mathrm{~mm}$ total length.

- Outline of head nearly circular, slightly wider than long; face and pronotum black; clypeus in front with a row of strikingly long bristles; dainty species of approx. $4.5 \mathrm{~mm}$ total length tephronotus (WARNCKE) Fig. 11

4 T1 and T2 with the same fine punctation, slightly denser on T2; integument less shiny dilatatus (KIRBY)

- T1 with distinctly coarser punctation than T2, punctation on T2 denser; integument as a whole strongly glossy .. euryscapus FöRSTER

\section{Subgenus Paraprosopis}

H. (Paraprosopis) clypearis (SCHENCK, 1853)

H. (Paraprosopis) decaocto (WARNCKe, 1992)

H. (Paraprosopis) lineolatus (SCHENCK, 1861)

H. (Paraprosopis) pictipes NylandeR, 1852
H. (Paraprosopis) sinuatus (SCHENCK, 1853)

H. (Paraprosopis) soror (PÉREZ, 1903)

H. (Paraprosopis) styriacus FöRster, 1871

H. (Paraprosopis) taeniolatus FöRSTER, 1871

\section{Males}

1 Clypeus wholly or partly black, in particular apical; anterolateral pronotum sides acute; lateral fringes absent on T1

- Clypeus entirely white or yellow, occasionally darkly lined at front margin; anterolateral pronotum sides edged or rounded; lateral fringes present on $\mathrm{T} 1$, but often indistinct

2 Clypeus entirely black; face with two lateral, high set yellow-white stripes at the orbits; S3 and S4 with transverse bulge; gonoforcipes apically widened and flattened, considerably longer than the penis valves lineolatus (SCHENCK) 
- Clypeus with only lower half black, mask otherwise complete; gonoforcipes conventional; metasoma below flat; penis valves relatively longer clypearis (SCHENCK)

3 Head outline transverse trapezoidal, with rich yellow mask; lateral spots long and broad, covering most of the frons and reaching the vertex decaocto (WARNCKE) Fig. 12

- Head outline rounded or elongated, with mask white or light yellow; lateral spots smaller, do not reach vertex ...... 4

4 Gonoforceps apically narrowly pointed; T1 polished, moderately punctate pictipes Nylander

- Gonoforceps apically rounded or with broad tip; T1 shagreen or densely punctate 5

5 T1 densely shagreen and punctate; free part of gonoforcipes significantly shorter than their fused base styriacus FÖRSTER

- T1 smooth or only indistinctly grooved, deeply punctate; free part of gonoforcipes clearly longer than their fused base taeniolatus FörSTER

6 Scape slender, almost three times as long as wide; legs entirely light yellow from femur tip 6

- Scape widened, about twice as long as broad; tibiae and tarsi with black spots 7

7 Lateral spots above antennal sockets expanding inward; scape with white edge strip; head in outline wider than long sinuatus (SCHENCK)

- Lateral spots above antennal sockets straight, not expanding inward; scape predominantly white; head in outline about as long as wide soror (PÉREZ)

\section{Females}

1 Scutellum, axillae and postscutellum yellow; mask yellow: paraocular areas completely, clypeus and supraclypeal area with round spot; scape with yellow apical spot decaocto (WARNCKE) Fig. 12

- Mesosoma upper side black; scape black; less richly coloured, pale areas light yellow or white 2

2 Head long, trapezoidal; facial lateral spots linear, adjoining orbits along their length lineolatus (SCHENCK)

- Head transverse or roundish; lateral spots punctiform to triangular, sometimes filling the paraocular areas ....... 3

3 Pronotum corners pointed; mandibles three-toothed; T1 with moderately close punctation, without lateral fringes; paraocular areas completely yellow clypearis (SCHENCK)

- Pronotum corners rounded; mandibles bidentate; T1 shagreen or with shallow scattered punctation; narrow side fringes present; lateral spots various

4 Terga densely shagreen, punctation nearly indistinguishable in the background sculpture; lateral spots white, large and triangular; clypeus with small yellow spot styriacus FöRSTER

- Terga not or only shallowly shagreen; punctation distinct, also on T2; lateral spots frequently reduced 5

5 Mesopleura with shallow fine sparse punctation on fine shagreen-coated surface; silky shiny

- Mesopleura with moderately close punctation; glossy

6 Richly marked with light yellow: lateral spots usually filling the paraocular areas, clypeus often with spot, pronotum band present; $\mathrm{T} 1$ with fine sparse punctation taeniolatus Förster

- Less richly pale-marked, lateral spots often abbreviated above and below, pronotum with two short stripes or black; T1 with fine close punctation pictipes Nylander

7 Head shorter, wider than long; lateral spots truncated at top and bottom; clypeus without spot; T1 with fine sparse punctation sinuatus (SCHENCK)

- Head longer, hardly wider than long; lateral spots filling the paraocular areas; clypeus with spot; T1 with minute scattered punctation soror (PÉREZ) 


\section{Subgenus Patagiata}

H. (Patagiata) cervinus (WARNCKe, 1992)

H. (Patagiata) difformis (Eversmann, 1852)

1 Males

- Females 3

2 Occiput posteriorly with sharp ridge; upper suture line of clypeus almost as wide as its distance to the orbits; gonoforcipes apically with pale appendix difformis (EVERSMANN)

- Occiput posteriorly without ridge, rounded; upper suture line of clypeus wider than its distance to orbits; gonoforcipes apically narrowly constricted, appendix of same colour cervinus (WARNCKE)

3 Occiput surrounded by sharp ridge; T1 without punctation, polished and very shiny; lateral spots limited to the area below the scape bases difformis (EVERSMANN)

- Occiput posteriorly without ridge, rounded; T1 polished, but with close strong punctation; lateral spots extended along orbits to beyond scape bases cervinus (WARNCKE)

\section{Subgenus Prosopis}

H. (Prosopis) confusus Nylander, 1852

H. (Prosopis) damascenus (Magretti, 1890)

H. (Prosopis) duckei (Alfken, 1905)

H. (Prosopis) excelsus (Alfken, 1935)

H. (Prosopis) gibbus SAUnders, 1850

H. (Prosopis) incongruus Förster, 1871

H. (Prosopis) maculatus (Alfken, 1904)

H. (Prosopis) meridionalis FöRsteR, 1871
H. (Prosopis) pictus (Smith, 1853)

H. (Prosopis) rubosus (Warncke, 1981)

H. (Prosopis) rugicollis Morawitz, 1874

H. (Prosopis) signatus (PANzer, 1798)

H. (Prosopis) stellatus (WARNCKE, 1992)

H. (Prosopis) trinotatus (PÉREz, 1896)

H. (Prosopis) variegatus (FABRICIUs, 1798)

H. (Prosopis) variolaris MoRAwitz, 1876

\section{Males}

1 Scape slim, in frontal view twice to three times as long as wide, often completely black ..................................... 2

- Scape expanded, triangular or shield-like, with white or yellow marks .............................................................. 10

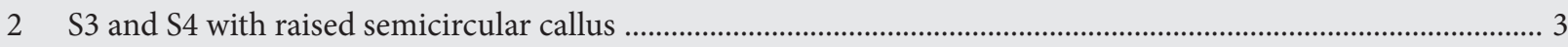

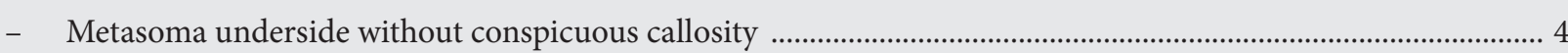

3 T1 with strong dense to subcontiguous punctation on shagreen surface, weakly shiny; mask white, silky shiny; scape with narrow longitudinal yellow stripe signatus (PANZER)

- $\quad$ T1 with only minute very scattered punctation on glossy surface; mask yellow, matt; scape black trinotatus (PÉREZ)

4 Scape all black, about twice as long as wide; mesopleura glossy; T1 with dense strong punctation 5

- Scape with white or yellow marks, only exceptionally all black, about three times as long as wide; mesopleura weakly shiny, T1 with fine to moderate scattered punctation (gibbus group)

5 Punctation of mesopleura distinctly stronger than on the mesonotum; occiput rounded in the middle duckei (ALFKEN)

- Punctation of mesopleura about as on the mesonotum; occiput sharp-edged in the middle stellatus (WARNCKE) Fig. 14

6 Mask white; scutellum and postscutellum always black, mostly also labrum and pronotum; lateral spots abbreviated above 
- Mask yellow; scutellum and postscutellum often yellow, as well as labrum and pronotum; lateral spots extend far beyond the antennal sockets at the top

7 Metasoma dorsally very finely shagreen, silky shiny; with ciliary bands on the depressions pictus SMITH

- Metasoma dorsally smooth, very shiny; without ciliary bands at the end margins

damascenus (MAgRetTi)

8 Frons with supraantennal impunctate areas large, distinctly longer and wider than antennal sockets; mask yellowwhite; lateral spots do not reach beyond the upper edge of the antennal sockets; preoccipital ridge present, but sometimes indistinct incongruus Förster

- Frons with supraantennal impunctate areas smaller, approximately the size of the antennal sockets; preoccipital ridge various, present or absent

9 Head shorter, without preoccipital ridge, rounded; T1 only with very scattered shallow punctation

Head longer, with distinct sharp preoccipital ridge; $T 1$ with distinct fine punctation confusus NYLANDER

10 Face sides with strong oblique impressions converging downward; supraclypeal area wide gibbus SAUNDERS scape shield-like expanded

Face sides without converging impressions; supraclypeal area normal, its ventral part impressed; scape widened, distinctly longer than broad (variegatus group)

11 Mesopleura coarsely wrinkled with pit-like subcontiguous punctation; mesonotum with coarse punctation, intervals glossy; mask and the rich pale flecks on the mesosoma yellow; metasomal terga reddish yellow, T3-T6 with dark end margins; T1 polished with strong sparse punctation excelsus (ALFKEN)

- Mesopleura even, smooth, with dense punctation; mesonotum much more finely and densely punctate, little shiny; mask and flecks of mesosoma white; metasoma black or only the basal terga reddish-yellow; T1 with very dense to subcontiguous punctation

12 Metasomal base reddish-yellow; axillae white, scutellum with two white spots; hind tibia entirely white, only with indistinct brown spot maculatus (ALFKEN)

- Metasoma usually all black, occasionally (locally) with T1 yellow-red; axillae white, but scutellum black without spots; hind tibia with broad black apical ring

13 Face impressed only in the middle, especially the supraclypeal area; paraocular areas finely striped and with close punctation up to the clypeus margins; scape at most half white, flagellum dark brown to black

variegatus (FABRICIUS)

- Face strongly impressed throughout the middle face, including the paraocular areas and the clypeus base; paraocular areas smooth and only with scattered punctation; scape white except for narrow stripe on the edge; flagellum red, rarely brown meridionalis FöRSTER

14 Integument rust-brown throughout; with rich ivory-white coloration: labrum and mandibles, scapes completely, pronotum band, spots on mesosoma above and behind calli, axillae, scutellum with two spots, postscutellum, legs from femur tip rubosus (WARNCKE) Fig. 13

- Integument predominantly black; metasoma base black or red

15 Scape much wider than long; metasoma base rust-red, the terga with dense strong punctation, T2 and following terga with transparent depressions; axillae and two spots on the posterior scutellum margin white, the metanotum also may be white-striped rugicollis MORAWITZ

- Scape scarcely wider than long; metasoma base black, terga with sparse moderate punctation, their depressions only slightly pale; axillae, scutellum and metanotum completely black variolaris MORAWITZ 


\section{Females}

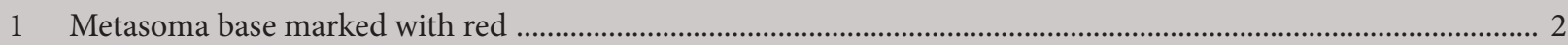

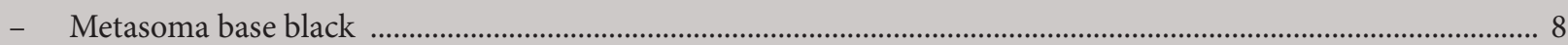

2 Face white- or yellow-spotted; axillae white, only exceptionally black ............................................................ 3

- Face black, sometimes lower parts brownish; axillae white or black ........................................ variolaris Mor MWITZ

3 Integument entirely rust-brown; with rich ivory-white coloration on clypeus, pronotum, calli, axillae, scutellum, postscutellum, legs distally from femur tip rubosus (WARNCKE) Fig. 13

- Integument of mesosoma mostly black, metasoma base black or rust-red

4 Mesopleura coarsely wrinkled with very coarse subcontiguous punctation; anterior edge of mesopleura bent up, sharp-edged; metanotum white

- Mesopleura evenly smooth, densely punctate; anterior edge of mesopleura rounded; metanotum black 6

5 Bright coloration ivory white; T1 and T2 rust-red; scape black with tip spot ..... rugicollis MORAWITZ

- Bright coloration lemon yellow; T1-T3 reddish yellow; scape yellow with dark side stripe excelsus (AlfKeN)

6 Scutellum black

- Scutellum with paired white spot maculatus (ALFKEN)

7 Mesopleura smooth, with subcontiguous strong punctation, interspaces silky shiny; clypeus usually black variegatus (FABRICIUS)

- Mesopleura wrinkled, matt, punctation coarse, interspaces often not recognizable; altogether more richly pale coloured, white marks more extensive, clypeus usually with white spot or median stripe ...... meridionalis FöRSTER

8 Front edge of mesopleura below angular and slightly bent up; basal area of the propodeum with sharp net wrinkles signatus (PANZER)

- Mesopleura rounded in front, not bent up; basal area of the propodeum with sculpture tending to longitudinal ridges 9

9 Clypeus black, sometimes with pale spot, silky shiny 11

- Clypeus yellow or extensively rust-brown, matt 10

10 Clypeus almost entirely light yellow, at most laterally narrowly brown; genae narrow; scutellum and metanotum black; T1 polished without punctation trinotatus (PÉREZ)

- Clypeus rust-brown, middle with wide, long yellow stripe; genae nearly half as long as wide; scutellum and metanotum yellow spotted; T1 minutely shagreen with fine punctation damascenus (MAGRETTI)

11 T1 surface distinctly shagreen, with dense fine punctation, silky shining; head wider than long 12

- $\quad$ T1 surface only very fine shagreen, with scattered minute punctation, shiny; head as wide as long or longer (gibbus group)

12 Punctation of mesopleura irregular, distinctly stronger than on the mesonotum; occiput edge rounded in the middle duckei (ALFKEN)

- $\quad$ Punctation of mesopleura about as on the mesonotum, regular; occiput in the middle sharp-edged stellatus (WARNCKE)

13 T1 with distinct dense punctation; terga apically becoming paler, with dense ciliary bands; mostly richly yellowmarked are: clypeus, scutellum, pronotum and calli (there are also darker forms with black scutellum)

pictus SMITH

- $\quad$ T1 with fine scattered punctation, or impunctate; terga at the end brown, ciliary banding indistinct; clypeus usually black; pronotum with two bright stripes or black; scutellum black .....

14 Supraantennal impunctate areas larger, about as long or longer than antennal socket; clypeus apically with shallow but distinct punctation incongruus Förster

- Supraantennal impunctate areas smaller, not longer than antennal socket; clypeus apically with scattered shallow punctation 
15 Head longer, occiput with distinct sharp marginal ledge; T1 finely shagreen, with scattered but distinct fine punctation gibbus SAUNDERS

- Head shorter, occiput rounded, without sharp marginal ledge; T1 only weakly shagreen, with minute very scattered shallow punctation confusus NYLANDER

\section{Subgenus Spatulariella}

H. (Spatulariella) adspersus (ALFKEN, 1935)

H. (Spatulariella) alpinus (MorAwitz, 1867)

H. (Spatulariella) armeniacus (WARNCKE, 1981)

H. (Spatulariella) cypricola (WARNCKE, 1972)

H. (Spatulariella) hyalinatus SмIтн, 1842

H. (Spatulariella) iranicus DATHE, 1980
H. (Spatulariella) irritans DATHE, 1980

H. (Spatulariella) longimacula (ALFKen, 1936)

H. (Spatulariella) planulus (WARNCKE, 1981)

H. (Spatulariella) punctatus (BRullé, 1832)

H. (Spatulariella) sulphuripes (GRIBOdo, 1894)

H. (Spatulariella) tauricus (WARNCKE, 1981)

\section{Males}

$1 \quad$ T1 red; mask sulphur yellow, complete; scape predominantly yellow; legs from femoral tip entirely yellow; S8 Fig. $1 \mathrm{~g}$ sulphuripes (GRIBODO)

- $\quad$ T1 black; mask ivory white, light yellow or reduced; scape predominantly black; legs black with white flecks

2 Mask reduced: supraclypeal area, paraocular areas and clypeus all or mostly black; scape short, slimmer than the flagellum

- Mask completely pale, some parts at most slightly blackened; scape various

3 Lateral spots fill the paraocular area up to antennal sockets; only supraclypeal area all black; S8 Fig. $1 \mathrm{j}$ longimacula (ALFKeN)

- Lateral spots very small or absent

4 Clypeus with rectangular white spot and parts of the paraocular area along the clypeus margin white; flagellum segment 2 longer than half of segment 3; S8 Fig. 1i punctatus (BRULLÉ)

- Clypeus spotted in the middle or black, paraocular area black; flagellum segment 2 clearly less than half as long as segment 3; S8 Fig. $1 \mathrm{~h}$ cypricola (WARNCKE)

5 Mesopleura frontal edge lamellate up to the calli

- Mesopleura frontal edge rounded or edged, not lamellate

6 Scape black; the three apical segments of flagellum also darkened below; mask yellow; mesonotum with moderately dense punctation, silky shiny; S8 Fig. 1a alpinus (MORAWITz)

- Scape usually white spotted; flagellum lightened below to the end; mask white; mesonotum with strong dense punctation, smooth and shiny

7 Scape little expanded, about as wide as the flagellum; malar space much shorter than wide; S8 Fig. 1b

hyalinatus SMITH

- $\quad$ Scape more expanded, wider than the flagellum; malar space longer, about as long as wide; S8 Fig. 1d tauricus (WARNCKE)

8 Malar space long, slightly longer than the diameter of the flagellum; supraclypeal area in profile hardly raised above the level of the frons; face generally flattened; scapes black; S8 Fig. 1k planulus (WARNCKE) Fig. 15

- Malar space shorter than the diameter of the flagellum; supraclypeal area normal, elevated in profile above the level of the frons; face convex; scapes often in large part white spotted

9 Scape long and narrow, about 2.5 times as long as wide; terminal area of the propodeum not or only weakly bordered

- Scape expanded, at most twice as long as broad; terminal area of the propodeum sharply edged around the rim 
10 Scape with white dorsal spot; terminal area of the propodeum surrounded by a ledge; S8 Fig. If

armeniacus (WARNCKE)

- Scape with white longitudinal stripe on the outer margin; terminal area of the propodeum rounded, without ledges to the lateral areas; S8 Fig. 1c adspersus (ALFKEN)

11 Head longer than broad, trapezoidal; lateral spots long, extending beyond the antennal sockets; scape white-spotted; S8 Fig. 11 iranicus DATHE

- Head clearly broader than long, transversely elliptic; lateral spots truncated at top, do not extend beyond antennal sockets; scape bulbous, black; S8 Fig. 1e irritans DATHE

\section{Females}

1 T1 red; tibiae completely yellow, also clypeus richly yellow-spotted sulphuripes (GRIBODO)

- Terga, tibiae (partially) and clypeus black; the latter sometimes with small yellow spot

2

2 Mesopleura with very coarse subcontiguous punctation, the punctures pitlike, their bottom visible; terminal area surrounded by sharp edge

- Mesopleura with strong subcontiguous or dense punctation, the punctures not pitlike; terminal area various ...... 5

3 Malar space longer, slightly longer than the largest diameter of the flagellum

- Malar space shorter than the largest diameter of the flagellum cypricola (WARNCKE)

4 Lateral spots usually reduced, point-like; punctation of the mesosoma coarser, matt punctatus (BRULLÉ)

- Lateral spots filling the paraocular area, reaching above the antennal sockets; clypeus often with spot; punctation of the mesosoma fine, silky shiny longimacula (ALFKEN)

5 Supraclypeal area rhombic, elevated above the frons and converging up into the frons

- Supraclypeal area with parallel sides, clearly flattened and merging fluently into the frons planulus (WARNCKE) Fig. 15

6 Mesopleura anterior edge in upper part sharp-edged; malar space long, nearly as long as wide, clearly longer than the largest diameter of the flagellum

- Mesopleura anterior edge rounded in upper part; malar space shorter, at most half as long as wide, no longer than the largest diameter of the flagellum

7 Undersides of terminal segments of the flagellum black; mesonotum with moderate dense punctation, matt ...... alpinus (MoraWITz)

- Undersides of terminal segments of the flagellum yellow; mesonotum with strong dense punctation 8

8 Mesopleura shagreen, matt; malar space shorter, about three quarters as long as wide hyalinatus SMiтH

- Mesopleura polished, glossy; malar space longer, as long as wide tauricus (WARNCKE)

9 Supraclypeal area widened, upper part nearly circular; head transverse oval; malar space narrow, as long as the flagellum diameter; mesopleura anterior edge ventrally rounded irritans DATHE

- Supraclypeal area upper part narrow; head trapezoidal; malar space longer; mesopleura anterior edge ventrally edged

10 Clypeus shagreen, punctation barely visible amongst sculpture, dull; T1 with distinct fine punctation; lateral spots small, placed low in paraocular area, or absent adspersus (ALFKEN)

- Clypeus glossy, punctation clearly visible; T1 impunctate or with minute punctation; lateral spots filling the paraocular areas, or slightly reduced

11 Lateral spots filling the paraocular area; T2 with very fine dense punctation iranicus DATHE

- Lateral spots reduced below, adjacent to orbits; T2 only with obsolete minute punctation 


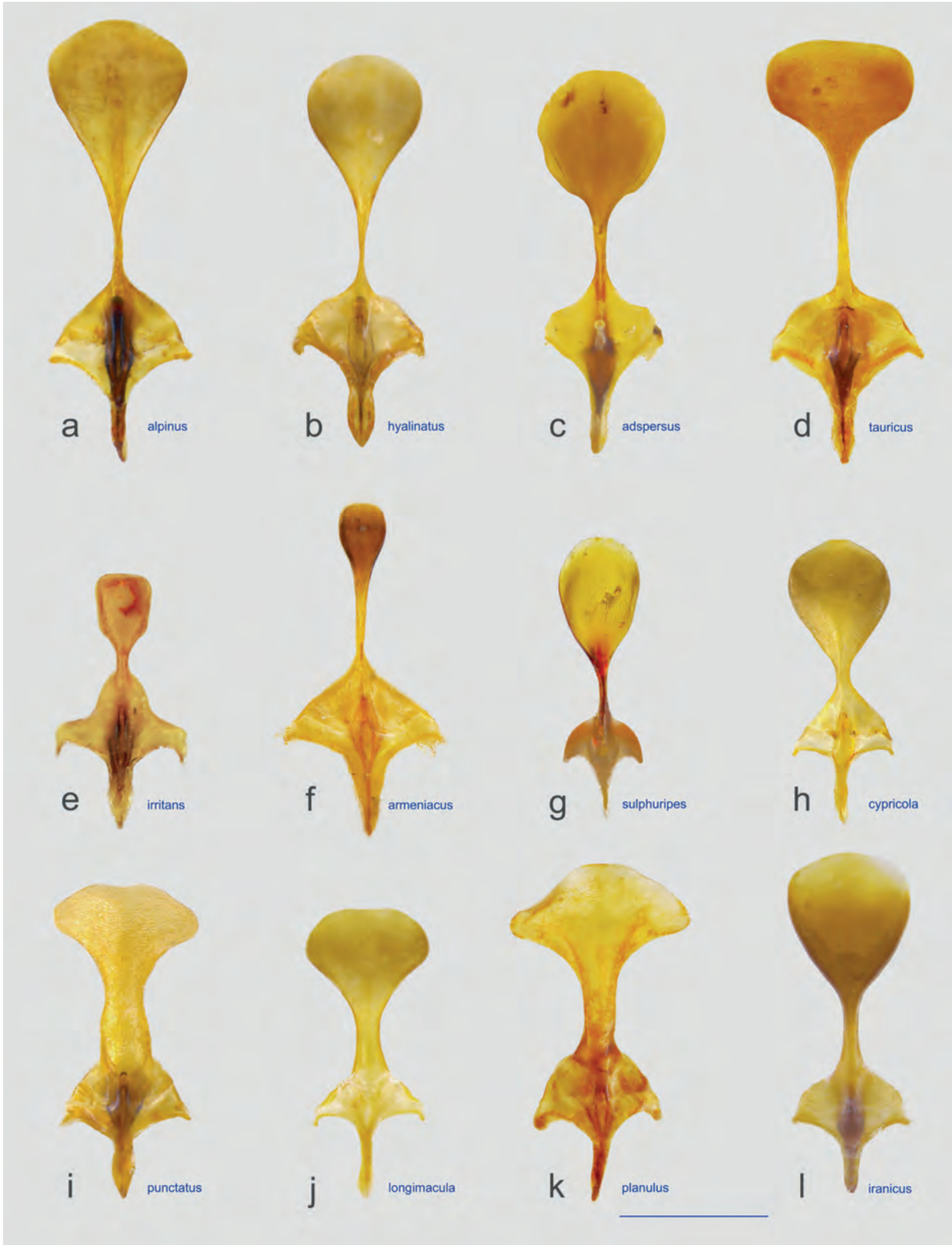

Fig. 1: Male Sternum 8 of the species of subgenus Spatulariella: a-H. alpinus (MorawITz, 1867) (GR Florina); b-H. hyalinatus Smith, 1842 (GR Ioannina); c-H. adspersus (Alfken, 1935); d-H. tauricus (WARncke, 1981), e-H. irritans Dathe, 1980; f-H. armeniacus (WARNCKE, 1981); g-H. sulphuripes (GRIBodo, 1894); h-H. cypricola (WARNCKe, 1972) (CY Girne); i-H. punctatus (Brullé, 1832) (TR Konya); j-H. longimacula (Alfken, 1936) (TR Yüksekova); k-H. planulus (WARnCKe, 1981); 1-H. iranicus Dathe, 1980. - Scale bar $0.5 \mathrm{~mm}$. 


\section{Records}

\section{Subgenus Abrupta MÉHEL̈̈, 1935}

\section{Hylaeus (Abrupta) cornutus CuRTIS, 1831}

Hylaeus cornutus CurTis, 1831: pl. 373, ơ. England: Suffolk. Prosopis (Abrupta) cornuta (SMith, 1842) - MÉHely 1935: 138 WARNCKE 1972: 751.

Hylaeus (Abrupta) cornutus CurTis, 1831 - Aliev 1986: 269.

Material examined: Adana: Aladă̆, 780 m, 02.07.1995, 2 우; leg. Y. Barbier (on Hippomarathrum microcarpum); Hacıhasanlı, NW Sevinçli, 120 m, 07.07.1995, $2 \sigma^{\star} o^{\star}, 3$ ㅇ 우, leg. Y. Barbier (on Ammi visna). Adıyaman: Nemrut dağı, National park, Karadut, 02.07.1993, †, leg. M. Halada (coll. Schwarz/Ansfelden). Ağrı: 3944N $43^{\circ} 03,27.06 .1993,80^{\top} o^{\star}, 2$ 우 우, leg. Jirousek/Halada (coll. Schwarz/Ansfelden). Aksaray: $25 \mathrm{~km}$ SE Ihlara, $38^{\circ} 14 \mathrm{~N}$ $34^{\circ} 18 \mathrm{E}, 18.07 .1998,3$ 우 우, Esmakaya, $38^{\circ} 13 \mathrm{~N} 33^{\circ} 22 \mathrm{E}$, 16.07.1998, ơ, leg. C. Schmid-Egger. Antalya: Alanya, $50 \mathrm{~m}, 28.07 .1985, o^{\star}$, + , R. Hensen; $10 \mathrm{~km} \mathrm{~W}$ Alanya, $36^{\circ} 58 \mathrm{~N} 31^{\circ} 89 \mathrm{E}, 01.08 .2009,10$ 우 우, leg. C. SchmidEgger (shrubland); Arapsuyu, Azmak, 10 m, 24.09.2004, $\sigma^{*}$, + , leg. H. Özbek (on Mentha longifolia); Gazipaşa, $30 \mathrm{~m}, 03.07 .1986,2 \sigma^{\star} o^{\star}$, leg. P. van Ooijen. Balıkesir: Ören, $39^{\circ} 33 \mathrm{~N} 27^{\circ} 02 \mathrm{E}, 04.08 .1987,4$ 우 우, H. Dollfuss. Bitlis: Ahlat, 1750 m, 15.08.1985, 2 ㅇ 우 ; Tatvan, $1750 \mathrm{~m}$, 16.08.1985, 우, leg. R. Hensen. Bolu: lake env, $40^{\circ} 44 \mathrm{~N}$, 3137E, 21.06.1993, ơ , leg. M. Halada. Denizli: Pamukkale, $37^{\circ} 56 \mathrm{~N} 29^{\circ} 08 \mathrm{E}, 14.07 .1998$, o leg. C. Schmid-Egger. Diyarbakır: Dicle University Campus, 700 m, 01.06.2002, 2 ㅇ ㅇ, leg. H. Özbek. Edirne: Keşan, Mecidiye, 50 m, 22.07.2017, $2 \sigma^{\star} o^{\star}$, leg. Ö. Çalmaşur. Erzurum: Atatürk University Campus, $1900 \mathrm{~m}, 09.06 .2005,2$ ㅇ ㅇ, leg. H. Özbek (on Melilotus officinalis; Palandöken, 22002400 m, 28.07.1986, 우 leg. A.W. Ebmer; İspir yolu, 2 km S of Dallıkavak Geçidi, 2300 m, 30.07.2010, 2 우 우, leg. J.S. Ascher, H. Özbek (coll. AMNH); Horasan 18 km E Delibaba, $40^{\circ} 02 \mathrm{~N} 42^{\circ} 09 \mathrm{E}, 25.06 .1993$, 우 , leg. Halada; Narman, Tahtakale, $1350 \mathrm{~m}, 17.08 .2004,2$ ㅇ 우, leg. S. Çoruh; Oltu, Çamlıbel, $1600 \mathrm{~m}, 26.07 .2000,2$ 우 우 leg. H. Özbek (on Melilotus alba); Uzundere, Gölbaşı,

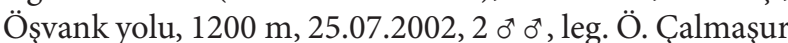
(on Daucus carota). Hakkâri: 1750 m, 09.07.1987, o`, leg. R. Hensen; Varegöz, $37^{\circ} 25 \mathrm{~N} 44^{\circ} 13 \mathrm{E}, 1650.02 .08 .1986$, ơ leg. S.M. Blank. Iğdır: Bayraktutan, 900 m, 17.08.2005, 8 ㅜ, leg. H. Özbek (on Centaurea solstitialis); Köy Hizmetleri Araştırma Enstitüsü, 900, 31.07.2002, 6 우 우, leg. M. Kesdek (on M. alba); Melekli, 925 m, 16.08.2005, 2 우 이 leg. H. Özbek. İzmir: Selçuk, Efes, 18.05.1992, 우, leg. W.H. Liebig. Kahramanmaraş: Göksün, 1400 m, 26.06.1988, 3 ㅇ ㅇ , leg. R. Hensen. Kars: 25 km ESarıkamış, $2100 \mathrm{~m}, 06.07 .1985$, o ${ }^{\star}$, leg. C.J. Zwakhals; Karakurt, $20 \mathrm{~km} \mathrm{~W}, 40^{\circ} 10 \mathrm{~N} 42^{\circ} 22 \mathrm{E}, 1600 \mathrm{~m}, 04.07 .1985,2 o^{\star} o^{\star}$, ㅇ, leg. M. Schwarz (coll. Schwarz/Ansfelden); Kağızman, Değirmendere, 1350 m, 04.06.2004, ơ, M. Kesdek (on Eryngium campestre). Konya: $60 \mathrm{~km} \mathrm{W,} \mathrm{Eflatun} \mathrm{Pınarı,}$

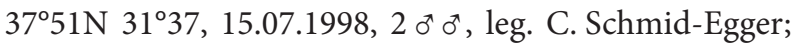

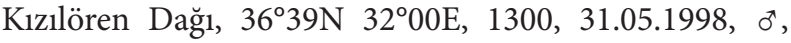
leg. S.M. Blank. Kütahya: Sobran, Porsuk Baraj1, 39 $40 \mathrm{~N}$ $30^{\circ} 10 \mathrm{E}, 08.07 .1993,2$ 우 잉 leg. Jirousek (coll. Schwarz/ Ansfelden). Mersin: Silifke, Kargıcan, 200 m, 04.08.1985, + , leg. R. Hensen. Muğla: Bodrum, Salmakis, $37^{\circ} 02 \mathrm{~N}$ $27^{\circ} 25 \mathrm{E}, 14.07 .2001$, 오 F. Burger (Polygonum sp.); Milas

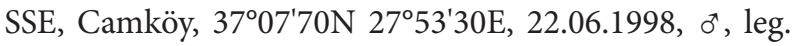
C. Niehuis. Nevşehir: 2 km S, 1250 m, 04.07.1984, ㅇ, leg. A.W. Ebmer; Acıgöl, Cardak, 38³3N 34²47E, 07.07.1993,

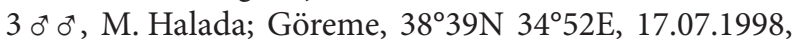
ơ, leg. Jirousek; Topuzzdağ 1 Pass W, 1300 m, 17.07.1984, ㅇ, leg. A.W. Ebmer; Avanos, Zelve, 1050 m, 18.07.1984, $\sigma^{*}$, 04.07.1984, ㅇ, leg. A.W. Ebmer; Ürgüp, 1100 m, 11.08.1985, $3 \sigma^{\top} \sigma^{\star}$, leg. P. van Ooijen. Niğde: Karakışlakçı, 1240, 07.07.1995, ơ, ๆ, Y. Barbier (on Euphorbia altissima). Şanlıurfa: Halfeti, $400 \mathrm{~m}, 28.06 .1987,2 \sigma^{\top} o^{\star}$, 2 우 오, leg. R. Hansen. Van: $38^{\circ} 30 \mathrm{~N} 43^{\circ} 24 \mathrm{E}, 28.06 .1993$, $\sigma^{\star}$, leg. Jirousek (coll. Schwarz/Ansfelden); $1800 \mathrm{~m}$, 13.07.1987, ơ, leg. R. Hansen. Yalova: 4 km E Çiftlik köyü, 50 m, 31.07.1986, ㅇ, leg. P. van Ooijen.

Remarks: H. cornutus is widely distributed in Turkey and not rare. Data from the following provinces had hitherto been registered: Amasya, Ankara, Bursa, Çanakkale, Denizli, Hatay, İstanbul, İzmir, Kırıkkale, Kütahya, Mersin, Osmaniye, Tekirdağ, Yalova (WARNCKe 1972), Erzurum and Kars (ÖzBeK 1977). With our new data from 20 provinces, the species has so far been confirmed from 36 provinces of Turkey. $H$. cornutus lives from the lowlands in dry-warm habitats up to cool environments at $2400 \mathrm{~m}$ altitude. The flight period is quite long, it extends from May to September with a peak in July, so that more than one generation is conceivable. Flower visits to the following plants have been registered: Hippomarathrum microcarpum, Ammi visnaga, Centaurea solstitialis, Daucus carota, Euphorbia altissima, Melilotus alba, Melilotus officinalis, Mentha longifolia, Polygonum sp.

Distribution: Nearly circum-mediterranean, from Spain in the west to Turkmenistan in the east; north to Denmark, south to Israel (DATHE et al. 2016; DATHE \& ProshChalykin 2018).

\section{Subgenus Dentigera Popov, 1939}

\section{Hylaeus (Dentigera) acer DATHE, 1980}

Hylaeus (Dentigera) acer DATHE, 1980: 80-81, ơ ㅇ. Iran: Elburs, Damawand area, $2200 \mathrm{~m}$.

Prosopis (Nesoprosopis) acra (DATHE, 1980) - WARNCKE 1992: 771.

Material examined: Hakkâri: Beytüşşebap, Habur Deresi, $37^{\circ} 32 \mathrm{~N} 43^{\circ} 12 \mathrm{E}, 1100 \mathrm{~m}, 10.08 .1983$, ơ , ㅇ, leg. W. Schacht (coll. Schwarz/Ansfelden); Varagöz, 37²5E $44^{\circ} 13 \mathrm{~N}, 1650$ m, 02.08.1986, ơ , leg. S.M. Blank; Varagöz, 
Sat Dağ $1,37^{\circ} 25 \mathrm{E} 44^{\circ} 13 \mathrm{~N}, 1700 \mathrm{~m}, 04.08 .1983,3$ ㅇ 운, leg. W. Schacht (coll. Schwarz/Ansfelden); Yüksekova, $30 \mathrm{~km}$ W, $1850 \mathrm{~m}, 19.07 .1986,2$ 우, ㅇg. A.W. Ebmer. Tunceli: Ovacık $30 \mathrm{~km} \mathrm{W,} 1250$ m, 19.08.1985, 2 우 우, leg. R. Hensen.

Remarks: The male is richly marked with pale, the scape strongly thickened, sternum 3 with narrow, pointed spine. The propodeum medial area is longitudinally striped in females and males. The species is only known from the mountains of Eastern Anatolia and Northern Iran, where it was found at altitudes between 1100 and $3000 \mathrm{~m}$. It has been collected in Turkey only sparsely (3 provinces); the record in Tunceli is new.

Distribution: Turkey: Hakkâri, Siirt (WARNCKE 1992), Tunceli; Iran: Elburs (DATHE 1980).

\section{Hylaeus (Dentigera) brevicornis NyLANDER, 1852}

Hylaeus brevicornis NylANDER, 1852: 95, 우 ơ. Sweden. Prosopis (Nesoprosopis) brevicornis seducta (Förster, 1871) WARNCKE 1972: 765.

Hylaeus (Dentigera) brevicornis Nylander, 1852 - Aliev 1986: 264.

Material examined: Ardahan: Gölebert, $2000 \mathrm{~m}$, 10.08.1976, ㅇ, ơ, leg. H. Özbek (Carduus nutans). Artvin: Genya Mt, 1860, 28.07.2004, ơ, leg. C. Güçlü; Murgul, 01.07.1997, 2 o $^{\star}$, leg. Prudek, Riha. Balıkesir: Erdek, Halbinsel, 40²4N 2747E, 03.08.1986, ơ, leg. Madl (coll. Schwarz/Ansfelden). Bingöl: Genç, 1000 m, 12.08.1985, o $0^{\star}$, leg. R. Hensen. Bolu: $40^{\circ} 44 \mathrm{~N}, 31^{\circ} 37 \mathrm{E}$, 21.06.1993, ㅇ, leg. M. Halada (coll. Schwarz/Ansfelden). Denizli: Pamukkale, 07.08.1986, ㅇ, leg. Kadlec/Vorisek. Erzincan: Rafahiye, $8 \mathrm{~km} \mathrm{~N}, 1700 \mathrm{~m}, 15.07 .1984$, ơ, leg. A.W. Ebmer. Erzurum: Oltu, Başaklı, 1700 m, 13.08.1976, ?, leg. H. Özbek. Kayseri: Develi, Bakırdağ 1,1700 m, 06.07.1984, ơ , leg. A.W. Ebmer. Muș: Buğlan Pass, 40 km W Muş, 1640 m, 08.07.1985, ơ, leg. A.W. Ebmer. Nevşehir: Ürgüp, Topuz Dağı Geçidi W, 1300 m, 17.07.1984, $20^{\pi} 0^{*}, 4$ 우 우, leg. A.W. Ebmer; Avanos, Zelve, 1050 m, 04.07.1984, +, leg. A.W. Ebmer. Sivas: Gürün, Suğul, 1700 m, 31.07.1986, ơ, leg. A.W. Ebmer; Yıldızeli, Çamlıbel Pass, 1600-1700 m, 16.07.1984, ơ , leg. A.W. Ebmer. Van: 1800 m, 13.07.1987, o, leg. R. Hensen; Başkale, Güzeldere Pass, 2600-2800 m, 19.07.1986, 우, leg. A.W. Ebmer.

Remarks: The taxonomy of the Hylaeus brevicornis group is still difficult and not finally clarified. However, Warncke's concept of a single polymorphic species has been refuted by recent molecular genetic findings. We treat here the taxa $H$. acer, H. brevicornis, H. gredleri, $H$. imparilis, $H$. intermedius, $H$. kahri and $H$. punctus on the basis of morphological characters as independent species.
The species is widespread throughout the country (31 provinces) and very common. The flight season runs from the end of June to almost the end of August.

Distribution: Palaearctic: North Africa (Morocco), Europe, South Siberia, Kazakhstan. In Turkey: Adana, Amasya, Ankara, Antalya, Bursa, Denizli, Hatay, İstanbul, İzmir, Kastamonu, Kayseri, Konya, Kütahya, Mersin, Muğla, Nevşehir, Şanlıurfa, Tekirdağ, Trabzon (WARNCKe 1972); Erzurum (ÖzBEK 1977). The records from the following 12 provinces are new: Ardahan, Artvin, Balıkesir, Bingöl, Bolu, Denizli, Erzincan, Kayseri, Muş, Nevşehir, Sivas, Van.

\section{Hylaeus (Dentigera) chukar (WARNCKE, 1992)}

Fig. 2

Prosopis (Nesoprosopis) chukar WARNCKE, 1992: 771-772, 799, i $0^{\top}$. Turkey: Hakkâri.

Hylaeus (Dentigera) chukar (WARNCKE, 1992) - ITIS (2020).

Remarks: The assignment of the species to the subgenus Dentigera is problematic. Despite some similarity, the species does not belong to the brevicornis-group at all; the terminalia of the male also resemble some species of the subgenus Prosopis; it remains enigmatic in this respect. WARNCKE (1992: 767) placed it between H. acer and $H$.duckei, representatives of subgenera Dentigera and Prosopis in our sense.

A special character of both sexes is the widened supraclypeal area; its apical part is as broad as long, in the male even broader; it merges evenly and broadly into the frons. : : scape black, flagellum short; facial side spots small; tergum 1 smooth and glossy, without cross-ripple. $\sigma^{*}$ scape slender with bright apical spot; the mask white; apical lobes of sternum 7 at the outer edge with long fine bristles (no teeth); sternum 8 short, in front with $\mathrm{V}$-incision (artefact?); the penis valves are only slightly curved, the space in between narrow as in H. acer.

The species name "chukar" is arbitrarily and unrelatedly conveyed from the name of the chukar partridge (Alectoris chukar), a Eurasian upland gamebird in the family Phasianidae, which got its name after its sound. According to the application of Warncke we understand the epithet as an apposition of the noun in the nominative.

The species is rarely recorded from Turkey ( 1 province). Despite intensive collections throughout the country, no additional samples have been taken. Since H. chukar is a high mountain species (1800-3000 m), further collections at higher altitudes (over $2000 \mathrm{~m}$ ), especially in Eastern Anatolia, should be carried out throughout the season.

Distribution: Turkey: Hakkâri (WARNCKE 1992: 772). 


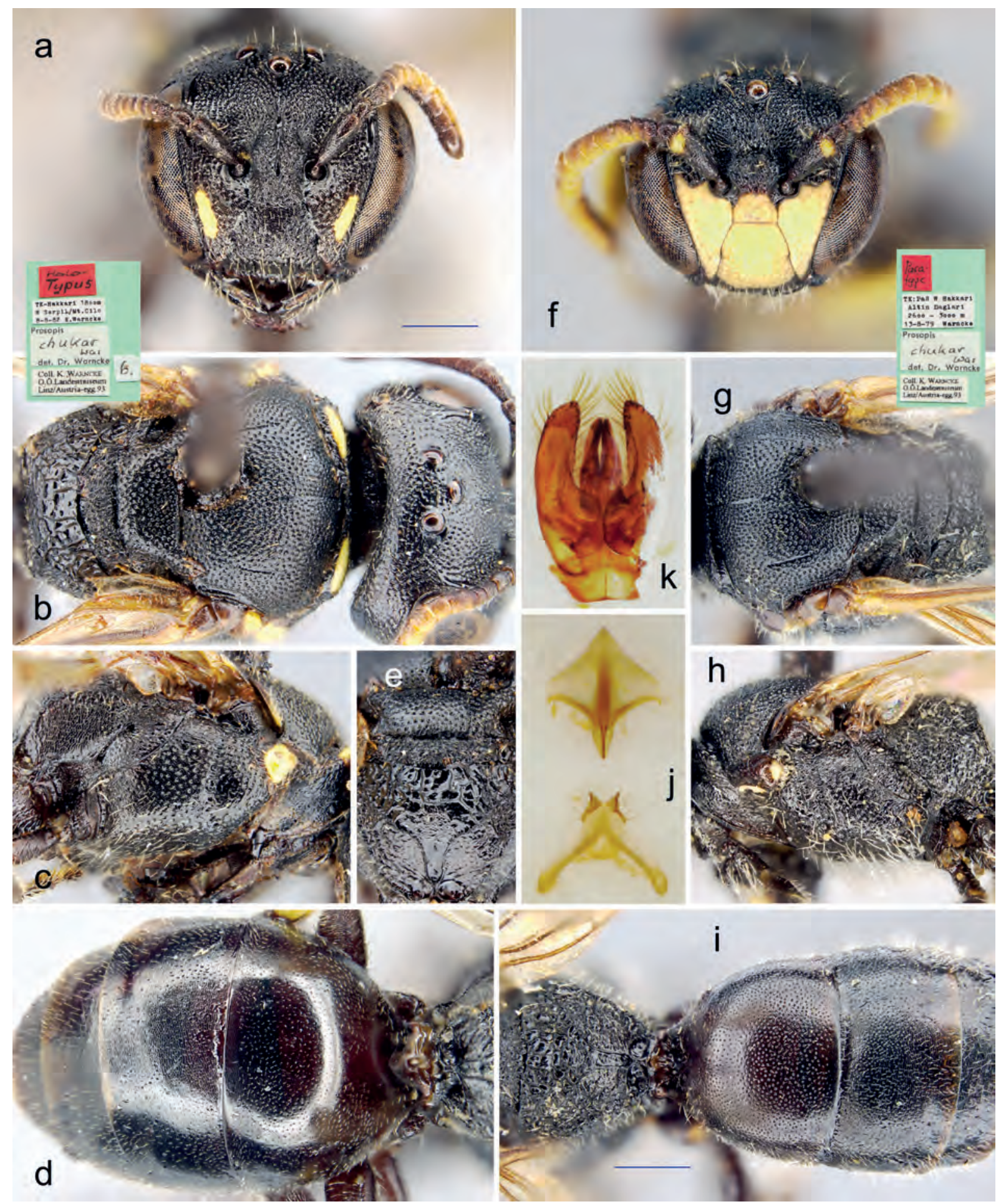

Fig. 2: H. (Dentigera) chukar (WARncKe, 1992). Holotype female: a-face, b-mesonotum, c-mesopleuron, d-propodeum, e-metasoma. Paratype male: f-face, $\mathbf{g}$-mesonotum, h-mesopleuron, i-propodeum and metasoma, j-S7 and S8, k-genital capsule. - Scale bar $0.5 \mathrm{~mm}$.

Hylaeus (Dentigera) giresunus (WARNCKE, 1992) Fig. 3

Prosopis (Nesoprosopis) glacialis giresuna WARNCKE, 1992: 770-771, ㅇ o $o^{7}$. Turkey: Hakkâri.

Hylaeus (Dentigera) giresunus (WARNCKE, 1992) - comb. et stat. nov.
Remarks: The female of $H$. giresunus (holotype) differs from $H$. glacialis by the clypeus almost completely concave throughout its length, while in the latter only the clypeus front edge is transversely impressed. Additionally, in the female the front margin of the clypeus has an arcuate tip. The outline of the head is sub-quadratic (H. glacialis: trapezoidal) and the genae are thickened 
(normal in H. glacialis). The habitus of $H$. giresunus is much more slender, especially the tergum 1, which is strongly shiny; the sculpture of the propodeum is less pronounced. The males are very similar, also in the terminalia, but the proximal extended apical lobes of sternum 7 may be important. H. giresunus is certainly a geographical parallel to the European $H$. glacialis, but the female appears to be discrete from it.

H. giresunus is a montane (at $1700-2400 \mathrm{~m}$ ), sparsely recorded species ( 3 provinces).

Distribution: Turkey: Hakkâri, Giresun, Erzurum (WARNCKE 1992: 771).

\section{Hylaeus (Dentigera) glacialis MoraWITz, 1872}

Hylaeus glacialis Morawitz, 1872: 379, ơ . South Tyrol: Trafoi. Hylaeus (Dentigera) glacialis Morawitz, 1872 - Dathe et al. 2016: 15,45 .

Material examined: Erzurum: Oltu, Başaklı, Karadağ Mt, 2100 m, 24.07.2005, ơ, leg. H. Özbek (on Eryngium billardieri); Çamlıbel Karakolu, 1900 m, 01.07.2000, o, leg. H. Özbek; Tortum/Narman Kireçli Dağı Geçidi W, 2100 m, 14.07.1984, ơ, A.W. Ebmer. Hakkâri: Varagöz, $37^{\circ} 25 \mathrm{~N} 44^{\circ} 13 \mathrm{E}, 1650 \mathrm{~m}, 02.08 .1986$, 우, leg. S. M. Blank; Oramar, $10 \mathrm{~km} \mathrm{NE}, 1700 \mathrm{~m}, 29.06 .1985$, ㅇ, leg. M. Schwarz.

Remarks: It is remarkable that both taxa $H$. giresunus and $H$. glacialis are said to occur side by side in two provinces (Erzurum, Hakkâri). Errors in older determinations cannot be completely ruled out here. To answer this question, females will have to be compared.

Distribution: Has a disjunctive range in high mountain locations (1500-2100 m) of the Pyrenees, Alps, the Balkans and Asia Minor. Turkey: Erzurum, Hakkâri (Fig. 17.II).

\section{Hylaeus (Dentigera) gredleri FöRSTER, 1871}

Hylaeus gredleri Förster, 1871: 944-946, ㅇ o ${ }^{\star}$. Austria: Telfs/ Tirol.

Hylaeus (Dentigera) gredleri Förster, 1871. - Aliev 1986: 264. DATHE et al. 2016: 17, 45.

Material examined: Ağrı: 10 km S, 1650 m, 26.07.2003, 6 우 우, leg. H. Özbek; Cumaçay 1930 m, 23.07.1996, + , leg. P. Rasmont (on Cirsium arvense vestitum) ; Hamur, 26.07.2003, 2 ㅇ ㅇ, leg. H. Özbek. Erzurum: 5 km SW, 27.07.2010, o, leg. J. Ascher, H. Özbek; Atatürk University Campus, 2000 m, 11,07.2007, ơ, leg. leg. Ros. Asch Özb, 18.08.1970, ơ, leg. H. Özbek; Oltu, Başaklı, $1500 \mathrm{~m}, 20 \mathrm{~km}$ WSW of Oltu, $29^{\circ} 14 \mathrm{~N}$ $41^{\circ} 48 \mathrm{E}, 03.07 .2001, o^{\star}, 4$ 우, leg. J.G. Rozen \&
H. Özbek (coll. AMNH); Başaklı, Karadağ, 2100 m, 24.07.2005, 2 ㅇ ㅇ, H. Özbek (on Eryngium creticum); Çamlıbel, $1750 \mathrm{~m}, 40.28 .22 \mathrm{~N}$ 41.46.35E, 11.07.2004, $30^{\top} o^{\star}$, leg. H. Özbek; Pasinler, $5 \mathrm{~km} \mathrm{~W}$ of Pasinler, 10.07.2007, ơ , leg. Ros. Asch Özbek (coll. AMNH). Hakkâri: Yüksekova, Esendere, 21.07.1998, †, leg. C. Schmid-Egger. İzmir: Efes, 18.05.1992, $3 \sigma^{\star} \sigma^{\star}$, 3 우 우, leg. W.H. Liebig. Kars: Sarıkamış, Akkurt çeșmesi, 1550 m, 19.06.2004, ơ , ㅇ, leg. H. Özbek; Karakurt, $1501 \mathrm{~m}, 40^{\circ} 08 \mathrm{~N} 42^{\circ} 21 \mathrm{E}, 05.08 .2002,3$ 우 우, leg. H. Özbek (on Achillea millefolium L.). Kırşehir: Kulpak, $38^{\circ} 27 \mathrm{~N} 34^{\circ} 14 \mathrm{E}$, 우, 28.05.2001, 우, 02.06.2002, leg. W.H. Liebig. Konya: $25 \mathrm{~km} \mathrm{N,} \mathrm{11.06.1966,} 2$ 우 우, leg. HHF Hamann; Beyşehir, 13.06.1966, ơ; Dedegöl Dağı, 1400-1700 m, 15.06.1966, ơ, leg. HHF Hamann. Kahramanmaraș: Göksun, 1400 m, 26.06.1987, $4 o^{\star} \sigma^{\top}$, leg. R. Hensen. Mersin: Mut $10 \mathrm{~km} \mathrm{S,} \mathrm{08.06.1966,} \mathrm{우,}$ leg. HHF Hamann. Nevşehir: Avanos, $38^{\circ} 46 \mathrm{~N} 34^{\circ} 54 \mathrm{E}$, 25.05.2001, †, leg. W.H. Liebig; Göreme, 1000 m, 09.07.1988, 3 우 우, 우, leg. C. Schmid-Egger. Niğde: Yeniköy, 1370 m, 07.07.1995, đ, leg. Y. Barbier (on Euphorbia altissima); Karakışlakcı, 1240 m, 07.07.1995, $5 \sigma^{*} \sigma^{*}, 10$ 우 ㅇ, leg. D. Flagothier, Y. Barbier (on Astrodaucus orientalis, Tortilis uranica).

Remarks: The available records suggest that $H$. gredleri occurs mainly in Eastern and Central Anatolia. It preferentially inhabits steppes, open shrubby habitats and forest margins from sea level to altitudes of $2000 \mathrm{~m}$. The flight season extends from the end of May to the end of August, with a peak in June. Flowers visited: Achillea millefolium, Astrodaucus orientalis, Euphorbia altissima, Eryngium creticum, Tortilis uranica. The species is moderately recorded in Turkey (11 provinces).

Distribution: All Europe south to Sicily, up to $1500 \mathrm{~m}$, Russia, and from western Morocco, Lebanon and Azerbaijan to Kazakhstan. Turkey: 11 provinces; older data are not usable as the species was previously mixed with H. brevicornis.

\section{Hylaeus (Dentigera) imparilis FöRSTER, 1871}

Hylaeus imparilis FöRSTER, 1871: 1033-1035, ơ. Southern France.

Hylaeus (Dentigera) imparilis Förster, 1871 - Aliev 1986: 264. DATHE et al. 2016: 17, 44

Material examined: Ağri: $39^{\circ} 44 \mathrm{~N} 43^{\circ} 03 \mathrm{E}, 27.06 .1993$, $0^{\star}, 2$ 우 우, leg. Jirousek/Halada (coll. Schwarz/Ansfelden). Aksaray: Ihlara, $38^{\circ} 14 \mathrm{~N} 34^{\circ} 18 \mathrm{E}, 18.07 .1998$, ơ, leg. C. Schmid-Egger. Ankara: 09-16.06.1934, ơ, H. Noack (Museum Senckenberg). Antalya: $33 \mathrm{~km}$ NW, $37.02 \mathrm{~N}$ 30.23E, 23.07.1998, $2 \sigma^{\star} \sigma^{\star}$, leg. C. Schmid-Egger; Alanya, 50 m, 28.07.1985, $4 \sigma^{\star} o^{\star}, 6$ 우 ㅇ, leg. R. Hensen; Demirtaş, 100 m, 29.07.1985, ơ , 4 우 ㅇ, leg. R. Hensen; Seki, 10.09.1951, ㅇ, leg. H.A. Guenin; İncekum, 20 m, 


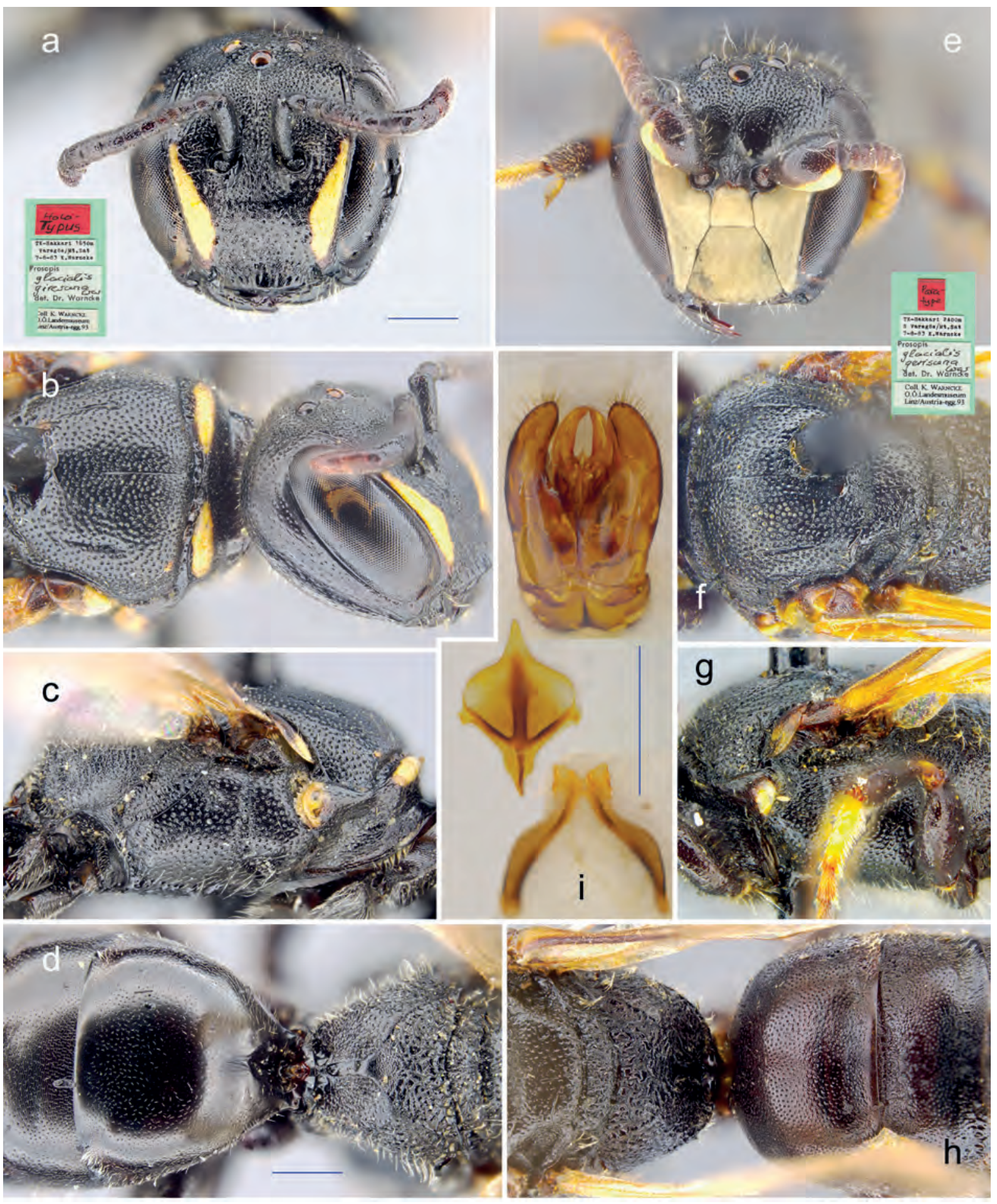

Fig. 3: H. (Dentigera) giresunus (WARNCKe, 1992). Holotype female: a-face, b-mesonotum, c-mesopleuron, d-propodeum and metasoma. Paratype male: e-face, $\mathbf{f}$-mesonotum, $\mathbf{g}$-mesopleuron, $\mathbf{h}$-propodeum and metasoma, $\mathbf{i}$-terminalia. - Scale bar $0.5 \mathrm{~mm}$.

07.08.1985, $4 \sigma^{\star} \sigma^{\star}$, leg. P. van Ooijen; Konaklı, 3658 31 ${ }^{\circ} 89 \mathrm{E}, 01.08 .2009,4$ o $^{\star}, 4$ 우 우 , leg. C. Schmid-Egger (woodland, coll. Schmid-Egger); Akseki, $1200 \mathrm{~m}$, 09.08.1985, 3 우 우, leg. P. van Ooijen; Anamur, $36^{\circ} 02 \mathrm{~N}$ 32 79E, 21.07.1998, ㅇ, leg. C. Schmid-Egger; Elmalı, $24 \mathrm{~km} \mathrm{~S}, \quad 1500-1600 \mathrm{~m}, 04.07 .1990$, †, A.W. Ebmer;

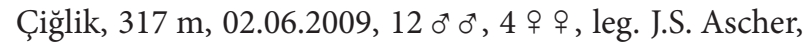
H. Özbek; Kargın, 02.06.2009, 2 o $^{\star}$, leg. J.S. Ascher,
H. Özbek ((coll. AMNH); İbradi, 6 km SE, 450 m, 3707N $31^{\circ} 65 \mathrm{E}, 31.07 .2009,30^{\top} o^{\star}$, 우 , leg. C. Schmid-Egger; Koyaaltı Beach, 2 m, 29.05.2009, $70^{\star} o^{\star}$, leg. J.S. Ascher, H. Özbek, J.G. Rozen (coll. AMNH); Kumluca, Arıkanda, $36^{\circ} .28 \mathrm{~N} 30^{\circ} 07 \mathrm{E}, 11.07 .1998,0^{\top}$, ㅇ, leg. C. Schmid-Egger; Side, 06-20.06.1985, 2 우 우, leg. N. Mohr; Manavgat, $50 \mathrm{~m}, 31.07 .1985$, ơ, leg. R. Hensen; Termessos, 2 우 ㅇ, 07.07.1998, leg. C. Schmid-Egger; Güllük Dağ 1 


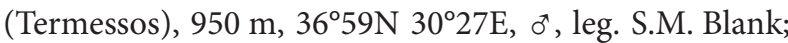
Kaş, Kalkan, 13.09.1951, 2 우, leg. H.A. Guenin; Kemer, 08.09.1951, ㅇ, leg. H.A. Guenin. Artvin: Borçka, Ferhatl, 21.05.2002, $70^{\star} 0^{\star}, 3$ 우 ㅇ, leg. J.G. Rozen and H. Özbek (coll. AMNH); Yusufeli, Demirkent, Salekör, 1600 m, 01.09.1995, o leg. M. Kraus. Aydın: Çamlık, 3753N 2726E, 17.05.1981, o`, leg. Rausch/Ressl, (coll. Mus. Linz) Çine, 20-23.09.1951, + , leg. H.A. Guenin. Balıkesir: Ayvalık, Arkent, 28.06.1993, ㅇ, leg. P. Tyrner. Bingöl: Genç, 1000 m, 12.08.1985, ơ, leg. R. Hensen; Genç $15 \mathrm{~km} \mathrm{S,} 1400 \mathrm{~m}, 13.08 .1985,3 \sigma^{*} \sigma^{*}$, leg. R. Hensen. Bitlis: Ahlat, 1750, 18.08.1990, ơ , leg. R. Hensen. Burdur: Çeltikçi, 02.09.1983, 2 o $^{\star} \sigma^{*}, 2$ 우 잉. leg. P v Ooijen, J. Timmer. Bursa: 250 m, 24.08.1985, $8 \sigma^{\star} \sigma^{\star}, 2$ 우 우, leg. R. Hensen; Mudanya: 25 m, 25.08.1985, $2 o^{\star} o^{\star}$, + , leg. R. Hensen. Denizli: Pamukkale 18 km N, 3756N 2908E, 14.07.1998, ㅇ, leg. C. Schmid-Egger. Dıyarbakır: Çınar, 650 m, 09.081.985, $2 \sigma^{\star} \sigma^{\star}$, leg. R. Hensen. Erzincan: Muti köprüsü, 1300 m, 26.09.1979, ㅇ, leg. H. Özbek (on Eryngium campestre); Topdağ1, $1400 \mathrm{~m}, 04.08 .2003$, ơ, leg. S. Çoruh. Erzurum: Atatürk University research field 6 nolu kuyu, 2000 m, 05.09.1966, ơ , leg. H. Özbek (on Melilotus officinalis); Olur, Yeşilbağlar, 1200 m, 25.06.2001, 2 ㅇ ㅇ, leg. H. Özbek, J.G. Rozen; Oltu, Basakli, 18 km WSW of Oltu, $1500 \mathrm{~m}, 29^{\circ} 14 \mathrm{~N} 41^{\circ} 48 \mathrm{E}, 27.06 .2001,2$ 우 우, leg. J.G. Rozen and H. Özbek (coll. AMNH); Subatık, 1300 m, 30.08.2003, $2 \sigma^{\star} o^{\star}$, leg. H. Özbek (on Daucus carota); $20 \mathrm{~km} \mathrm{SE}$ of Tortum, $2131 \mathrm{~m}, 40^{\circ} 21 \mathrm{~N} 41^{\circ} 40 \mathrm{E}$, 21.07.2010, 2 ㅇ 우, leg. J.S. Ascher, H. Özbek, J.G. Rozen (coll. AMNH). Eskişehir: Sakarılıca, 06.07.1997, ơ , + , leg. Prudek/Ríha. Hakkâri: Varagöz, 1750 m, 37²5N 44²13E, 06.08.1986, ơ, S.M. Blank; Şemdinli, 1700, 20.07.1988, ơ, leg. C. Schmid-Egger; Yüksekova, 3725N 4413E, 1700 m, 04-8.08.1983, 13 क o $^{\top} 12$ 우 우, leg. W. Schacht (coll. Schwarz/Ansfelden) Hatay: Belen, 28.08.1983, ㅇ, leg. J. Timmer. Iğdır: $900 \mathrm{~m}, 18.08 .1983,2 \sigma^{\star} \sigma^{\star}$, leg. J. Timmer. Isparta: Eğirdir, 15-25.05.1988, 2 우 우, leg. N. Mohr; $37^{\circ} 53 \mathrm{~N} 30^{\circ} 55 \mathrm{E}, 15.07 .1998$, ㅇ , leg. C. Schmid-Egger (coll. Mus.Linz); $37^{\circ} 48$ N 31 ${ }^{\circ} 07 \mathrm{E}, 13.06 .1985$, o ${ }^{\star}$, leg. H. Rausch; Yalvaç, Sultandağ $1,38^{\circ} 18 \mathrm{~N} 31^{\circ} 09 \mathrm{E}$, $0^{\star}$, leg. Jirousek (coll. Schwarz/Ansfelden). İzmir: Efes, $9 \mathrm{~m}, 18.05 .1992$, ㅇ, W.H. Liebig; Selçuk, Çamlık, 3753N 27²6E, 17.05.1981, ๙ 1700 m, 06.07.1984, ơ, leg. A.W. Ebmer; Pınarbaşı, 1500 m, 25.06.1987, $2 o^{\star} o^{\star}$, + , leg. R. Hensen. Karaman: Sertavul geçidi, 1550 m, 08.07.1990, ơ, leg. A.W. Ebmer. Konya: Güneysınır, Gürağaç, 1020 m, 06.08.2000, 4 우 우, leg. M. Kesdek; Doğanşehir, Reşadiye geçidi, 1500 m, 12.07.1990, $20^{\star} o^{\star}$, leg. A.W. Ebmer; Beyşehir, Efla-

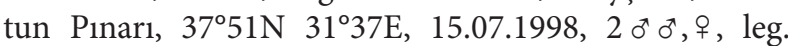
C. Schmid-Egger; Beyşehir, $1150 \mathrm{~m}, 18.06 .1987$, leg. R. Hensen. Kütahya: Porsuk baraj1, Sobran, $39^{\circ} 40 \mathrm{~N}$ $30^{\circ} 10 \mathrm{E}, 08.07 .1993,20^{*} \sigma^{*}, 3$ ㅇ ㅇ, leg. Jirousek/Halada (coll. Schwarz/Ansfelden). Mardin: 1000 m, 02.07.1987, $0^{\star}$, R. Hensen. Mersin: 26.07.1997, ơ , leg. J. Timmer; Gülnar, 1000 m, 31.07.1988, o`, leg. C. Schmid-Egger; Silifke, Uzuncaburç, $400 \mathrm{~m}, 02.08 .1985,2$ 우 우, leg. R. Hensen; Kargicak, 200 m, 14.08.1985, $4 o^{\star} o^{\star}, 5$ ㅇ 우,
R. Hensen; Mut, 11.07.1996, ㅇ, leg. Brechtel/Ehrmann. Muğla: Fethiye, Ölüdeniz, 50 m, 25.07.1986, 4 ㅇ ㅇ, leg. P. van Ooijen; Marmaris, $20 \mathrm{~km} \mathrm{W,} \mathrm{ơ,} 3$ ㅇ ㅇ, leg. A. Link; Marmaris $56 \mathrm{~km}$ NNW, $15 \mathrm{~km}$ E Milas, $37^{\circ} 18 \mathrm{~N} 27^{\circ} 57 \mathrm{E}$, 13.07.1998, ơ, ㅇ , leg. C. Schmid-Egger. Nevșehir: Acıgöl,

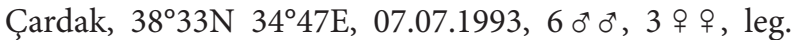
Jirousek (coll. Schwarz/Ansfelden); Avanos, 07.07.1983, $0^{*}$, leg. J. Hladil; Göreme, 38³9N 3452E, 17.07.1998, 3 o $^{\star} \sigma^{\star}$, leg. C. Schmid-Egger; $900 \mathrm{~m}, 09.08 .1988$, ơ , leg.

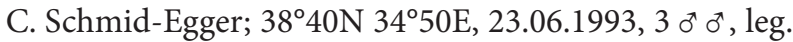
M. Halada (coll. Schwarz/Ansfelden); Ürgüp, Topuzdağ geçidi, 1300 m, 17.07.1984, ơ, leg. A.W. Ebmer; Ürgüp E 10 km, Aksalur W, 1350 m, 38.40N 034.59E, 27.05.1998, ${ }^{\star}$, leg. S.M. Blank. Niğde: Yassıkaya, Elek gölü, 1510 m, 08.07.1995, ơ, leg. Y. Barbier (on Eryngium billardieri).

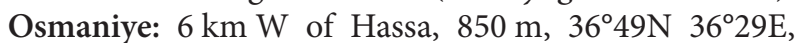
13.05.2002, $5 \sigma^{\star} \sigma^{\star}$, leg. J.G. Rozen and H. Özbek (coll. AMNH); Sivas: Gürün, NW, 1400 m, 13.07.1990, $o^{\star} \sigma^{\star}$, leg. A.W. Ebmer; Koyulhisar, $40^{\circ} 18 \mathrm{~N} 37^{\circ} 50 \mathrm{E}, 19.05 .2007$, $0^{*}$, W.H. Liebig. Şanlıurfa: Suruç, 400 m, 29.06.1987, $4 o^{\top} o^{\star}$, + , leg. R. Hansen; Haffeti, 450 m, 07.08.1985, $160^{\top} o^{\top}, 8$ ㅇ ㅇ , leg. R. Hensen. Van: 1800 m, 13.07.1987, ㅇ, leg. R. Hensen; Gevaş, 3843N 4319E, 29.06.1993, 우 leg. R. Hensen (coll. Schwarz/Ansfelden). Yalova: Çiftlik köyü, 50 m, 31.07.1986, 5 ơ o , 16 ㅇ , leg. P. van Ooijen; Termal, 300 m, 28.08.1985, ơ , leg. R. Hensen.

Remarks: According to our compilation, 21 provinces can be added to the distribution area of $H$. imparilis in Turkey. It is one of the most widespread and abundant species in the country, occurring in 40 provinces, or almost $50 \%$ of all provinces of Turkey. It is found country-wide from sea level up to altitudes of $2000 \mathrm{~m}$, in different types of habitats.

Eryngium billardieri, Eryngium campestre, Melilotus officinalis and Daucus carota are recorded as flowers visited. Adults have quite a long flight period, from mid-May to early September with a peak in July and early August. Probably it has more than one generation a year. The species is frequently recorded in Turkey.

Distribution: Western Palaearctic, common in the Mediterranean basin, Crete, Cyprus, Iran. Turkey: Adana, Amasya, Ankara, Antalya, Bursa, Denizli, Hatay, İstanbul, İzmir, Kastamonu, Kayseri, Konya, Kütahya, Mersin, Nevşehir, Şanlıurfa, Tekirdağ, Trabzon (WARnCKe 1972, as Prosopis brevicornis seducta (FörsTER 1871)).

\section{Hylaeus (Dentigera) intermedius FönSTER, 1871}

Hylaeus intermedius Förster, 1871: 943-944, ơ. Poland: Ratibor. Hylaeus (Dentigera) intermedius Förster, 1871 - DATHE et al. 2016: 16, 44

Material examined: Ankara: Kalecik, 23.05.1990, ơ, leg. S. Risch. Antalya: Arapsuyu, 5 m, 15.10.2003, o, 2 우 오, leg. H. Özbek; 5 m, 16.07.2002, ơ, leg. H. Özbek; 


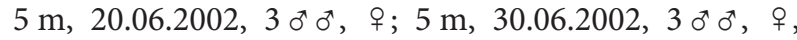
leg. H. Özbek; 10 m, 24.09.2004, ơ , , leg. H. Özbek (on Mentha longifolia). Artvin: Yusufeli, Sarıgöl, 900 m, 16.07.1995, ơ, leg. Gelbrecht/Schwabe. Bingöl: Karlıova, Soğukçeşme, $2000 \mathrm{~m}, 10.08 .2004$, ơ, leg. S. Çoruh (on Centaurea solstitialis). Erzincan: Kemaliye, Avc1, 1250 m, 04.08.2003, ơ, leg. S. Çoruh. Erzurum: Oltu, Başaklı, Karadağ Mt, 2100 m, 24.07.2005, ơ, leg. H. Özbek (on Eryngium creticum). Kars: Sarıkamış, Karakurt, TCK çeşmesi, $1500 \mathrm{~m}, 40^{\circ} 08 \mathrm{~N} 42^{\circ} 21 \mathrm{E}, 19.08 .2003$, o ${ }^{*}$, H. Özbek. Konya: Beyşehir, 700 m, 24.05.1990, o, leg. S. Risch. Muğla: Bodrum, Salmakis, $37^{\circ} 02 \mathrm{~N} 27^{\circ} 25 \mathrm{E}$, 14-25.07.2001, 44 ○ึ ơ, 45 우 우, leg. F. Burger.

Remarks: This species has recently been resurrected as an independent taxon (DATHE et al. 2016). A more recent report for Turkey (without further details) can be found in Schmidt et al. (2015), where DNA barcoding confirms the status of $H$. intermedius as a species; see also Schoder (2018). Ascher \& Pickering (2019) treat $H$. intermedius still as a synonym of $H$. gredleri.

This species is documented hitherto from nine provinces, with its distribution appearing sporadic. It occurs from sea level up to $2100 \mathrm{~m}$. The flight period is from May to October. Some specimens were collected on the flowers of Centaurea solstitialis, Eryngium creticum and Mentha longifolia.

Distribution: Mediterranean and Black Sea countries, Central Europe, Turkey (9 provinces).

\section{Hylaeus (Dentigera) kahri FöRSTER, 1871}

Hylaeus kahri Förster, 1871: 954-956, ơ. Austria.

Hylaeus (Dentigera) kahri Förster, 1871 - Aliev 1986: 263.

DATHE et al. 2016: 16, 45.

Material examined: Adıyaman: 20 km E Gölbaşı, 600 m,

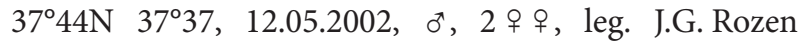
and H. Özbek (coll. AMNH); Karadut, Nemrut Dağ1,

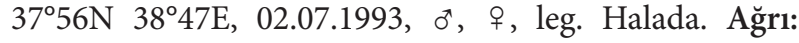

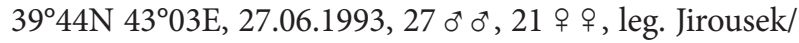
Halada; Eleşkirt 30 km W, 2200 m, 14.07.1987, o, + , leg. R. Hensen. Antalya: Konyaalt1, 3 m, 21.05.2009, 2 ㅇ 우, leg. J.G. Rozen, J.S. Ascher, H. Özbek; Konyaalt1, 5 m,

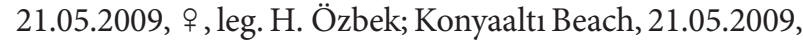
†, leg. Ascher, Rozen \& Özbek (coll. AMNH); Alanya, Demirtaş, 100 m, 29.07.1985, ๆ, leg. R. Hensen; Korkuteli, Kizilcadağ, 1500 m, 03.07.1990, ơ, leg. A.W. Ebmer. Artvin: Yusufei, Kaçkar Mts, Yaylalar, 2200 m, 17.07.1995, $2 \sigma^{\top} \sigma^{*}$, leg. Gelbrecht/Schwarz. Bingöl: Kuruca Geçidi, 08.07.1984, 1800 m, ơ , leg. A.W. Ebmer; Genç, 15 km S, 1400 m, 13.08.1985, ㅇ, leg. R. Hensen. Bitlis: Tatvan, $1750 \mathrm{~m}, 16.08 .1985,2 \sigma^{\star} \sigma^{\star}, 3$ ㅇ 우, leg. R. Hensen; Tatvan, $38^{\circ} 29 \mathrm{~N} 42^{\circ} 17 \mathrm{E}, 30.06 .1983,2 \sigma^{\top} 0^{\star}$, ㅇ, leg. Jirousek/Halada. Burdur: Çeltikçi, 02.09.1983, 5 đ o $\sigma^{\star}$, leg. J. Timmer. Bursa: 300 m, 20.07.1987, ㅇ, leg. R. Hensen; Armutlu, 30 m,
20.08.1985, †, leg. P. van Ooijen. Erzurum: Atatürk University Campus, 1900 m, 13.07.2004, 2 o o o ; 09.08.2004, $\sigma^{7}$, leg. H. Özbek (on Melilotus officinalis); Abdurrahmangazi, 2200 m, 14.07.2000, 2 ㅜ, leg. C. Güçlü; Çat Yolu, Gölet, $2100 \mathrm{~m}, 39^{\circ} 47 \mathrm{~N} 41^{\circ} 09 \mathrm{E}, 09.07 .2004,4 \mathrm{o}^{\star} \mathrm{o}^{\star}$, leg. J.G. Rozen \& H. Özbek; Pasinler, $5 \mathrm{~km} \mathrm{~W}$ of Pasinler, Agricultural Research Station, 1900 m, 01-10.07.2007, 4 o $^{\star} o^{\star}, 2$ ㅇ ㅇ, leg. J.G. Rozen, J.S. Ascher \& H. Özbek (coll. AMNH). Eskişehir: Mihalgazi, Sakırılıca, 06.07.1997, †, leg. Prudek \& Ríha. Gümüşhane: Torul, $40^{\circ} 34 \mathrm{~N}$ $39^{\circ} 17 \mathrm{E}, 1000 \mathrm{~m}, 12.07 .1985,9$ ㅇ 이 leg. M. Schwarz (coll. Schwarz/Ansfelden); SE of Torul, $39^{\circ} 22 \mathrm{E} 40^{\circ} 31 \mathrm{~N}$, 06.09.1970, ơ, leg. Fraser-Jenkins. Hakkâri: Oramar, $10 \mathrm{~km} \mathrm{NE}, 1700 \mathrm{~m}, 29.08 .1985,9$ o $^{\star}$, ㅇ, leg. M. Schwarz (coll. Schwarz/Ansfelden); Yüksekova, 1800 m, 22.7.1988, $80^{\star} o^{\star}, 13$ ㅇ ㅇ , leg. CSchEg; Yüksekova, $15 \mathrm{~km} \mathrm{E,} 2100 \mathrm{~m}$, 20.07.1986, 2 ๙ $^{\top}$, 우, leg. A.W. Ebmer; $20 \mathrm{~km} \mathrm{E,} 2200 \mathrm{~m}$, 23.07.1986, क, leg. A.W. Ebmer; $30 \mathrm{~km} \mathrm{W,} 1850 \mathrm{~m}$, 19.07.1986, $5 o^{\star \top} o^{*}, 2$ 우 우, leg. A.W. Ebmer; Varagöz, Sat

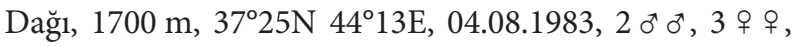
leg. W. Schacht (coll. Schwarz/Ansfelden); Betüşşebap,

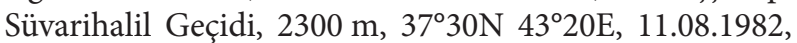
$40^{\star} o^{*}$, + , leg. W. Schacht (coll. Schwarz/Ansfelden); Süvarihalil Geçidi, 2500 m, 27.06.1985, o`, 2 우 ㅇ, leg. M. Schwarz (coll. Schwarz/Ansfelden); Zap Vadisi, Kaval, 1200 m, 22.07.1986, ㅇ, A.W. Ebmer. Hatay: Samandağ 06.07.1996, ơ, leg. Brechtel; 24.07.1998, ð, leg. T. Osten. Dörtyol, Nur Dağları, 06.07.1996, ơ, leg. Brechtel/ Ehrmann. İzmir: Bergama, $300 \mathrm{~m}, 22.07 .1985,2$ 우 우, leg. R. Hensen. Kahramanmaraş: Göksun, 1400 m, 26.06.1987, †, leg. R. Hensen; Salyan, Ahmetcik, 17001800 m, 10.07.1990, o*, leg. A.W. Ebmer; Pürin Geçidi, 1550-1650 m, 10.07.1990, †, leg. A.W. Ebmer. Kars:

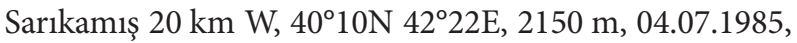
$\mathrm{o}^{7}$, M. Schwarz ; Karakurt, $20 \mathrm{~km} \mathrm{W,} 1600 \mathrm{~m}, 40^{\circ} 10 \mathrm{~N}$ $42^{\circ} 22 \mathrm{E}, 14.07 .1985,{ }^{\star}, 3$ ㅇ ㅇ, leg. M. Schwarz (coll. Schwarz/Ansfelden). Kayseri: Develi, Erciyes, 1800 m, 15.07.1990, ㅇ, leg. A.W. Ebmer; Develi, Bakırdağ, Gezbeli Geçidi, 1750-1900 m, 06.07.1984, 3 o $^{\top} \sigma^{\star}$, 우, leg. A.W. Ebmer; 1700 m, 06.07.1984, ơ, leg. A.W. Ebmer. Konya: Güneysınır, Gürağaç, 1020 m, 06.08.2002, 4 우 우, leg. M. Kesdek; Akşehir, Sultan dağları, 1800 m,07.07.1990, ㅇ, leg. A.W. Ebmer. Kütahya: Sobran, $39^{\circ} 40 \mathrm{~N} 30^{\circ} 10 \mathrm{E}$, 05-08.07.1993, 2 o $^{\star} 0^{\star}, 3$ 우 우, leg. Jirousek (coll. Schwarz/ Ansfelden). Mersin: Silifke, Kizkalesi, 09.05.1988, ơ, leg. N. Mohr; 35 km NNW, Kargican, 200 m, 04.08.1985, ㅇ, leg. R. Hensen; Mut, Sertavul Geçidi,1550 m, 07.07.1990, $20^{\star} o^{\star}$, 우 , leg. A.W. Ebmer. Nevşehir: Kaymaklı, 1200 m, 23.06.1987, 4 우 우, leg. R. Hensen; Avanos, Zelve, 1000 m, 22.06.1987, ơ, 2 우 우, leg. R. Hensen; Zelve, $1050 \mathrm{~m}$, 17.07.1984, 2 , leg. A.W. Ebmer; Ürgüp, Topuzdağ 1 Geçidi, 1300, 13.07.1984, ơ, leg. A.W. Ebmer. Sivas: Gürün, Şuğul Vadisi, 1400 m, 13.07.1990, $40^{\star} \sigma^{\star}$, + , leg. A.W. Ebmer; İmranll, $40 \mathrm{~km} \mathrm{E,} 1700 \mathrm{~m}, 15.07 .1984$, 2 ㅇ 우 , leg. A.W. Ebmer. Van: 1800 m, 13.07.1987, ơ, leg. R. Hensen; town, $38^{\circ} 30 \mathrm{~N} 43^{\circ} 24 \mathrm{E}, 28.06 .1993,80^{\top} 0^{*}$, leg. Halada/Jirousek (coll. Schwarz/Ansfelden); Başkale, $30 \mathrm{~km} \mathrm{~N}, 2700 \mathrm{~m}, 11.07 .1984,4$ ㅇ 아 leg. R. Hensen; 
Güzeldere Geçidi, 2500-2600 m, 09.07.1984, o, 2 우 우, leg. A.W. Ebmer; Achdamar, 1720 m, 17.07.1988, ơ leg. C. Schmid-Egger; Gevaş, Göllü, 3843N 4319E,

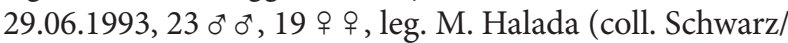
Ansfelden).

Remarks: This is a widespread species found in all regions of the country from sea level to $2700 \mathrm{~m}$ altitude in various habitats. The flight season is from the beginning of May to the end of August. It is frequently recorded from Turkey (23 provinces, Fig. 16.1).

Distribution: Countries bordering the Mediterranean Sea and the Black Sea, from Portugal to Iran. New for Turkey.

\section{Hylaeus (Dentigera) pallidicornis MoraWITz, 1876}

Hylaeus pallidicornis Morawitz, 1876: 290, 우. Uzbekistan: Shakhimardan.

Hylaeus (Dentigera) pallidicornis Morawitz, 1876 - Dathe \& ProshCHALYKIN 2017: 31; 2018: 24.

Material examined: Ağrı: $1650 \mathrm{~m}, 39^{\circ} 44 \mathrm{~N} 43^{\circ} 03 \mathrm{E}$, 27.06.1993, ơ, 8 우 우, leg. Halada/Jirousek (coll. Schwarz/ Ansfelden); Eleşkirt, $30 \mathrm{~km} \mathrm{W,} 2200$ m, 14.07.1987, ㅇ, leg. R. Hensen. Mardin: Midyat, $1100 \mathrm{~m}, 37^{\circ} 25 \mathrm{~N}$ $41^{\circ} 22 \mathrm{E}, 25.05 .1983,2$ 우 우, leg. W. Schacht (coll. Schwarz/ Ansfelden). Nevşehir: Avanos, Zelve, 1000 m, 22.06.1987, ㅇ, leg. R. Hensen; Kaymakl1, 1200 m, 23.06.1987, 운, leg. R. Hensen; Ürgüp, $1100 \mathrm{~m}, 11.08 .1985$, †, leg. P. v. Ooijen. Şanlıurfa: Halfeti, 400 m, 28.06.1987, q, leg. R. Hensen. Van: 1800 m, 13.07.1987, , leg. R. Hensen.

Remarks: This Asian species is new to Turkey. Its distribution is focussed mainly on Eastern and South-Eastern Anatolia, but it has also been recorded from Central Anatolia (Nevşehir). Currently, Nevşehir is next to the Ukraine the westernmost point of its total distribution. $H$. pallidicornis might be considered as a bee species preferring a continental climate at 400-2200 m altitude. The flight period is from the end of May to the middle of August. It is collected only sparsely in Turkey (5 provinces, Fig. 16.2).

Distribution: Central Asia (Kazakhstan, Kyrgyzstan, Tajikistan, Uzbekistan), Mongolia, China, Russia (North Caucasus), Ukraine (Dathe \& Proshchalykin 2018). New to the Turkish fauna.

\section{Hylaeus (Dentigera) punctus (FöRSTER, 1871)}

Hylaeus punctus Förster, 1871: 940-942, ơ ㅇ․ Croatia: Dalmatia.

Prosopis puncta (FöRster, 1871) - WARNCKE 1972.

Hylaeus (Dentigera) punctus (Förster, 1871) - DAthe 1980: 234.
Material examined: Adana: Pozant1, 06.07.1983, + , leg. J. Hladil. Antalya: Alanya, 10 m, 30.08.1983, ㅇ, leg. P. van Ooijen; Kemer W 7 km, Kesme, 130 m, 36 $39 \mathrm{~N}$ 030²9E, 05.06.1998, ơ , leg. S.M. Blank (on Paliurus spina-christ). Burdur: Çeltikçi, $900 \mathrm{~m}, 02.09 .1983$, ơ, leg. J. Timmer. Eskişehir: Sakari, Ilıca, Gümele, 06.07.1997, ㅇ, leg. Prudek/Ríha. Hatay: Samandağ 10 km E, Asi Nehri, 100 m, 05.07.1998, ㅇ, leg. T. Osten; 30 km N, Nur dağları, 06.07.1996, 2 + + ; İskenderun, 30 km S, Güzelyayla, 06.07.1996, $2 \sigma^{\star} \sigma^{\star}$, + , leg. Brechtel/Ehrmann; Belen, 28.08.1983, ơ ${ }^{\star}$, + , leg. J. Timmer. Mersin: Silifke, Uzuncaburç, $400 \mathrm{~m}, 02.08 .1985,2 \sigma^{\star} \sigma^{\star}$, leg. R. Hensen. Muğla: Ölüdeniz, 50 m, 25.07.1986, ㅇ, leg. P. van Ooijen. Nevşehir: Avanoz, 07.07.1983, ơ, leg. J. Hladil. Osmaniye: Yarpuz, 700-1050 m, 12.06.1986, ${ }^{\star}$, leg. M. Bologna. Yalova: Termal, 300 m, 28.08.1985,

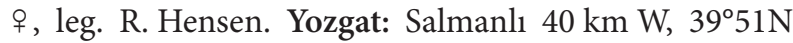
$34^{\circ} 15 \mathrm{E}, 23.05 .2007$, ơ , leg. W.H. Liebig.

Remarks: The provinces Antalya, Burdur, Eskişehir, Mersin, Muğla, Nevşehir, Osmaniye, Yalova and Yozgat have to be added to the hitherto known distribution area. In general, the species is focussed on the Mediterranean and Central Anatolian regions. It can be considered a thermophilic lowland species that occurs mainly below about $1000 \mathrm{~m}$ altitude. The flight period is from the beginning of May to beginning of September. It is collected moderately in Turkey (12 provinces).

Distribution: South-eastern Europe and Middle East including Iran. Turkey: Adana, Bursa, Hatay (WARnCKE 1972).

\section{Hylaeus (Dentigera) rubicola SAUnDERS, 1850}

Hylaeus rubicola SAUnders, 1850: 58, 우․ Greece: Epirus.

Prosopis (Nesoprosopis) rubicola (SAUNDERS, 1850) - WARNCKE 1972: 765.

Hylaeus (Dentigera) rubicola SAUNDERS, 1850 - DATHE 1980: 241.

Material examined: Antalya: Manavgat $10 \mathrm{~km} \mathrm{~W}$, 31.07.1981, 2 ㅇ ㅇ , ơ, leg. R. Hensen. Bursa: Mudanya, $25 \mathrm{~m}, 25.08 .1985$, ㅇ, leg. R. Hensen.

Remarks: H. rubicola was previously known from İzmir and Konya. With the present study, Antalya and Bursa are added to the occurrence area. The distribution shows that it is a thermophilic species of the lowlands, which occurs mainly in the coastal area, but also in Konya (approx. $1000 \mathrm{~m}$ ). It is a less common species with a sporadic occurrence. In Turkey it is collected only sparsely (4 provinces).

Distribution: Eastern Mediterranean, mainly on the Aegean islands. Turkey: İzmir, Konya (WARnCKE 1972). 


\section{Subgenus Hylaeus FaBricIUS, 1793}

\section{Hylaeus (Hylaeus) angustatus (SCHENCK, 1861)}

Prosopis angustata ScHencK, 1861: 321, ơ. Germany: Nassau. Prosopis (Prosopis) angustata angustata SCHENCK, 1859[!] WARNCKE 1972: 752.

Hylaeus (Hylaeus) angustatus (SCHenck, 1861) - Aliev 1986: 266.

Material examined: Antalya: Akseki, Teke Pass N, 1500 m, 06.07.1990, ㅇ, leg. A.W. Ebmer Beydağları, $20 \mathrm{~km}$ S Elmal1, 1600-1700 m, 04.07.1990, 3 우 우, leg. A.W. Ebmer. Artvin: Yusueli, Kaçkar, 1000 m, 16.07.1995, ơ, leg. Gelbrecht/Schwarz. Bingöl: Karlıova, Soğukçeşme, 1415 m, 10.08.2004, o*, leg. S. Çoruh. Erzincan: Refahiye, 1700 m, 15.07.1984, ㅇ, leg. A.W. Ebmer. Erzurum: Köprüköy, Örentaş, 04.07.2004, $2 \sigma^{\star} \sigma^{*}$, leg. H. Özbek (on Cephalaria procera); Ağzıaçık, 2000 m, 07.07.2004, o, J.R. Rozen, H. Özbek (coll. AMNH); Oltu, Tutmaç- Başaklı border, $1900 \mathrm{~m}, 01.07 .2000,3 \sigma^{\star} o^{\star}$, leg. Ö. Çalmaşur; Çamlıbel, $40^{\circ} 2822 \mathrm{~N} \quad 41^{\circ} 4635 \mathrm{E}, 1750 \mathrm{~m}, 11.07 .2004,0^{\star}$, leg. H. Özbek. Hakkâri: SW Yüksekova, Varagöz, 37²5N $44^{\circ} 13 \mathrm{E}, 1700 \mathrm{~m}, 04.08 .1983$, ơ , leg. W. Schacht (coll. Schwarz/Ansfelden). İstanbul: Boğaz (Bosporus), ơ , no other data (coll. RM Stockholm); Karacaköy, $41^{\circ} 09 \mathrm{~N}$ $29^{\circ} 46 \mathrm{E}, 30.07 .1988$, o $^{\star}, 2$ ㅇ 우 leg. Madl; Yeniköy, $41^{\circ} 07 \mathrm{~N} 29^{\circ} 04 \mathrm{E}, 28.07 .1988$, ơ , leg. Madl (coll. Schwarz/ Ansfelden). Kahramanmaraş: Göksun, Prin Pass S site, $1550-1650$ m, 10.07.1990, ㅇ, leg. A.W. Ebmer. Mersin: Mut, Sertavul Pass, 1550 m, 08.07.1990, o`, leg. A.W. Ebmer. Nevşehir: 2 km S, 1250 m, 04.07.1984, $2 o^{*} o^{*}$, leg. A.W. Ebmer; Göreme, $38^{\circ} 39 \mathrm{~N} 34^{\circ} 52 \mathrm{E}$, 17.08.1984, 2 đ̛ $^{\top}$, leg. Schmid-Egger; Göreme, $38^{\circ} 40 \mathrm{~N} 34^{\circ} 50 \mathrm{E}$, 23.06.1993, o ${ }^{*}$, leg. M. Halada (coll. Schwarz/Ansfelden); Avanos, 07.07.1983, o, 우, leg. J. Hladil. Sivas: Yıldızeli, Çamlıbel Pass, 1600-1700 m, 16.07.1984, 2 우 우, leg. A.W. Ebmer.

Remarks: For the first time we can add here distribution data from the East Anatolian region, while new data from the Aegean region are missing. H. angustatus occurs in both warmer and cooler regions, from sea level (İstanbul) to $2000 \mathrm{~m}$ (Erzurum) in various habitats. The flight period is from the end of June to the beginning of August. It is collected moderately in Turkey (14 provinces).

Distribution: Palaearctic. In Turkey previously known from Ankara, İstanbul, Kastamonu, Konya, Kütahya, Mersin (WARNCKE 1972). Now another eight provinces can be added: Antalya, Artvin, Erzincan, Erzurum, Hakkâri, Kahramanmaraş, Nevşehir and Sivas.

\section{Hylaeus (Hylaeus) araxanus (WARNCKE, 1981)}

Prosopis araxana WARncke, 1981: 171, 우 $0^{\star}$. Turkey: Tuzluca, Aras valley; Iran.

Hylaeus (Hylaeus) araxanus (WARNCKE, 1981) - DATHE \& Proshchalykin 2018: 28.

Remarks: Holotype of this species is a male from outermost eastern Turkey (Iğdır), but apart from the this, no other Turkish specimens are known. However, WARNCKE (1981: 192) mentioned 146 specimens of both sexes as paratypes from Iran. Our knowledge about the range of this species was recently extended by DATHE \& ProshCHALYKIN (2018) with evidence from five Central Asian countries. While H. araxanus is widespread and usually has been captured in numbers, it must be classified as rare in Turkey (Fig. 17.2).

This is amazing, because the species is small, but easy to recognize. The wrinkly-striped forehead region of the males is reminiscent of $H$. moricei, but in $H$. araxanus the head is wider than long, the punctation, especially of the mesopleura, coarser, and the sculpture smooth and shiny.

Distribution: Turkey, Iran, Central Asia: Kazakhstan, Uzbekistan, Kyrgyzstan, Turkmenistan, Tajikistan.

\section{Hylaeus (Hylaeus) communis NyLANDER, 1852}

Hylaeus communis Nylander, 1852: 234, 우 $\sigma^{\top}$. France.

Prosopis (Prosopis) communis communis (Nylander, 1852) WARNCKE 1972: 753.

Hylaeus (Hylaeus) communis Nylander, 1852 - Aliev 1986: 266; Proshchalykin \& Dathe 2012: 12.

Material examined: Ankara: Kızılcahamam, 29.07.1987, , leg. J. Wimmer. Antalya: Gündoğmuş, Pembelik, $6 \mathrm{~km} \mathrm{NE}, 36^{\circ} 83 \mathrm{~N} 32^{\circ} 05 \mathrm{E}, 1090 \mathrm{~m}, 02.08 .2009$, o $\mathrm{o}^{\top}$, leg. Schmid-Egger. Artvin: Yusufeli, Altıparmak, 1200-1700 m, 28.08.1995, ○, P. Hartmann; Sarıgöl, Kaçkar dağı, 1000 m, 16.07.1995, o, det. Gelbrecht/ Schwarz; Demirkent, Salekör, 1600 m, 02.09.1995, + , P. Hartmann. Aydın: Nazilli, Beydağ, $250 \mathrm{~m}$, $38^{\circ} 01 \mathrm{~N} 28^{\circ} 18 \mathrm{E}, 24.05 .1981,2 \sigma^{\star} \sigma^{\top}$, leg. Aspöck/Rau./ Res (coll. Mus. Linz). Bitlis: Ahlat, $1750 \mathrm{~m}$, ?, $20^{\top} 0^{\top}$, leg. R. Hensen; Tatvan, $38^{\circ} 29 \mathrm{~N} 42^{\circ} 17 \mathrm{E}, 30.06 .1993$, 3 우, leg. Jirousek/Halada (coll. Schwarz/Ansfelden). Bolu: 18 km W, 18.08.1970, ㅇ, leg. Fraser/ Jenkins; göl kenar1, $40^{\circ} 44 \mathrm{~N} 31^{\circ} 37 \mathrm{E}, 21.06 .1993$, o $0^{\star}$, leg. M. Halada (coll. Schwarz/Ansfelden). Bursa: 225 m, 20.07.1997,

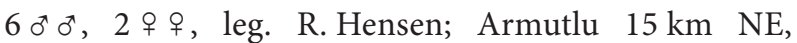
50 m, 27-29.07.1986, 2 o $^{\star}, 4$ 우 ㅇ, leg. P. van Ooijen. Erzincan: Kolçekmezdağı geçidi, 2070 m, 17.07.1996, ơ, leg. P. Rasmont (on Euphorbia sp.). Erzurum: Tortum, Bağbaşı, 20.09.1977, female, leg. H. Özbek (on Trifolium pratense L.); Pasinler, $5 \mathrm{~km} \mathrm{~W}$ of Pasinler, Agricultural Research Station, 10.07.2007, क, leg. J.S. Ascher, 
H. Özbek, J.G. Rozen (coll. AMNH); Atatürk University

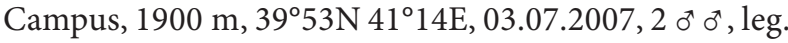
J.S. Ascher, H. Özbek, J.G. Rozen (coll. AMNH); Uzundere, Karadağ, 2250 m, 02.09.1995, ㅇ, leg. M. Kraus (on Euphorbia sp.). Hakkâri: Beytüşşebap, 1400 m, 06.07.1987, 우, R. Hensen. Kars: Kağ1zman 14 km E, 1700 m, 24.07.1986, ơ, leg. A.W. Ebmer. Konya: Alaaddin Hill, 1050 m, 20.06.1987, 2 ㅇ ㅇ, leg. R. Hensen; Beyşehir, 1150 m, 18.06.1987, o , leg. R. Hensen. Manisa: Akçakertik beli, $39^{\circ} 07 \mathrm{~N} 28^{\circ} 42 \mathrm{E}, 800 \mathrm{~m}, 17.05 .1983$, ㅇ, Rausch/Ressl (coll. Mus. Linz). Nevşehir: $2 \mathrm{~km} \mathrm{~S}$, 1250 m, 04.07.1984, 우, leg. A.W. Ebmer. Niğde: Çiftehan, Bolkar dağları, 1700-1800 m, 17.07.1990, ơ, leg. A.W. Ebmer. Rize: $40 \mathrm{~km} \mathrm{~N}$ of İspir, ca $40^{\circ} 50 \mathrm{~N} 40^{\circ} 40 \mathrm{E}$, 1400 m, 11.07.1985, ㅇ, leg. M. Schwarz (coll. Schwarz/ Ansfelden). Van: 1800 m, 13.07.1987, 2 o $^{\star}, 7$ 우 우, leg. R. Hensen. Yalova: Termal, $300 \mathrm{~m}, 28.08 .1985,0^{\top}$, 우, leg. R. Hensen.

Remarks: H. communis is the most common species in the genus, at least in Europe, but also with a wide distribution beyond that. It has even been introduced to the American continent. As a result of the wide distribution, various morphological variants have been described, but these have not yet been studied in detail throughout the entire range.

The flight period is June to the end of September. $H$. communis was collected in all regions of the country, with the exception of south-east Anatolia. Our compilation adds 15 provinces to the distribution data. It is frequently recorded in Turkey (25 provinces).

Distribution: Throughout Europa, North Africa, Central and Northern Asia (Dathe \& Proshchalykin 2018); Turkey: Amasya, Ankara, Bolu, Bursa, İzmir, Kocaeli, Kütahya, Mersin, Sinop (WARncke 1972); Erzurum (Özbeк 1977); Antalya, Artvin, Aydın, Bitlis, Erzincan, Erzurum, Hakkâri, Kars, Konya, Manisa, Nevşehir, Niğde, Rize, Van, Yalova.

\section{Hylaeus (Hylaeus) crispulus Dathe, 1980}

Hylaeus (Hylaeus) crispulus DAthe, 1980: 82, 84-85, 87, ơ + . Iran: Elburs.

Prosopis hermona WARNCKE, 1981: 181-182, क o $0^{\star}$. Israel: Mt. Hermon.

Prosopis hermona anatolica WARNCKE, 1981: 182, $0^{\top}$ ㅇ. Turkey: Gürün.

Prosopis (Prosopis) crispula hermona WARNCKE, 1981 WARnCKe 1992: 792.

Prosopis (Prosopis) crispula anatolica WARNCKE, 1981 WARNCKE 1992: 792.

Material examined: Adıyaman: Kâhta, Nemrut Mt, 2100 m, 11.07.1990, o, leg. A.W. Ebmer. Konya: Akşehir, Sultan Dağları, NE, 1800 m, 18.07.1990, 우 leg. A.W. Ebmer. Malatya: Darende, 7 km W, 1400 m,
13.07.1990, ơ , leg. A.W. Ebmer. Sivas: Gürün, NW Şuğul, 1400 m, 13.07.1990, o, 31.07.1986, o* , + , leg. A.W. Ebmer.

Remarks: The species is well characterized in the male by a strong transverse impression in the mask directly below the antenna bases; besides these there is a shiny black pit on the orbit margin, so that the side spots appear constricted. The antennae are slender; the general body habit is rather robust and compact, with mostly distinct hairiness. The copulatory apparatus of the holotype is missing due to inappropriate preparation by Warncke.

The taxon is known from northern Iran (Elburs), Israel and Turkey. WARNCKe (1992: 792) himself synonymized the forms he described as Prosopis hermona and $P$. hermona anatolica from Israel and Turkey respectively. In our opinion the geographical differences do not justify a subspecies status. It is moderately recorded from Turkey (11 provinces).

Distribution: Israel, Iran. Turkey: Sivas, Hakkâri (2600-3000 m) (WARNCKE 1981, as Prosopis hermona anatolica), Bitlis, Erzincan, Gümüşhane, Hakkâri, Karaman, Kars, Konya, Niğde (WARncke 1992, as P. crispula anatolica); Adiyaman, Malatya and Sivas provinces are added here.

\section{Hylaeus (Hylaeus) deceptorius (BENOIST, 1959)}

Prosopis deceptoria Benoist, 1959: 77, 86, ㅇ o ${ }^{\star}$. France: Corsica. Prosopis communis deceptoria BenoIst, 1959 - WARNCKE 1972: 754.

Hylaeus (Hylaeus) deceptorius (Benoist, 1959) - DATHE 2000: 166.

Material examined. Ardahan: Göle, Karınca Düzü, 2160 m, 25.07.2005, 2 ơ o leg. C. Güçlü (on Eryngium sp.). Bingöl: Karlıova, Çobançantası, 1455 m, 10.08.2004, ơ , leg. S. Çoruh; Soğukçeşme, $1455 \mathrm{~m}, 10.08 .2004,20^{\star} \mathrm{o}^{\star}$, 5 우 우, leg. S. Çoruh (on Centaurea solstitialis). Erzurum: Narman, Kireçli Dağı, 2000 m, 17.08.2004, 2 o o leg. S. Çoruh; Oltu, Subatık, 1300 m, 13.08.2004, 2 우 이 leg. H. Özbek (on Melilotus alba); Tortum, Derekap1, 1000 m, 20.09.2000, 2 우 우, leg. H. Özbek; Tortum, Kazandere, $1200 \mathrm{~m}, 03.08 .2004,2 \sigma^{\star} \sigma^{\star}, 2$ ㅇ ㅇ , leg. S. Çoruh; $20 \mathrm{~km} \mathrm{SE}$ of Tortum, $2100 \mathrm{~m}, 21.07 .2010$, ơ , leg. J.S. Ascher, H. Özbek, J.G. Rozen. Konya: Güneysınır, Gürağaç, 1150 m, 16.07.2000, o, ㅇ leg. M. Kesdek.

Remarks: $H$. deceptorius is originally described as a West Palaearctic species with occurrences on the large Mediterranean islands of Corsica, Sardinia, Sicily and Crete, as well as in Morocco and Greece. The new reports of this species from Turkey with a focus on the East Anatolian region - where Ardahan is the easternmost point - are not unproblematic. Also their occurrence at higher alti- 
tudes of 1000-2200 $\mathrm{m}$ does not fit well to the general behaviour of the species. Further investigations would be very welcome.

DAthe (2013) noted H. deceptorius as a threatened species, whereas in eastern Anatolia it is quite numerous. Plant association: Centaurea solstitialis, Eryngium sp., Melilotus alba. It is a sparsely recorded species in Turkey (4 provinces, Fig. 16.3).

Distribution: Corsica, Crete, Greece, Sardinia, Sicily, Morocco (Ascher \& Pickering 2019). New to Turkey: Ardahan, Bingöl, Erzurum, Konya.

\section{Hylaeus (Hylaeus) dolichocephalus MorawITz, 1876}

Hylaeus dolichocephalus Morawitz, 1876:271-272, 웅. Turkestan: Ferghana.

Prosopis heliaca WARncke, 1992: 791, 웅. Turkey: Bitlis.

Prosopis (Prosopis) dolichocephala (Morawitz, 1876) WARNCKE 1992.

Hylaeus (Hylaeus) dolichocephalus Morawitz, 1876 - Dathe \& Proshchalykin 2017: 17-18.

Material examined: Kayseri: Erciyes Mt, 1850, 05.07.1984, ơ, leg. A.W. Ebmer. Sivas: Gürün, Ziyaret Pass, 2000 m, 15.07.1986, ơ , ㅇ, leg. A.W. Ebmer. Van:

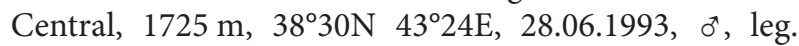
Jirousek (coll. Schwarz/Ansfelden).

Remarks: H. dolichocephalus is a characteristic species of Asian mountains, occurring at 1400-3000 m altitude. It can hardly be misidentified, because of its elongated head shape. Sivas is added to the distribution records; the record from the province Kayseri is the westernmost detected point of distribution. It is moderately collected in Turkey (8 provinces).

Distribution: Central Asia, Afghanistan, Mongolia, Russia, China and Turkey (Dathe \& Proshchalykin 2018). In Turkey: Bitlis, Erzincan, Erzurum, Hakkâri, Kars, Kayseri, Van (Warncke 1992, as Prosopis heliacus); Sivas.

\section{Hylaeus (Hylaeus) funereus (WARNCKE, 1992)} Fig. 4

Prosopis (Nesoprosopis) funerea WARNCKE, 1992: 785-786, 우 $0^{7}$. Turkey: Hakkâri.

Hylaeus (Hylaeus) funereus (WARNCKE, 1992) - ITIS (2020).

Remarks: A small, dainty species, reminiscent of $H$. trifidus in the shape of the mask: the tip of the lateral marks is at the level of the antenna bases; in the upper part they are separated from the compound eye margin by an impressed shiny surface. In $H$. trifidus this surface is oval and clearly defined, in H. funereus there is a blurred furrow extended upward along the orbits. The apical process of sternum 8 is conoid with lateral bristles, in $H$. trifidus with expanded lobus with $\mathrm{V}$-shaped notch; in this structure $H$. funereus resembles more H. sidensis. The propodeum is rounded and without ringlets.

$H$. funereus was described from Hakkâri and Adıyaman (1200-2500 m). Unfortunately no additional material has been collected since then. The available data show that $H$. funereus is restricted to the south-eastern part of the country. It is rarely recorded from Turkey (2 provinces, Fig. 17.VII).

Distribution: Turkey: Hakkâri, Adıyaman.

\section{Hylaeus (Hylaeus) gracilicornis (MoraWITz, 1867)}

Prosopis gracilicornis Morawitz, 1867:56-58, 우. Switzerland: St. Moritz.

Hylaeus (Hylaeus) gracilicornis (Morawitz, 1867) Proshchalykin \& Dathe 2012: 14.

Material examined: Hakkâri: Yüksekova, Varagöz, $1650 \mathrm{~m}, 37^{\circ} 25 \mathrm{~N} 44^{\circ} 13 \mathrm{E}, 26.06 .1985$, o $^{\star}, 2$ 우 우, leg. C.J. Zwakhals.

Remarks: Although H. gracilicornis is a widespread species in Europe and Asia, in Turkey it is currently only recorded from Hakkâri. It is rarely found in Turkey (1 province, Figs 16.4, 17.3). New to Turkey.

Distribution: Transpalaearctic species, from France to Russian Far East. In Turkey: Hakkâri.

\section{Hylaeus (Hylaeus) implicatus DATHE, 1980}

Hylaeus (Hylaeus) implicatus DATHE, 1980: 88, 91-92 ơ. Iran: Zoshk near Mandiz.

Prosopis (Prosopis) communis implicata (DATHe, 1980) WARNCKE 1992: 791.

Material examined: Aksaray: Ihlara, $1100 \mathrm{~m}, 38^{\circ} 14 \mathrm{~N}$ 34018E, 18.07.1998, ơ , leg. C. Schmid-Egger. Hakkâri:

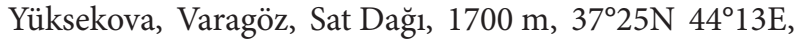

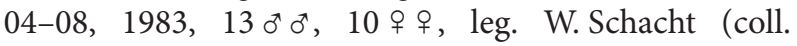
Schwarz/Ansfelden). Nevşehir: Göreme, 1150 m, 38³9N

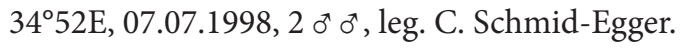

Remarks: H. implicatus was originally described from Iran, later by WARNCKE (1992) from Hakkâri. Here the provinces Aksaray and Nevşehir are added to the distribution data. The species is found only sparsely in Turkey (3 provinces).

Distribution: Iran; Turkey: Aksaray, Nevşehir, Hakkâri. 


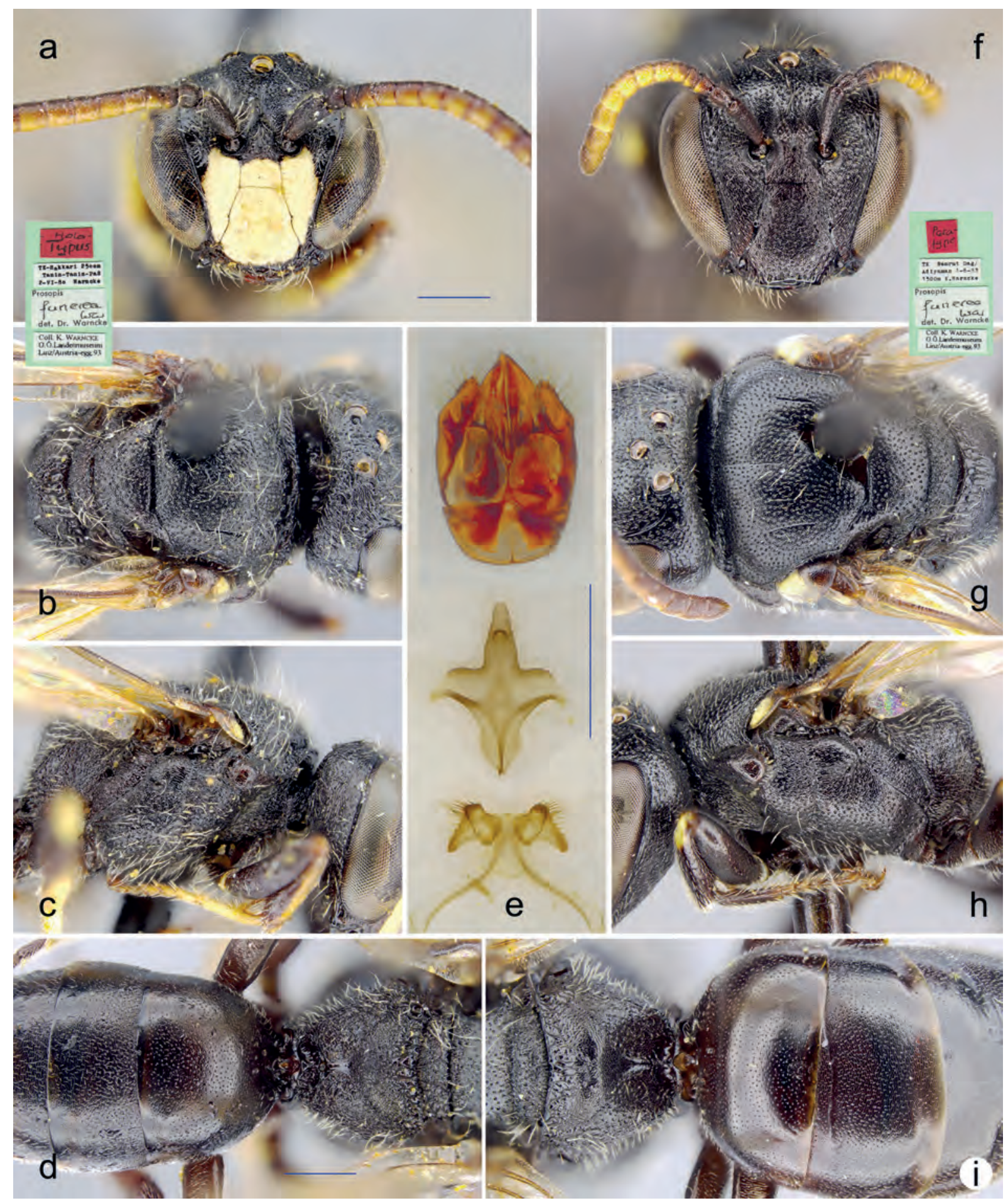

Fig. 4: H. (Hylaeus) funereus (WARNCKe, 1992). Holotype male: a-face, b-mesonotum, c-mesopleuron, d-propodeum and metasoma, e-terminalia. Paratype female: $\mathbf{f}$-face, $\mathbf{g}$-mesonotum, $\mathbf{h}$-mesopleuron, $\mathbf{i}$-propodeum and metasoma. - Scale bar $0.5 \mathrm{~mm}$.

Hylaeus (Hylaeus) jantaris DATHE, 1980

Hylaeus (Hylaeus) jantaris DATHE, 1980: 87, 89-90, ơ ․ . Iran: Elburs.

Prosopis (Prosopis) querquedula WARNCKE, 1981: 178-180, ๙ $\circ$. Turkey: Hakkâri.

Prosopis (Prosopis) jantaris (DATHE, 1980) - WARNCKE 1992: 769.

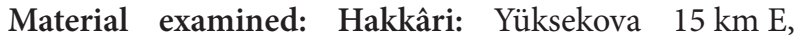
2100-2200 m, 20.07.1986, $5 \sigma^{\star \top} \sigma^{*}, 4$ ㅇ ㅇ, leg. A.W. Ebmer; Yüksekova $20 \mathrm{~km} \mathrm{E,} 2200 \mathrm{~m}, 23.07 .1986,2 o^{\star} o^{\star}, 2$ ㅇ ㅇ , leg. A.W. Ebmer. Van: Başkale 30 km N, 2700 m, 11.7.1987, $\sigma^{\star}$, 우, leg. R. Hensen; Hoşap, Güzeldere Geçidi, 25002600 m, 09.07.1984, ơ, leg. A.W. Ebmer; 2600-2800 m, 18.07.1986, ơ , leg. A.W. Ebmer. 
Remarks: The province Van has been added to the distribution list of $H$. jantaris. The available data show that the occurrence of this species is limited to the eastern part of eastern Anatolia. It is a high mountain species living at an altitude of $2100-3000 \mathrm{~m}$. It was rarely collected in Turkey (2 provinces, Fig. 17.III). In order to specify the distribution data of this species exactly, further investigations should be carried out, especially on the high mountains of the country.

Distribution: Iran, Turkey. In Turkey: Hakkâri (WARNCKE 1981, as P. querquedula), Van.

\section{Hylaeus (Hylaeus) kotschisus (WARNCKE, 1981)}

Prosopis nigrita kotschisa WARNCKE, 1981: 174, † ơ. Turkey: Şereflikoçhisar.

Prosopis (Prosopis) nigrita kotschisa WARNCKE, 1981 - WARNCKE 1992: 763.

Hylaeus (Hylaeus) kotschisus (WARNCKe, 1981) - DATHE 1980: 92.

Material examined: Adıyaman: Kahda, Nemrut Dağ 1 , $2100 \mathrm{~m}, 11.07 .1990,3 \sigma^{\star} \sigma^{*}$, leg. A.W. Ebmer. Ağri: Hamur, 1650 m, 26.07.2003, $2 \sigma^{\top} \sigma^{\star}$, ㅇ , leg. H. Özbek. Artvin: Yusufeli, $850 \mathrm{~m}, 27.07 .2004,30^{\top} o^{\top}$, leg. S. Çoruh; Demirkent, Salekör, 1650 m, 01.09.1995, 오 leg. M. Kraus; Aksaray: Ihlara, $38.14 \mathrm{~N} 34.18 \mathrm{E}$, 18.07.1998, ơ, leg. C. Schmid-Egger. Bitlis: Ahlat, $1750 \mathrm{~m}, 15.08 .1985,3 \sigma^{\star} o^{\star}$, leg. R. Hensen. Erzurum:

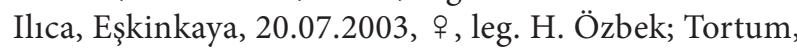
Derekap1, 20.09.2000, ㅇ, leg. H. Özbek (on Melilotus alba); Uzundere, Denizbaşı, 4 ㅇ 오, leg. H. Özbek (on Daucus carota); Hakkâri; 1750 m, 09.07.1987, 3 o $^{\text {đ }}$, 2 우 우, leg. R. Hensen; Yüksekova, $30 \mathrm{~km} \mathrm{W,} 1850 \mathrm{~m}$, 19.07.1986, ơ, leg. A.W. Ebmer; Varagöz, 37²5N $44^{\circ} 13 \mathrm{E}, 1650 \mathrm{~m}, 02.08 .1986,2 o^{\star} o^{\star}, 2$ ㅇ , leg. S.M. Blank; Sat Dağ $1,1700 \mathrm{~m}, 37^{\circ} 25 \mathrm{~N} 44^{\circ} 13 \mathrm{E}, 04.08 .1983$, ơ , leg. M. Schwarz (coll. Schwarz/Ansfelden). Kars: Sarıkamış, Karakurt, TCK Çeşmesi, $1501 \mathrm{~m}, 40^{\circ} 08 \mathrm{~N} 42^{\circ} 21 \mathrm{E}$, 14.08.2007, $6 \mathrm{o}^{\star} \mathrm{o}^{\star}$, leg. H. Özbek (on Eryngium campestre). Kayseri: Pınarbaşı, 1500 m, 25.06.1987, ơ , leg. R. Hensen. Mersin: Silifke, Köselerli, $36^{\circ} 33 \mathrm{~N} 33^{\circ} 27 \mathrm{E}$, 19.07.1988, ơ, leg. C. Schmid-Egger. Nevşehir: 2 km S, 1250 m, 04.07.1984, †, leg, A.W. Ebmer; Avanos, Zelve, 1050 m, 18.07.1984, ơ, leg. A.W. Ebmer. Ürgüp, 1200 m, 24.06.1987, leg. R. Hensen; 1100, 11.08.1987, $2 \sigma^{x} o^{x}$, leg. P. van Ojen. Niğde: Çiftehan, Bolkardağları, 1700-1800 m, 17.07.1990, 2 우, leg. A.W. Ebmer. Sivas: Gürün, Şuğul Vadisi, 1400 m, 31.07.1987, o*, 2 우 온 leg. A.W. Ebmer. Tunceli: Ovacik $17 \mathrm{~km} \mathrm{~W}$, $1250 \mathrm{~m}, 18.08 .1985,2 \sigma^{\star} \sigma^{\star}, 4$ 우 우, leg. R. Hensen. Van: Adilcevaz, 1800 m, 11.07.1984, ơ, leg. A.W. Ebmer. Başkale, Güzeldere Geçidi, 2100 m, 12.07.1984, ㅇ, leg. A.W. Ebmer.
Remarks: The provinces Adryaman, Ağrı, Artvin, Aksaray, Bitlis, Kayseri, Nevşehir, Niğde, Sivas, Tunceli and Van are to be added to the distribution list of H. kotschisus. The species has also been reported from Ankara, but it is mainly found in the eastern parts of Turkey. Ankara is currently the most western distribution point of this species. It can be considered as an inhabitant of warm, dry, steppe-like areas as well as a mountain species. $H$. kotschisus is frequently recorded in Turkey (18 provinces).

Distribution: Eastern Mediterranean species (Lebanon, Iran, Israel, Turkey). In Turkey: Ankara, Erzurum, Hakkâri, Kars, Kirıkkale, Konya, Tokat (WARnCKE 1981); Adıyaman, Ağrı, Artvin, Aksaray, Bitlis, Kayseri, Nevşehir, Niğde, Sivas, Tunceli, Van.

\section{Hylaeus (Hylaeus) kurdus (WARNCKE, 1981) \\ Fig. 5}

Prosopis kurda WARNCKe, 1981:182-183, ơ $\odot$. Turkey: Hakkâri.

Prosopis (Prosopis) kurda WARNCKe, 1981 - WARNCKe 1992: 769, 792.

Hylaeus (Hylaeus) kurdus (WARNCKE, 1981) - ITIS (2020).

Material examined: Van: Gürpınar, Hoşap, Güzeldere Geçidi, 2500-2600 m, 09-12.07.1984, 4 o $^{\star}$ o $^{\pi}, 2$ 우 우, leg. A.W. Ebmer.

Remarks: In the males of this species, the mask is transversely impressed in a bow below the antennal bases. The scapes are black, basally curved and distinctly enlarged over half of their length; apically they are flattened. The lateral marks run along the orbits, are rounded at the top and narrowly edged; at this point there are also smooth surfaces. These characters clearly distinguish this species from the similar $H$. trochilus (see there). The characteristic "rounded versus edged posterior margin of the vertex" (WARNCKE 1992: 792) is incomprehensible as a difference between $H$. kurdus and $H$. crispulus.

H. kurdus was described from Hakkâri. Later it was also recorded in Bitlis and various additional localities in Hakkâri. Here, Van is added. Current and previous records show that it is a high mountain species, occurring up to $3100 \mathrm{~m}$, mainly restricted to the eastern Anatolian region. It is only sparsely collected in Turkey (3 provinces).

Distribution: H. kurdus is according to present knowledge a purely Turkish species. Provinces: Hakkâri (type locality, 2600-3000 m), Bitlis (2500-3100 m) (WARNCKE 1992); Van (2500-2600 m). 


\section{Hylaeus (Hylaeus) leptocephalus (MorawITz, 1871)}

Prosopis leptocephala Morawitz 1871:324-325, ㅇำ. Russia: Saratov.

Hylaeus bisinuatus Förster, 1871: 1048-1050, ơ. South France. Prosopis (Prosopis) bisinuata (FöRster, 1871) - WARNCKE 1972: 753; 1981: 191.

Hylaeus (Hylaeus) bisinuatus Förster, 1871 - Aliev 1986: 266. Prosopis (Prosopis) leptocephala MoRawitz 1870[!] - WARNCKE 1992: 769.

Hylaeus (Hylaeus) leptocephalus (Morawitz, 1871) - ProshCHALYKIN \& DATHE 2012: 14; 2017: 61.

Material examined: Ağrı: Patnos, 22.08.1983, ㅇ, leg. Ooijen. Aksaray: Koçaş, 20 km NE, 900 m, 03.07.1984, $\mathrm{o}^{*}$, ㅇ, leg. A.W. Ebmer; Yeşilova 15 W, 900 m, 03.07.1984, $\sigma^{*}$, + , leg. A.W. Ebmer. Antalya: Alanya, İncekum, $20 \mathrm{~m}, 07.08 .1985$, ơ, leg. P. van Ooijen; Termessos, 1150 m, 16.07.1985, ơ , leg. O. Niehuis. Aydın: Kuşadası, 08.05.1992, $2 \sigma^{\top} \sigma^{\star}$, leg. W.H. Liebig. Balıkesir: Burhaniye, Üren, 10 m, 18.07.1985, ơ, leg. P. van Ooijen. Bayburt: Kop Dağ ${ }_{1}$ Geçidi, 2400 m, 25.07.2010, o , leg. J.S. Ascher, H. Özbek, J.G. Rozen. Bingöl: Karlıova, Soğukceşme, 1040 m, 16.08.2005, 3 o $^{\star}$, 2 ㅇ ㅇ , leg. H. Özbek (on Centaurea solstitialis). Bitlis: Ahlat, $1750 \mathrm{~m}, 15.08 .1985,2 \sigma^{\star} \sigma^{*}$, 우, R. Hensen; Asağı Kolbaşı, 1700 m, 22.07.2003, 2 o $^{\star}$, leg. H. Özbek. Burdur: Çeltikçi, 02.09.1984, o , leg. J. Timmer. Bursa: $225 \mathrm{~m}, 24.08 .1985,7 \sigma^{\star} \sigma^{\star}$, + , leg. R. Hensen; Mudanya, 25 m, 25.08.1985, ơ, R. Hensen. Diyarbakır: 18.07.1977, 2 우 우, leg. J. Timmer; $660 \mathrm{~m}, 14.07 .1986, \sigma^{\star}$, ㅇ, leg. P v Oojen; $650 \mathrm{~m}, 14.07 .1986,09.08 .1985,10 \sigma^{\top} \sigma^{\top}$, ㅇ, leg. R. Hensen. Erzincan: 07.07.2001, $2 \sigma^{\star} o^{\star}$, leg. J.G. Rozen: Erzurum: Atatürk Unersity Campus, 2000 m, 09.08.2004, ơ, ㅇ; 17.08.2003, 3 우, leg. H. Özbek (on Melilotus alba); İspir, $1400 \mathrm{~m}, 29.08 .2003,2 \mathrm{o}^{\star} \mathrm{o}^{\star}$, leg. H. Özbek; $14 \mathrm{~km}$ ENE of Pasinler, 04.07.2007, ㅇ, leg. J.S. Ascher, H. Özbek, J.G. Rozen (coll. AMNH); Oltu, 4 km WSW, 09.07.2007, 2 o $^{\star}$, leg. J. Rozen, J.S. Ascher, H. Özbek (coll. AMNH); Başaklı, $1600 \mathrm{~m}, 29^{\circ} 14 \mathrm{~N} 41^{\circ} 48 \mathrm{E}$, 03.07.2001, 5 ㅇ ㅇ , leg. J.G. Rozen, H. Özbek (coll. AMNH); Çamlibel, $22 \mathrm{~km}$ SW Oltu, 03-08.07.2004, 7 o o o , 4 우 우, leg. J. Rozen, H. Özbek (coll. AMNH); Hınıs, Akören, 19.07.2003, đ`, leg. J. Rozen, H. Özbek (coll. AMNH). Iğdır: 19.8.1983, 5 o $^{\star}$, leg. J. Timmer; Aralık, 900 m, 27.07.2000, , C. Güçlü (on Daucus carota L.); Gaziler, $1040 \mathrm{~m}, 16.08 .2005,2$ 우 우, leg. H. Özbek (on Melilotus alba). Kahramanmaraş: Göksun, 1400 m, 26.06.1987, ${ }^{*}$, leg. R. Hensen. Kars: Sarıkamış, Karakurt, TCK Çesmesi, $1500 \mathrm{~m}, 40^{\circ} 08 \mathrm{~N} 42^{\circ} 21 \mathrm{E}, 19.08 .2003$, o*, 우 , leg. S. Çoruh. Kayseri: Pınarbaşı, 1500 m, 25.06.1987, ơ , leg. R. Hensen. Konya: Alaaddin tepesi,1050 m, 20.06.1987, $\sigma^{\star}$, leg. R. Hensen; Beyşehir, $1150 \mathrm{~m}, 18.06 .1987,6 \sigma^{\star} \sigma^{*}$, leg. R. Hensen; Çumra, $1017 \mathrm{~m}, 13.08 .2000,2 \sigma^{*} \sigma^{*}$, leg. M. Kesdek (Daucus carota). Mersin: Mut $10 \mathrm{~km} \mathrm{S,}$ 08.06.1966, ơ, leg. HHF. Hamann; Dereköy, 02.09.1987, $\sigma^{\star}$, leg. H. Özbek (on Vitex agnus-castus); Silifke, Korikos, $10 \mathrm{~m}$, 03.08.1985, ㅇ, leg. R. Hensen (on Polygonum). Muğla: Bodrum, Salkamış, 13-24.07.2001, $37^{\circ} 02 \mathrm{~N}$ $27^{\circ} 25 \mathrm{E}, 3$ o $^{\top} \mathrm{o}^{\star}$, leg. F. Burger. Nevşehir: $2 \mathrm{~km} \mathrm{~S}, 1250 \mathrm{~m}$, 09.07.1984, ơ , leg. A.W. Ebmer; Göreme, $38^{\circ} 40 \mathrm{~N} 34^{\circ} 50 \mathrm{E}$, 23.06.1993, 우, leg. M. Halada (coll. Schwarz/Ansfelden); Ürgüp, $1100 \mathrm{~m}, 11.08 .1985,2 \sigma^{\star} \sigma^{\star}$, + , leg. P. v. Ooijen. Şanlıurfa: Birecik, 400 m, 06.08.1985, o $\sigma^{*}$, leg. R. Hensen; Halfeti, 425 m, 07.08.1985, 11 o $^{\star}$, ㅇ, leg. R. Hensen; $400 \mathrm{~m}, 28.06 .1987,3 \mathrm{o}^{\star} \mathrm{o}^{\star}$, R. Hensen; Suruç, $400 \mathrm{~m}$, 29.06.1987, o, 3 우 ㅇ, leg. R. Hensen. Tunçeli: Ovacık, $17 \mathrm{~km}$ W, $1250 \mathrm{~m}, 19.08 .1985,2$ o $^{\star}$, leg. R. Hensen. Van: $1800 \mathrm{~m}, 13.07 .1987$, ơ , leg. R. Hensen; Erciş, $1750 \mathrm{~m}$, $38^{\circ} 43 \mathrm{~N} 43^{\circ} 19 \mathrm{E}$, + , leg. W. Schacht (coll. Schwarz/ Ansfelden). Yalova: Çiftlik Köyü, 50 m, 31.07.1986, $2 \sigma^{\star} \sigma^{\star}$, leg. P. van Ooijen.

Remarks: Twenty one provinces are added here to the range of $H$. leptocephalus. Earlier and current records show that this species occurs in various biotopes throughout Turkey, from sea level up to $2400 \mathrm{~m}$ altitude. It is found especially in open areas. Interestingly, it has not been recorded in Hakkâri. Flowers visited: Centaurea solstitialis, Daucus carota, Melilotus alba, Vitex agnuscastus. The flight season is quite long, from early May to early September, with a peak in July; possibly the species has more than one generation per year. It is frequently recorded in Turkey (31 provinces).

Distribution: Throughout Europe, Russia to Far East, Central Asia, Armenia, Azerbaijan, Iran; introduced to North America. Turkey: provinces Adana, Ankara, Aydın, Bursa, Denizli, Kırıkkale, Konya, Kütahya, Sakarya (WARNCKe 1972); Erzurum (ÖzBEK 1977) (all as H. bisinuatus Förster, 1871); Ağr1, Aksaray, Antalya, Balıkesir, Bayburt, Bingöl, Bitlis, Burdur, Diyarbakır, Erzincan, Iğdır, Kahramanmaraş, Kars, Kayseri, Mersin, Muğla, Nevşehir, Şanlıurfa, Tunçeli, Van, Yalova.

\section{Hylaeus (Hylaeus) monedulus (WARNCKE, 1992) Fig. 6}

Prosopis (Prosopis) monedula WARncke, 1992: 790, 801, i o ${ }^{7}$. Turkey: Nemrut Dag.

Hylaeus (Hylaeus) monedulus (WARNCKe, 1992) - ITIS (2020).

Remarks: It is a small, hardly known species. Its sculpture is generally smooth with strong punctation. H. fedtschenkoi resembles it most of all, but is less shiny. Furthermore, the male's scape is shorter, the mask with distinct and deep punctation. In both sexes the antennae are short, the outline of the head is broadly trapezoidal. The species is not comparable with $H$. communis, at most with $H$. piotris Dathe \& Proshchalykin, 2018, which appears slimmer in habitus, face and scapes.

Distribution: H. monedulus was described from Bitlis (Nemrut Mt, $2000 \mathrm{~m}$ ) as indigenous in Turkey, represented by only two specimens ( $\sigma$ and $\circ$, Fig. 17.4). No new material was available for the present study. 


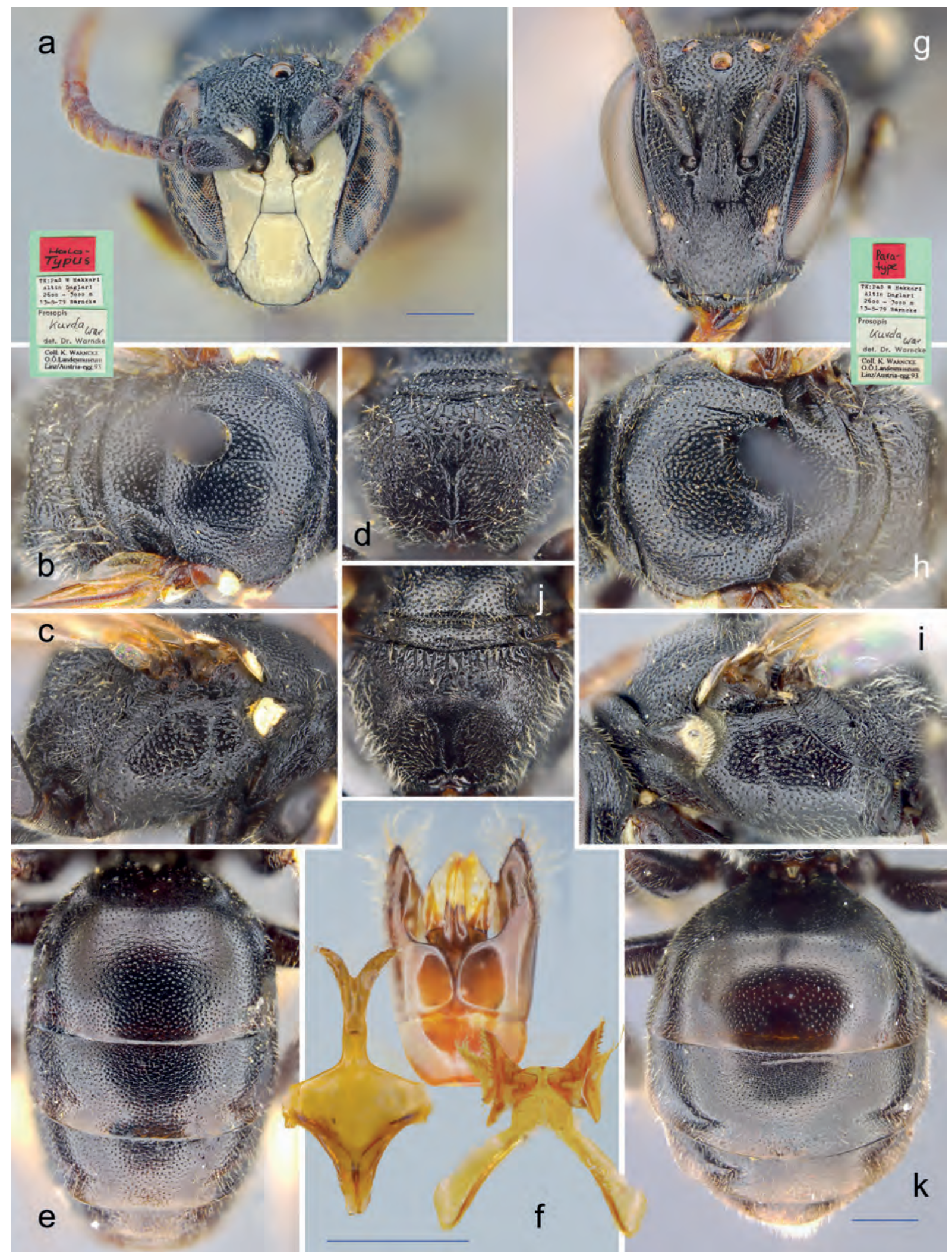

Fig. 5: H. (Hylaeus) kurdus (WARncke, 1981). Holotype male: a-face, b-mesonotum, c-mesopleuron, d-propodeum, e-metasoma, f-terminalia. Paratype female: $\mathbf{g}$-face, $\mathbf{h}$-mesonotum, $\mathbf{i}$-mesopleuron, $\mathbf{j}$-propodeum, $\mathbf{k}$-metasoma. - Scale bar $0.5 \mathrm{~mm}$. 


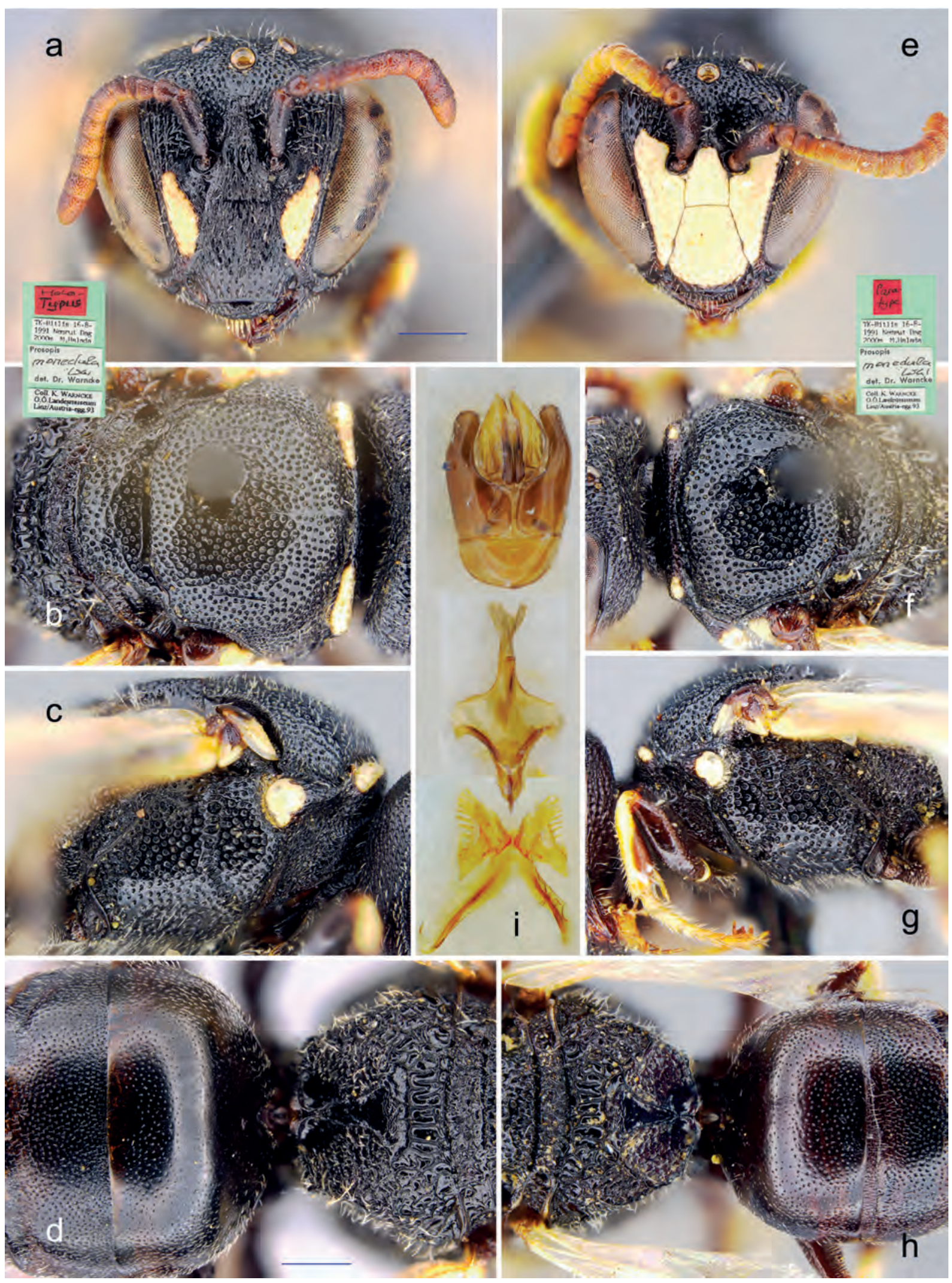

Fig. 6: H. (Hylaeus) monedulus (WARnCKE, 1992). Holotype female: a-face, b-mesonotum, c-mesopleuron, d-propodeum and metasoma. Paratype male: e-face, $\mathbf{f}$-mesonotum, $\mathbf{g}$-mesopleuron, $\mathbf{h}$-propodeum and metasoma, $\mathbf{i}$-terminalia. - Scale bar $0.5 \mathrm{~mm}$. 


\section{Hylaeus (Hylaeus) moricei (FrIESE, 1898)}

Prosopis moricei FrIeSe, 1898: 310, ㅇ ơ. Egypt: Suez. Hylaeus (Hylaeus) moricei (FrIESE, 1898) - Aliev 1986: 267; Dathe \& Proshchalykin 2018: 46

Prosopis (Prosopis) nigrifacies (BRAMson, 1879) [nomen dubium] - WARNCKE 1972: 755; 1992: 769.

Material examined: Aksaray: Esmekaya, $38^{\circ} 16 \mathrm{~N}$ $33^{\circ} 22 \mathrm{E}, 16.07 .1998$, $0^{\star}$, leg. C. Schmid-Egger. Ankara: Topçu, 39³5N3500E, 29.05.2001, ㅇ, leg W.H. Liebig. Antalya: Konyaaltı, Azmak, $5 \mathrm{~m}, 30.05 .2009$, ơ, ㅇ, leg. H. Özbek (on Mentha longifolia); Konyaalt1 pilaj1, 29.05.2009, 2 o $^{\star}$, 우, leg. J.S. Ascher, H. Özbek, J.G. Rozen (coll. AMNH); Korkuteli, Kızılcadağ, 1500 m, 03.07.1990, 2 우 ㅇ, leg. A.W. Ebmer. Bingöl: Genç $15 \mathrm{~km} \mathrm{~S}, 1400 \mathrm{~m}, 13.08 .1985,2 \sigma^{\top} \sigma^{\star}$, 우, leg. R. Hensen. Bursa: 225 m, 24.08.1985, ơ, leg. R. Hensen; Gemlik, Kurşunlu, 15.07.1997, + , leg. Prudek/Riha; Mudanya, 25 m, 20.08.1985, ơ, ㅇ, R. Hensen. Diyarbakır: 650 m, 09.08.1985, o`, 2 우 우, leg. R. Hensen; 18.07.1977, o`, leg. J. Timmer. Erzincan: Horticultural Research Station, 1300 m, 07.07.2001， $2 \sigma^{\star} \sigma^{\star}$, leg. J.G. Rozen, H. Özbek (coll. AMNH). Erzurum: Atatürk University, 1900 m, 03.07.2007, o* 11.07.2007, 2 o $^{\star}$, +, leg. H. Özbek, J.G. Rozen, J.S. Ascher (coll. AMNH); 13.08.2000, 2 o $^{\star} \sigma^{\star}$ 2 우, leg. S. Pekel (on Melilotus alba); Oltu, Çamlıbel, $22 \mathrm{~km}$ WSW of Oltu, $1700 \mathrm{~m}, 07.07 .2007,2 \sigma^{\star} \sigma^{*}$, leg. J.S. Ascher, H. Özbek, J.G. Rozen (coll. AMNH); Pasinler, Agricultural Research Station, 10.07.2007, ㄲ, 2 우 우, leg. J.S. Ascher, H. Özbek \& J.G. Rozen (coll. AMNH); Tortum, 1700 m, 16.07.1987, ơ , leg. R. Hensen; Kazandere, $1190 \mathrm{~m}, 03.08 .2004,20^{\top} o^{\star}$, 우, leg. S. Çoruh (Eryngium blardieri). Gümüşane: Torul, $1000 \mathrm{~m}, 40^{\circ} 34 \mathrm{~N}$ 39¹7E, 12.07.1985, ơ, leg. M. Schwarz (coll. Schwarz/ Ansfelden); Hakkâri: 1750 m, 09.07.1987, ơ, leg. R. Hensen; Oramar $10 \mathrm{~km} \mathrm{E,} 1700 \mathrm{~m}, 29.06 .1985$, 우 leg. M. Schwarz (coll. Schwarz/Ansfelden); Yüksekova, Varagöz, Sat Dağ 1700 m, 37²5N 44¹3E, 04.07.1983, $\sigma^{\star}$, leg. W. Schacht (coll. Schwarz/Ansfelden). Iğdır: 19.08.1983, ㅇ, leg. J. Timmer. Kars: Sarıkamış, Karakurt, $1600 \mathrm{~m}, 40^{\circ} 10 \mathrm{~N} 42^{\circ} 22 \mathrm{E}, 04.07 .1985,2{\sigma^{\star}}^{\star}$, 우, leg. M. Schwarz (coll. Schwarz/Ansfelden); $40^{\circ} 08 \mathrm{~N}$ $42^{\circ} 21 \mathrm{E}, 1501 \mathrm{~m}, 05.08 .2002,2 \mathrm{o}^{\star} \mathrm{o}^{\star}$, leg. H. Özbek (on Achillea millefolium). Kayseri: İncesu W 5 km, $1100 \mathrm{~m}$, $38^{\circ} 38 \mathrm{~N}$ 035 09E, 27.05.1998, $20^{\star} \sigma^{\star}$, 우, leg. S.M. Blank. Konya: Alaattin Tepesi, 1050 m, 20.06.1987, ơ , ㅇ, leg. R. Hessen; Beyşehir, 1150 m, 18.06.1987, 2 ㅇ 우, leg. R. Hessen. Nevşehir: $2 \mathrm{~km} \mathrm{S,} 1250 \mathrm{~m}, 04.07 .1984$, 우, leg. A.W. Ebmer; Göreme, 38³9N 3452E, 17.08.1984, $0^{\star}$, leg. C. Schmid-Egger; Ürgüp, 1100 m, 11.08.1985, 우, leg. P. van Ooijen. Şanlıurfa: Birecik, 400 m, 06.08.1985, ○, Halfeti, 27.05.1987, ơ , leg. Madl; 400 m, 28.06.1987, đ, leg. R. Hessen; 7 km N Suruç, 400 m, 29.06.1987, $2 \sigma^{\star} \sigma^{\star}$, leg. R. Hessen. Van: 1800 m, 13.07.1987, ơ, ㅇ, leg. R. Hessen; Gevaş, Göllü, 38²43N 4319E, 29.06.1993, ơ, leg. M. Halada (coll. Schwarz/Ansfelden).
Remarks: H. moricei was known from the four provinces Bursa, Iğdır, Kars and Nevşehir. This study includes records from Aksaray, Ankara, Antalya, Bingöl, Diyarbakır, Erzincan, Erzurum, Gümüşhane, Hakkâri, Kayseri, Konya, Şanlıurfa and Van. The collecting sites are at altitudes between sea level (Antalya) and $2000 \mathrm{~m}$ (Erzurum), the flight period extends from the end of May to the end of August with a peak in June and the beginning of July. With the exception of the Aegean region, from which no samples are available, the species can be treated as a common species in Turkey (17 provinces).

Distribution: From Spain in the west to Iran and Central Asia in the east, South to Egypt (DATHE \& ProshCHALYKIN 2018). In Turkey: Bursa, Iğdır, Kars, Nevşehir (WARNCKE 1972, as Prosopis nigrifacies BRAmson, 1879); Erzurum (ÖzBeK 1977, as H. nigrifacies (BrAmson)); Aksaray, Ankara, Antalya, Bingöl, Diyarbakır, Erzincan, Gümüşane, Hakkâri, Kayseri, Konya, Şanlıurfa and Van.

\section{Hylaeus (Hylaeus) nigritus (FABRICIUS, 1798)}

Mellinus nigritus FABRICIUS, 1798: 267, ㅇ. South Europe. Prosopis nigrita (FABRICIUS, 1798) - WARNCKE 1972: 755.

Hylaeus (Hylaeus) nigritus (FABriciUs, 1798) - Aliev 1986: 267; Dathe \& Proshchalykin 2018: 18-20, 50.

Material examined: Erzurum: Palandöken, 22002400 m, 27.07.1986, 4 ㅇ ㅇ , leg. A.W. Ebmer; Umudum Yaylas1, $2100 \mathrm{~m}, \quad 40^{\circ} 04 \mathrm{~N} \quad 41^{\circ} 15 \mathrm{E}, 20.07 .2010$, 우, leg. G. Rozen, J. Ascher, H. Özbek (coll. AMNH). Gümüșhane: Torul, $1000 \mathrm{~m}, 40^{\circ} 34 \mathrm{~N} 39^{\circ} 17 \mathrm{E}, 12.07 .1985$, $0^{\star}$, leg. M. Schwarz (coll. Schwarz/Ansfelden). Hakkâri: Güzeldere Geçidi, 2700-2800 m, 18.07.1986, o, 2 우 으, leg. A.W. Ebmer. Nevşehir: Avanos, 920 m, 07.07.1983, ㅇ, leg. J. Hladil. Van: Başkale $30 \mathrm{~km} \mathrm{~N}, 2700 \mathrm{~m}$, 11.07.1997, ơ, 우, leg. R. Hensen.

Remarks: This study confirms $H$. nigritus from the provinces Erzurum, Gümüşhane, Hakkâri, Nevşehir and Van. Interestingly, all samples were collected in July. The species appears to be sporadically distributed in Turkey. Here it occurs between $900-2800 \mathrm{~m}$ altitude, but is mainly found above $2000 \mathrm{~m}$, and therefore be considered to be a montane species. In Central Europe it is found from the plain to higher altitudes of the low mountains, in the Alps up to $1600 \mathrm{~m}$ (Westrich 2018). Dathe \& ProshChalykin (2018) report it from $4000 \mathrm{~m}$ altitude in Kyrgyzstan and $2700 \mathrm{~m}$ in Tajikistan. This species is only sparsely collected in Turkey (5 provinces).

Distribution: West Palaearctic: Europe from the Iberian Peninsula to Caucasus and Ural, up to $62^{\circ}$ north; Morocco, Asia Minor, Iran, Central Asia (DAThe \& Proshchalykin 2018). In Turkey: Erzurum (Özbek 1977); Gümüşhane, Hakkâri, Nevşehir. Van. 
Hylaeus (Hylaeus) orientalicus (WARNCKE, 1981) Fig. 7

Prosopis (Prosopis) orientalica WARNCKe, 1981: 176-178, 우 o .

Turkey: Kilis. - WARNCKE 1992: 769.

Hylaeus (Hylaeus) orientalicus (WARNCKE, 1981) - ITIS (2020).

Material examined: Antalya: Manavgat $5 \mathrm{~km}$ W, $10 \mathrm{~m}$, 17.06.1987, 2 우 ㅇ, leg. R. Hensen. Mersin: Erdemli $25 \mathrm{~km}$ NW, Toros dağları, $900 \mathrm{~m}, 06.06 .1991$, ơ, leg. S. Kadlec.

Remarks: H. orientalicus is very conspicuous by the oblique impressions in the face of the male, so that the findings of such animals hardly remain hidden. It was described from the provinces Kilis, Adana and Şanlıurfa, as well as from Israel. This study adds specimens from Antalya and Mersin. Accordingly, the species is spread in the eastern Mediterranean and south-eastern Anatolia. Our data suggest that $H$. orientalicus is a thermophilic species mainly from the lowlands, but collected up to $900 \mathrm{~m}$ above sea level. It is sparsely collected in Turkey (5 provinces).

Distribution: This taxon shows a confined distribution range between Israel and Turkey. In Turkey: Adana, Kilis (type locality), Şanlıurfa (WARNCKE 1981); Antalya, Mersin.

\section{Hylaeus (Hylaeus) paulus BRIDWELL, 1919}

Hylaeus paulus Bridwell, 1919: 154, ․ Japan: Honshu.

Hylaeus (Hylaeus) lepidulus Cockerell, 1924: 282-283, o*. Russia: Siberia. - DATHE et al. 1996: 157-163.

Hylaeus (Hylaeus) paulus Bridwell, 1919 - ProshCHALYKIN \&

Dathe 2012: 16. Dathe \& Proshchalykin 2016: 370-371.

Material examined: Antalya: Alanya, 50 m, 28.07.1985, $2 o^{\star} o^{\star}, 4$ 우 ㅇ, leg. R. Hensen; 100 m, 30.07.1985, ơ, leg. R. Hensen; 30.08.1983, , leg. P. v. Ooijen; Alanya, İncekum $20 \mathrm{~km} \mathrm{~W}$ of Alanya, $20 \mathrm{~m}, 07.08 .1985$, $\sigma^{*}$, leg. P. v. Ooijen; Gazipaşa, 30 m, 03.07.1986, $5 o^{\star} o^{*}$, leg. P. v. Ooijen. Balıkesir: Edremit, Ören, $27^{\circ} 02 \mathrm{E} 39^{\circ} 33 \mathrm{~N}$, 04.08.1987, ㅇ, leg. Dollfuss. Bursa: 225 m, 24.08.1985, $2 \sigma^{\star} o^{\star}$, ㅇ, leg. R. Hensen; Armutlu $15 \mathrm{~km} \mathrm{NE}, 50 \mathrm{~m}$, 27.07.1986, 12 ơ đ , leg. P. v. Ooijen; $20 \mathrm{~km}$ E Armutlu, 30 m, 20.08.1985, 우, leg. P. v. Ooijen; Mudanya, 25 m, 25.08.1985, 우, 우, leg. R. Hensen. Denizli: Pamukkale, $20 \mathrm{~km} \mathrm{NE}, 1000 \mathrm{~m}, 01.07 .1986$, †, leg. P. v. Ooijen. Erzurum: Atatürk University Campus, $2000 \mathrm{~m}$, $39^{\circ} 53 \mathrm{~N} 41^{\circ} 14 \mathrm{E}, 30.06 .2007,0^{\top}$, 우, leg. J.S. Ascher, H. Özbek J.G. Rozen (coll. AMNH). Hakkâri: 1750 m, 09.07.1987, ㅇ , leg. R. Hensen; N 2000 m, 21.07.1986, 운, leg. A.W. Ebmer. Hatay: İskenderun, Belen, 28.08.1983, $3 o^{\top} o^{\star}$, 우, leg. J. Timmer. İzmir: Efes, 18.05.1992, 2 우 우, leg. W.H. Liebig. Mersin: Baland $1 z, 36^{\circ} 20 \mathrm{~N} 33^{\circ} 46 \mathrm{E}$, 1000 m, 06.06.1985, , leg. Aspöck/Rausch; 35 km
NNW Kargican, 200 m, 04.08.1985, + , leg. R. Hensen. Muğla: Fethiye, Ölüdeniz, 25.07.1986, 2 o $^{\star}$, , 30.07.1985, 3 우 ㅇ leg. P. v. Ooijen; Marmaris $20 \mathrm{~km} \mathrm{~W}$, 17.08.1983, 2 o $^{\star}$, leg. A. Link. Trabzon: Çavuşlu, Eynesil, 13.08.1983, ㅇ, leg. P. v. Ooijen. Yalova: 12 km SW, Termal, 30 m, 25.08.1985, ơ, ㅇ , leg. R. Hensen.

Remarks: $H$. paulus is recorded from all geographical regions of the country except Central Anatolia. It apparently has a sporadic distribution. The collection sites show that the samples were mostly collected in open areas at altitudes between 20 and $2000 \mathrm{~m}$ and are mainly concentrated in coastal areas. The flight period is quite long, from May to the end of August; possibly more than one generation a year. It is collected moderately in Turkey (12 provinces, Fig. 16.5).

Distribution: Euro-Siberian species found from Central Europe via the Asian part of Russia to Mongolia, China and Japan (Dathe \& Proshchalykin 2018). New for the Turkish fauna, Antalya, Balıkesir, Bursa, Denizli, Erzurum, Hakkâri, Hatay, İzmir, Mersin, Muğla, Trabzon, Yalova

\section{Hylaeus (Hylaeus) scutellaris MoRaWITz, 1874}

Hylaeus scutellaris Morawitz, 1874: 176, ․ . Azerbaijan: Baku. Prosopis (Prosopis) scutellaris (MoRAwITZ, 1874) - WARNCKe 1972: 754.

Hylaeus (Hylaeus) scutellaris MoRawitz, 1874 - Aliev 1986: 267; Dathe \& Proshchaly Kin 2018: 64.

Material examined: Antalya: Konyaaltı Beach, $2 \mathrm{~m}$, $36^{\circ} 5154 \mathrm{~N} 30^{\circ} 3839 \mathrm{E}, 29.05 .2009$, o ${ }^{\star}$, leg. J.S. Ascher, H. Özbek, J.G. Rozen; Döşemealtı, Çı̆̆lık, 317 m, 02.06.2009, ð, leg. J.S. Ascher, H. Özbek.

Remarks: H. scutellaris was only known from the Eastern Anatolian region (Iğdır and Kars), but according to the present study, interestingly Antalya has to be added to the distribution list. The species shows a sporadic distribution. It is collected only sparsely (3 provinces) in Turkey.

Distribution: South-eastern Europe, Russia, Turkey, Armenia, Azerbaijan, Afghanistan, Iran, Pakistan, Central Asia (Dathe \& Proshchalykin 2018). In Turkey: Iğdır, Kars (WARncKe 1972), Antalya.

\section{Hylaeus (Hylaeus) sidensis (WARNCKE, 1981)} Fig. 8

Prosopis (Prosopis) sidensis WARNCKe, 1981: 175-176, 웅. Turkey: Side. - WARNCKE 1992: 769.

Hylaeus (Hylaeus) sidensis (WARNCKE, 1981) - ITIS (2020). 


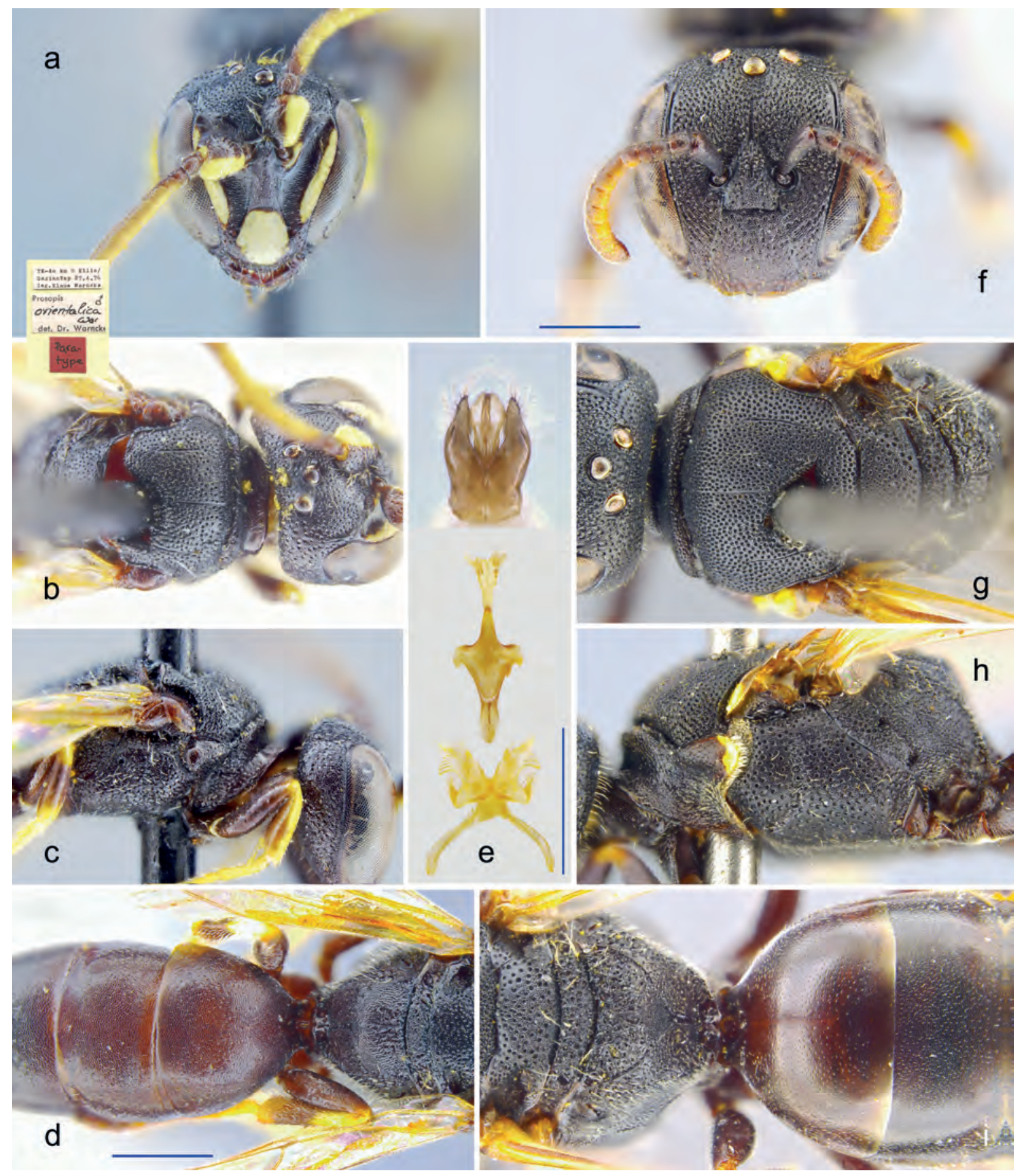

Fig. 7: H. (Hylaeus) orientalicus (WARncke, 1981). Paratype male (Kilis): a-face, b-mesonotum, c-mesopleuron, d-propodeum and metasoma, e-terminalia. Female (Antalya): f-face, $\mathbf{g}$-mesonotum, $\mathbf{h}$-mesopleuron, $\mathbf{i}$-propodeum and metasoma. - Scale bar $0.5 \mathrm{~mm}$.

Material examined: Adana: Pozant1, $37^{\circ} 25 \mathrm{~N} 34^{\circ} 52 \mathrm{E}$ 06.07.1983, 2 우 ㅇ, leg. J. Hladil (coll. Kocourek). Hatay: Payas, 10.04.2002, $3 \sigma^{\star} \sigma^{*}, 2$ 우 잉 leg P. Bogusch (coll. P. Bogusch); Samandağı $30 \mathrm{~km} \mathrm{N,} \mathrm{Nur} \mathrm{dağları,}$ 06.07.1996, ㅇ, leg. Brechtel/Ehrmann.

Remarks: H. sidensis was described from Antalya, Adana, Kilis, İzmir and Mersin as well as from Israel and Lebanon (WARNCKE 1981). The present study adds the province Hatay. The localities from which the evidence comes show that it is a thermophilic species of plains, mainly at altitudes below $1000 \mathrm{~m}$, frequently found in the Mediterranean coastal region. It is an early-flying bee, from the beginning of April to July. This small and dainty species with the striking formation of the male gonoforcipes is only sparsely collected in Turkey (6 provinces). 


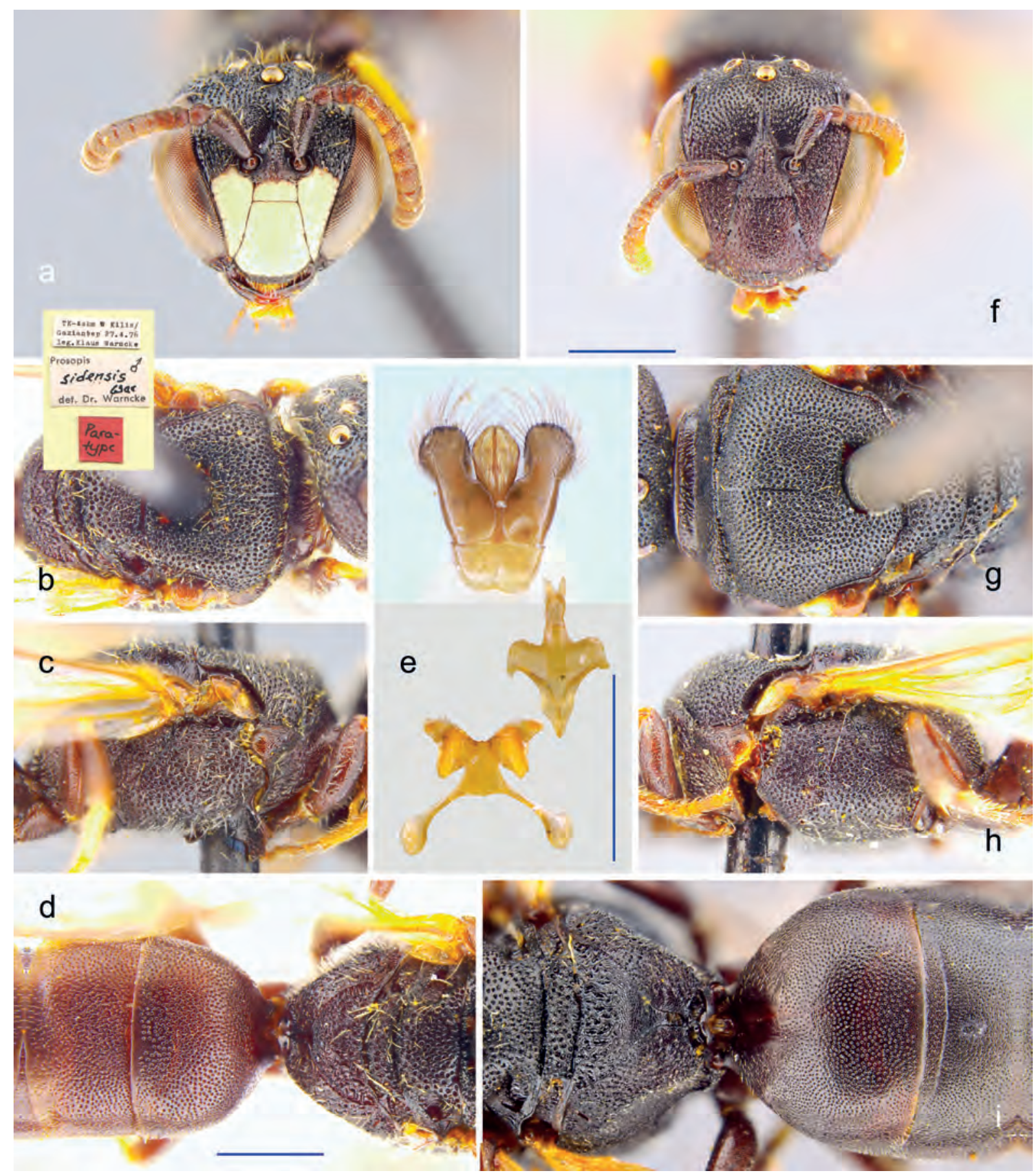

Fig. 8: H. (Hylaeus) sidensis (WARncKe, 1981). Paratype male (Kilis): a-face, b-mesonotum, c-mesopleuron, d-propodeum and metasoma, e-terminalia. Female (Amanus): $\mathbf{f}$-face, $\mathbf{g}$-mesonotum, $\mathbf{h}$-mesopleuron, $\mathbf{i}$-propodeum and metasoma. - Scale bar $0.5 \mathrm{~mm}$.

Distribution: Eastern Mediterranean (Greece, Turkey, Lebanon, Israel, Syria). In Turkey: Adana, Antalya (type locality), Kilis, İzmir, Mersin (WARnCKe 1981), Hatay.

Hylaeus (Hylaeus) tardus (WARnCKE, 1981)

Prosopis (Prosopis) tarda WARnCKe, 1981: 173-174, 우 ơ. Iran: Isfahan.
Hylaeus (Hylaeus) tardus (WARNCKE, 1981) - ITIS (2020).

Material examined: Adıyaman: Nemrut Mt. National Park, Karadut, 2000 m, 3756N 38²7E, 02.07.1993, $50^{\top} o^{\top}, 18$ 우 우, leg. Halada/Jirousek (coll. Schwarz/

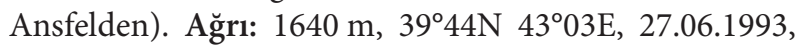
$0^{\star}$, leg. M. Halada(coll. Schwarz/Ansfelden). Balıkesir: Edremit, Öran $27^{\circ} 02 \mathrm{E} 39^{\circ} 33 \mathrm{~N}, 04-15.08 .1987$, ơ ${ }^{\star}$, 우, leg. Dollfuss. Diyarbakır: $650 \mathrm{~m}, 09.08 .1985,{ }^{\star}$, leg. 
R. Hensen. Mardin: $1000 \mathrm{~m}, 02.07 .1987,3 \sigma^{\star} o^{*}$, leg. R. Hensen. Sivas: Gürün, Suğul, 1400 m, 31.07.1986, o, 2 ㅇ ㅇ, leg. A.W. Ebmer. Şanlıurfa: Birecik, $400 \mathrm{~m}$, 06.08.1985, 11 ơ o, 9 우 우, leg. R. Hensen; Halfeti, 425 m, 07.08.1985, $17 \sigma^{\star} o^{\star}$, o , leg. R. Hensen; $400 \mathrm{~m}, 28.06 .1987$, $4 o^{*} o^{*}$, ơ , leg. R. Hensen; Suruç, 400 m, 29.06.1987, o*, ㅇ, leg. R. Hansen. Van: Başkale, 2200 m, 10.07.1987, ơ, leg. R. Hensen.

Remarks: $H$. tardus was described from Iran (WARNCKE 1981). According to "Discover Life" (Ascher \& Pickering 2019), the species also occurs in Turkey and Azerbaijan, but we could not find out more about the exact localities. The available records show that this species is widespread in south-eastern Anatolia, near to the border with Iran. Thus it could be considered as an inhabitant of warm and dry places, but it also occurs in cool and mountainous locations (Van, $2200 \mathrm{~m}$ ). Balıkesir is the most western recorded locality of this species. It is moderately collected in Turkey (8 provinces).

Distribution: Iran (type locality), Azerbaijan, Turkey: Adıyaman, Ağrı, Balıkesir, Diyarbakır, Mardin, Sivas, Şanlıurfa, Van.

\section{Hylaeus (Hylaeus) torquatus (WARNCKE, 1992)} Fig. 9

Prosopis (Prosopis) torquata WARNCKe, 1992: 784-785, i o Turkey: Midyat (Mardin).

Hylaeus (Hylaeus) torquatus (WARNCKe, 1992) - ITIS (2020).

Remarks: In the original description, WARNCKE (1992: 769) classifies the species in the subgenus "Prosopis" (= Hylaeus s. str.) between H. tyrolensis and H. funereus. According to our understanding, these species belong to different subgenera. In fact, $H$. torquatus has special characteristics sui generis, which call both classifications into question. We recognize a closer comparability only with $H$. sidensis, with which it shares the following characters: the short, apically broadened gonoforcipes of the males, in both sexes the dense strong punctation of the head and mesosoma, so that the sculpture appears matt; the lateral areae of the propodeum are arched without posterior ridge; the white mask is dorsally transversally truncated. The face of the female is completely black.

WARNCKE (1992) described H. torquatus from Mardin and recorded it simultaneously from Hakkâri and Siirt as well as from Syria. Since then the species has not been found again. It is only sparsely collected in Turkey (3 provinces).

Distribution: Syria; Turkey: Hakkâri, Siirt, Mardin.

\section{Hylaeus (Hylaeus) trifidus (AlfKen, 1936)}

Prosopis trifida Alfken, 1936: 52-53, o o o. Lebanon: Said Neil. Prosopis (Prosopis) trifida AlfKen, 1936 - WARnCKE 1981: 191-192.

Hylaeus (Hylaeus) trifidus (Alfken, 1936) - Dathe 2015: 225-227.

Material examined: Adıyaman: Nemrut Mt National Park, Karadut, 2000 m, 3756N 38 ${ }^{\circ} 47 \mathrm{E}$, 02.07.1993, ơ leg. M. Halada (coll. Schwarz/Ansfelden). Balıkesir: Ayvalık, Arkent 28.06.1993, §, leg. P. Tyrner. Burdur: Çeltikçi, 02.09.1983, 6 o $^{*}$, leg. J. Timmer. Eskişehir: Mihalgazi, Sakarulıca, 06.07.1997, ơ , ㅇ, leg. Prudek/ Riha. Hakkâri: Beytüşşebap, Habur Deresi, 37³2N $43^{\circ} 12 \mathrm{E}, 1100 \mathrm{~m}, 01.08 .1983$, o* leg. W. Schacht (coll. Schwarz/Ansfelden); Konya: Güneysınır, Gürağaç, 1020 m, 06.08.2002, ㅇ, leg. M. Kesdek. Mardin: 1000 m, 02.07.1987, $5 \sigma^{\star} \sigma^{*}, 3$ ㅜ, R. Hensen. Mersin: Silifke, Uzuncaburç, 400 m, 02.08.1985, $2 \sigma^{\top} \sigma^{\star}$, leg. R. Hensen; Kargican, 200 m, 04.08.1985, 6 우 이 leg. R. Hensen. Muğla: Bodrum, Salkamış, 13-25.07.2001, $37^{\circ} 02 \mathrm{~N}$ $27^{\circ} 25 \mathrm{E}, 46$ o $^{\star} 0^{\star}, 20$ 우 우, leg. F. Burger (on Polygonum sp.); Milas, Yatağan, $37^{\circ} 17 \mathrm{~N} 27^{\circ} 56 \mathrm{E}, 500 \mathrm{~m}, 05.06 .1984$, ơ leg. Vogtenhuber (coll. Schwarz/Ansfelden). Nevşehir: Avanos, 07.07.1983, ơ, leg. J. Hladil; Göreme, 38³9N $34^{\circ} 52 \mathrm{E}, 17.07 .1998$, ơ leg. C. Schmid-Egger; Hacıbektaş, Mucur, 1100 m, 20.07.1984, ơ, leg. A.W. Ebmer; Zelve, 1050, 04-18.07.1984, 4 우 우, leg. A.W. Ebmer; Zelve, 1000 m, 22.06.1987, ㅇ, leg. R. Hensen.

Remarks: H. trifidus can be regarded as an inhabitant of the lowlands, but it also occurs in mountainous locations (Adiyaman, $2000 \mathrm{~m}$ ). It prefers warm and dry habitats, mainly in steppes and open areas. It is moderately collected in Turkey (12 provinces).

Distribution: Greece (Lesvos, Chio), Israel, Egypt (Sinai) (DATHE 2015); Iran has to be deleted (WARNCKE 1981: 192). Turkey: Ankara, Konya, Mersin (WARnCKE 1972, 1981), Adıyaman, Balıkesir, Burdur, Eskişehir, Hakkâri, Konya, Mardin, Muğla, Nevşehir.

\section{Hylaeus (Hylaeus) trisignatus MorawITz, 1876}

Hylaeus trisignatus Morawitz, 1876: 279-280, 우 o . Tajikistan: Iskander.

Prosopis trisignatus (MoRAWITZ, 1876) - WARNCKe 1981: 151-152.

Hylaeus (Hylaeus) trisignatus MoRawitz, 1876 - DATHE \& Proshchalykin 2018: 65.

Material examined: Hakkâri: Varagöz, Sat Dağı, 1700 m, $37^{\circ} 25 \mathrm{~N} 44^{\circ} 13 \mathrm{E}, 04-08.08 .1983$, 우, leg. W. Schacht.

Remarks: The occurrence of $H$. trisignatus has so far been considered limited to Central Asia. It is remarkable 


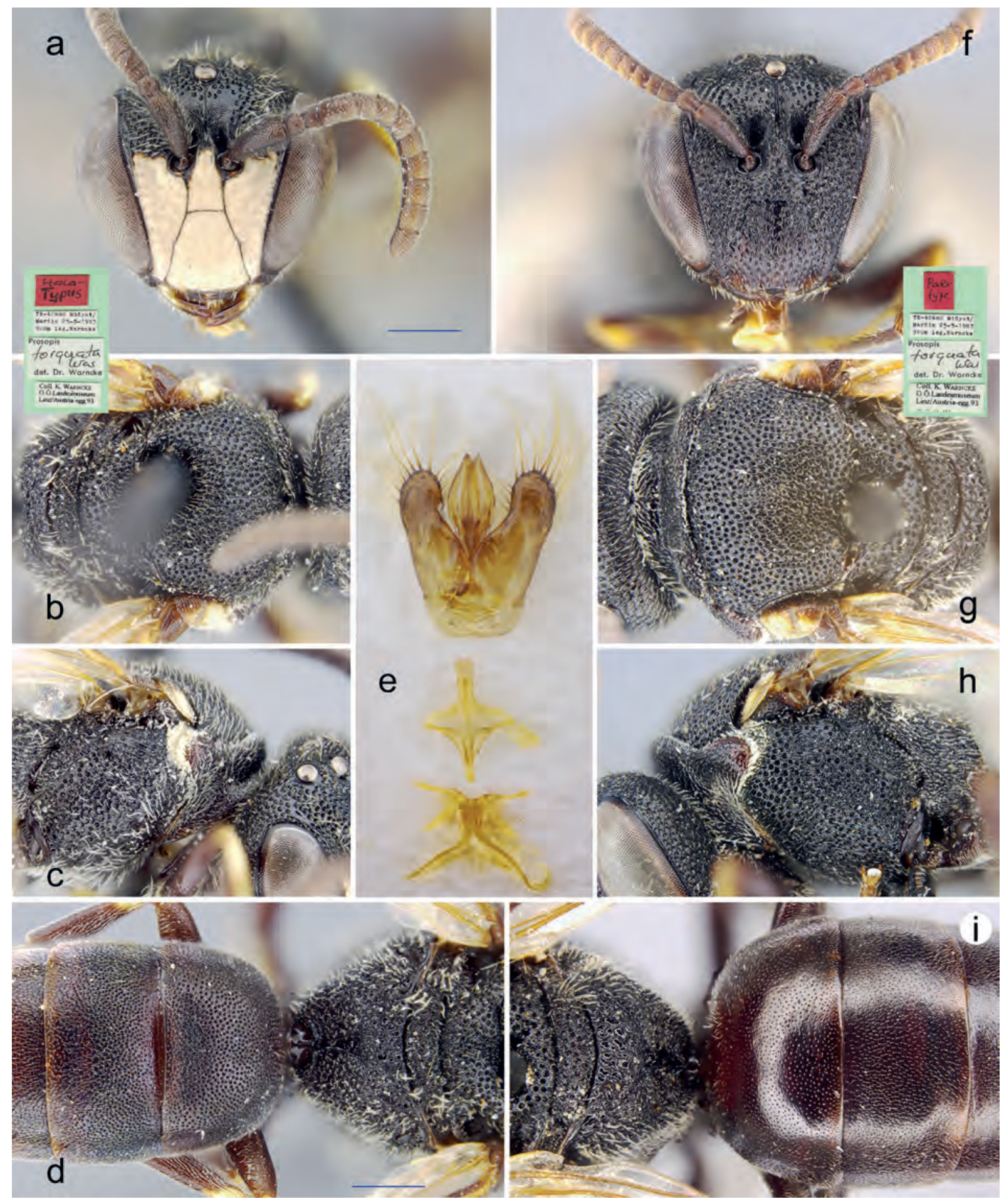

Fig. 9: H. (Hylaeus) torquatus (WARncKe, 1992). Holotype male: a-face, b-mesonotum, c-mesopleuron, d-propodeum and metasoma, e-terminalia. Paratype female: $\mathbf{f}$-face, $\mathbf{g}$-mesonotum, $\mathbf{h}$ - mesopleuron, $\mathbf{i}-$ propodeum and metasoma. - Scale bar $0.5 \mathrm{~mm}$.

that this species is now documented as new for Turkey by a sample from Hakkâri. Hakkâri is the westernmost distribution point of this species. It is rarely recorded from Turkey (1 province, Figs 16.6, 17.5).

Distribution: Tajikistan, Kyrgyzstan (Proshchalykin \& DATHe 2018); Turkey: Hakkâri.
Hylaeus (Hylaeus) trochilus (WARNCKE, 1992) Fig. 10

Prosopis (Prosopis) kurda trochila WARNCKE, 1992: 792-793, $0^{\top}$ ㅇ. Turkey: Nigde.

Hylaeus (Hylaeus) trochilus (WARNCKE, 1992) - ITIS (2020). 


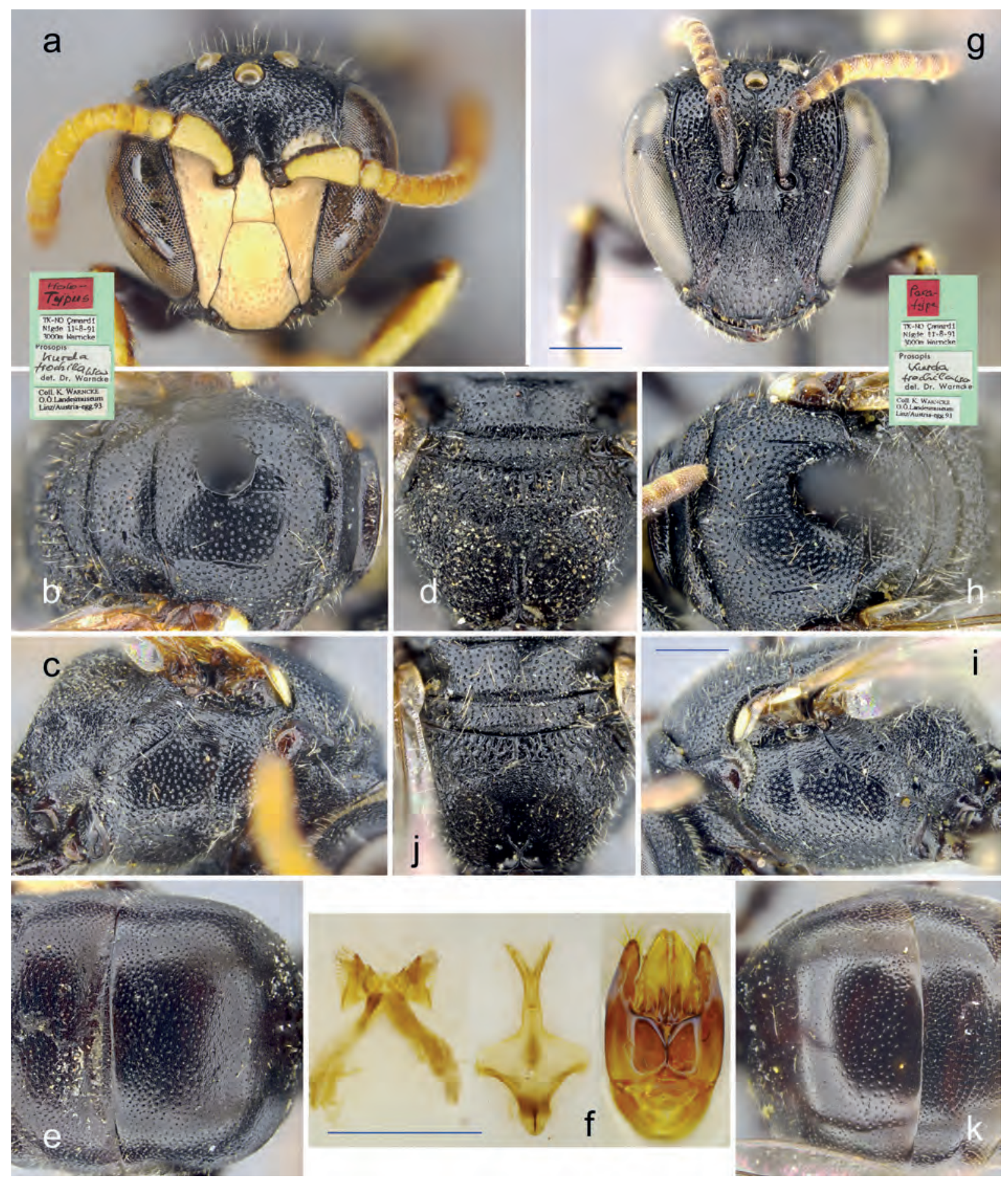

Fig. 10: H. (Hylaeus) trochilus (WARncKe, 1992). Holotype male: a-face, b-mesonotum, c-mesopleuron, d-propodeum, e-metasoma, f-terminalia. Paratype female: $\mathbf{g}$-face, $\mathbf{h}$-mesonotum, $\mathbf{i}$-mesopleuron, $\mathbf{j}$-propodeum, $\mathbf{k}$-metasoma. - Scale bar $0.5 \mathrm{~mm}$.

Remarks: In the males of $H$.trochilus, the face is impressed transversely in a bow below the antenna bases, similar to H. kurdus. In contrast to H. kurdus, however, the scape of males is richly marked with white, its outline basally less curved and expanded, with width less than half the length of the scape, apically not flattened. The side spots run along the orbits, they are rounded at the top, not emarginate; there are also no smooth surfaces on the frons. Inappropriate preparation of the terminalia by Warncke caused the loss of sternum 7 , and sternum 8 is a fragment.

Apart from the specimens of the type series from Niğde at $3000 \mathrm{~m}$ altitude $\left(2 \sigma^{\top} \sigma^{x}\right.$, o $)$, no other samples were collected. We assume that it is a species especially of the 
high mountain regions, that has to be searched for at suitable places. It is rarely recorded from Turkey (1 province, Fig. 17.6).

Distribution: Turkey: Niğde.

\section{Hylaeus (Hylaeus) tyrolensis FöRSTER, 1871}

Hylaeus tyrolensis Förster, 1871: 980-981, ․ Austria: Tyrol. Prosopis (Prosopis) tyrolensis (FöRster, 1871) - WARNCKE 1972: 756; 1992: 768.

Hylaeus (Hylaeus) tyrolensis Förster, 1871 - DAthe et al. 2016: 26,41

Material examined: Adıyaman: 20 km E Gölbaşı, 600 m, $37^{\circ} 44 \mathrm{~N} 37^{\circ} 55 \mathrm{E}, 12.05 .2002$, o, 4 우 우, leg. J.G. Rozen, H. Özbek (coll. AMNH). Antalya: Çiğlik, 317 m, 02.06.2009, †, leg. J.S. Ascher, H. Özbek; Termassos, $500 \mathrm{~m}, 37^{\circ} 02 \mathrm{~N} \mathrm{30} 28 \mathrm{E}, 24.05 .2009,6 \mathrm{o}^{\star} \mathrm{o}^{\star}$, leg. J.S. Ascher, H. Özbek (coll. AMNH). Bursa: Çağlayan, 10.07.1997, 2 우 우, leg. Prudek/Riha; Kurşunlu, 15.07.1997, ơ 우, leg. Prudek/Riha. Erzurum: $5 \mathrm{~km}$ NE of Pasinler, $1700 \mathrm{~m}$, 10.07.2007, ơ, leg. J.S. Ascher, J.G. Rozen, H. Özbek (coll. AMNH). Osmaniye: Hassa, $6 \mathrm{~km} \mathrm{~W}$. Hassa, $850 \mathrm{~m}$, $36^{\circ} 49 \mathrm{~N} 36^{\circ} 29 \mathrm{E}, 13.05 .2002,3$ o $^{\top}$ o $^{\circ}$ 우 우, leg. J.G. Rozen, H. Özbek (coll. AMNH).

Remarks: The available records show that H.tyrolensis has a rather sporadic distribution, centred on the Mediterranean. Erzurum is the most eastern point of distribution of this species. It occurs from sea level (Bursa) up to about $1700 \mathrm{~m}$ in both warmer and cooler regions, but preferring warmer ones. The flight period lasts from May to August. It is collected moderately in Turkey (8 provinces).

Distribution: West Palaearctic, Alps to Asia minor. In Turkey: Çanakkale, Mersin, Hakkâri (WARNCKE 1972, 1992), Adiyaman, Antalya, Bursa, Erzurum and Osmaniye.

\section{Subgenus Koptogaster ALFKEN, 1912}

\section{Hylaeus (Koptogaster) bifasciatus (JURINE, 1807)}

Prosopis bifasciata JuRIne, 1807: 220, pl. 11, ․ . South Europe.

Prosopis (Koptogaster) bifasciata JURINE, 1807 - WARNCKE 1972: 752; 1981: 190; 1992: 769.

Hylaeus (Koptogaster) bifasciatus (Jurine, 1807) - Aliev 1986: 268 .

Material examined: Konya: Çumra, 1016 m, 17.07.2000, o, Güneysınır, Gürağaç, 06.07.2000, ㅇ, leg. M. Kesdek. Sinop: Ayanc1k, 23.07.1977, क, leg. H. Özbek (Onopordum sp.).
Remarks: The provinces Konya and Sinop are to be added to the distribution area of $H$. bifasciatus. It has a sporadic distribution, and is sparsely collected in Turkey (6 provinces).

Distribution: Southern Europe, Ukraine, Georgia, Israel, Malaysia (Ascher \& Pickering 2019). In Turkey: Ankara, Manisa, Mersin, Şanlıurfa (WARnCKE 1972), Konya, Sinop.

\section{Hylaeus (Koptogaster) punctulatissimus SMIтH, 1842}

Hylaeus punctulatissimus SмIтн, 1842: 58, क o . England: Coombe.

Prosopis (Koptogaster) punctulatissima (SMITH, 1842) WARNCKE 1972: 752; 1981: 191; 1992: 769.

Hylaeus (Koptogaster) punctulatissimus Sмiтн, 1842 - Aliev 1986: 269.

Material examined: A ğrı: $1700 \mathrm{~m}, 39^{\circ} 44 \mathrm{~N} 43^{\circ} 03 \mathrm{E}$, 27.06.1993, ox, leg. Jirousek (coll. Schwarz/Ansfelden). Antalya: WNW 25 km, Güllük Dağı (Bey Dağları), 36.59N 030.27E, $950 \mathrm{~m}, \quad 04.06 .1998,2 o^{\star} o^{\star}$, leg. S.M. Blank; Beydağları, 28 km S Elmalı, 1650-1750 m, 04.07.1990, $20^{\top} \sigma^{\star}$, 우, leg. A.W. Ebmer; $7 \mathrm{~km} \mathrm{~S}$ of Kargin, 24.05.2009, o, leg. J. Ascher; 02.06.2009, 5 o $^{\star}$ o $^{\star}$, 4 ㅇ 우, leg. J.S. Ascher, H. Özbek (coll. AMNH). Kars: Sarıkamış, Karakurt, TCK Çeşmesi 1500 m, 4008N $42^{\circ} 21 \mathrm{E}, 08.06 .2005$, ơ, leg. H. Özbek. Kayseri: Develi, Bakırdağ, Gezbeli Geçidi, 1750-1900 m, 06.07.1984, 우, leg. A.W. Ebmer. Nevşehir: Ürgüp, 1200 m, 24.06.1987, ơ, leg. R. Hensen. Niğde: Karakışlakçı 2 km S, 1200 m, 07.07.1995, + , leg. Y. Barbier (on Allium sp.). Van: Gevaş, Göllü, 1800 m, 3843N 4319E, 29.06.1993, o, leg. M. Halada (coll. Schwarz/Ansfelden).

Remarks: H. punctulatissimus was previously known only from Konya. With the present study six further provinces are added to its known distribution. It is recorded for the first time from the regions East Anatolia and Mediterranean. In Turkey the distribution of this taxon is mainly limited to eastern and central Anatolia. It is a species of open land and mountains that is recorded above $900 \mathrm{~m}$ and occurs more frequently at about $1200 \mathrm{~m}$ altitude. Interestingly, it has not been found in Erzurum since the 1970s, despite intensive collection work. Another remarkable thing: The abundance is very low, except for Antalya, each province is only represented by one sample. The flight period lasts from the end of May to July, with a peak in June. It is moderately collected in Turkey (8 provinces).

Distribution: Western Palaearctic. In Turkey: Konya (WARnCKe 1972), Ağrı, Antalya, Kars, Kayseri, Nevşehir, Niğde, Van. 


\section{Hylaeus (Koptogaster) tetris DATHE, 2000}

Hylaeus (Koptogaster) tetris DATHE, 2000: 171-172, ơ ㅇ. Turkey: Isparta.

Remarks: H. tetris was described from Isparta and Kayseri, represented by two specimens only ( $\sigma^{*}$, + ) (DATHE 2000). No additional material has been collected since the time of the description. Currently it appears to be endemic in Turkey, but there are no data to assess the threat situation. It is rarely registered in Turkey (2 provinces, Fig. 17.V).

Distribution: Turkey: Isparta, Kayseri.

\section{Subgenus Lambdopsis PoPOv, 1939}

\section{Hylaeus (Lambdopsis) crassanus (WARNCKE, 1972)}

Hylaeus politus FöRster, 1871: 1050-1051, ㅇ. Switzerland: Chur.

Hylaeus (Lambdopsis) crassanus FöRster, 1871. - DATHE 1980: 272.

Prosopis (Lambdopsis) crassana WARNCKe, 1972: 767 (nom. nov). - WARNCKe 1992: 763.

Remarks: H. crassanus is a species previously known mainly from European mountain regions. WARNCKE (1972) only took it up about 50 years ago from İstanbul (Şile, Fig. 17.7). Despite intensive collecting in the whole country, no additional sample was found; possibly it is extinct in Turkey.

Distribution: Europe from Iberia to the Balkans. Turkey: İstanbul (WARNCKE 1972).

\section{Hylaeus (Lambdopsis) dilatatus (KıRBY, 1802)}

Melitta dilatata KIRBy, 1802: 38-39, ơ. England: Suffolk, Barham.

Prosopis (Lambdopsis) annularis (KIRBY, 1802) - WARNCKe 1972: 767.

Prosopis (Lambdopsis) annularis elbursa WARNCKE, 1981: 170-171, (from Hakkâri). - WARNCKE 1992: 763.

Hylaeus annularis auctorum nec Kirby, 1802 - Aliev 1986: 268. Hylaeus (Lambdopsis) dilatatus (Кirвy, 1802) - Notton \& DATHE 2008: 1863-1865.

Material examined. Ağrı: 20 km N 2000 m, 24.07.1986, ㅇ , leg. A.W. Ebmer; Cumaçay, 2300 m, 23.07.1996, 2 우 우, leg. P. Rasmont (on Cirsium arvense). Artvin: Yusufeli, Kaçkardağları, Yaylalar, 1800 m, 17-19.07.1995, ${ }^{\star}$, leg. Gelbrecht/Schwabe (on Anchusa sp.). Bursa: Kurşunlu, 15.07.1997, 우, leg. Prudek/Richa. Erzurum: Palandöken, 2200-2400 m, 27.07.1986, ơ, 2 우 우, leg. A.W. Ebmer; Oltu, Başaklı, Karadağ Mt., 2100 m,
24.07.2005, $2 \sigma^{\star} \sigma^{\star}$, leg. H. Özbek (on Achillea millefolium); Çamlıbel, $22 \mathrm{~km}$ WSW of Oltu, 08.07.2007, 2 o o $^{\star}$, leg. J.S. Ascher, H. Özbek, J.G. Rozen (coll. AMNH); Pazaryolu, 27.06.2008, 2 ㅇ 우, leg. J.G. Rozen, H. Özbek (coll. AMNH). Erzincan: Muti köprüsü, 1200 m, 26.09.1979, † leg. H. Özbek (on Heliotropium europaeum). Hakkâri: Yüksekova $15 \mathrm{~km} \mathrm{E,} \mathrm{2100-2200} \mathrm{m,}$ 20.07.1986, $20^{\star} 0^{\star}, 2$ 우, leg. A.W. Ebmer; Varegöz, $37^{\circ} 25 \mathrm{~N} 44^{\circ} 13 \mathrm{E}, 1750 \mathrm{~m}, 06.08 .1986$, ㅇ, leg. SM. Blank (coll. Schwarz/Ansfelden); Kaval, Süvarihalil Geçidi, 2200-2400, 22.07.1986, ơ, leg. A.W. Ebmer. Muğla: Köyceğiz, 13.06.1987, ơ, P.H. Doesburg. Sinop: 100 m, 25.07.1977, ơ, leg. H. Özbek (on Onopordum sp.).

Remarks: NotTon \& DAthe (2008) recognized during a revision of the Kirby collection that the previously widely-used name Hylaeus annularis had to be replaced by Hylaeus dilatatus. The type locality of Prosopis annularis elbursa WARNCKE, 1981 (a synonym of $H$. dilatatus (KIRBY) according to our understanding), is not the Elburs mountains in Iran, but the province Hakkâri $(2600-3000 \mathrm{~m})$ in Turkey. Here seven provinces are newly added to the recorded distribution. The species shows a sporadic distribution, recorded from sea level (Muğla) up to a main occurrence at high altitudes (2000-3000 m). Achillea millefolium, Cirsium arvense, Heliotropium europaeum and Onopordum sp. are noted as visited plants. $H$. dilatatus is collected moderately in Turkey (10 provinces).

Distribution: Palaearctic (Russia, Europe, North Africa, Azerbaijan, Turkey, Kazakhstan (DATHE \& ProshChalykin 2018). In Turkey: Bolu, Hatay (WARnCKe 1972) as Prosopis annularis (KIRBY, 1802); Hakkâri as Prosopis annularis elbursa WARNCKE (WARNCKE 1981); Ağrı, Artvin, Bursa, Erzincan, Erzurum, Muğla and Sinop.

\section{Hylaeus (Lambdopsis) euryscapus FöRSTER, 1871}

Hylaeus euryscapus Förster, 1871: 909-910, ơ. Hungary.

Prosopis (Lambdopsis) euryscapa (FöRster, 1871) - WARNCKE 1972: 767; 1992: 768.

Hylaeus (Lambdopsis) euryscapus Förster, 1871 - Aliev 1986: 268.

Material examined: Ardahan: Posof, Aşıkzülali, 1100 m, 25.07.2005, 2 ㅇ , leg. C. Güçlü (on Eryngium creticum). Aksaray: Esmekaya, $38^{\circ} 16 \mathrm{~N} 33^{\circ} 22 \mathrm{E}$, 16.07.1998, 우, leg. C. Schmid-Egger; Ihlara, $27 \mathrm{~km} \mathrm{NE}$, $38^{\circ} 14 \mathrm{~N} 34^{\circ} 18 \mathrm{E}, 18.07 .1998,20^{\star} 0^{\star}$, leg. C. Schmid-Egger. Artvin: Yusufeli, Demirkent, $1600 \mathrm{~m}, 01.09 .1995$, 우, leg. M. Kraus. Çorum: Mecitözü, 750 m, 05.06.2002, ㅇ, leg. W.H. Liebig. Erzurum: Atatürk University Campus, 2000 m, 09.08.1970, ㅇ, leg. H. Özbek (on Melilotus officinalis); 03.09.1987, ơ, leg. R. Hayat; 11.07.2007, ơ, 우, leg. J.S. Ascher, H. Özbek, J.G. Rozen (coll. AMNH); 
16.07.2003, 2 우 이 leg. J.G. Rozen and H. Özbek; $41 \mathrm{~km} \mathrm{~W}$ of Erzurum, Aşkale civarı, 02.07.2007, 2 ๙ o $^{\star}$, leg. J.S. Ascher, H. Özbek, J.G. Rozen (coll. AMNH); Pasinler, 26.07.1997, ㅇ, leg. H. Özbek. Hakkâri: Yüksekova, 15 km E, 2100-2200 m, 20.07.1986, ơ, ㅇ, leg. A.W. Ebmer. Kayseri: Develi, Bakırdağı, 1700 m, 06.07.1984, ㅇ, leg. A.W. Ebmer; $38^{\circ} 22 \mathrm{~N} 35^{\circ} 29 \mathrm{E}, 1400 \mathrm{~m}$, 02.05.1985, ơ , leg. Aspöck/Rausch. Konya: Güneysınır, Gürağaç, 1020 m, 06.07.2000, $2 \sigma^{\star} \sigma^{*}$, leg. M. Kesdek; Bozkır, Sarıoglan, $1000 \mathrm{~m}, 25.07 .2003,20^{\star} o^{\star}$, leg. M. Kesdek; Eflatun pinarı, 37 $51 \mathrm{~N} 31^{\circ} 37 \mathrm{E}, 15.07 .1998$, $30^{\star} \sigma^{\star}$, leg. C. Schmid-Egger. Muğla: Köyceyiz, 14.06.1987, ơ, leg. P.H. van Doesburg. Nevşehir: Avanos, Zelve, 1060 m, 17.07.1984, ㅇ, leg. A.W. Ebmer. Niğde: 2 km S, 1250, 04.08.1984, , leg. A.W. Ebmer; Maden, Çiftehan, 1700-1800 m, 17.07.1990, ơ, leg. A.W. Ebmer; Göreme, 38³9N 3453E, 17.07.1998, ơ, leg. C. Schmid-Egger. Şanlıurfa: Halfeti, 08.06.1983, o, leg. J. Schmidt.

Remarks: Ten provinces can be added here to the range of H. euryscapus. The species occurs in all geographical regions, from sea level to $2200 \mathrm{~m}$ altitude. The flight period extends from the beginning of May to August with a peak in July. It is moderately recorded in Turkey (16 provinces).

Distribution: Western Palaearctic. In Turkey: Balıkesir, Denizli, Konya, İstanbul, Tekirdağ (WARNCKE 1972); Erzurum (Özbek 1977); Ardahan, Aksaray, Artvin, Çorum, Hakkâri, Kayseri, Muğla, Nevşehir, Niğde, Şanlıurfa.

\section{Hylaeus (Lambdopsis) scutellatus (SPINOLA, 1838)}

Prosopis scutellata Spinola, 1838: 506-507, ․ Egypt.

Prosopis (Lambdopsis) scutellata SPINOLA, 1838 - WARNCKE 1972: 768; 1992: 764.

Hylaeus (Lambdopsis) scutellatus (SPINOLA, 1838) - Aliev 1986: 268.

Material examined: Adıyaman: $20 \mathrm{~km} \mathrm{SE}, 680 \mathrm{~m}$, 10.05.2002, 2 o $^{\star}$, leg. H. Özbek; Gölbașı, Celigölü, 900 m, 21.06.1985, ㅇ, leg. W. Schacht. Antalya: Alanya, 50 m, 28.07.1985, ơ , leg. R. Hensen; Konakl1 10 km W, $36^{\circ} 58 \mathrm{~N} \mathrm{31}{ }^{\circ} 89 \mathrm{E}, 01.08 .2009,3$ 우 $0^{\top}, 4$ 우 우, leg. C. SchmidEgger (coll. S.-E.); Arapsuyu, Azmak, 10 m, 20.06.2002,

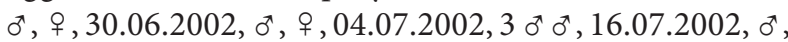
15.10.2003, ơ , leg. H. Özbek (Mentha longifolia); Çiğlik, 02.06.2009, ơ , leg. J.S. Ascher, H. Özbek (coll. AMNH); Demirtaş, $100 \mathrm{~m}, 29.07 .1985$, 우, leg. R. Hensen; Gündoğmuş, Glasandra, $1500 \mathrm{~m}, 36^{\circ} 83 \mathrm{~N} 32^{\circ} 05 \mathrm{E}$, 02.08.2009, o`, ㅇ, leg. C. Schmid-Egger (coll. S.-E.); Konyaaltı Beach, 2 m, 29.05.2009, đ, leg. J.S. Ascher, H. Özbek, J.G. Rozen (coll. AMNH); Manavgat, Lyrbe (Seleukeia), 07.06.1997, ơ, leg. H. Mühle. Edirne: Keşan, Mecidiye, 50 m, 29.07.2003, o, ㅇ , Ö. Çalmaşur (on Daucus carota). Erzurum: Atatürk University Campus, 2000 m, 06.08.1999, क, leg. R. Hayat (on Melilotus officinalis); Tortum, Derekap1, 20.09.2000, 우, leg. H. Özbek (on Eryngium sp.). Hakkâri: Beytüşşebap, Habur Deresi, o , 26.06.1985, leg. W. Schacht. Iğdır: Bayraktutan.

Material examined. Ardahan: Posof, Aşıkzülali, 1100 m, 25.07.2005, 2 ㅇ ㅇ, leg. C. Güçlü (on Eryngium creticum). Aksaray: Esmekaya, $38^{\circ} 16 \mathrm{~N} 33^{\circ} 22 \mathrm{E}$, 16.07.1998, ㅇ, leg. C. Schmid-Egger; Ihlara, $27 \mathrm{~km} \mathrm{NE}$, $38^{\circ} 14 \mathrm{~N} 34^{\circ} 18 \mathrm{E}, 18.07 .1998,20^{\top} 0^{\top}$, leg. C. Schmid-Egger. Artvin: Yusufeli, Demirkent, 1600 m, 01.09.1995, 우, leg. M. Kraus. Çorum: Mecitözü, 750 m, 05.06.2002, ㅇ, leg. W.H. Liebig. Erzurum: Atatürk University Campus, 2000 m, 09.08.1970, , leg. H. Özbek (on Melilotus officinalis); 03.09.1987, o* leg. R. Hayat; 11.07.2007, o*, ㅇ, leg. J.S. Ascher, H. Özbek, J.G. Rozen (coll. AMNH); 16.07.2003, 2 우, leg. J.G. Rozen and H. Özbek; $41 \mathrm{~km} \mathrm{~W}$ of Erzurum, Aşkale civarı, 02.07.2007, 2 o $^{\star} \mathrm{o}^{\star}$, leg. J.S. Ascher, H. Özbek, J.G. Rozen (coll. AMNH); Pasinler, 26.07.1997, क, leg. H. Özbek. Hakkâri: Yüksekova, 15 km E, 2100-2200 m, 20.07.1986, ơ, ㅇ, leg. A.W. Ebmer. Kayseri: Develi, Bakırdağı, 1700 m,

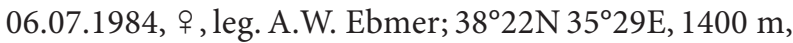
02.05.1985, ơ , leg. Aspöck/Rausch. Konya: Güneysınır, Gürağaç, 1020 m, 06.07.2000, $2 \sigma^{\star} \sigma^{\star}$, leg. M. Kesdek; Bozkır, Sarığlan, $1000 \mathrm{~m}, 25.07 .2003,2 \sigma^{\top} \sigma^{*}$, leg. M. Kesdek; Eflatun pinar1, 37051N 3137E, 15.07.1998, $30^{\top} 0^{\star}$, leg. C. Schmid-Egger. Muğla: Köyceyiz, 14.06.1987, o*, leg. P.H. van Doesburg. Nevşehir: Avanos, Zelve, 1060 m, 17.07.1984, ㅇ, leg. A.W. Ebmer. Niğde: $2 \mathrm{~km} \mathrm{S,} \mathrm{1250,} \mathrm{04.08.1984,} \mathrm{q,} \mathrm{leg.} \mathrm{A.W.} \mathrm{Ebmer;}$ Maden, Çiftehan, 1700-1800 m, 17.07.1990, o*, leg. A.W. Ebmer; Göreme, 38³9N 3453E, 17.07.1998, o, leg. C. Schmid-Egger. Şanlıurfa: Halfeti, 08.06.1983, o, leg. J. Schmidt. $890 \mathrm{~m}, 17.08 .2005$, o (M. officinalis); Melekli, 925 m, 16.08.2005, ơ, leg. H. Özbek. Isparta: Eğirdir, 06.06.1986, ㅇ, leg. Kadlec/Vorisek; Yalvaç,

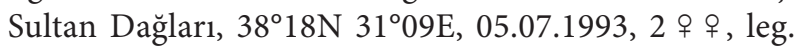
Jirousek (coll. Schwarz/Ansfelden). İzmir: Selçuk, 17.06.1968, ơ, leg. Arde. Konya: Beyşehir, 13.06.1966, 2 o o $^{\star}$, leg H.H.F. Hamann; Çumra, 1017 m, 13.08.2000, †, leg. M. Kesdek. Mersin: Silifke, Kizkalesi, 09.05.1998, , leg. N. Mohr. Muğla: Bodrum, Ägäis, Salmakıs, $37^{\circ} 02 \mathrm{~N} \quad 27^{\circ} 25 \mathrm{E}, \quad 13-25.07 .2001,70^{\star} \sigma^{\star}, 2$ 우 우, leg. F. Burger; Köyceğiz, 14.06.1987, đ^, leg. P.H. v Doesburg. Nevşehir: $2 \mathrm{~km} \mathrm{S,} 1250$ m, 04-19.07.1984, 2 주 o, 3 우 우, leg. A.W. Ebmer; Avanos 20 km N, 1200 m, 18.07.1984, $0^{\star}$, leg. A.W. Ebmer; Çardak, Acıgöl, 38³3N 34²47E, 07.07.1993, 5 우 우, leg. Halada/Jirousek (coll. Schwarz/

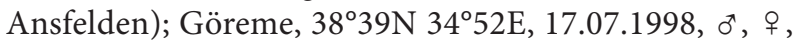
leg. C. Schmid-Egger; Zelve, 1050 m, 17.07.1984, o, leg. A.W. Ebmer. Niğde: Karakışlakçı, 1240 m, 07.07.1995, ○, ㅇ , leg. Y. Barbier (on Euphorbia altissima). Şanlıurfa: Halfeti, 400 m, 28.06.1987, क; 425 m, 07.08.1985, o, leg. R. Hensen. Şırnak: Beytüşşebap $19 \mathrm{~km} \mathrm{S,} 1200 \mathrm{~m}$, $37^{\circ} 24 \mathrm{~N} \quad 43^{\circ} 12 \mathrm{E}, \quad 26.06 .1985, \quad 26.06 .1985$, ㅇ, leg. M. Schwarz (coll. Schwarz/Ansfelden). 
Remarks: Eleven provinces are to be added to the distribution area of $H$. scutellatus. The species occurs at altitudes between sea level (Antalya, Edirne, İzmir) and $2000 \mathrm{~m}$ altitude (Erzurum). In general, it is a common species in both warmer and cooler regions, but more abundant in warmer regions. With the exception of the Black Sea region, it is reported from all geographical regions of Turkey. The flight period extends from May to the mid October. This long period indicates that it may have more than one generation. Flower visits are recorded at Daucus carota, Eryngium sp., Euphorbia altissima, Melilotus officinalis and Mentha longifolia. It is frequently observed in Turkey (19 provinces).

Distribution: Eastern Mediterranean, Caucasus, Azerbaijan, Syria, Iran. Turkey: Ankara, Denizli, Hatay, İzmir, Konya, Mersin, Osmaniye, Şanlıurfa (WARNCKE 1972); Adıyaman, Antalya, Edirne, Erzurum, Hakkâri, Iğdır, Isparta, Muğla, Nevşehir, Niğde, Şırnak.

\section{Hylaeus (Lambdopsis) tephronotus (WARNCKE, 1992)} Fig. 11

Prosopis (Lambdopsis) tephronota WARNCKE, 1992: 782-783, ㅇ. Turkey: Hakkâri.

Hylaeus (Lambdopsis) tephronotus (WARNCKE, 1992) - ITIS (2020)

Remarks: H. tephronotus is still exclusively known only from the nine females of the original type series; the male is unknown. The epithet is derived from $\tau \dot{\varepsilon} \varphi \rho a$ (Greek) and means "ash grey back", as for example are back and wing covers of the Persian Nuthatch (Sitta tephronota). The bee species in question is completely black. (Of course, scientific names do not necessarily have to agree with the facts.) Hylaeus tephronotus is clearly smaller than $H$. dilatatus, and resembles $H$. pfankuchi not only because of missing side spots. Different appears to be the clear dense fine punctation of the metasomal terga. A peculiarity is the strikingly long bristles on the lower part of the clypeus. H. tephronotus has been recorded as a mountain species from few sites (Hakkâri, 1700-3000 m), flying early in August. It is apparently a species of limited distribution, found only in Turkey. It is rarely recorded in Turkey (1 province, Fig. 17.8).

Distribution: Turkey: Hakkâri.

Subgenus Nesoprosopis PerkINs, 1899

\section{Hylaeus (Nesoprosopis) pectoralis FöRSTER, 1871}

Hylaeus pectoralis FöRSTER, 1871: 972-973, +. loc. typ. unknown [Germany or France].

Hylaeus (Nesoprosopis) pectoralis FöRster, 1871 Proshchalykin \& Dathe, 2012: 23.
Material examined: Erzurum: Oltu, Çamlıbel, $22 \mathrm{~km}$

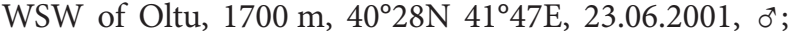
02.07.2001, +; 30.07.2003, + , leg. J.G. Rozen, H. Özbek (coll. AMNH). Muğla: Köyceğiz, 10.06.1987, ơ, leg. P. H. v. Doesburg.

Remarks: H. pectoralis is recorded in Turkey for the first time. The species is well known as a user of vacated galls of the chloropid fly Lipara lucens MeIgen in reed stems (Phragmites australis). It nests in the abandoned galls and is therefore confined to wetlands with stands of reeds. The collecting site in Erzurum is a narrow valley containing pastureland, through which a stream flows, a branch of the river Çoruh, beside which are small Phragmites patches.

In agreement with the general situation for this species, $H$. pectoralis shows a limited, disjunctive distribution in Turkey. Hitherto, H. pectoralis has been found at four places in Erzurum and Muğla only. It is rarely recorded in Turkey (2 provinces, Figs 16.7, 17.IV).

Distribution: Transpalaearctic from the Pyrenees to Japan, but because of its specific ecological requirements only locally recorded. Turkey: Erzurum and Muğla. New to the Turkish fauna.

Subgenus Paraprosopis Popov, 1939

\section{Hylaeus (Paraprosopis) clypearis (SCHENCK, 1853)}

Prosopis clypearis Schenck, 1853: 217, ơ. Germany: Nassau.

Hylaeus (Paraprosopis) clypearis (SCHENCK, 1853) - Aliev 1986: 265.

Material examined: Bursa: $225 \mathrm{~m}, 24.08 .1985,2 \sigma^{\star} \sigma^{*}$, 3 우 우, leg. R. Hensen; Çağlayan, 10-14.07.1997, 2 우 우, leg. Prudek/Riha; Mudanya, 25 m, 25.08.1985, o*, o , leg. R. Hensen. İstanbul: Boğaz (Bosfor), ?, o, leg. Hed. 32 (coll. RM Stockholm); Üsküdar, Anadolukavağ 1,30 m, 15.07.1987, $3 \sigma^{\star} \sigma^{\star}$, , , leg. P. van Ooijen. Yalova: Çiftlik, 50 m, 31.07.1986, ơ, leg. P. van Ooijen; Termal, 30 m, 28.08.1985, 우, leg. R. Hensen.

Remarks: According to Ascher \& Pickering (2019), H. clypearis is present in Turkey, but we could not identify the localities. Here we present data only from the Marmara region, to which the occurrence of $H$. clypearis is apparently restricted. The species is particularly abundant throughout the Mediterranean. It is collected only sparsely in Turkey (3 provinces).

Distribution: Western Palaearctic including North Africa. Turkey: Bursa, Yalova and İstanbul. 


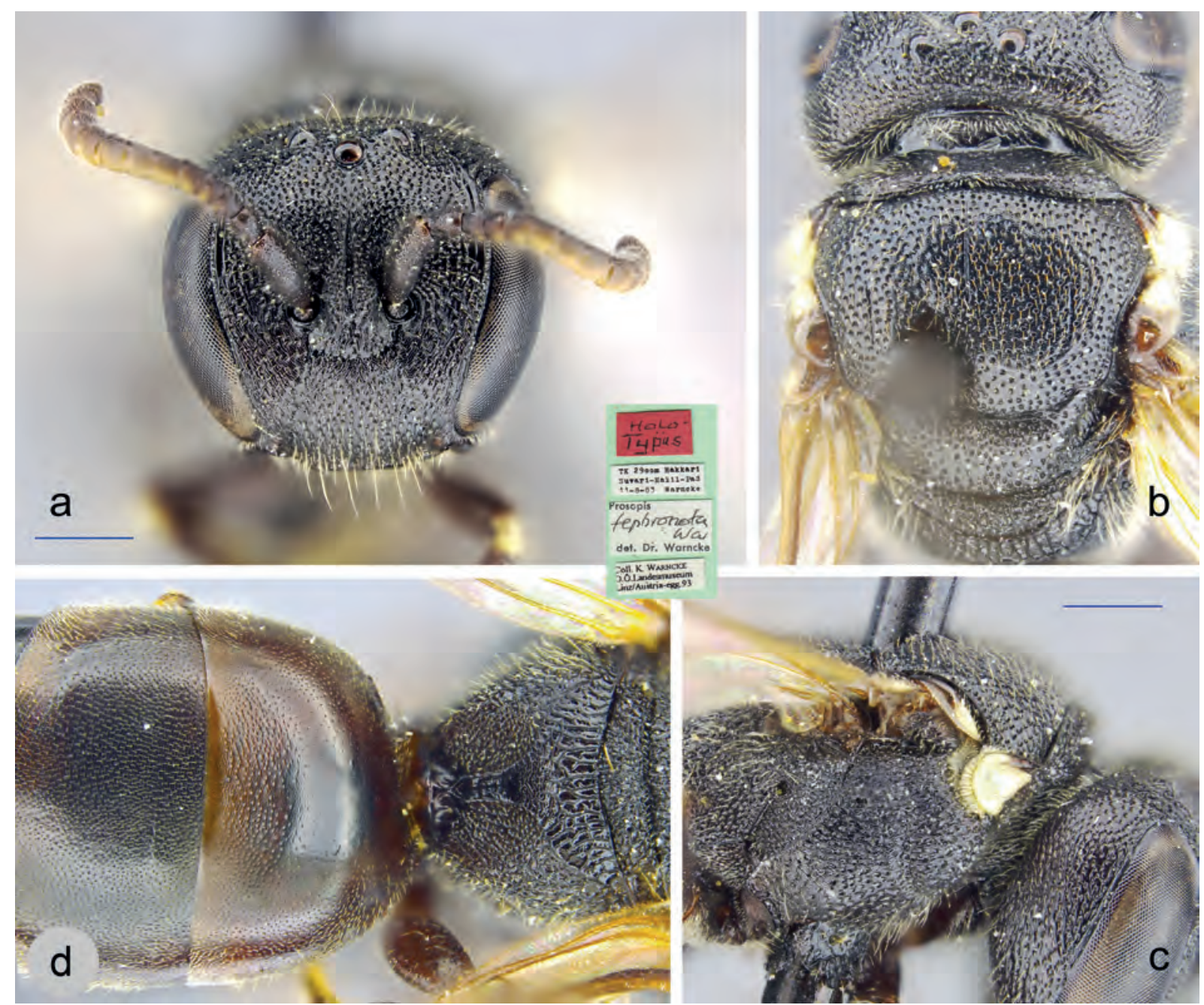

Fig. 11: H. (Lambdopsis) tephronotus (WARNCKe, 1992). Holotype female: a-face, b-mesonotum, c-mesopleuron, d-propodeum and metasoma. - Scale bar $0.5 \mathrm{~mm}$.

Hylaeus (Paraprosopis) decaocto (WARNCKE, 1992) Fig. 12

Prosopis (Paraprosopis) decaocta WARNCKE, 1992: 780-781, ㅇ․ Turkey: Hakkâri, Varegös.

Hylaeus (Paraprosopis) socheri DATHE, 2010: 61-63, 78. - syn. nov.

Hylaeus (Paraprosopis) decaocto (WARNCKE, 1992) - comb. nov.

Remarks: In $H$. decaocto, the yellow markings of the head (Fig. 12a,e) are amazingly similar to those of H. xanthopoda, but on the mesosoma, also scutellum and postscutellum are yellow. In contrast to H. xanthopoda, the integument of $H$. decaocto has distinct and very dense punctation, and the propodeum is rounded and without ridges. Dathe (2010) described the male and two females as H. (Paraprosopis) socheri from Iran, Province Yazd, at 2600-2900 m altitude.

The name "decaocta" (Greek $\delta \varepsilon \kappa a о \kappa \tau \dot{\omega}=$ eighteen) was coined without obvious relation to characters of this species: six yellow spots are present on the head, seven on the thorax, for example. As can be seen from the habitual usage of the author, Warncke explicitly applied this Greek numeral as latinized feminine adjective. According to the Latin grammar, numbers are adjectives which are dependent on the noun in declination and gender, so that Art. 31.2. of the ICZN applies.

$H$. decaocto was described on the basis of one female each from Hakkâri (1650 m, holotype) and Konya (1800 m). Since these sites are far away from each other, a wider distribution can be assumed, but possibly limited to the high mountain ranges. It is rarely collected in Turkey (2 provinces, Fig. 17.VI).

Distribution: Turkey: Hakkâri, Konya. Iran: Yazd province. 


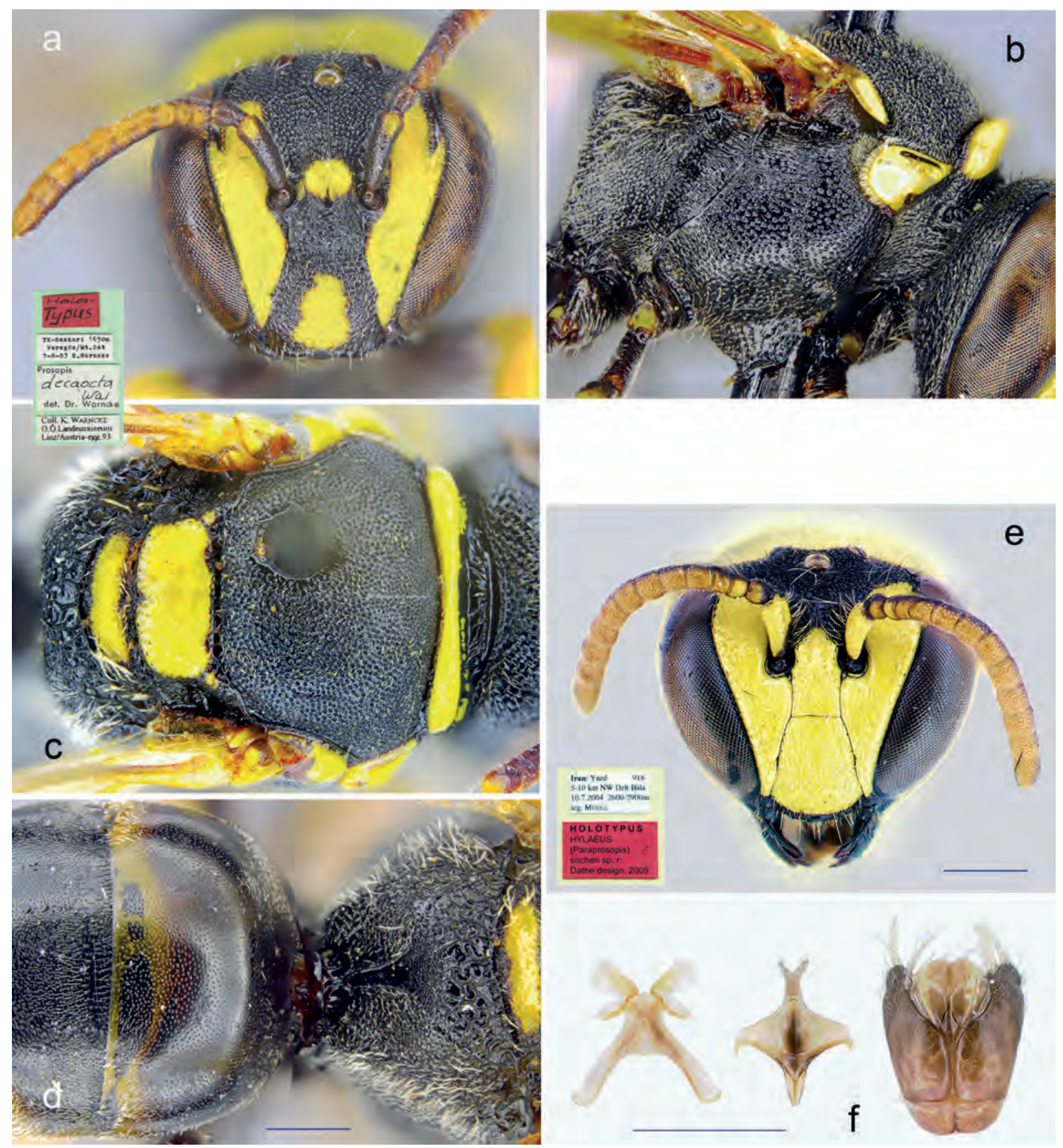

Fig. 12: H. (Paraprosopis) decaocto (WARncKe, 1992). Holotype female: a-face, b-mesopleuron, c-mesonotum, d-propodeum and metasoma. Male (holotype of $H$. socheri Dathe): e-face, f-terminalia. - Scale bar $0.5 \mathrm{~mm}$.

\section{Hylaeus (Paraprosopis) lineolatus (SCHEnCK, 1861)}

Prosopis lineolata Schenck, 1861:313, 323, 0. Germany: Nassau.

Prosopis (Paraprosopis) lineolata SCHENCK, 1861 - WARNCKe 1972: 766; 1981: 188; 1992: 758.

Hylaeus (Paraprosopis) lineolatus (Schenck, 1861) - Aliev 1986: 265.

Material examined: Adana: Pozant1, 06.07.1993, $20^{\text {त }} 0^{\text {, }}$, + , leg. J. Hladil. Aksaray: Kapadokya, Güzelyurt, $38^{\circ} 15 \mathrm{~N} 34^{\circ} 25 \mathrm{E}, 26.05 .2001$, $0^{\top}$, leg. W.H. Liebig. Ankara:
Kızılcahamam, 02.06.1986, 우, Kadlec/Vorisek. Antalya: $36^{\circ} 53 \mathrm{~N} 30^{\circ} 43 \mathrm{E}, 01-31.07 .1993$, $0^{*}$, + , leg. A. Puchner (coll. Schwarz/Ansfelden); $33 \mathrm{~km} \mathrm{NW}, 37^{\circ} 02 \mathrm{~N} 30^{\circ} 23 \mathrm{E}$, 23.07.1998, $60^{\top} 0^{\top}, 2$ ㅇ ㅇ , leg. C. Schmid-Egger (coll. E-G); $37^{\circ} 02 \mathrm{~N} 30^{\circ} 23 \mathrm{E}, 23.07 .1998,60^{\top} 0^{\top}, 2$ 우 우, leg. C. Schmid-

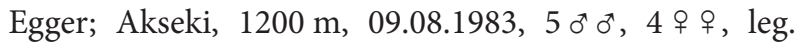
P. van Ooijen; Alanya, $20 \mathrm{~km} \mathrm{W,} 20 \mathrm{~m}, 07.08 .1985$, $30^{\top} 0^{7}$, leg. P. van Ooijen; Elmall, Beydağlari, Zedern, 1650-1750 m, 04.07.1990, $20^{\star} 0^{\star}$, 우, leg. A.W. Ebmer; İbadi $6 \mathrm{~km} \mathrm{SE}, 37^{\circ} 07 \mathrm{~N} 31^{\circ} 65 \mathrm{E}, 450 \mathrm{~m}, 31.07 .2009,40^{\top} 0^{\top}$, + , leg. C. Schmid-Egger; Güllük Dag1, $950 \mathrm{~m}, 36^{\circ} 59 \mathrm{~N}$ 030 $27 \mathrm{E}, 04.06 .1998,0^{*}$, ㅇ, leg. S.M. Blank; Terme- 
sos, 24.05.2009, ơ, ㅇ, leg. J. Ascher, J.R. Rozen, H. Özbek (coll. AMNH); Gündoğmuş, $1090 \mathrm{~m}$,

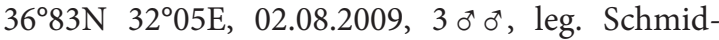
Egger (coll. E-G); Kemer, 01.05.1996, 2 ๙ $^{\star}$, leg. Sieber; Termassos, 24.05.2009, $30^{\star} 0^{\star}$, + , leg. J.S. Ascher, H. Özbek, J.G. Rozen (on Vitex agnus castus) (coll. AMNH); Akseki, Cevizli, 03.07.1996, , leg. Brechtel/Ehrmann. Artvin: Borçka, Ferhatlı, 21.V.2002, ơ, leg. J.G. Rozen, H. Özbek; Yusufeli, 700-900 m, 15-17.07.1995, o, 7 우 우, leg. Gelbrecht; Sarı̈öl, Kaçkar dağları, 1000 m, 17.07.1995, o, leg. Gelbrecht/Schwa. Balıkesir: Piribeyler, 39³8N $28^{\circ} 47 \mathrm{E}, 02.08 .1988$, o ${ }^{*}$, leg. Madl (coll. Schwarz/ Ansfelden). Bilecik: Osmaneli, 27.06.1983, 2 o $^{\star \top}$, ㅇ, leg. J. Hladil. Bingöl: $5 \mathrm{~km}$ E, 30.05.2002, ㅇ, leg. H. Özbek; Genç, 1000 m, 12.08.1985, †, leg. R. Hensen; Karlıova, Çobançantası, 1455 m, 10.08.2004, 2 우 우, leg. S. Çoruh. Bitlis: Ahlat, 1750 m, 15.08.1985, o, 2 우 우, R. Hensen; Tatvan, 1750 m, 16.08.1985, ơ, leg. R. Hensen. Burdur: 01.08.1998, $o^{\star}$, 우, I. Brunk; Çeltikçi, 02.09.1983, o*, 2 우 우, leg. J. Timmer. Bursa: $225 \mathrm{~m}, 24.08 .1985,2 \sigma^{\star} o^{\star}$, leg. R. Hensen; Armutlu, $50 \mathrm{~m}, 27.07 .1986$, ơ, leg. P. van Oojen; Orhaneli, 39 $54 \mathrm{~N} 28^{\circ} 59 \mathrm{E}, 02.08 .1988$, +, leg. Madl (coll. Schwarz/Ansfelden). Çorum: Osmancik, 900 m, 14.07.1985, + , leg. R. Hensen.

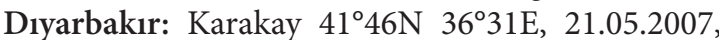
o , leg. W.H. Liebig. Edirne: Keşan, Mecidiye, 50 m, 22.07.2003, 2 ㅇ , leg. Ö. Çalmaşur. Erzurum: Oltu, Başakl1, 1700 m, 03.07.2001, ㅇ, o, leg. J.G. Rozen, H. Özbek; 24 km SW of Oltu, Başaklı, Ahırtap, 1550 m, 09.07.2007, 2 ㅇ , leg. J.S. Ascher, H. Özbek \& J.G. Rozen; Subatık, $1300 \mathrm{~m}, 13.08 .2004,2 \sigma^{\top} o^{\mathrm{T}}$; 30.08.2003, $7 o^{\star} \sigma^{\star}, 6$ ㅇ , leg. H. Özbek (on Melilotus officinalis); Tortum, Yeşilyurt, 2100 m, 30.08.2003, , leg. H. Özbek (on Eryngium creticum). Eskişehir: Sakari, Ilıca, Gümele, 06.07.1997, ơ, leg. Prudek/ Ríha. Gümüşhane: Torul, $1000 \mathrm{~m}, 40^{\circ} 34 \mathrm{~N} 39^{\circ} 17 \mathrm{E}$, 12.07.1985, ơ, 우, leg. M. Schwarz (coll. Schwarz/ Ansfelden). Hakkâri: Varagöz, $1750 \mathrm{~m}, 37^{\circ} 25 \mathrm{~N}$ $44^{\circ} 13 \mathrm{E}, 06.08 .1986,2$ ㅇ ㅇ , leg. S.M. Blank. Hatay: Samandağı, 06.07.1996, ơ, 5 우 ㅇ, leg. Brechtel/

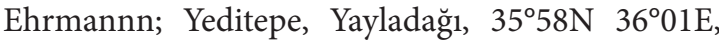
23.05.1987, o ${ }^{\star}$, leg. Madl (coll. Schwarz/Ansfelden); İskenderun, Belen, 28.08.1983, o ${ }^{\star}$, leg. J. Timmer. Isparta: Yalvaş, Sultan Dağları, $38^{\circ} 18 \mathrm{~N} 31^{\circ} 09 \mathrm{E}$, 05.07.1993, 주, 2 ㅇ 우, leg. Jirousek (coll. Schwarz/ Ansfelden). İzmir: Ödemiş, $1000 \mathrm{~m}, 38^{\circ} 22 \mathrm{~N} 27^{\circ} 58 \mathrm{E}$, 14.06.1985, o*, leg. Rausch. Kahramanmaraş: Alikayası Geçidi, 600 m, 09.07.1990, 2 o $^{\star}$, leg. A.W. Ebmer; Göksun, 1400 m, 26.06.1987, †, R. Hensen. Kayseri: Develi, Baakırdağ, Gezbeli Geçidi, 1700-1900 m, 06.07.1984, o, †, leg. A.W. Ebmer; Yeşilhisar: Araplı Geçidi, 1500 m, $38^{\circ} 15 \mathrm{~N} 35^{\circ} 07 \mathrm{E}, 03.06 .1985,0^{\star}$, leg. Aspöck, Rausch. Konya: $25 \mathrm{~km} \mathrm{~N}, 11.06 .1966$, ơ, 우, leg. HHF Hamann; Beyşehir, 1150 m, 18.06.1987, ơ , leg. R. Hensen; Bozkır, Sarığlan, 1000 m, 25.07.2003,
2 우 ㅇ , leg. M. Kesdek; Seydişehir, 03.07.1996, ㅇ, leg. Brechtel/ Ehrmann. Kütahya: Sobran, Porsuk Baraj1, 39 $40 \mathrm{~N} 30^{\circ} 10 \mathrm{E}$, 08.07.1993, 4 우 우, leg. Jirousek (coll. Schwarz/Ansfelden). Malatya: Doğanşehir, Reşadiye Geçidi, 12.07.1990, 1500 m, $\mathrm{o}^{\star}$, leg. A.W. Ebmer. Mardin: 1000 m, 02.07.1987, o`, leg. R. Hensen. Mersin: Mut, Sertavul Geçidi, 1550 m, 08.07.1990, $20^{\star} 0^{\star}$, leg. A.W. Ebmer; Silifke, Uzuncaburç, $400 \mathrm{~m}$, 02.08.1985, ơ , leg. R. Hensen; Kargican, 200 m, 04.08.1985, ㅇ, leg. R. Hensen. Muğla: Bodrum, Salmakız, $37^{\circ} 02 \mathrm{~N} 27^{\circ} 25 \mathrm{E}$, 13-25.07.2001, 4 o $^{\star}$, 4 우 우, leg. F. Burger; Köyceğiz, 50 m, 06.05.1998, $2 \mathrm{o}^{\star} \mathrm{o}^{\star}$, leg. G. Tozlu; Marmaris, Milas, $37^{\circ} 18 \mathrm{~N}$ 275ㅡ, 13.07.1990, $3 \sigma^{\star} o^{\star}$, leg. C. Schmid-Egger; Fethiye, Ölüdeniz, 50 m, 25.07.1986, 12 우, leg. P. van Ooijen. Muş: Buğlan Geçidi, 1650 m, 08.07.1984, o, leg. A.W. Ebmer. Nevşehir: Ürgüp, Topuzdağ 1 Geçidi, 1300 m, 17.07.1984, ơ, leg. A.W. Ebmer; Avanos, 38 $46 \mathrm{~N} 34^{\circ} 54 \mathrm{E}, 25.05 .2001$, ơ ${ }^{*}$ leg. W.H. Liebig. Niğde: Karakışlacı, 1240 m, 07.07.1995, 우, leg. Y. Barbier (on Euphorbia altissima). Sivas: Gürün, Şuğul Vadisi, 1400 m, 13.07.1990, 2 우 ㅇ leg. A.W. Ebmer. Şanlıurfa: Halfeti, 400 m, 28.06.1987, ơ, leg. R. Hensen. Şırnak: Betüşşebap $20 \mathrm{~km} \mathrm{~S}, 1200 \mathrm{~m}, 37^{\circ} 24 \mathrm{~N} 43^{\circ} 12 \mathrm{E}, 26.06 .1985$, ơ, + , leg.

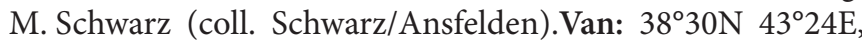
28.06.1993, o`, leg. M. Halada (coll. Schwarz/Ansfelden). Yalova: Termal, $30 \mathrm{~m}, 28.08 .1985$, o ${ }^{\star}$, leg. R. Hensen. Yozgat:

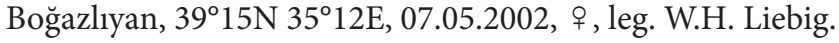

Remarks: With a further thirty provinces, our knowledge of the distribution area of $H$. lineolatus is significantly enlarged. The available records show that the species is one of the most abundant and widespread in Turkey from sea level to $2100 \mathrm{~m}$ altitude; it lives in various biotopes, mostly in open areas. The flight season extends from the beginning of May to the end of August, with a peak in July. Eryngium creticum, Euphorbia altissima, Melilotus officinalis and Vitex agnus-castus have been observed as food plants. It is frequently recorded in Turkey (41 provinces).

Distribution: Western Palaearctic. In Turkey: Adana, Ankara, Çanakkale, Denizli, Erzurum, Hatay, İzmir, Konya, Kütahya, Mersin, Osmaniye (WARncke 1972); Aksaray, Antalya, Artvin, Balıkesir, Bilecik, Bingöl, Bitlis, Burdur, Bursa, Çorum, Diyarbakır, Edirne, Eskişehir, Gümüşhane, Hakkâri, Isparta, Kahramanmaraş, Kayseri, Malatya, Mardin, Muğla, Muş, Nevşehir, Niğde, Şanlıurfa, Şırnak, Sivas, Van, Yalova, Yozgat.

\section{Hylaeus (Paraprosopis) pictipes NyLANDER, 1852}

Hylaeus pictipes Nylander, 1852: 95-97, ․ South Sweden.

Prosopis (Paraprosopis) pictipes pictipes (Nylander, 1852) WARnCKe 1972: 749.

Hylaeus (Paraprosopis) pictipes Nylander, 1852.

Material examined: Erzurum: Oltu, Başaklı, $1700 \mathrm{~m}$, 12.08.1979, o`, leg. H. Özbek; 01.08.1977, o`, leg. H. Özbek; Palandöken, $2300 \mathrm{~m}, 16.08 .1987$, †, leg. H. Özbek (on Eryngium sp.). Sinop: 17.07.1979, ㅇ, leg. H. Özbek (Onopordum sp.). 
Remarks: The provinces Erzurum and Sinop are added to the distribution lists of $H$. pictipes. All available distribution data, including those listed here, must be further verified. The data of WARNCKE (1972 ff.) mix the actual species $H$. pictipes with a number of different taxa, especially with $H$. taeniolatus. The latter species is widespread and very common throughout the Mediterranean. H. pictipes is collected sparsely in Turkey (7 provinces).

Distribution: Northern parts of Europe east to Ukraine. Introduced to North America. In Turkey: Antalya, Bursa, Hatay, Mersin, Tokat (WARNCKe 1972); Erzurum, Sinop.

\section{Hylaeus (Paraprosopis) sinuatus (Schenck, 1853)}

Prosopis sinuata Schenck, 1853: 216, ơ (nec 우). Germany: Nassau.

Hylaeus (Paraprosopis) sinuatus (Schenck, 1853) - Aliev 1986: 265.

Material examined: Aksaray: Sivrihisar Geçidi, $1800 \mathrm{~m}$, $38^{\circ} 16 \mathrm{~N} 34^{\circ} 19 \mathrm{E}, 03.07 .1984$, ㅇ, leg. A.W. Ebmer. Artvin: Borçka, Ferhatll, 800 m, 21.05.2002, ơ , leg. J.G. Rozen, H. Özbek; Murgul, Damar, 01.07.1997, 3 đ $^{\top} 0^{\top}$, + , leg. Brudek/Riha. Bursa: Mudanya, $25 \mathrm{~m}, 25.08 .1985$, ơ, 4 우 오, leg. R. Hensen. Erzurum: 1900 m, 13.07.2004, ${ }^{*}$, leg. H. Özbek; Atatürk Universitty research field 6 nolu kuyu, 20.06.1976, ๆ, leg. H. Özbek (on Onobrychis viciifolia); 12.08.1970, ơ leg. H. Özbek (on Melilotus officinalis); 01.06.1974, ơ , leg. H. Özbek (on Salix sp.); Çat yolu, DSI Göleti, 3947N 41 ${ }^{\circ} 09 \mathrm{E}, 09.07 .2004$, $\mathrm{o}^{7}$, leg. H. Özbek, J.G. Rozen; Oltu, Başakl1, 1600 m, 12.08.1979, ㅇ, 3 o $^{\top}{ }^{\top}$, leg. H. Özbek (on Daucus carota);

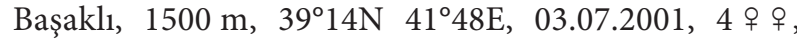
$30^{\top} o^{\star}$, leg. H. Özbek, J.G. Rozen; Başakll, Ahırtap, 09.07.2007, 2 o $^{\star}$ o $^{\star}$, leg. J.S. Ascher, H. Özbek, J.G. Rozen; Pasinler, Demirdöven Baraj1, $1800 \mathrm{~m}$, 01.07.2007, $\mathrm{o}^{\top}$, leg. J.S. Ascher, H. Özbek, J.G. Rozen; Tortum, 1700 m, 16.07.1987, 3 우, leg. R. Hensen. Hakkâri: Yüksekova $15 \mathrm{~km} \mathrm{E,} 2100-2200 \mathrm{~m}, 20.07 .1986,2$ 우, leg. A.W. Ebmer. Mersin: Mut, Dereköyü, 02.09.1987, $2 \sigma^{A} \sigma^{A}$, leg. H. Özbek (on D. carota). Nevșehir: Ürgüp, Topuzdağ 1 geçidi, $1300 \mathrm{~m}, 17.07 .1984,0^{\star}$, + , leg. A.W. Ebmer. Sivas: Gürün, 1200 m, 24.07.1986, ㅇ, leg. S.M. Blank.

Remarks: Six provinces can be added here to the known distribution records of $H$. sinuatus. In determining this species, attention must be paid to possible confusion with H. soror. H. sinuatus is a common species found both in warmer (Bursa, Mersin) and cooler regions (Erzurum, Hakkâri). The flight period is from May to the end of August. Visits were observed to flowers of Daucus carota, Melilotus officinalis, Onobrychis viciifolia and Salix sp. The species is frequently recorded in Turkey (20 provinces).
Distribution: All of Europe, Russia, Azerbaijan, Georgia, Cyprus, Iran. Turkey: Amasya, Ankara, Balıkesir, Bilecik, Bursa, Denizli, Hatay, İstanbul, Konya, Kütahya, Mardin, Nevşehir, Şanlıurfa (WARnCKe 1972); Kars (Özвек 1977); Aksaray, Artvin, Erzurum, Hakkâri, Mersin and Sivas.

\section{Hylaeus (Paraprosopis) soror (PÉREZ, 1903)}

Prosopis soror PÉREz, 1903: 233, ơ. Italy: Sicily.

Prosopis (Paraprosopis) sinuata gribodoi VACHAL, 1895 WARNCKE 1972: 751.

Hylaeus (Paraprosopis) soror (PÉREZ, 1903).

Material examined: Adıyaman: 20 km E Gölbaşı, 600 m, $37^{\circ} 44 \mathrm{~N} 37^{\circ} 55 \mathrm{E}, 12.05 .2002$, ơ , leg. J.G. Rozen, H. Özbek (coll. AMNH); Nemrut Dağ 1 , Karadut, $37^{\circ} 56 \mathrm{~N} 38^{\circ} 47 \mathrm{E}$, 02.07.1993, ơ, leg. M. Halada (coll. Schwarz/Ansfelden). Ağrı: $1700 \mathrm{~m}, 39^{\circ} 44 \mathrm{~N} 43^{\circ} 03,27.06 .1993,360^{\top} 0^{\star}, 4$ ㅇ 우, leg. M. Halada (coll. Schwarz/Ansfelden); Eleşkirt, 2200 m, 14.07.1987, o , leg. R. Hensen. Aksaray: Ihlara, $38^{\circ} 14 \mathrm{~N}$ $34^{\circ} 18 \mathrm{E}, 18.07 .1998$, $0^{\star}$, leg. C. Schmid-Egger. Ankara: 10.06.1973, ơ, leg. Warncke/RMS (coll. RM Stockholm). Antalya: Gündoğmuş, Gelesandra $1500 \mathrm{~m}, 36^{\circ} 83 \mathrm{~N}$ $32^{\circ \circ} 05 \mathrm{E}, 02.08 .2009$, + , leg. C. Schmid-Egger; Akseki, $1200 \mathrm{~m}, 09.08 .1985,4 \sigma^{\star} 0^{\top}$, leg. P. van Ooijen. Bingöl: Genç, 1500 m, 12.08.1985, 3 o $^{\star} \sigma^{\star}$, leg. R. Hensen; Genç $15 \mathrm{~km} \mathrm{~S}, 1400 \mathrm{~m}, 13.08 .1985$, o o , leg. R. Hensen. Burdur: Çeltikçi, 02.09.1983, 4 o $^{\star} 0^{\star}$, leg. J. Timmer. Bursa: $225 \mathrm{~m}$, 24.08.1985, 2 o $^{\top} \mathrm{o}^{\top}$, leg. R. Hensen; Kurşunlu, 15.07.1997, $\sigma^{7}$, leg. Prudek/Riha. Dıyarbakır: Çınar, $850 \mathrm{~m}$, 10.08.1985, $10 \sigma^{\star} \sigma^{*}$, leg. R. Hensen. Erzurum: Horasan, Aras Vadisi, $1650 \mathrm{~m}, 16.06 .1973,0^{7}$, + , leg. Warncke/ RMS (coll. RM Stockholm); Oltu, Başakll, 18 km WSW of Oltu, $1500 \mathrm{~m}, 29^{\circ} 14 \mathrm{~N} 41^{\circ} 48 \mathrm{E}, 27.06 .2001$, 우 , leg. J.G. Rozen, H. Özbek (coll. AMNH); Çamlıbel, 1600 m, 26.07.2000, ơ ; İnanmış, $1700 \mathrm{~m}, 26.07 .2000$, ơ (on Marrubium parviflorum); Subatık, 1300 m, 13.08.2004, $\sigma^{7}$, leg. H. Özbek. Hakkâri: Yüksekova 15 km E, 2100$2200 \mathrm{~m}, 20.07 .1986,0{ }^{\top}, 2$ ㅇ ㅇ, $20 \mathrm{~km} \mathrm{E}, 23.07 .1986$, $0^{7}, 4$ ㅇ 우 , leg. A.W. Ebmer. Kahramanmaraş: Göksun, Salyan-Ahmetçik arası, 10.07.1990, ơ , leg. AW. Ebmer; Göksun, 1400 m, 26.06.1987, 4 o $^{\top} \sigma^{\star}$, leg. R. Hensen. Kars: Sarıkamış, Karakurt, TCK Çeşmesi, $1501 \mathrm{~m}$, $40^{\circ} 08 \mathrm{~N} 42^{\circ} 21 \mathrm{E}, 09.07 .2003$, + , leg. H. Özbek; Akkurt, Çeşme Üzeri, 1550 m, 19.06.2004, 2 우 ㅇ, leg. H. Özbek. Kayseri: Develi, Bakırdağı, 1700 m, 06.07.1984, $20^{\top} \sigma^{*}$, leg. A.W. Ebmer. Kırșehir: Kulpak, $38^{\circ} 27 \mathrm{~N} 34^{\circ} 14 \mathrm{E}$, 28.05.2001, ơ , leg. W.H. Liebig. Konya: Bozkır, Sarıoğlan, 1000 m, 25.07.2003, 5 우 ㅇ, leg. M. Kesdek; Güneysınır, Karagüney, 03.09.2000, ơ , leg. Kesdek. Kütahya: Sobran,

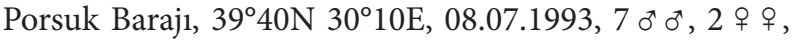
leg. Jirousek (coll. Schwarz/Ansfelden). Malatya: Karahan Geçidi, 1800 m, 07.07.1984, ㅇ, leg. A.W. Ebmer. Mardin: 1000 m, 02.07.1987, 2 ㅇ 우, leg. Hensen; Midyat $1000 \mathrm{~m}, 03.07 .1987, \mathrm{o}^{\top}, 4$ 우 웅 leg. Hensen; $37^{\circ} 25 \mathrm{~N}$ $41^{\circ} 22 \mathrm{E}, 25.05 .1983,3$ 우 9 , leg. W. Schacht (coll. Schwarz/ 
Ansfelden). Mersin: Haspinarı, 1640 m, 04.07.1995, $0^{*}$, leg. Y. Barbier (on Euphorbia kostschyana). Muğla: Bodrum, Salmakis, $37^{\circ} 02 \mathrm{~N} 27^{\circ} 25 \mathrm{E}, 21-25.07 .2001$, ơ, leg. F. Burger (on Polygonum sp.); Karaova E, 25.07.2002, $2 \sigma^{\star} o^{\star}$, leg. E. Kwast; Nevşehir: Acıgöl, Çardak, 38³3N 34 47E, 07.07.1993, 3 o $^{\top}$ o $^{\star}$, M. Halada; Avanos, Zilve, $1050 \mathrm{~m}, 18.08 .1984$, ㅇ, leg. A.W. Ebmer; $1000 \mathrm{~m}$, 22.06.1987, ơ , leg. Hensen; Kaymaklı, 1200 m, 23.06.1987, $2 \sigma^{\top} \sigma^{\star}$, leg. Hensen; Ürgüp, $1100 \mathrm{~m}, 11.08 .1985,2 \sigma^{\star} \sigma^{\star}$, leg. P. van Ooijen. Niğde: Karakışlakçı, 1240 m, 07.07.1995, + , leg. Y. Barbier (on Euphorbia altissima). Şırnak:

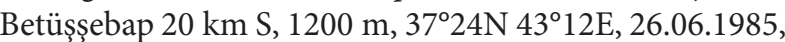
$0^{*}$, ㅇ, leg. M. Schwarz. Tunceli: $960 \mathrm{~m}, 13.06 .1973$, ㅇ , leg. Warncke/RMS (coll. RM Stockholm). Uşak: Sivasl1, 830 m, $38^{\circ} 37 \mathrm{~N} 29^{\circ} 35 \mathrm{E}, 21.05 .1983$, ơ ${ }^{\star}$, ㅇ, leg. H. Hüttinger. Van: $10 \mathrm{~km} \mathrm{~S}, 2200 \mathrm{~m}, 18.07 .1986$, ㅇ, A.W. Ebmer; Merkez, $38^{\circ} 30 \mathrm{~N} 43^{\circ} 24 \mathrm{E}, 26.06 .1993$, 우 , leg. M. Halada. Yalova:

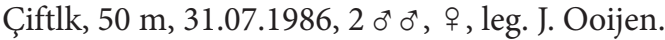

Remarks: H. soror is recorded almost throughout the country, preferring areas with a continental climate at altitudes from $600 \mathrm{~m}$ to $2200 \mathrm{~m}$ (Hakkâri). It can be considered as an inhabitant of warm and dry habitat types living in steppes and open areas, sometimes even in cool and mountainous environments. The flight season extends from late May to early September with a peak in July. Flower visits were observed at Euphorbia altissima, Marrubium parviflorum and Polygonum sp. It is frequently collected in Turkey (28 provinces).

Distribution: Western Palaearctic. Turkey: Adiyaman, Ağrı, Aksaray, Ankara, Antalya, Bingöl, Burdur, Bursa, Diyarbakır, Erzurum, Hakkâri, Kahramanmaraş, Kars, Kayseri, Kırșehir, Konya, Kütahya, Malatya, Mardin, Mersin, Muğla, Nevşehir, Niğde, Şırnak, Tunceli, Uşak, Van, Yalova.

The papers by WARNCKE (1972 ff.) provide no information, because he treated this species as a synonym of H. sinuatus.

\section{Hylaeus (Paraprosopis) styriacus FöRSTER, 1871}

Hylaeus styriacus FöRSTER, 1871: 1062-1064 ơ. Austria: Steiermark.

Prosopis (Nesoprosopis) styriaca (FöRSTER, 1871) - WARNCKE 1972: 765.

Hylaeus (Paraprosopis) styriacus FöRster, 1871 - Aliev 1986: 265.

Prosopis (Paraprosopis) styriaca crecca WARNCKE, 1992: 776-777, ㅇ ơ. Turkey - syn. nov.

Material examined: Ağrı: $1700 \mathrm{~m}, 39^{\circ} 44 \mathrm{~N} 43^{\circ} 03 \mathrm{E}$, 27.06.1993, ơ , leg. Jirousek (coll. Schwarz/Ansfelden); Eleşkirt, 30 km W, 2200 m, 14.07.1987, ơ, leg. R. Hensen. Antalya: Altıkaya, Köprülü Milli Parrkı, 954 m, 37²13N $31^{\circ} 07 \mathrm{E}, 13.06 .2002$, o ${ }^{\star}$, leg. G. Degen (coll. S.-E.); Güllük

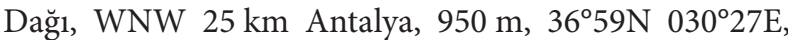

04.06.1998, đ`, leg. S.M. Blank. Hakkâri: Varagöz, 37²5N

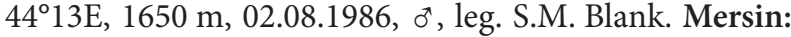
Erdemli $20 \mathrm{~km} \mathrm{NW,} 900$ m, 05.07.1996, ㅇ, leg. Tyrner/ Vorisek. Nevşehir: Ürgüp, Topuzdağı geçidi, 1300 m, 17.07.1984, ơ, leg. A.W. Ebmer.

Remarks: Three provinces are added to the recorded distribution of $H$. styriacus. The region of Eastern Anatolia is newly added. The species is sporadically distributed. It lives in the Mediterranean area at 900-2200 m altitude and can be considered to be a mountain species. The flight period is from June to August. It is collected only sparsely in Turkey (6 provinces).

Distribution: Europe, concentrated in the eastern area; western Asia including Armenia, Azerbaijan, Lebanon and Iran. In Turkey: Kastamonu, Mersin (WARNCKE 1972); Antalya, Mersin (as Prosopis styriaca crecca WARNCKe, 1992); Ağrı, Hakkâri, Nevşehir.

\section{Hylaeus (Paraprosopis) taeniolatus FönSTER, 1871}

Hylaeus taeniolatus Förster, 1871: 1068-1069, ㅇ․ Italy: Sicily. Prosopis (Paraprosopis) pictipes pictipes (Nylander, 1852) WARNCKE 1972: 749; 1992: 756.

Hylaeus (Paraprosopis) diplonymus (SchulZ, 1906) - DATHE 1980: 248.

Hylaeus (Paraprosopis) taeniolatus FöRSTER, 1871.

Material examined: Adana: Aladağ, 780 m, 02.07.1995, ㅇ, leg. Y. Barbier (on Hippomarathrum microcarpum) (coll. Schwarz/Ansfelden). Antalya: WNW $25 \mathrm{~km}$, Güllük Dağ 1,950 m, 3659N 030²7E, 04.06.1998, $20^{\star} o^{\top}$, +, leg. S.M. Blank; Arapsuyu, Azmak, 5 m, 20.06.2002,

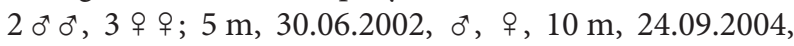
$0^{\star}$, + , leg. H. Özbek (Mentha longifolia); Kemer, 7 km W, Ovacık Köyü, Kesme Boğazı, 130 m, 36³6N 030²9E, 05.06.1998, 15 우 우, leg. C. Kutzscher; 05.06.1998, 18 o $^{\top} o^{\top}$, 2 우 우, leg. S.M. Blank (on Paliurus spina christi); 36 $36 \mathrm{~N}$ $030^{\circ} 29 \mathrm{E}, \sigma^{\star}$, + , leg. Lange/Ziegler; Konyaaltı Beach, $3 \mathrm{~m}, 29.05 .2009,2 o^{\star} o^{*}$, ㅇ, leg. J.S. Ascher, H. Özbek, J.G. Rozen (coll. AMNH); Termessos, 24.V.2009, $5 o^{\star} \sigma^{\star}$, leg. J.S. Ascher, H. Özbek (coll. AMNH). Bitlis: Nemrut Dağı, 2000 m, 16.08.1998, ơ, leg. M. Halada/ZSSM. Bursa: Kurşunlu, 15.07.1997, ơ, ㅇ, leg. Prudek/Richa. Hakkâri: Oramar 10 km E, 1700 m, 29.06.1985, 우, leg M. Schwarz (coll. Schwarz/Ansfelden); Hatay: Samandağ 1 $30 \mathrm{~km} \mathrm{~N}$, Nur dağları, 06.07.1996, 4 ㅇ 우, leg. Brechtel/ Ehrmann; $10 \mathrm{~km} \mathrm{E,} \mathrm{Asi} \mathrm{Nehri,} \mathrm{05.07.1998,} 3$ ㅇ 우, leg.

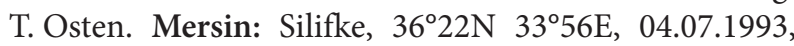
$3{\sigma^{\top} \sigma^{\star}}^{*}$, leg. M. Halada (coll. Schwarz/Ansfelden). Muğla:

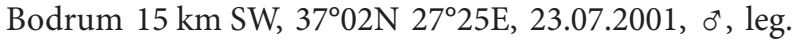
F. Burger; Salmakıs, $37^{\circ} 02 \mathrm{~N}$ 27 $25 \mathrm{E}, 16-17.07 .2001$, $3 \sigma^{\star} o^{\star}$, leg. F. Burger (coll. Schwarz/Ansfelden).

Remarks: In general, the distribution of $H$. taeniolatus is limited to the coastal area of the Mediterranean 
region from Hatay to Muğla. Interestingly it is also reported from the mountains of Bitlis and Hakkâri (up to $2000 \mathrm{~m}$ ). The flight time is from May to the end of September. Recorded flower visits are at Hippomarathrum microcarpum, Mentha longifolia, Paliurus spina christi. It is collected moderately in Turkey (8 provinces).

Distribution: Western Palaearctic, mainly Mediterranean and Central Europe. In Turkey: Adana, Antalya, Bitlis, Bursa, Hakkâri, Hatay, Mersin, Muğla.

The papers by WARNCKe (1972 ff.) provide no information, because he treated this species as a synonym of H. pictipes.

Subgenus Patagiata BLÜTHGEN, 1949

\section{Hylaeus (Patagiata) cervinus (WARNCKE, 1992)}

Prosopis (Prosopis) cervina WARNCKe, 1992: 794-795, 웅. Turkey: Horasan, Aras valley; Iran(?).

Hylaeus (Hylaeus) cervinus (WARNCKe, 1992) - DATHE 2000: 172-173.

Hylaeus (Patagiata) cervinus (WARNCKE, 1992) - ITIS (2020).

Remarks: WARNCKE (1992) described H. cervinus from Erzurum (loc. typ.) and Kars. Later DATHE (2000) redescribed this species and added another specimen from Adana. Despite years of intensive collecting, this species unfortunately could not been found again in these provinces, or anywhere else in the country. However, based on the few available data, it cannot be said whether the species is threatened or extinct. From Turkey, it is only sparsely known (3 provinces).

Distribution: Turkey: Adana, Erzurum, Kars (WARNCKE 1992; DATHE 2000: 172). The allocation of a single female from Iran to this taxon is questionable (WARNCKE 1992: 794).

\section{Hylaeus (Patagiata) difformis (Eversmann, 1852)}

Prosopis difformis EversmanN, 1852: 52, ơ ㅇ․ Russia: Orenburg Province.

Prosopis (Prosopis) difformis difformis Eversmann, 1852 WARNCKE 1972: 756; 1992: 763.

Hylaeus (Hylaeus) difformis (Eversmann, 1852) - Michener 2007: 208; CHEN \& Xu 2009: 47.

Hylaeus (Patagiata) difformis (Eversmann, 1852) - Aliev 1986: 267; Proshchaly kin \& Dathe 2012: 24.

Material examined: Antalya: Beydağları, Sineklibeli geçidi, 1550 m, 05.07.1990, ㅇ, leg. A.W. Ebmer. Artvin: Yusufeli, Kaçkar dağı, 1300 m, 16-19.07.1995, ㅇ, leg. Gelbrecht/Schwabe. Erzurum: Tortum, Kirmalı, 2500 m, 03.08.2004, $2 \sigma^{\star} \sigma^{\star}$, leg. S. Çoruh. Hakkâri: Varegöz, Sat

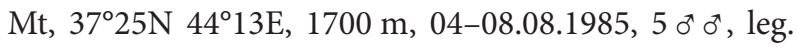
W. Schacht (coll. Schwarz/Ansfelden).

Remarks: Although $H$. difformis has a large distribution area outside Turkey, it was previously only known from the province Mersin. In the present study it is newly recorded from four provinces, which also represent new records for the Black Sea and Eastern Anatolia regions. The species has a sporadic distribution and occurs from $1000 \mathrm{~m}$ to $2500 \mathrm{~m}$. In Turkey it is collected only sparsely (5 provinces).

Distribution: Transpalaearctic, from the Iberian Peninsula to the Russian Far East. Turkey: Mersin (WARnCKE 1972); Antalya, Artvin, Erzurum, Hakkâri.

\section{Subgenus Prosopis FabRICIUS, 1804}

\section{Hylaeus (Prosopis) confusus NyLANDER, 1852}

Hylaeus confusus Nylander, 1852: 232, o o ơ. Europe.

Prosopis (Nesoprosopis) gibba confusa (NyLANDER, 1852) WARNCKe 1972: 759-760; 1992: 748-750.

Hylaeus (Prosopis) confusus Nylander, 1852 - Aliev 1986: 262. Straka \& Bogusch 2011: 52-61; Dathe \& ProshchALYKIN 2017: 375; 2018: 71.

Material examined: Ardahan: Çamlıçatak, $2300 \mathrm{~m}$, 10.08.1976, 우, ơ, leg. H. Özbek (on Carduus nutans); Posof, Aşızzülali, $1100 \mathrm{~m}, 25.07 .2005,2 \sigma^{\star} \sigma^{\star}$, †, leg. C. Güçlü. Artvin: Murgul, Damar, 01.07.1997, o*, leg. Prudek, Riha. Bolu: lake env., 4044N, 31³7E, 21.06.1993, , leg. M. Halada. Bursa: National park, $40^{\circ} 07 \mathrm{~N} 29^{\circ} 11 \mathrm{E}$, $1700 \mathrm{~m}, 01.08 .1988$, ㅇ, leg. Madl. Erzincan: Refahiye, $8 \mathrm{~km} \mathrm{~N}, 1700 \mathrm{~m}, 14.07 .1984$, , leg. A.W. Ebmer. Erzurum: Atatürk Üniversity research field, $2000 \mathrm{~m}$, 05.09.1966, ơ, leg. H. Özbek (on Onobrychis viciifolia); Ilıca, Ağzıaçık geçidi, 2000 m, 19.07.2003, 2 ㅇ + , leg. H. Özbek (on Cephalaria procera). Rize: İkizdere, Ovit Pass, 1500 m, 11.07.1985, ㅇ, leg. W. Schacht.

Remarks: H. confusus is widespread and often common. In the study by STRAKA \& Bogusch (2011), some closely related species of a " $H$. gibbus group" were better defined, although the complete group was not covered. In Turkey $H$. confusus appears as a lower mountain species collected at 1100-2300 m altitude. Our findings support observations from Southern Europe, where H. confusus is to be found mainly in mountain regions. At present it is considered in Turkey to be sparsely collected (6 provinces).

Distribution: Transpalaearctic to Kuril Islands, including Central Asia, Mongolia, China, Japan (Proshchalykin \& DAthe 2017); introduced to America (Costa Rica, Maryland). Turkey: Ardahan, Artvin, Bolu, Bursa, Erzincan, Rize. 


\section{Hylaeus (Prosopis) damascenus (Magretti, 1890)}

Prosopis damascena Magretti, 1890: 537-538, 우 ơ. Syria: Damascus.

Prosopis (Nesoprosopis) gibba damascena MAgRetti, 1890 -

WARNCKE 1972: 760; 1981: 185; 1985: 58; 1992: 750.

Hylaeus (Prosopis) damascenus (MAGretti, 1890) - ITIS (2020).

Remarks: H. damascenus is known from Syria, Lebanon, Iraq and Iran. The data for Turkey go back to WARNCKE (1981, 1985), who indicates this species for SE Turkey (1981: 185) or "southern border of Turkey" (1985: 59) respectively. More detailed collecting data were not provided. Up to now there have been no further findings, but the occurrence of the species in Turkey is likely.

Distribution: Syria, Lebanon, Iraq, Iran, Turkey.

\section{Hylaeus (Prosopis) duckei (AlFKen, 1905)}

Prosopis duckei Alfken, 1904: 119, o ơ. Italy: Trieste.

Prosopis (Nesoprosopis) duckei Alfken, 1904 - WARNCKe 1972: 765; 1992: 750.

Hylaeus (Prosopis) duckei (Alfken, 1905) - Aliev 1986: 262.

Material examined: Afyonkarahisar: Şuhut, $1184 \mathrm{~m}$, 23.05.2009, ơ, leg. J.G. Rozen, J.S. Ascher, H. Özbek. Antalya: Kocaaliler, Karacaören Barj1, 23.05.2009, o*, leg. J.S. Ascher, H. Özbek, J.G. Rozen (coll. AMNH); Termassos, $37^{\circ} 02 \mathrm{~N} 30^{\circ} 28 \mathrm{E}, 500 \mathrm{~m}, 25.05 .1985,2$ 우 우, leg. Rausch. Hakkâri: Yüksekova, Varegöz, Sat Dağı, 1700 m, $37^{\circ} 25 \mathrm{~N} 44^{\circ} 13 \mathrm{E}, 04-08.08 .1983,3$ 우 잉 leg. W. Schacht (coll. Schwarz/Ansfelden). Mersin: Balandiz, $36^{\circ} 20 \mathrm{~N}$ $33^{\circ} 46 \mathrm{E}, 1000 \mathrm{~m}, 06.06 .1985$, ơ , Aspöck/Rausch. Van: Gevaş, Göllü, 1700 m, 38 $43 \mathrm{~N} 43^{\circ} 19 \mathrm{E}, 23.06 .1993,5 \diamond^{\star} o^{*}$, 5 우 ㅇ, leg. M. Halada (coll. Schwarz/Ansfelden).

Remarks: H.duckei is documented here from four further provinces. The species has a sporadic distribution and is moderately collected in Turkey (8 provinces). - We regard the taxon Prosopis duckei stellata WARNCKE, 1992 as a separate species (see below).

Distribution: Southern Europe and Turkey. In Turkey: Niğde (WARNCKE 1972); Afyonkarahisar, Antalya, Mersin, Van.

\section{Hylaeus (Prosopis) excelsus (AlfKen, 1935)}

Prosopis excelsa AlfKen, 1935: 23-24, ․ Iran: Asherabad (Elburs).

Prosopis (Nesoprosopis) excelsa ALFKen, 1931[!] - WARNCKe 1972: 763; 1992: 768.

Material examined: Bingöl: Genç, 1000 m, 12.08.1985, ${ }^{\star}$, leg. R. Hansen. Malatya: Darende, 1000 m, 16.07.1986, ơ, leg. A.W. Ebmer. Van: 1800 m, 13.07.1987, ㅇ, leg. R. Hansen.

Remarks: H. excelsus has a small distribution network. From Turkey, three provinces are added to the distribution records. It is a montane species, which occurs at altitudes above $1000 \mathrm{~m}$. The species is currently recorded from regions of Eastern and South-Eastern Anatolia. It is a sparsely collected species in Turkey (5 provinces).

Distribution: Armenia, Jordan, Iran, Syria; Turkey: Şanlıurfa, Hakkâri (WARNCKE 1972; 1981); Bingöl, Malatya, Van.

\section{Hylaeus (Prosopis) gibbus SAUNDERS, 1850}

Hylaeus (Prosopis) gibbus SAunders, 1850: 59, ․ Greece: Epirus.

Prosopis (Nesoprosopis) gibba gibba (SAUnders, 1850) WARNCKE 1972: 759-760; 1992: 748-750.

Hylaeus (Prosopis) gibbus SAUnders, 1850 - Aliev 1986: 261. Straka \& Bogusch 2011: 61-63; Dathe \& ProshChaly KiN 2018: 71 .

Material examined: Antalya: Arapsuyu, 5 m, 30.06.2002, $2 \sigma^{\star} \sigma^{\star}, 3$ ㅇ 우, 15.10.2003, $2 \sigma^{\star} \sigma^{\star}, 3$ ㅇ ․, leg. H. Özbek (on Mentha longifolia); Alanya, Konakl1, 36 $58 \mathrm{~N} 31^{\circ} 89 \mathrm{E}$, 01.08.2009, $6 \sigma^{\star} \sigma^{\star}, 4$ ㅇ ㅇ , leg. C. Schmid-Egger. Ardahan: Çamlıçatak, 2200 m, 10.08.1976, ㅇ, leg. H. Özbek (on Eryngium sp.). Artvin: Yusufeli, İşhan, 700 m, 29.06.2003, 2 우, leg. H. Özbek (on Sinapis arvensis; Altıparmak, 1200-1700 m, ơ, ㅇ, leg. P. Hartmann; Kaçkar Dağı, $1700 \mathrm{~m}, 15.07 .1995,2$ ㅇ ㅇ, leg. Gelbrecht; Sarıgöl, $900 \mathrm{~m}, 16.07 .1995,2 o^{\star} o^{\star}$, leg. Gelbrecht; Erzincan: Avcilar, $1250 \mathrm{~m}, 04.08 .2003,4 o^{\star} \sigma^{\star}$, leg. S. Çoruh (on Daucus carota). Erzurum: Atatürk University research field, 6 nolu kuyu, 1950 m, 30.07.1976, ơ leg. Özbek (on Medicago sativa); İriağaç, 08.08.2000, $2 \sigma^{\star} o^{x}$, leg. H. Özbek; Tortum, Bağbaşı, 1600 m, 11.09.2001, ơ, ㅇ, leg. S. Çoruh; Uzundere, Balıklı, 1040 m, 29.06.2003, 2 우, leg. H. Özbek, S. Çoruh; Denizbaşı, 1000 m, 20.09.2000, 2 ㅇ ㅇ , leg. H. Özbek. Isparta: Egirdir, Yukarı Gökdere, 1000 m, 25.05.2004, 2 o $^{\star} o^{\star}$, leg. H. Özbek (on Melilotus officinalis). İstanbul: ? + , leg. Hed (coll. RM Stockholm). Kars: Sarıkamıș, Karakurt, TCK Çeşmesi, $1501 \mathrm{~m}, 40^{\circ} 08 \mathrm{~N} 42^{\circ} 21 \mathrm{E}, 05.08 .20022$ 우 우, 14.08.2007, $o^{*}, 16.08 .2002,2 o^{*} \sigma^{*}, 2$ 우, 19.08.2003, 2 ㅇ 이, leg. H. Özbek (on Centaurea solstitialis, Daucus carota). Konya: Çumra, 1016, 13.08.2000, ơ, leg. M. Kesdek. Mersin: Mut, Dereköyü, 02.09.1987, ㅇ, leg. H. Özbek. Muğla: Bodrum, 20.07.2002, ơ , leg. E. Kwast. Nevşehir:

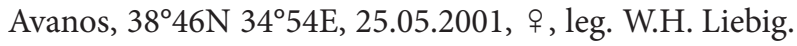
Osmaniye: Kadirli, Karatepe, 500 m, 14.05.2002, 2 우 우, leg. H. Özbek.

Remarks: H. gibbus is widespread throughout the country. Eight more provinces are added here. STRAKA \& 
Bogusch (2011) describe H. gibbus as a thermophilic Mediterranean species that occurs mainly in bushy, grassy, steppe or ruderal biotopes. In the present study, specimens were collected in various biotopes, but mostly in open landscapes, from $5 \mathrm{~m}$ to $2200 \mathrm{~m}$ altitude. Flowers visited: Centaurea solstitialis, Eryngium sp., Medicago sativa, Melilotus officinalis, Mentha longifolia, Sinapis arvensis. The flight season extends from the beginning of May to mid-October with a peak in July. It is frequently recorded in Turkey (21 provinces).

Distribution: Palaearctic. In Turkey: Adana, Aydın, Bilecik, Bursa, Denizli, Hatay, İstanbul, Konya, Kütahya, Muğla, Mersin, Nevşehir, Şanlıurfa (WARnCKE 1972); Antalya, Ardahan, Artvin, Erzincan, Erzurum, Isparta, Kars, Osmaniye.

\section{Hylaeus (Prosopis) incongruus FöRSTER, 1871}

Hylaeus incongruus Förster, 1871: 998-999, ơ ㅇ. Austria. Prosopis (Nesoprosopis) gibba confusa (Nylander, 1852) WARNCKe 1972: 759.

Hylaeus (Prosopis) incongruus Förster, 1871 - STRAKA \& Bogusch 2011:63-65; Dathe \& Proshchalykin 2018: 71-72.

Material examined: Erzurum: Olur, Köprübaşı road side, $1000 \mathrm{~m}, 25.06 .2001 \mathrm{o}^{\star}$, ㅇ; Yeşilbağlar Central, $1200 \mathrm{~m}$, 25.06.2001, †, leg. H. Özbek (on Taraxacum sp.); 25.06.2001, क, leg. J.G. Rozen (coll. AMNH), Oltu, Çamlıbel, 1600 m, 26.07.2000, ơ, ㅇ, leg. H. Özbek.

Remarks: STRAKA \& Bogusch (2011) characterized $H$. incongruus as a thermotolerant species that normally occurs in sandy biotopes, both in dry and wetlands. The present study also confirms their claims, our specimens were taken from wetlands, meadows and pastures in a narrow valley basin between $1000-1600 \mathrm{~m}$ altitude. Interestingly, in Turkey this species seems to be confined to this valley located in the north-eastern part of Erzurum Province (Figs 16.8, 17.9). It is rarely recorded (1 province).

Distribution: Throughout Europe to Eastern Siberia (Straka \& Bogusch 2011; Proshchalykin \& Dathe 2012: 27). Turkey: Erzurum. New to Turkey.

\section{Hylaeus (Prosopis) maculatus (AlFken, 1904)}

Prosopis variegata var. maculata Alfken, 1904: 323, 운. Algeria: Oran.

Prosopis (Nesoprosopis) variegata maculata AlfKen, 1904 WARNCKE 1972: 762; 1981: 186; 1985: 59; 1992: 752.

Hylaeus (Prosopis) maculatus (ALFKen, 1904) - ITIS (2020).
Material examined: Gümüşhane: Torul, $1000 \mathrm{~m}$, 12.07.1985, 40 $34 \mathrm{~N} 39^{\circ} 17 \mathrm{E}, 30^{*} o^{*}$, leg. M. Schwarz (coll. Schwarz/Ansfelden). Siirt: $10 \mathrm{~km} \mathrm{S,} 37^{\circ} 51 \mathrm{~N}$ $41^{\circ} 57 \mathrm{E}, 23.06 .1985$, ㅇ, leg. M. Schwarz (coll. Schwarz/ Ansfelden).

Remarks: H. maculatus occurs in eastern Turkey in two provinces. The Discover Life database (Ascher \& PiCKering 2019) also lists the species for Turkey without giving any further details. It is rarely found in Turkey ( 2 provinces, Fig. 17.VIII).

Distribution: Jordan, Iran, Israel, Lebanon, Syria, Turkey, Egypt, Algeria (Ascher \& Pickering 2019); Turkey: Gümüşhane, Siirt.

\section{Hylaeus (Prosopis) meridionalis FöRSTER, 1871}

Hylaeus meridionalis Förster, 1871: 890, 우‥ Galicia, Ukraine.

Prosopis effasciata Alfken, 1931: 178, ㅇ. . Asia Minor: Tarsus.

Prosopis (Nesoprosopis) variegata meridionalis (FörsteR, 1871) - WARNCKE 1972: 762; 1981: 186; 1992: 751.

Hylaeus (Prosopis) meridionalis Förster, 1871 - Aliev 1986: 263. Dathe \& Proshchalykin 2018: 72.

Material examined: Adana: Pozant1, 06.07.1983, ơ , leg.

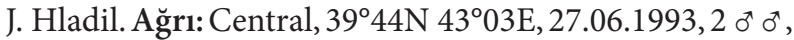

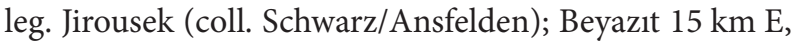
1600 m, 02.07.1985, ㅇ, leg. CJ. Zwakhals. Aksaray: Yeşilova 15 km E, 900 m, 03.7.1984, , leg. A.W. Ebmer. Amasya: 425 m, 27.06.1996, ơ, ㅇ, leg. Kadlec/Vorisek. Ankara: Şereflikoçhisar, Hotalı 850 m, 14.07.1998, $20^{\star} o^{\star}$, leg. C. Güçlü. Antalya: Arıkanda, $36^{\circ} 28 \mathrm{~N} 30^{\circ} 07 \mathrm{E}$, 11.07.1998, o $0^{*}+$, leg. C. Schmid-Egger (coll. E-G); $6 \mathrm{~km}$ SE İbradi, $1260 \mathrm{~m}, 37^{\circ} 12 \mathrm{~N} 31^{\circ} 55 \mathrm{E}, 31.07 .2009$, 2 우 우, leg. C. Schmid-Egger (shrubland); Alanya, 50-100, 28-30.07.1985, 2 우 우, leg. R. Hensen; Demirtaş, $100 \mathrm{~m}$, 29.07.1985, 우, leg. R. Hensen; Konakl $10 \mathrm{~km} \mathrm{W,} \mathrm{36} 58 \mathrm{~N}$ $31^{\circ} 89 \mathrm{E}, 01,08.2009,20^{\star} \sigma^{\star}, 7$ ㅇ 우 , leg. C. Schmid-Egger (coll. E-G); Gündoğmuş, Pembelik, $1090 \mathrm{~m}, 36^{\circ} 83 \mathrm{~N}$ $32^{\circ} 05 \mathrm{E}, \quad 02.08 .2009,11 \mathrm{o}^{\star} \mathrm{o}^{\star}, 3$ 우 우 leg. C. SchmidEgger; Akseki, 1200 m, 09.08.1985, ㅇ, leg. P. van Ooijen; Artvin: Yusufeli, İşhan, 700 m, 15.06.2006, 2 우 이 leg. H. Özbek (Sinapis arvensis; Yusufeli, Demirkent, Salekör, 1600 m, 01.09.1995, †, leg. P. Hartmann. Bingöl: Genç, 1000 m, 12.08.1985, ơ , †, leg. R. Hensen; Genç $15 \mathrm{~km}, \mathrm{~S}, 1400 \mathrm{~m}, 13.08 .1985,2$ 우, leg. R. Hensen; Solhan, $2122 \mathrm{~m}, 24.06 .2000,2 \sigma^{\star} o^{\star}$, leg. M. Kesdek. Bitlis: Ahlat near Van Lake, 14.07.1996, ơ, leg. Tyrner/ Vorisek; Ahlat, $1750 \mathrm{~m}, 15.08 .1985$, ơ, leg. R. Hensen; Tatvan, 16.08.1985, $20^{\star} o^{\star}$, + , leg. R. Hensen (coll. Schwarz/Ansfelden). Bolu: Çerkeş, Kurtçimeni, 700 m, 12.07.1995, ơ , leg. Gelbrecht/Schwa. Burdur: Çeltikçi, 02.09.1983, $2 \sigma^{\star} o^{*}$, leg. J. Timmer. Bursa: $15 \mathrm{~km} \mathrm{~W}$ of Armutlu, 50 m, 27.07.1987, ơ, leg. P. v. Ooijen. Çankırı: Ilgaz, Ilgaz dağları, 900 m, 13.07.1995, ㅇ, leg. Gelbrecht/ 
Schwa. Denizli: Pamukkale, 1000 m, 26.07.1985, 우, leg. P. v. Ooijen; Tavas, Kazıkbeli Geçidi, 1180, 03.07.1990, $\sigma^{\star}$, + , leg. AW. Ebmer. Edirne: Keşan, Mecidiye, 50 m, 29.07.2003, $2 \sigma^{\star} \sigma^{\star}$, leg. Ö. Çalmaşur. Erzincan: Refahiye, $1700 \mathrm{~m}, 15.07 .1984$, ơ , leg. AW. Ebmer; Tercan, Topdağ $1, \quad 1655 \mathrm{~m}, \quad 04.08 .2003,4 \sigma^{\star} \sigma^{*}$, leg. S. Çoruh. Erzurum: Atatürk University, 1900 m, 03.07.2007, $2 \sigma^{\star} o^{\star}$, leg. J. Ascher/ G. Rozen (coll. AMNH); Research field, 06.06.1965, ơ, leg. H. Özbek (on Onobrychis viciifolia); 01.09.1971, female, leg. M. Doğanlar (on Eryngium campestre); İspir, 2800 m, 13.07.1974, ơ , leg. K. Borkowski; Kan, 20.08.1970, 우, leg. H. Özbek (on Daucus carota); Akören, $39^{\circ} 47 \mathrm{~N} 41^{\circ} 09 \mathrm{E}, 22.07 .2003$, ㅇ, leg. J.G. Rozen, H. Özbek (coll. AMNH); Çat yolu, Devlet Su İşleri, Gölet, 3947N $41^{\circ}$ 09E, 09.07.2004, $5 \sigma^{\star} o^{x}$, leg. J.G. Rozen, H. Özbek

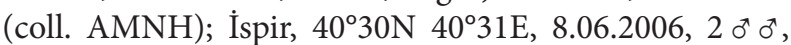
leg. J.G. Rozen, H. Özbek (coll. AMNH); Oltu, Çamlıbel, $1600 \mathrm{~m}, 26.07 .2000,2 \mathrm{o}^{\star} \mathrm{o}^{\star}$, leg. H. Özbek; Çamlıbel $22 \mathrm{~km}$, WSW, 1600 m, 07.07.2001, ơ, leg. J. Ascher, H. Özbek, J.G. Rozen (coll. AMNH); Sarisaz, 1450 m, 21.06.2000, $2 \sigma^{\star} o^{\star}$, leg. Ö. Çalmaşur; Tutmaç-Başaklı border, $1900 \mathrm{~m}$, 01.07.2000, $3 o^{\star} o^{\star}$, Karakol, 1700 m, 01.07.2000, ơ, leg. Ö. Çalmaşur (E. campestre); Tortum, 1700 m, 16.07.1987, ${ }^{\star}$, leg. R. Hensen. Eskişehir: Sakari, Ilıca, 06.07.1987, ơ, leg. Prudek/Ríha. Hakkâri: N 2000 m, 21.07.1986, $6 o^{\star} o^{\star}, 2$ ㅇ 우, leg. AW. Ebmer. Varegöz, $37^{\circ} 25 \mathrm{E} 44^{\circ} 13 \mathrm{~N}$, 1650-1750 m, 02-6.08.1986, 2 o $^{\star} \sigma^{\star}, 2$ ㅇ leg. S.M. Blank; Sat Dağ $1,37^{\circ} 25 \mathrm{E} 44^{\circ} 13 \mathrm{~N}, 1700 \mathrm{~m}, 04.08 .1983$, ơ , leg. W. Schacht; Yüksekova, $20 \mathrm{~km} \mathrm{E,} 2200 \mathrm{~m}, 23.07 .1986$, $2 o^{\star} o^{\star}$, ㅇ, leg. A.W. Ebmer; $30 \mathrm{~km} \mathrm{W,} \mathrm{1850,} \mathrm{20.07.1986,}$ $0^{\star}$, leg. AW. Ebmer. Isparta: Eğirdir, Novada Ulusal Park, Yazılı Kanyon, 08.08.1999, क, leg. I. Brunk. İstanbul: 130 m, Şile, 29.07.1983, †, leg. P. van Ooijen. İzmir: Bergama, 300 m, 22.07.1985, ㅇ, leg. R. Hensen; Ödemiş, Bozdağ, $1000 \mathrm{~m}, 38^{\circ} 22 \mathrm{~N} 27^{\circ} 58 \mathrm{E}, 14.06 .1985$, ㅇ, leg. H. Rausch. Kahramanmaraş: Göksun, Salyan-Ahmetçik arası, 10.07.1990, ㅇ, leg. A.W. Ebmer. Karaman: 10 km S,

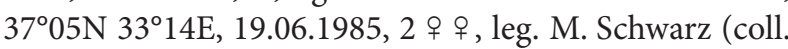
Schwarz/Ansfelden). Kars: Sarıkamış, Karakurt, $40^{\circ} 08 \mathrm{~N}$ $42^{\circ} 21 \mathrm{E}, 1500$ m, 19.06.2007, 2 ㅇ , leg. H. Özbek. Kayseri: Develi, Gezbeli Geçidi, 1750-1900 m, 06.07.1984, o, leg. A.W. Ebmer. Konya: $25 \mathrm{~km} \mathrm{~N}, 11.06 .1966,{ }^{*}$, + , leg. H.H.F. Hamman; Akşehir, Sultandağları, 07.07.1990, $o^{*}$, leg. A.W. Ebmer; Beyșehirr, 13-19.06.1966, $5 o^{\star} o^{*}$, H.H.F. Hamman: Bozkır, Sarığlan, 1000 m, 25.07.2003, $2 \sigma^{\star} o^{\star}$, leg. M. Kesdek; Güneysınır, Habiller, 1100 m, 04.08.2002, 2 ㅇ 우, leg. M. Kesdek. Malatya: Doğanşehir, Reşadiye geçiti, 1500 m, 12.07.1990, ㅇ, leg. A.W. Ebmer. Mersin: Silifke, Uzuncaburç, 400 m, 02.08.1985, ㅇ, leg. R. Hensen; , Kargican, 200 m, 04.08.1985, 2 o $^{\star} o^{\star}, 6$ ㅇ ㅇ, leg. R. Hensen; Mut, 20 km S, 11.07.1996, 우, leg. Brechtel/Ehrmann; Ilıca, 07.06.1966, ㅇ, leg. H.H.F. Hamman. Muğla: Bodrum, 30 m, 25.07.2002, †, leg. E. Kwast. Nevşehir: $2 \mathrm{~km} \mathrm{S,} \mathrm{1250,} \mathrm{04-19.07.1984,} \mathrm{o*} \mathrm{,} \mathrm{leg.}$ A.W. Ebmer; Avanos 10 km S, 1000 m, 22.06.1987, ${ }^{\star}$, leg. R. Hensen; Göreme, 1000 m, 09.07.1988, $0^{\star}$, leg. C. Schmid-Egger (coll. AMNH); Kaymakl1, 1200 m, 23.06.1987, o, leg. R. Hensen; Ürgüp, 1100 m,
11.08.1985, $2 \sigma^{\star} o^{\star}$, leg. P. van Ooijen; Topuzdağ 1 Geçidi, 05.07.1984, 2 우 이 leg. A.W. Ebmer. Niğde: Bokar Dağları, Ulukışla, Çiftehan, 1700-1800 m, 17.07.1990, $20^{\top} \sigma^{\star}$, leg. A.W. Ebmer. Sivas: Gürün 15 km E, 1600 m, 07.07.1984, ơ , leg. A.W. Ebmer. Şanlıurfa: Birecik, 400 m, 06.08.1985, ㅇ, leg. R. Hensen. Tunceli: Ovacik 17 W, $1200 \mathrm{~m}, 19.08 .1985 ; 3 \sigma^{\star} \sigma^{\star}, 2$ 우, eg. R. Hensen. Van: Merkez, $38^{\circ} 30 \mathrm{~N} 43^{\circ} 24 \mathrm{E}, 28.06 .1993$, o $0^{\star}$, o , leg. M. Halada (coll. Schwarz/Ansfelden); Gevaş, Göllü, $38^{\circ} 43 \mathrm{~N} 43^{\circ} 19 \mathrm{E}$, 29.06.1993, 우, leg. M. Halada (coll. Schwarz/Ansfelden). Yalova: Termal, 30 m, 28.08.1985, ㅇ, leg. R. Hensen.

Remarks: We can add 25 provinces here to the known range of $H$. meridionalis. The available records show that this species is the most widespread and most abundant in the country. It is active from June to the end of August and occurs from warmer (seashores) (Antalya, Muğla, Yalova) to colder regions (Erzurum, $2800 \mathrm{~m}$ ), mostly in open areas. Known flowers visited: Daucus carota, Eryngium campestre, Onobrychis viciifolia and Sinapis arvensis. It is frequently recorded in Turkey (46 provinces).

Distribution: Palaearctic from Morocco to Kazakhstan. In Turkey: Mersin (loc. typ. as Prosopis effasciata Alfken, 1931); Adana, Amasya, Ankara, Antalya, Artvin, Aydın, Balıkesir, Bursa, Çanakkale, Denizli, Hatay, İstanbul, Kastamonu, Konya, Kütahya, Manisa, Mersin, Nevşehir, Tekirdağ, Tokat (WARncke 1972); Ağrı, Aksaray, Bingöl, Bitlis, Bolu, Burdur, Çankırı, Edirne, Erzincan, Erzurum, Eskişehir, Hakkâri, Isparta, İzmir, Kahramanmaraş, Karaman, Kars, Kayseri, Malatya, Muğla, Niğde, Sivas, Şanlıurfa, Tunceli, Yalova.

\section{Hylaeus (Prosopis) pictus (Smith, 1853)}

Prosopis picta Smith, 1853: 25-26, ㅇ o $0^{7}$. Spain, Portugal.

Prosopis (Nesoprosopis) gibba picta SMITH, 1853 - WARNCKE 1972: 760; 1992: 750.

Hylaeus (Prosopis) pictus (Sмith, 1853) - Aliev 1986: 262.

Material examined: Adana: Çataalan, 04.04.2002, $\sigma^{*}$, leg. P. Bogusch (coll. Petr Bogusch); Yumurtalik, 31.07.1998, o', leg. T. Osten. Antalya: Akseki, $1200 \mathrm{~m}$, 09.08.1985, ㅇ, leg. P. v. Ooijen; Alanya, 50 m, 28.07.1985, $30^{\top} o^{-1}, 3$ 우 우, leg. R. Hensen; İncekum, $20 \mathrm{~km} \mathrm{~W}$ Alanya, 20 m, 07.08.1985, 7 o $^{\star}$, leg. P. v. Ooijen; Kaş, Kalkan, 13.09.1951, 2 o $^{\star}$, leg. H. A. Guenin; Kasaba, 15.09.1951, ơ , leg. H. A. Guenin; Kemer, 08.09.1951, o ${ }^{\star}$, leg. H.A. Guenin; Manavgat, 50 m, 31.07.1985, ơ, 우, leg. R. Hensen; 6 km SW Manavgat, 20 m, 05.08.1985, 4 o $^{\star} o^{\star}$, 2 우 우, leg. P. v. Ooijen; Demirtaş, $100 \mathrm{~m}, 29.07 .1985$, 2 ơ $^{\star}, 2$ ㅇ 우, leg. R. Hensen. Bursa: 300 m, 20.07.1987, ơ; $225 \mathrm{~m}, 24.08 .1985,7$ o $o^{\star}, 2$ 우; Mudanya, $25 \mathrm{~m}$, 25.08.1985, 3 đ $^{\top}$, + , leg. R. Hensen; Armutlu, $30 \mathrm{~m}$, 20-21.08.1985, $17 o^{\star} o^{\star}, 3$ 우 우, leg. P. v. Ooijen; Hatay: Dörtyol, Payas, 10.04.2002, 2 ㅇ ㅇ, leg. P. Bogusch (coll. Petr Bogusch); İskenderun, Belen S, 28.08.1983, $4 o^{\star} \sigma^{\star}$, 
ㅇ, leg. J. Timmer, Samandağ1, $12 \mathrm{~km} \mathrm{E,} \mathrm{05.07.1998,} \mathrm{ơ,}$ leg. T. Osten. İstanbul: Sozyez? 30.08.1950, 2 우 ㅇ, leg. A. Mochia; Karacaköy, 41 $09 \mathrm{~N}$ 2946E, 30.07.1988, 우, leg. Madl (coll. Schwarz/Ansfelden). Mersin: 26.07.1997, 4 우 오 , leg. J. Timmer; Erdemli, 100 m, 05.07.1986, 우, leg. P. van Ooijen; Ovacık, 50-100 m, 14.07.1998, ㅇ, leg. M. Riha; Silifke, $50 \mathrm{~m}, 02.08 .1985,0^{\star} ; 28 \mathrm{~km} \mathrm{NE}$ Korykos, 03.08.1985, 3 o ơ $^{*} 9$ 우 우, Uzuncaburç, $400 \mathrm{~m}$, 02.08.1985, 2 o $^{*}$, ㅇ ; Kargican, 200 m, 04.08.1985, 2 o $^{\star}$ o $^{*}$, leg. R. Hensen. Sivas: Gürün, Şuğul, 1400 m, 13.07.1990, ㅇ, A.W. Ebmer. Şanlıurfa: Halfeti, 400 m, 28.06.1987, ơ, R. Hensen; $37^{\circ} 15 \mathrm{~N} 37^{\circ} 52 \mathrm{E}$, ơ; 27.05.1987, 25.07.1987, ơ, leg. Madl (coll. Schwarz/Ansfelden); 425 m, 07.08.1985, ㅇ, leg. R. Hensen. Yalova: Termal, 300 m, 28.08.1984, $2 o^{\star} o^{x}, 4$ ㅇ ㅇ, leg. R. Hensen.

Remarks: $H$. pictus was previously known from Adana and Hatay only. Now we can add seven provinces to the records. STRAKA \& Bogusch (2011) consider the species as thermophilic Mediterranean. Our results confirm this opinion, as the majority of the specimens come from coastal areas and lowlands, mostly of the Mediterranean. The flight season lasts from the beginning of April to the end of August, with a peak in July. It is collected moderately in Turkey (9 provinces).

Distribution: Southern Europe, Cyprus, Iran, Turkey, North Africa. In Turkey: Adana, Hatay (StrakA \& Bogusch 2011); Antalya, Bursa, İstanbul, Mersin, Sivas, Şanlıurfa, Yalova.

\section{Hylaeus (Prosopis) rubosus (WARnCKE, 1981) Fig. 13}

Prosopis (Nesoprosopis) rubosa WARNCKe, 1981: 159-160, ㅇ. Turkey: Birecik. - WARNCKe 1981: 187 as rugosa [!]; 1992: 768.

Hylaeus (Prosopis) rubosus (WARNCKe, 1981) - comb. nov.

Material examined: Gaziantep: Nizip 7 km E, 07.07.1996, ㅇ, leg. Brechtel/Ehrmann. Şanlıurfa: Birecik, $400 \mathrm{~m}$, 27.06.1987, 2 ð ð , leg. R. Hensen.

Remarks: H. rubosus was described from Şanliurfa by WARNCKE (1981). In the present study, Gaziantep is added to its distribution range, which is concentrated to southeastern Anatolia. The collecting localities suggest that it is a species of lowland, warm and dry habitats, occurring in steppes and open areas. It is rarely recorded from Turkey (2 provinces, Fig. 17.IX).

Due to its size and the striking rust-brown colouring, the species is not to be overlooked. In its characters it appears much closer to $H$. variolaris than to $H$. excelsus.

Distribution: Iran, Turkey: Şanlıurfa (type locality) (WARNCKe 1981); Gaziantep.

\section{Hylaeus (Prosopis) rugicollis Morawitz, 1874}

Hylaeus rugicollis Morawitz, 1874: 177-179, ․ Russia: Dagestan, Derbent.

Prosopis (Nesoprosopis) rugicollis (MORAWITZ, 1874) - WARNCKE 1972: 763; 1981: 186-187; 1985: 59; 1992: 752-753.

Hylaeus (Prosopis) rugicollis Morawitz, 1874 - Aliev 1986: 263. Dathe \& Proshchalykin 2017: 37; 2018: 73-74.

Material examined: Ağrı: $1650 \mathrm{~m}, 39^{\circ} 44 \mathrm{~N} 43^{\circ} 03 \mathrm{E}$, 25.06.1993, $70^{\star} o^{\star}, 2$ 우, leg. M. Halada (coll. Schwarz/Ansfelden); Doğubeyazıt, 20.08.1983, ㅇ, leg. P. v. Ooijen. Bayburt: Kop Mt. 2300 m, 25.07.2001, ㅇ, leg. Ö. Çalmaşur. Bingöl: 1125 m, 20.06.1986, o, leg. Kadlec/Vorisek; Genç 15 km S, 1400 m, 13.08.1995, 우, leg. Hensen. Bitlis: Ahlat, 14.07.1996, ㅇ, leg. Tyrner/ Vorisek; 1750 m, 15.08.1980, 2 ㅇ 우 , leg. Hensen. Denizli: 450 m, Pamukkale, 31.05.1966, o, leg. H.H.F. Hamann. Erzurum: Atatürk University research field, 6 nolu kuy 1900 m, 17.06.1970, ㅇ, leg. H. Özbek (on Onobrychis viciifolia); Atatürk University Campus, $2000 \mathrm{~m}, 39^{\circ} 53 \mathrm{~N}$ $41^{\circ} 14 \mathrm{E}, 03.07 .2007, \sigma^{\star}$, leg. J.G. Rozen, J.S. Ascher, H. Özbek (coll. AMNH); 10.07.2007, , leg. H. Özbek (on O. viciifolia); Hınıs, Köprüköy-Söylemez, 1850 m, 19.07.2001, ơ, leg. S. Çoruh (on Melilotus officinalis); Oltu, Subatık, $4 \mathrm{~km}$ WSW of Oltu, 1300 m, 09.07.2007, , leg. J.S. Ascher, H. Özbek, J.G. Rozen (coll. AMNH). Hakkâri: 1750 m, 09.07.1987, o , leg. R. Hensen; Beytüşşebab, 1400 m, 06.07.1987, ơ, ㅇ, leg. Hensen; Süvarihalil Geçidi, 2500 m, 27.06.1985, ơ, leg. M. Schwarz (coll. Schwarz/Ansfelden); Yüksekova $16 \mathrm{~km} \mathrm{SE,} 1700 \mathrm{~m}$, $37^{\circ} 27 \mathrm{~N} 44^{\circ} 25 \mathrm{E}, 28.06 .1985$, o ${ }^{\star}$, leg. M. Schwarz (coll. Schwarz/Ansfelden); $30 \mathrm{~km} \mathrm{W,} 1850 \mathrm{~m}, 20.07 .1986$, o*, leg. A.W. Ebmer. Isparta: Eğirdir, 930 m, 06.06.1986, $3{0^{\top} o^{\top}}^{*}$, + , leg. Kadlec/Vorisek. Kars: Sarıkamış, Kara-

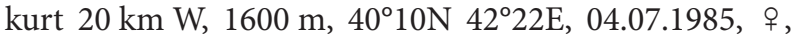
leg. M. Schwarz (coll. Schwarz/Ansfelden). Kayseri: Develi, Kulpak, $38^{\circ} 27 \mathrm{~N} 15^{\circ} 14 \mathrm{E}, 28.05 .2001,3$ o $^{\star}$, leg. W.H. Liebig. Mersin: Mut $11 \mathrm{~km} \mathrm{S,} \mathrm{11.07.1996,} 2$ 우 우, Brechtel/Ehrmann. Muș: Muş Ovası, 1520 m, 18.06.1986, ${ }^{\star}$, ㅇ, leg. Kadlec/Vorisek. Nevşehir: Avanos, Zelve, $1050 \mathrm{~m}, 18.07 .1984$, †, leg. A.W. Ebmer; 22.06.1987, 1000 m, ơ , , leg. R. Hensen; Göreme $10 \mathrm{~km} \mathrm{S,} 1000 \mathrm{~m}$, 22.06.1987, o , leg. R. Hensen; Gülșehir, 20 km N, 1200 m, 20.07.1984, + , leg. A.W. Ebmer; Hacıbektaş, Mucur, 1100 m, 20.07.1984, 2 ㅇ ㅇ, leg. A.W. Ebmer; Ürgüp, Gürüze, 1200-1400 m, 19.06.1986, o, leg. M. Bologna. Şanlıurfa: 550 m, 30.05.1978, ㅇ, leg. M. Schwarz.

Remarks: Eleven provinces are newly added to the distribution area of $H$. rugicollis. The specimens were found in various habitats, mostly in open areas at altitudes of 450-2500 m. In general, the distribution of this species is limited to eastern and central Anatolia. The records show that it is more common in mountain areas. WARNCKE (1981) recorded H. rugicollis at $3800 \mathrm{~m}$ in Iran. The flight time is from May to the end of August. Some of the samples were collected from flowers of Melilotus officinalis and 


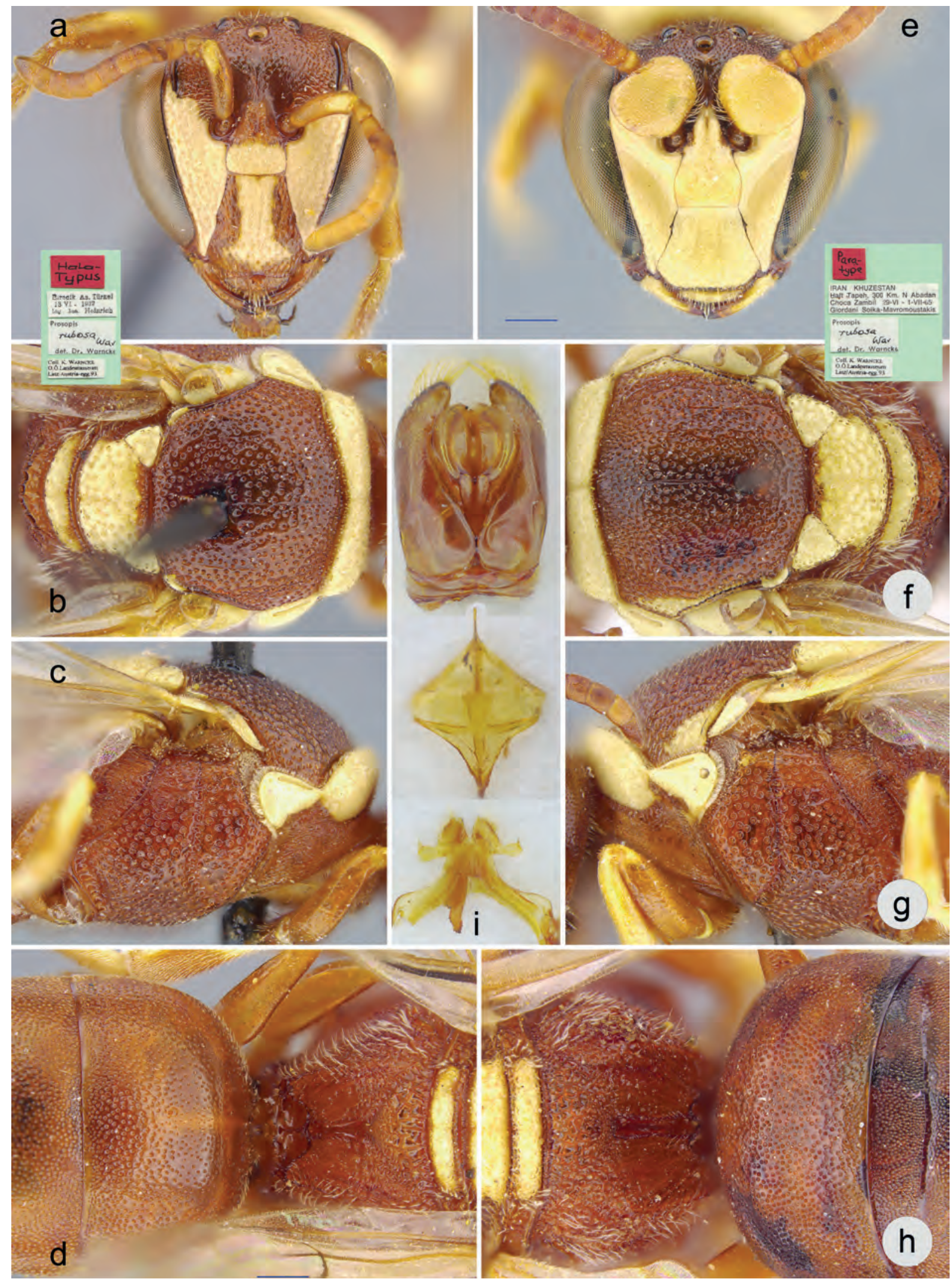

Fig. 13: H. (Prosopis) rubosus (WARNCKe, 1992). Holotype female: a-face, b-mesonotum, c-mesopleuron, d-propodeum and metasoma. Paratype male: e-face, $\mathbf{f}$-mesonotum, $\mathbf{g}$-mesopleuron, $\mathbf{h}$-propodeum and metasoma, $\mathbf{i}$-terminalia. - Scale bar $0.5 \mathrm{~mm}$. 
Onobrychis viciifolia. ÖzBeK (2011) counted H. rugicollis among the pollinating bees of $O$. viciifolia in Erzurum. It is frequently recorded in Turkey (18 provinces).

Distribution: South-eastern Europe, Armenia, Azerbaijan, Iran, Central Asia, Lebanon, Israel, Turkey (DATHE \& Proshchalykin 2018). In Turkey: Amasya, Denizli, Elazı̆̆, Konya, Niğde, Şanlıurfa (WARNCKe 1972); Erzurum (ÖzbeK 1977); Ağrı, Bayburt, Bingöl, Bitlis, Hakkâri, Isparta, Kars, Kayseri, Mersin, Muş, Nevşehir.

\section{Hylaeus (Prosopis) signatus (PANzER, 1798)}

Sphex signata Panzer, 1798: 53, pl. 2, ๆ. Germany.

Prosopis (Nesoprosopis) signata signata (PANZER, 1798) WARNCKE 1972: 760; 1992: 767.

Hylaeus (Prosopis) signatus (PANZer, 1798) - Aliev 1986: 261. Dathe \& Proshchalykin 2018: 74.

Material examined: Ağrı: Doğubeyazıt, Karabulak, $1700 \mathrm{~m}, 39^{\circ} 40 \mathrm{~N} 44^{\circ} 03 \mathrm{E}, 26.06 .1993$, ㅇ , leg. Jirousek (coll. Schwarz/Ansfelden). Aksaray: Sivrihisar Geçidi, 1800 m, $38^{\circ} 16 \mathrm{~N} 34^{\circ} 19 \mathrm{E}, 03.07 .1984$, ơ , leg. A.W. Ebmer. Bitlis: Adilcevaz, 1800 m, 11.07.1984, $7 \sigma^{\star} \sigma^{\star}$, leg. A.W. Ebmer; Ahlat, 1750 m, 15.08.1987, đ, leg. R. Hensen; Van Gölü kıyısı, 14.07.1996, $3 \sigma^{\star} o^{*}$, + , leg. Tyrner/Vorisek. Erzurum: Oltu, Başaklı, $1600 \mathrm{~m}, 11.08 .1977,2$ 우 leg. H. Özbek (Achillea millefolium); Çamlıbel, $1700 \mathrm{~m}$, $22 \mathrm{~km}$ WSW of Oltu, 08.06.2001, o , $;$; $1750 \mathrm{~m}$, 04.07.2004, ơ, + ; 11.07.2004, 2 ㅇ + ; 15.07.2001, ođ, leg. H. Özbek; 08.07.2007, ơ, + , leg. J.S. Ascher, H. Özbek, G. Rozen (coll. AMNH); Pasinler, $5 \mathrm{~km} \mathrm{NE}$ of Pasinler, 1500 m, 01.07.2007, o`, + , leg. J.S. Ascher, H. Özbek, J.G. Rozen (coll. AMNH). Gümüşhane: Torul, 40³4N $39^{\circ} 17 \mathrm{E}, 1000 \mathrm{~m}, 12.07 .1985$, ơ , leg. M. Schwarz (coll. Schwarz/Ansfelden). Hakkâri: Beytüşşebab, $1400 \mathrm{~m}$, 06.07.1997, 3 o $^{\star}, 3$ 우, leg. R. Hensen; Oramar $10 \mathrm{~km} \mathrm{NE}, 1700 \mathrm{~m}, 29.06 .1985,2 o^{\top} o^{\star}$, leg. M. Schwarz (coll. Schwarz/Ansfelden). Isparta: Eğirdir, 15.05.1988, $\sigma^{\star}$, leg. N. Mohr. Kahramanmaraş: Göksun, 1400 m, 26.06.1987, $4 o^{\star} o^{\star}, 2 o^{\star} o^{\star}$, leg. R. Hensen. Kars: Sarıkamış, Akkurt, 1400 m, 18.07.2002, + , Karakurt, TCK Çeşmesi, $1500 \mathrm{~m}, 40^{\circ} 08 \mathrm{~N} 42^{\circ} 21 \mathrm{E}, 08.06 .2005,2$ 우 우 , leg. H. Özbek. Kayseri: Pınarbaşı, 1500 m, 25.06.1987, 3 ㅇ, leg. R. Hensen. Konya: Alaaddin Hill, 1050 m, 20.06.1987, $3 o^{\top} o^{*}$, leg. R. Hensen; Beyşehir, $1150 \mathrm{~m}, 18.06 .1987$, $15 \sigma^{\star} \sigma^{*}, 5$ ㅇ, leg. R. Hensen. Nevşehir: Göreme, $38^{\circ} 40 \mathrm{~N}$ $34^{\circ} 50 \mathrm{E}, 23.06 .1993$, o* , leg. M. Halada (coll. Schwarz/ Ansfelden). Niğde: Çamardı N, Aladağlar, $1750 \mathrm{~m}$, 16.07.1990, 2 ㅇ 우 , leg. A.W. Ebmer. Sakarya: Taraklı, $400 \mathrm{~m}, 40^{\circ} 26 \mathrm{~N} 30^{\circ} 28 \mathrm{E}, 20.05 .1985$, o*, leg. H. Rausch. Sivas: Gürün, Mazıkıran Geçidi, 1650 m, 13.07.1990, $0^{\star}$, ㅇ, leg. A.W. Ebmer. Van: $1800 \mathrm{~m}, 13.07 .1987,3 \sigma^{\star} \sigma^{\star}$ leg. R. Hensen; Başkale, $2200 \mathrm{~m}, 10.07 .1987,2 o^{\star} o^{\star}$, leg. R. Hensen. Gürpınar, Güzelsu, Hoşap, 2200 m, 12.07.1984, 2 우 우, leg. A.W. Ebmer.
Remarks: Fourteen provinces are added here to the distribution records of $H$. signatus. In general, it was collected in areas with continental climate at altitudes from $1000 \mathrm{~m}$ to $2200 \mathrm{~m}$ (Van). Accordingly, it was not collected from the Aegean and Mediterranean coastal areas. It is a common species, both in warmer and cooler regions. The available data show that it mainly prefers steppes and open areas. It is active from the end of May to the end of August. The species is often recorded from Turkey (21 provinces).

Distribution: North Africa and almost all of Europe; Asia Minor, Azerbaijan, Central Asia, introduced to Madeira (Dathe \& Proshchaly kin 2018). In Turkey: Afyonkarahisar, Bursa, İstanbul, Kirıkkale, Konya, Mersin (WARNCKe 1972); Erzurum (ÖzBeK 1977), Ağrı, Aksaray, Bitlis, Gümüşhane, Hakkâri, Isparta, Kahramanmaraş, Kars, Kayseri, Nevşehir, Niğde, Sakarya, Sivas, Van.

\section{Hylaeus (Prosopis) stellatus (WARncke, 1992) Fig. 14}

Prosopis (Nesoprosopis) duckei stellata WARNCKE, 1992: 772, 우. Turkey: Hakkâri, Varegös.

Hylaeus (Prosopis) stellatus (WARncke, 1992) - Dathe \& Proshchalykin 2018: 74.

Material examined: Antalya: $72 \mathrm{~km} \mathrm{SW}$, Finike, Arif

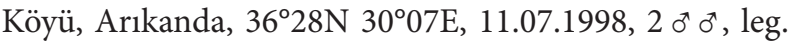
C. Schmid-Egger.

Remarks: WARNCKE (1992) described H. stellatus as a subspecies of $H$.duckei, Prosopis duckei stellata. He correctly recognized that this taxon is different to $H$. duckei s. str., especially in structures of the integument. A number of habitual, colour and sculpture features could be added here, including marked differences in the male terminalia. For the subspecies status, however, no argument is evident. In this study, the species has only been newly found in Antalya, but it is recorded for the first time since the description. In addition, its occurrence in the Mediterranean region is new. Dathe \& Proshchalykin (2018) stated: “This species is by no means isolated and restricted to the southeast of Turkey, as WARNCKE (1972) suspects. It is known from Israel and Kazakhstan." It has a sporadic distribution in Turkey. This species lives in both cooler (Hakkâri) and warmer (Antalya) regions. It has been collected in Turkey only sparsely (4 provinces).

Distribution: Israel, Kazakhstan, Turkey (DATHE \& Proshchalykin 2018). In Turkey: Hakkâri, Siirt and Şırnak (WARNCKE 1992); Antalya. 


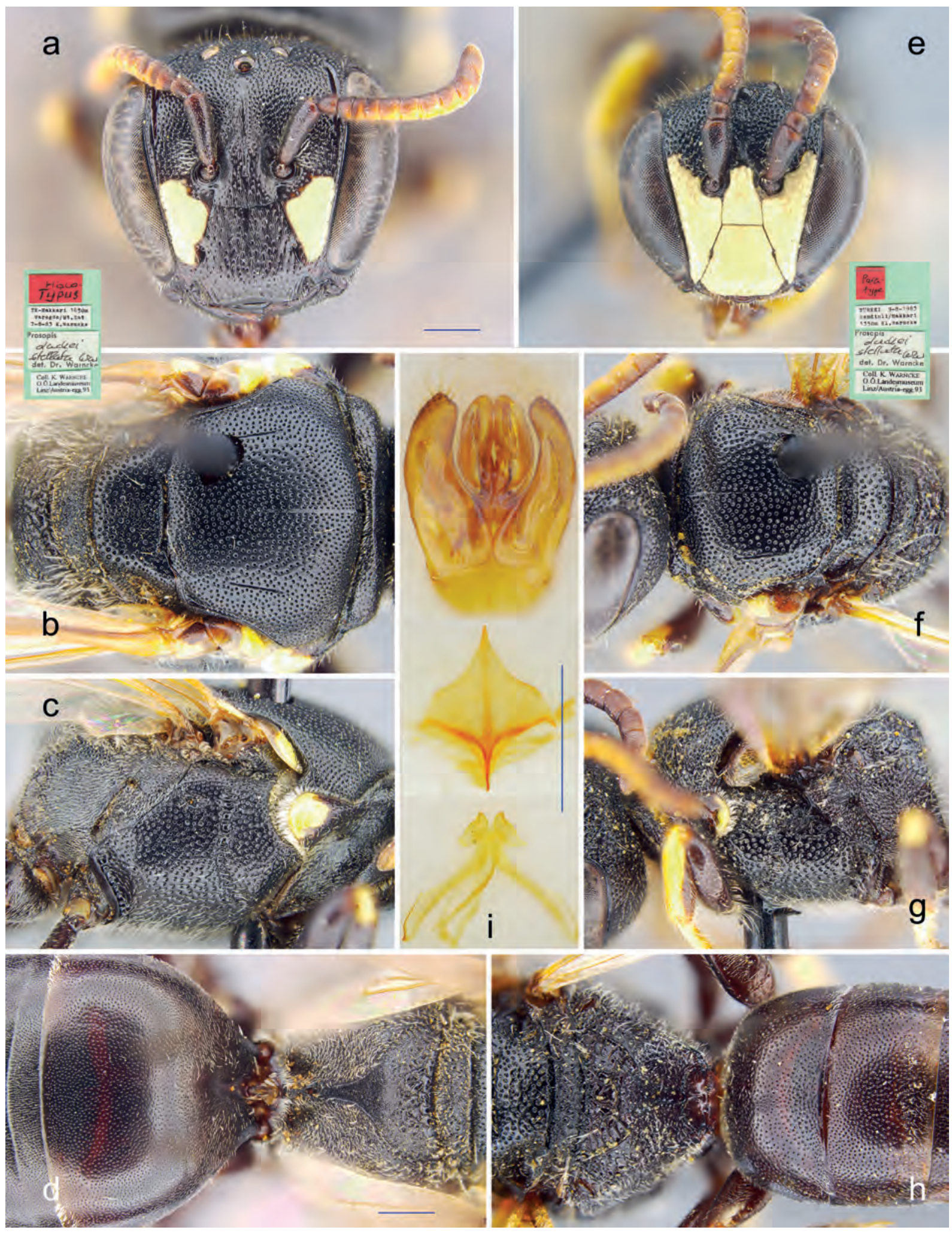

Fig. 14: H. (Prosopis) stellatus (WARnCKE, 1992). Holotype female: a-face, b-mesonotum, c-mesopleuron, d-propodeum and metasoma. Paratype male: e-face, f-mesonotum, $\mathbf{g}$-mesopleuron, $\mathbf{h}$-propodeum and metasoma, $\mathbf{i}$-terminalia. - Scale bar $0.5 \mathrm{~mm}$. 


\section{Hylaeus (Prosopis) trinotatus (PÉREZ, 1896)}

Prosopis trinotata PÉRez, 1896: 64, 욱. Italy: Sicily.

Prosopis (Nesoprosopis) trinotata PÉREZ, 1895[!] - WARNCKe 1972: 761 .

Material examined: Aksaray: Esmekaya, $950 \mathrm{~m}, 38^{\circ} 16 \mathrm{~N}$ $33^{\circ} 22 \mathrm{E}, 16.07 .1998$, ㅇ, leg. C. Schmid-Egger. Antalya: Arapsuyu, Azmak, 5 m, 30.06.2002, o; 04.07.2002, +, leg. H. Özbek (on Mentha longifolia). Balıkesir: Bandırma, Kuşcenneti, 15 m, 07.09.1983, 7 o $^{\star}$, 우 , leg. P. van Ooijen.

Remarks: Three provinces are added to the recorded distribution of H. trinotatus. This species is sporadically distributed throughout the country. The known localities indicate that $H$. trinotatus prefers flat, warm and dry habitats, which occur mainly in steppes and open areas. It is only sparsely collected in Turkey (3 provinces, Fig. 16.9).

Distribution: Southwest Europe, Iraq, Israel, Turkey: Aksaray, Antalya and Balıkesir. New to Turkey.

\section{Hylaeus (Prosopis) variegatus (FABRICIUs, 1798)}

Mellinus variegatus Fabricius, 1798: 265, ㅇ. Europe.

Prosopis (Nesoprosopis) variegata variegata (FABRICIUs, 1798) WARNCKE 1992: 761; 1992: 751-752.

Hylaeus (Prosopis) variegatus (FABricius, 1798) - Aliev 1986: 262. Dathe \& Proshchalykin 2018: 75.

Material examined: Adana: Ceyhan, Kurtkulağ 1 , 29.07.1998, ơ , leg. T. Osten; Yumurtalık, 31.07.1998, ơ, ㅇ, leg. T. Osten. Adıyaman: Gölbaşı, 37²47N 37³9E, 21.06.1985, o*, leg. M. Schwarz (coll. Schwarz/ Ansfelden); Karadut, Nemrut Dağ $1,37^{\circ} 56 \mathrm{~N} 38^{\circ} 47 \mathrm{E}$, 02.07.1993, ㅇ, leg. Halada (coll. Schwarz/Ansfelden).

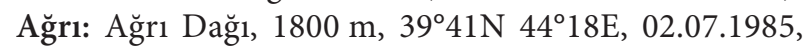
ㅇ, leg. M. Schwarz (coll. Schwarz/Ansfelden); $1650 \mathrm{~m}$, $39^{\circ} 44 \mathrm{~N} 43^{\circ} 03 \mathrm{E}, 27.06 .1993,40^{\top} 0^{\top}, 2$ 우 우, leg. Jirousek; $50 \mathrm{~km} \mathrm{E}, 1800 \mathrm{~m}, 39^{\circ} 43 \mathrm{~N} 43^{\circ} 39 \mathrm{E}, 12.07 .1985,2 \mathrm{o}^{\star} \mathrm{o}^{\star}$, leg. M. Schwarz (coll. Schwarz/Ansfelden); Cumaçay, 1930 m, 23.07.1996, ơ, 우, leg. P. Rasmont (on Cirsium arvense vestitum); Eleşkirt $30 \mathrm{~km} \mathrm{W,2200} \mathrm{m,} \mathrm{14.07.1987,}$ $3 \sigma^{\star} o^{\star}$, ㅇ, leg. R. Hensen. Aksaray: $27 \mathrm{~km}$ SE of Aksaray,

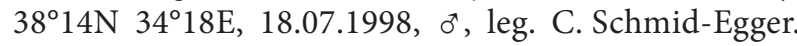
Antalya: Akdeniz University Campus, $15 \mathrm{~m}, 36^{\circ} 53 \mathrm{~N}$ 30³9E, 29.05.2009, 2 우 우, leg. J.S. Ascher, H. Özbek, J.G. Rozen; Alanya, Demirtaş, 100 m, 29.07.1985, 2 o $^{\star} 0^{\star}$, leg. R. Hensen; İncekum 20 km W, 20 m, 07.08.1985, o, leg. P. van Ooijen; Anamur, 3602N 32079E, 21.07.1998, $0^{\star}$, leg. C. Schmid-Egger (coll. AMNH); Çiğlik, 217 m, 02.06.2009, ơ, leg. J.S. Ascher, H. Özbek(coll. AMNH); Kaş, Kasaba, 15.09.1951, ơ, leg. H.A. Guenin; Kemer, 08-09.09.1951, o*, 2 ㅇ 우, leg. H.A. Guenin; Konyaalt1, $3 \mathrm{~m}, \quad 29.05 .2009, \quad o^{\star}$, leg. J.S. Ascher, H. Özbek, J.G. Rozen (coll. AMNH); Konyaaltı Plajı, 3651N $30^{\circ} 38 \mathrm{E}, 2 \mathrm{~m}, 29.05 .2009,2 \sigma^{\star} o^{*}$, + leg. J.S. Ascher, H. Özbek, J.G. Rozen (coll. AMNH; Arapsuyu, Azmak, $10 \mathrm{~m}, 30.06 .2002$, o $o^{\star}, 24.09 .2004,7 o^{\star} o^{\star}$ leg. H. Özbek (on Mentha longifolia); Düzçam, 200 m, 30.05.2004, ơ, leg. H. Özbek (on Vitex agnus-castus); Kumluca, Arrkanda, $36^{\circ} 28 \mathrm{~N} \quad 30^{\circ} 07 \mathrm{E}, 11.07 .1998$, 이 leg. C. Schmid-Egger; Olimpus, 14.07.1996, 2 ○ $^{\star}$, leg. Brechtel/Ehrmann. Artvin: Demirkent, Salekör, 1600 m, 02.09.1995, ㅇ P. Hartmann; Kaçkar, Yaylalar, 2200 m, 17.07.1995, 3 o o $^{\star}$, leg. Gelbrecht/Schwa (on Anchusa sp.). Aydın: Burhaniye, 10 m, 18.07.1985, ơ, leg. P. van Ooijen; Çine, 20-23.09.1951, o*, leg. H.A. Guenin; Söke, 14.08.1985, †, leg. F. Wagner. Balıkesir: Edremit, Ören, $27^{\circ} 02 \mathrm{E} 39^{\circ} 33 \mathrm{~N}, 04.08 .1987$, ㅇ, leg. Dollfuss. Bingöl: Kuruca Geçidi, $1800 \mathrm{~m}$, 08.07.1984, o*, leg. A.W. Ebmer; Solhan, 2120 m, 25.06.2000, ơ, ㅇ, leg. M. Kesdek; 1750 m, 15.08.1985, 2 우 ㅇ, leg. R. Hensen; Tatvan, 1750 m, ㅇ, leg. R. Hensen. Bitlis: Ahlat, 14.07.1996, , leg. Tyrner/Vorisek; Tatvan, 15.08.1985, 2 ㅇ ㅇ, leg. R. Hensen. Bursa: Armutlu, $50 \mathrm{~m}, 27.07 .1986,3 \mathrm{o}^{\star} o^{\star}$, leg. P. van Ooijen; Çağlayan, 10.07.1986, ơ, leg. Prudek/Riha; Uludağ, 225, 24.08.1985, $2 \sigma^{\star} \sigma^{\star}$, leg. R. Hensen. Denizli: Pamukkale, $37^{\circ} 56 \mathrm{~N} 29^{\circ} 08 \mathrm{E}, 14.07 .1998,2$ o $^{\top}$ o $^{\top}$ leg. C. Schmid-Egger. Diyarbakır: Malabadi, $650 \mathrm{~m}, \quad 38^{\circ} 08 \mathrm{~N} \quad 41^{\circ} 12 \mathrm{E}$, 28.05.1985, ơ , leg. H. Rausch. Edirne: Keşan, Mecidiye, $50 \mathrm{~m}, 29.07 .2003$, ơ , leg. Ö. Çalmaşur. Erzincan: $55 \mathrm{~km} \mathrm{~W}, 1600 \mathrm{~m}, 39^{\circ} 44 \mathrm{~N} 38^{\circ} 52 \mathrm{E}, 13.07 .1985,2$ o $^{\top} \mathrm{o}^{\star}$, leg. M. Schwarz (coll. Schwarz/Ansfelden); Çağlayan, $39^{\circ} 36 \mathrm{~N} 39^{\circ} 42$ E, 23.06.2002, ơ ${ }^{*}$, leg. P. Kment (coll. Petr Bogusch); Refahiye, $1700 \mathrm{~m}, 15.07 .1984,0^{*}$, ㅇ, leg. A.W. Ebmer. Erzurum: Atatürk University research field, 12.07.1970, ơ ${ }^{\star}$,, leg. H. Özbek (on Onobrychis viciifolia); 13.07.2004, o'; 18.07.1970, ơ; 29.07.1970, ơ; 17.08.1970, ơ, leg. H. Özbek (on Melilotus officinalis); Atatürk University Campus, $2000 \mathrm{~m}, 39^{\circ} 53 \mathrm{~N} 41^{\circ} 14 \mathrm{E}$, 13.07.2003, 4 우, leg. H. Özbek (on O. viciifolia); 20.06.2008, $3 \sigma^{\star} o^{\star}$, leg. J.G. Rozen, H. Özbek (coll. AMNH); 28.06.2001, ơ , ㅇ, leg. J.G. Rozen, H. Özbek

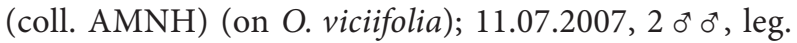
J.S. Ascher, H. Özbek, J.G. Rozen (coll. AMNH); 13-14.07.2001, 4 o o $^{\star}$, 우, leg. H. Özbek (on O. viciifolia) ; 16-21.07.2003, 9 o $^{\star} \sigma^{\star}, 16$ ㅇ , leg. J.G. Rozen, H. Özbek (coll. AMNH); Abdurrahmangazi, 2200 m, 14.07.2000, $\mathrm{O}^{\star}$, leg. C. Güçlü (on Trifolium pratense); Geçit Köy, 2084 m, 27.07.2010, ơ, leg. J.S. Ascher, H. Özbek (coll. AMNH); Hınıs yolu, Mescitli, 1700 m, 19.07.2003, ㅇ, leg. H. Özbek (on Euphorbia sp.); Ilıca, Ağzıcçık Geçidi, 2000 m, 19.07.2003, 3 o $^{\star}$, leg. H. Özbek (on Cephalaria procera); Kargapazarı Mt, 2300 m, 15.07.2002, ơ, leg. H. Özbek on Thymus sp.); Tafta, 20.06.1970, 3 우 우, leg. H. Özbek (on Trifolium repens); Çat yolu, DSİ, Gölet, $39^{\circ} 47 \mathrm{~N} \quad 41^{\circ} 09 \mathrm{E}, 09.07 .2004,0^{\top}, 4$ 우, leg. J.G. Rozen, H. Özbek (coll. AMNH); Köprüköy, İncesu, 2340 m, 03.07.2004, o, leg. S. Çoruh, C. Güçlü (on Astragalus sp.); Oltu, Başaklı, 1700 m, 20.06.1970, 우, leg. H. Özbek (on Echinops sp.); Subat1k, 1300 m, 13.08.2004, ơ , leg. H. Özbek (on Daucus carota); 22 km 
WSW of Oltu, $1700 \mathrm{~m}, 08.07 .2007$, ơ, leg. J.S. Ascher, H. Özbek, J.G. Rozen; Çamlıbel Karakolu, 1900 m, 01.07.2000, ơ, leg. H. Özbek; Palandöken, 27.07.1986, 2200-2400 m, $2 o^{\star} \sigma^{*}$, leg. A.W. Ebmer; Pasinler, Demirdöven baraj1, 1795, 01.07.2007, ơ , leg. J.S. Ascher, H. Özbek, J.G. Rozen (coll. AMNH); $5 \mathrm{~km} \mathrm{NE} \mathrm{of}$ Pasinler, 01-10.07.2007, $4 \mathrm{o}^{\star} \mathrm{o}^{\star}$, leg. J.S. Ascher, H. Özbek (coll. AMNH); Tortum, 1700 m, 16.07.1987, ㅇ, leg. R. Hensen; Tekman, 1953 m, 08.07.2007, ơ, leg. J.S. Ascher, H. Özbek, J.G. Rozen; Uzundere, Aksukapi $11 \mathrm{~km} \mathrm{~N}, 1250 \mathrm{~m}, 29.06 .2003,2$ o $^{\star}$, leg. H. Özbek, S. Çoruh (on Euphorbia sp.). Hakkâri: Beytuşşebab, 1400 m, 06.07.1987, ㅇ, leg. R. Hensen; Varagöz, 1650, $37^{\circ} 25 \mathrm{~N} 44^{\circ} 13 \mathrm{E}, 02.08 .1986$, 우, leg. S.M. Blank; Oramar, $10 \mathrm{~km} \mathrm{NE}, 1700 \mathrm{~m}, 29.06 .1985$, ơ, leg. M. Schwarz (coll. Schwarz/Ansfelden); Yüksekova $10 \mathrm{~km} \mathrm{E,} 2100$ $2200 \mathrm{~m}, 2 \sigma^{\star} \sigma^{\star}$, ㅇ; $30 \mathrm{~km} \mathrm{~W}, 1850 \mathrm{~m}, 19.07 .1986,2$ o $^{\star} o^{\star}$, leg. A.W. Ebmer. Hatay: İskenderun $20 \mathrm{~km} \mathrm{~S}$, 05.07.1996, 5 우, leg. Brechtel/Ehrmann; Belen, 28.08.1983, ơ, leg. J. Timmer; Samandağı, Nur dağları, 06.07.1996, ơ, 2 우, leg. Brechtel/Ehrmann. Iğdır: 19.08.1983, o , leg. J. Timmer; Bayraktutan, 890 m,

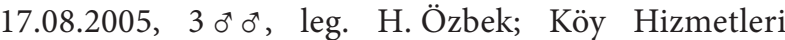
Araştırma İstasyonu, $900 \mathrm{~m}, 31.07 .2002$, đa, leg. M. Kesdek. İstanbul: 30.08.1950, †, leg. A. Mochi; Ömerli, 20.07.1994, , leg. ? (coll. AMNH); Şile, 29.07.1983, o*, leg. P. van Ooijen. İzmir: Bergama, 300 m, 22.07.1985, ơ , leg. R. Hensen. Kars: Sarıkamış, Karakurt $20 \mathrm{~km} \mathrm{W,} 1600 \mathrm{~m}, 40^{\circ} 10 \mathrm{~N} 42^{\circ} 22 \mathrm{E}, 04.07 .1985$, , leg. M. Schwarz (coll. Schwarz/Ansfelden); $30 \mathrm{~km} \mathrm{W,}$ $2100 \mathrm{~m}, \quad 40^{\circ} 20 \mathrm{~N} \quad 42^{\circ} 22 \mathrm{E}, \quad 04.07 .1985,2$ 우, leg. M. Schwarz (coll. Schwarz/Ansfelden). Kayseri: Kermelik, 1320 m, 13.07.1996, ㅇ, leg. D. Flagotier (on Echium italicum). Konya: Beyşehir, 1150 m, 18.06.1987, o , leg. R. Hensen. Malatya: Pütürge, Tepehan, $1340 \mathrm{~m}$, 12.07.1995, 5 ㅇ ㅇ, leg. Y. Barbier (on Echium italicum). Mardin: 1000 m, 02.07.1987, ㅇ, leg. R. Hensen. Mersin: Gülnar, 31.07. 1988, ơ, leg. C. Schmid-Egger; Mut, Dereköy, 02.09.1987, †, leg. H. Özbek; Ortaköy, 11.07.1996, ơ, leg. Brechtel/Ehrmann; Silifke, $50 \mathrm{~m}$, 02.08.1985, $2 \sigma^{\star} \sigma^{*}$, leg. R. Hensen; Karkıcan, 200 m, 04.08.1985, ơ, leg. R. Hensen; Ovacık, Büyükeceli, 50-100 m, 14.07.1998, ơ , leg. M. Riha. Muğla: Bodrum, Salmakis, $37^{\circ} 02 \mathrm{~N} 27^{\circ} 25 \mathrm{E}, 16-21.07 .2001,6 \sigma^{\star} \sigma^{\star}$, 우, leg. F. Burger (on Vitex agnus-castus); Fethiye, 06-07.09.1951, $2 \sigma^{\star} o^{\star}$, ㅇ, leg. H.A. Guenin; Ölüdeniz, 30.07.1985, đ̛, leg. P. van Ooijen; Köyceğiz, 06.05.1998, $0^{7}$, leg. G. Tozlu; Marmaris $56 \mathrm{~km}$ NNW, $15 \mathrm{~km}$ E Milas,

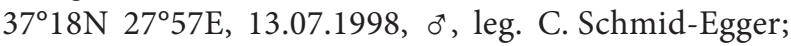
Çamköy, $37^{\circ} 07^{\prime} 70 \mathrm{~N} 27^{\circ} 53^{\prime} 30 \mathrm{E}, 20-22.06 .1998,7$ 우, leg. O. Niehuis. Nevşehir: $2 \mathrm{~km} \mathrm{~S}, 1250 \mathrm{~m}$, 04-19.07.1984, 4 o $^{\star}$, leg. A.W. Ebmer; Avanos, Zelve, $1050 \mathrm{~m}, 14.07 .1990,2$ 우; 17-18.07.1984, ơ , ㅇ, leg. A.W. Ebmer; Göreme, 38³9N 3453E, 17.07.1998, o, leg. C. Schmid-Egger; Kaymaklı, 1200 m, 23.06.1987, $\sigma^{\star}$, leg. R. Hensen; Ürgüp, $1100 \mathrm{~m}, 11.08 .1985,2 \sigma^{\star} \sigma^{\star}$, leg. P. van Ooijen; Topuz Dağ 1 Geçidi W, 1300 m, 05.07.1984, ㅇ, leg. A.W. Ebmer. Niğde: Çiftehan, Bolkar dağları, $\quad 1700-1800 \mathrm{~m}, \quad 17.07 .1990, \quad 2 \sigma^{\star \top} \sigma^{\star}, \quad$ leg. A.W. Ebmer. Osmaniye: Kastabala, 08.07.1996, ơ leg. Brechtel/Ehrmann (on Eryngium sp.). Siirt: $20 \mathrm{~km} \mathrm{~S}$, $500 \mathrm{~m}, 23.06 .1985$, क , leg. C.J. Zwakhals. Sinop: Ayancık, 50 m, 23.07.1977, ㅇ, leg. H. Özbek (on Carduus sp.). Sivas: Gürün $15 \mathrm{~km} \mathrm{W,} 1600 \mathrm{~m}$, 07.07.1984, o, leg. AW. Ebmer; İmranlı $40 \mathrm{~km} \mathrm{E}$, 1700 m, 15.07.1984, ㅇ, leg. A.W. Ebmer; Şuğul vadisi, 1400 m, 31.07.1986, 2 우 ㅇ, leg. A.W. Ebmer; Yeniçubuk, $1100 \mathrm{~m}, 14.07 .1996,2$ 우 ㅇ, leg. P. Rasmont (on Allium cepa; Yıldızeli, Çamlıbel Pass, 1600-1700 m, 16.07.1984, 4 우 오 leg. A.W. Ebmer. Tokat: Pazar, Ballıca Mağarası,

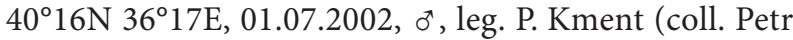
Bogusch). Van: Central, $1800 \mathrm{~m}, 13.07 .1987$, + , leg. R. Hensen; Başkale, 2200 m, 10.07.1987, ơ , ㅇ, 30 km N, 2700 m, 11.07.1987, $2 \sigma^{\star} \sigma^{\star}$, leg. R. Hensen; Güzeldere Geçidi, 2500, 12.07.1984, ㅇ, leg. A.W. Ebmer.

Remarks: In the present study, H. variegatus was collected from 34 provinces throughout the country, including Thrace (European part of Turkey). Our results show that it is widespread and numerous in both warmer and cooler regions, from sea level (Antalya, Muğla) to $2200 \mathrm{~m}$ altitude (Erzurum, Hakkâri) in various habitats. However, it appears concentrated in the provinces of Antalya and Erzurum. The species has a long flight season, from May to September, and probably there is more than one generation. Several plant species were discovered to be visited by $H$. variegatus. Among them are important forage plants in the country: on Onobrychis viciifolia, Trifolium pratense and T. repens (ÖzBEK 2011, 2018). It is frequently collected in Turkey (35 provinces).

Distribution: Transpalaearctic species, from North Africa to Central Asia, Mongolia, China and Russia in the Far East. Frequently recorded from Turkey, Armenia, Azerbaijan, Lebanon and Syria. Turkey: Erzurum (Özbek 1977); Adana, Adıyaman, Ağrı, Aksaray, Antalya, Artvin, Aydın, Balıkesir, Bingöl, Bitlis, Bursa, Denizli, Diyarbakır, Edirne, Erzincan, Hakkâri, Hatay, Iğdır, İstanbul, İzmir, Kars, Kayseri, Konya, Malatya, Mardin, Mersin, Muğla, Niğde, Osmaniye, Siirt, Sinop, Sivas, Tokat, Van.

\section{Hylaeus (Prosopis) variolaris MonawITz, 1876}

Hylaeus variolaris MoRAwITz, 1876: 286-287, o o o . Uzbekistan: Sarafshan.

Prosopis variolaris (MORAWITZ, 1876) - WARNCKE 1981: 154. Hylaeus (Prosopis) variolaris Morawitz, 1876 - DATHE \& Proshchalykin 2017: 43-44; 2018: 76.

Material examined: Erzurum: Atatürk University Campus, 1950 m, 13.07.2004, ơ , leg. H. Özbek (on Melilotus officinalis) (coll. AMNH). Kayseri: Pınarbaşı, $1500 \mathrm{~m}, 25.06 .1987,2 o^{\star} o^{\star}$, leg. R. Hensen. 
Remarks: Exact collecting data of $H$. variolaris were recently published by Dathe \& Proshchalykin (2017, 2018) for Central Asia. Ascher \& Pickering (2019) also report the presence of this species in Turkey and Azerbaijan in "Discover Life", but without precise location information. The species is easy to recognize, so these notifications are quite plausible. In any case, here we report $H$. variolaris as new for the Turkish fauna, and Kayseri is the westernmost distribution point of this species as a whole. $H$. variolaris is only rarely collected in Turkey (2 provinces, Figs 16.10, 17.X).

Distribution: Azerbaijan, Tajikistan, Turkmenistan, Uzbekistan (Dathe \& Proshchalykin 2017; 2018). In Turkey: Erzurum, Kayseri. New for Turkey.

\section{Subgenus Spatulariella PoPOV, 1939}

\section{Hylaeus (Spatulariella) alpinus (MoraWITZ, 1867)} Fig. 1a

Prosopis alpina MoRAwitz, 1867: 50, 우 $0^{\star}$. Switzerland: St. Moritz.

Prosopis (Spatulariella) alpina MoraWITZ, 1867 - WARNCKe 1972: 758

Hylaeus (Spatulariella) alpinus (Morawitz, 1867) - Dathe, SCHEUCHL \& OCKERMÜLLER 2016: 18, 35.

Material examined: Bursa: Uludağ, 1700 m, 19.07.1987, 2 우, leg. A.W. Ebmer.

Remarks: H. alpinus is a European species, which according to DATHE et al. (2016) is found on mountain locations, preferably at altitudes between $1000 \mathrm{~m}$ and $2100 \mathrm{~m}$ (Fig. 17.10).

Distribution: Pyrenees, Alps (up to $1900 \mathrm{~m}$ ), Tatras, Balkans, Northern Greece (Varnous, Pindos). Ascher \& PiCKering (2019) in "Discover Life" state that this species occurs in Turkey, but the dots on their maps refer only to the country, not to a location.

\section{Hylaeus (Spatulariella) adspersus (ALFKEN, 1935)} Fig. 1c

Prosopis adspersa Alfken, 1935: 177, 우. Palestine.

Prosopis (Spatulariella) adspersa ALFKEN, 1935 - WARNCKE 1972: 758; 1981: 164; 1985: 58; 1992: 768.

Material examined: Artvin: Murgul, Damar, 01.07.1997, ㅇ, leg. Prudek/ Ríha. Kayseri: Erciyes Mt, 1850 m, 05.07.1984, $2 \sigma^{\top} \sigma^{\top}$, leg. A.W. Ebmer. Mersin: Mezitli, Akarca, $25 \mathrm{~km}$ WNW, $805 \mathrm{~m}, 14.05 .1959$, ㅇ, leg. V123. Sivas: $40 \mathrm{~km} \mathrm{~W} \mathrm{Gürün,} \mathrm{Ziyaret} \mathrm{Geçidi,} 2000 \mathrm{~m}$, 15.07.1986, ơ , leg. A.W. Ebmer.
Remarks: H. adspersus can be considered to be a southern Mediterranean species that does not occur in Europe. Here, three Turkish provinces are added to the known distribution. The species has a sporadic distribution. Artvin is the most eastern and northern distribution record of this species in general. It is only sparsely collected in Turkey (6 provinces).

Distribution: Tunisia, Iraq, Israel; Turkey: Mardin, Mersin, Sanlıurfa (WARNCKE 1972); Artvin, Kayseri, Sivas.

\section{Hylaeus (Spatulariella) armeniacus (WARNCKE, 1981) Fig. if}

Prosopis (Spatulariella) armeniaca WARNCKE, 1981: 163-165, 우. Turkey: Horasan [Erzurum]. - WARNCKe 1985: 58; 1992: 768.

Hylaeus (Spatulariella) armeniacus (WARNCKe, 1981) - ITIS (2020).

Material examined: Adıyaman: Nemrut Mt National Park, Karadut, 3756N 3847E, 02.07.1993, ơ, leg. Jirousek (coll. Schwarz/Ansfelden). Ağrı: $10 \mathrm{~km}$ South, 1650 m, 26.07.2003, 2 o o o $^{\star}$ leg. H. Özbek; Hamur, 1650 m, 26.07.2003, 2 đ $^{\star} \sigma^{\star}$, leg. H. Özbek. Sanlıurfa: Siverek, Karabahçe, 800 m, 09.05.2002, $2 \sigma^{\star} \sigma^{*}$, leg. H. Özbek. Tunceli: Ovacik $17 \mathrm{~km} \mathrm{W,} 1250 \mathrm{~m}, 19.08 .1985,2 \sigma^{\star} \sigma^{*}, 4$ 우 우, leg. R. Hensen.

Remarks: Three provinces are added to the distribution records of $H$.armeniacus. It is noteworthy that although Erzurum is the locus typicus of this species and thousands of Hylaeus specimens have been collected from various parts of the Erzurum province, including Horasan, unfortunately this species has not been found again. The distribution of $H$. armeniacus is concentrated in the eastern and south-eastern parts of the country. The flight season is from May to the end of August. It is only sparsely collected in Turkey (7 provinces).

Distribution: Azerbaijan, Armenia, Iran, Israel, Lebanon; Turkey: Erzurum, Konya, Mersin, Sanlıurfa (WARNCKE 1981); Adıyaman, Ağrı, Tunceli.

\section{Hylaeus (Spatulariella) cypricola (WARNCKE, 1972) Fig. $1 \mathrm{~h}$}

Spatulariella (Brachyspatulariella) dimidiata PitTioni, 1950: 96-102, ơ ㅇ. Cyprus: Limassol.

Prosopis (Spatulariella) cypricola WARNCKE, 1972: 759, nom. nov. for Spatulariella dimidiata Pittioni, 1950 nec. P. dimidiata Perkins, 1899. - Warncke 1985: 58; 1992: 766, 768.

Hylaeus (Spatulariella) cypricola (WARNCKE, 1972) - DATHE 1980: 284. 
Remarks: H. cypricola was originally described from Cyprus. Warncke stated that it is not endemic there, as DATHE (1980) assumed, because it has been reported from several places in northern Egypt so far (WARNCKE 1992: 766). An occurrence on the nearby Turkish mainland is therefore probable, but despite intensive search in Turkey no sample was found for the present study. The species can therefore not (yet) be counted to the Turkish fauna.

\section{Hylaeus (Spatulariella) hyalinatus SMıтH, 1842 Fig. 1b}

Hylaeus hyalinatus SмIтH, 1842: 58, o o $0^{\star}$. Great Britain. Prosopis (Spatulariella) hyalinata hyalinata (Sмітн, 1842) WARNCKE 1972: 758; 1981: 165; 1992: 765.

Hylaeus (Spatulariella) hyalinatus SмIтн, 1842 - DATHE 1980 280; Dathe \& Proshchalykin 2018.

Material examined: Ağrı: Cumaçay, 1930 m, 23.07.1996, 4 우 이 leg. P. Rasmont. Artvin: Yusufeli, Altıparmak, 1200-1700 m, 29.08.1995, 우, leg. P. Hartmann; Yaylalar, 2000-2300 m, 29.08.1995, 3 ㅇ , leg. M. Kraus. Bolu:

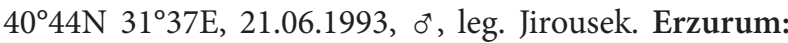
Oltu $20 \mathrm{~km} \mathrm{SW,} \mathrm{07.07.1985,} \mathrm{o*,} \mathrm{M.} \mathrm{Schwarz.} \mathrm{Konya:}$ $25 \mathrm{~km} \mathrm{~N}, 11.06 .1966$, + , leg. H.H.F. Hamann. Sivas: Gürün, 1200 m, 24.07.1986, ㅇ, leg. S.M. Blank.

Remarks: Here, another five provinces are added to the distribution area of $H$. hyalinatus. It has a sporadic distribution. The majority of the specimens was caught in July and August. The species seems to be missing in the Aegean region. It is sparsely distributed in Turkey (6 provinces)

Distribution: In Europe from Iberia to Greece, east to the Caucasus (DAthe et al. 2016); introduced to North America. In Turkey: Erzurum (Özbek 1977); Ağrı, Artvin, Bolu, Konya, Sivas.

\section{Hylaeus (Spatulariella) iranicus DATHE, 1980 Fig. 11}

Hylaeus (Spatulariella) iranicus DATHE, 1980: 93-94, ơ ㅇ. Iran: Damavand (Elburs).

Prosopis (Spatulariella) hyalinata iranica (DAtHe, 1980) WARNCKE 1992: 765, 776.

Material examined: Erzurum: Atatürk University Campus, 2000 m, 11.07.2007, $2 \sigma^{\star} o^{\star}$, leg. J.S. Ascher, H. Özbek, J.G. Rozen (coll. AMNH); Köprüköy, 2,5 km N of Örentaş, 04.07.2004, †, leg. J.S. Ascher, H. Özbek, J.G. Rozen (coll. AMNH); Tortum, 1400 m, 21.07.2010, 2 ㅇ , leg. J.G. Rozen, J.S. Ascher, H. Özbek; Palandöken, 2200-2400 m, 27.07.1986, ㅇ, leg. A.W. Ebmer; Pasinler, $8 \mathrm{~km}$ ENE of Pasinler, 19.07.2010, $2 \sigma^{\star} \sigma^{\star}$, leg. J.S. Ascher, H. Özbek (coll. AMNH). Gümüşhane: Torul, $40^{\circ} 34 \mathrm{~N}$ $39^{\circ} 17 \mathrm{E}, 1000 \mathrm{~m}, 12.07 .1985,6$ 우, leg. M. Schwarz (coll. Schwarz/Ansfelden). Hakkâri: Suvarihalil Geçidi, $2500 \mathrm{~m}, 27.06 .1985,0^{\star}, 2$ 우, leg. M. Schwarz (coll. Schwarz/Ansfelden); Tanin Geçidi, 2200 m, 25.06.1985, $\mathrm{o}^{\star}, 2$ 우 우, leg. C.J. Zwakhals. Kars: Sarıkamış, Karakurt, $20 \mathrm{~km} \mathrm{~W}, 40^{\circ} 10 \mathrm{~N} 42^{\circ} 22 \mathrm{E}, 1600 \mathrm{~m}, 04.07 .1985$, 우, leg. M. Schwarz (coll. Schwarz/Ansfelden). Kahramanmaraş: Göksun, 1400 m, 26.06.1987, ㅇ, leg. Hensen. Konya: Akşehir, Sultandağları, 1800 m, 18.07.1990, 2 ㅇ 우, leg. A.W. Ebmer. Mersin: Mut, Sertavul Geçidi, 1550 m, 08.07.1990, ㅇ, leg. A.W. Ebmer. Nevşehir: Göreme, $38^{\circ} 39 \mathrm{~N} 34^{\circ} 52 \mathrm{E}, 17.07 .1998,20^{\star} 0^{\star}$, 우, leg. C. SchmidEgger; Ürgüp, Topuzdağ1 Geçidi, 1300 m, 17.07.1984, , leg. A.W. Ebmer; Avanos, Zelve, 1050 m, 17.07.1984, + , leg. A.W. Ebmer. Sivas: Gürün, Şuğul Vadisi, 1400 m, 13.07.1990, 3 o $^{\star}$, 우 ; 31.07.1986, 7 o $^{\star} o^{\star}, 3$ ㅇ 우, leg. A.W. Ebmer. Van: Başkale, 2200 m, 10.07.1987, 2 우 우, leg. R. Hensen.

Remarks: H. iranicus has a narrow distribution area, it is only known from Iran and Turkey, and in Turkey previously only from Hakkâri and Kars. The present study adds another eight Turkish provinces to the distribution records. Konya is the most western region of the species. H. iranicus is quite abundant in the province of Erzurum. As a mountain species it occurs at altitudes of 1000-2500 m, and the samples were collected, probably related to this, mainly in July. Although $H$. iranicus is a recently described species, it has been identified from almost all geographical regions of the country except the Aegean and Marmara regions. It is collected moderately in Turkey (10 provinces).

Distribution: Iran; Turkey: Hakkâri, Kars (WARNCKE 1992), Erzurum, Gümüşhane, Kahramanmaraş, Konya, Mersin, Nevşehir, Sivas, Van.

\section{Hylaeus (Spatulariella) irritans DATHE, 1980 Fig. 1e}

Hylaeus irritans Dathe, 1980: 94-95, ․․ Iran: Zoshk.

Prosopis (Spatulariella) alticola WARNCKE, 1981: 162-163, ơ ㅇ. Iran: Elburs.

Prosopis (Spatulariella) irritans DATHE, 1980 - WARNCKE 1992: 776.

Material examined: Ağrı: Eleşkirt, 2200 m, 14.07.1987, ㅇ, leg. R. Hensen. Erzurum: Köprüköy, 3 km NE of Geyikli, 1700 m, 27.07.2010, ơ, leg. J.S. Ascher, H. Özbek (coll. AMNH). Sivas: İmranlı, 40 km E, 1700 m, 15.07.1984, o, leg. A.W. Ebmer.

Remarks: Like H.iranicus, H. irritans was described by DAthe (1980) from Iran. In Turkey, the species had become known from the provinces Erzincan and Hakkâri. The current study adds three more provinces to the distribution records. It is a mountain species that 
occurs at 1700-2800 m altitude. We believe that in order to accurately determine the range of this species, further research should be carried out in the mountain regions of the country, especially above $2000 \mathrm{~m}$. It is collected only sparsely in Turkey (5 provinces).

Distribution: Iran, Turkey: Erzincan, Hakkâri (20002800 m) (DAThe 1980; WarnCKe 1992), Ağrı, Erzurum, Sivas.

\section{Hylaeus (Spatulariella) longimacula (ALFKEN, 1936)} Fig. $1 \mathrm{j}$

Prosopis hyalinata var. longimacula Alfken, 1936: 53, + $0^{*}$. Lebanon: Said Neil, Becaa.

Prosopis (Spatulariella) punctata longimacula Alfken, 1936 WARNCKe 1972: 757; 1981: 189; 1985: 58; 1992: 766.

Hylaeus (Spatulariella) punctatus longimaculus (AlfKeN, 1936) - DATHe 1980: 286.

Hylaeus (Spatulariella) longimaculus (ALFKen, 1936) - DATHE \& Proshchalykin 2018: 79.

John Ascher (Singapore) kindly pointed out to me that the original form "longimacula" is to be interpreted correctly as a noun; it therefore remains unchanged as an apposition (HHD).

Material examined: Adana: Aladă̆, 780 m, 02.07.1985, ㅇ, leg. Y. Barbier. Adıyaman: Kahta, 1700 m, 08.08.1985,

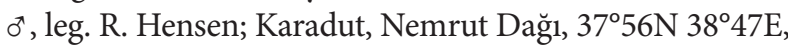
02.07.1993, ơ, leg. Jirousek. Ağrı: Eleşkirt, $30 \mathrm{~km} \mathrm{~W}$, 2200 m, 14.07.1984, ․ Leg. R. Hensen. Aksaray: Hisar, 23.08.2003, ơ, leg. C. Güçlü. Ankara: Kızılcahamam, 29.07.1987, đَ, leg. J. Wimmer; Şereflikoçhisar, 800 m, 23.08.2003, đ, leg. C. Güçlü. Antalya: Alanya, 50 m, 28.07.1985, 10 đ $^{\top}$, 5 우 ㅇ, R. Hensen; Demirtaş, 100 m, 29.07.1985, o , leg. R. Hensen; İncekum, 20 m, 07.08.1985, ơ, P. van Ooijen; Akseki, 1200 m, 09.08. 1985, ơ, 우, leg. P. van Ooijen; Elmall, 24 km S, 1500-1600 m, 04.07.1990, $2 o^{\star} \sigma^{\star}$, + , leg. A.W. Ebmer; Göltarla, 13.07.1996, ơ, leg. Brechtel/Ehrmann; Kemer, Kuzdere, Kesme, 130 m,

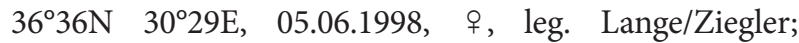
Kuzdere, Kumluca, Arıkanda, 36²8N 3007E, 11.07.1998, $20^{\top} o^{\star}$, + , leg. C. Schmid-Egger; Side, Manavgat, $10 \mathrm{~m}$, 17.06.1987, 2 우 우, leg. R. Hensen; Termessos, 16.07.1983, $\sigma^{7}$, leg. Niehuis. Balıkesir: Burhaniye, 10 m, 18-20.07.1985,

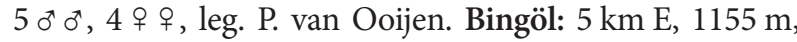
30.05.2002, ㅇ, leg. H. Özbek; Genç, $5 \mathrm{~km} \mathrm{S,} 1400 \mathrm{~m}$, 13.08.1985, ot, leg. R. Hensen. Bitlis: Ahlat, $1750 \mathrm{~m}$, 15.08.1984, ㅇ, 14 우 이 leg. R. Hensen. Bursa: $300 \mathrm{~m}$, 20.07.1987, o ${ }^{\star}$, leg. R. Hensen; Armutlu $15 \mathrm{~km} \mathrm{NE}, 50 \mathrm{~m}$, 27-29.07.1986, 6 o $^{\top}, 2$ 우 우, leg. P. van Ooijen; $20 \mathrm{~km} \mathrm{E}$ Armutlu, 30 m, 20-21.08.1985, ơ , 2 우 ㅇ , leg. P. van Ooijen; Mudanya, 25 m, 25.08.1985, ㅇ, leg. R. Hensen. Diyarbakır: 650 m, 09.08.1985, 4 o $^{\star} \sigma^{\star}$, leg. R. Hensen. Erzurum: Atatürk University Campus, $2000 \mathrm{~m}, 39^{\circ} 53 \mathrm{~N} 41^{\circ} 14 \mathrm{E}, 18.07 .2003$, 2 우 온 leg. J.G. Rozen, H. Özbek; Oltu, Çamlıbel, $22 \mathrm{~km}$ WSW of Oltu, $1700 \mathrm{~m}, 07.07 .2007,2$ ๙ $^{\star}$, ㅇ, leg. J.S. Ascher, H. Özbek, J.G. Rozen; 14 km ENE of Pasinler,
04.07.2007, †, leg. J.S. Ascher, H. Özbek, J.G. Rozen. Eskişehir: Sakari, Ilıca, 06-09.07.1997, 5 o $^{\star} o^{*}, 4$ ㅇ 우, leg. Prudek/Ríha. Gümüşhane: Torul, $800 \mathrm{~m}, 12.07 .1985$, o, leg. C.J. Zwakhals; $1000 \mathrm{~m}, 40^{\circ} 34 \mathrm{~N} 39^{\circ} 17 \mathrm{E}, 12.07 .1985$, 9 우, leg. M. Schwarz. Hakkâri: 35 km E Uludere, 19.09. 1983, ơ, †, leg. Schmid-Egger; Beytüşşebab, Habur

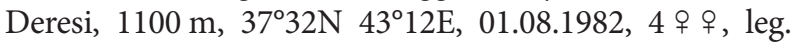
W. Schacht (coll. Schwarz/Ansfelden); Varagöz, $1650 \mathrm{~m}$, $37^{\circ} 25 \mathrm{~N} 44^{\circ} 13 \mathrm{E}, 0^{\star}, 02.08 .1986$, leg. S.M. Blank; Yüksekova $30 \mathrm{~km} \mathrm{W,} 1850 \mathrm{~m}, 19.07 .1986$, ơ, leg. A.W. Ebmer; Sat Dağ $1,1700 \mathrm{~m}, 37^{\circ} 25 \mathrm{~N} 44^{\circ} 13 \mathrm{E}, 4$ o $^{\star} 0^{\star}, 8$ 우 ㅇ , 04-08.08.1983, leg. W. Schacht (coll. Schwarz/Ansfelden). Hatay: Samandağ1 $10 \mathrm{~km} \mathrm{E,}$ 05.07.1998, $5 \sigma^{\star} \sigma^{\star}, 7$ 우, leg. T. Osten. Iğdır: $1000 \mathrm{~m}, 19.08 .1983,4 \sigma^{\star} \sigma^{\star}$, + , leg. J. Timmer. İstanbul: Anadolukavağ 1,30 m, 15.07.1987, ơ, 3 우 우, leg. P. van Ooijen. İzmir: Bergama, $300 \mathrm{~m}, 22.07 .1985,3 \mathrm{o}^{\star} \mathrm{o}^{\star}$, 2 우 ㅇ, leg. R. Hensen. Kahramanmaraş: Göksun, 1400 m, 26.06.1987, ㅇ, leg. R. Hensen. Konya: Beyşehir, 1150 m, 19.08.1987, ơ, ㅇ, R. Hensen; Bozkır, Sarığlan, 1000 m, 25.07.2003, o, + , leg. M. Kesdek. Mersin: Erdemli, $24 \mathrm{~km} \mathrm{NW,} 800$ m, 06.06.1991, ơ, 2 우 ㅇ, leg. S. Kadlec; $20 \mathrm{~km} \mathrm{NW}, 900$ m, 05.07.1996, $2 o^{\star} \sigma^{\star}, 2$ 우 우, leg. Tyrmer/ Vorisek; Göktepedağı, 10.07.1998, đ, leg. M. Riha; Silifke, $35 \mathrm{~km}$ NNW, Kargican, 200 m, 04.08.1985, ơ, 2 우 우, leg. R. Hensen; Uzuncaburç, $400 \mathrm{~m}, 36^{\circ} 34 \mathrm{~N} 33^{\circ} 57 \mathrm{E}$, 20.07.1998, ð’, leg. C. Schmid-Egger; 400 m, 02.08.1985, $3 \sigma^{x} o^{x}, 21$ ㅇ 우, leg. R. Hensen; Kizkalesi, Korykos, 10 m, 03.08.1985, 2 ㅇ ㅇ, leg. R. Hensen. Muğla: Datça, $15 \mathrm{~km} \mathrm{W,}$ 07.10.2007, +, leg. DW Baldock; Fethiye, Ölüdeniz, 26.07.1983, o ${ }^{\top}$, leg. C. Schmid-Egger; Milas, $37^{\circ} 18 \mathrm{~N} 27^{\circ} 57 \mathrm{E}$, 13.07.1998, ơ , 2 ㅇ ㅇ , leg. C. Schmid-Egger. Nevşehir: Acıgöl, Çardak, 38³3N 34²4E, 07.07.1993, ơ , leg. Jirousek (coll. Schwarz/Ansfelden); Kaymaklı, 1200 m, 23.06.1987, , leg. R. Hensen; Ürgüp, $1100 \mathrm{~m}, 11-15.08 .1985,5 \sigma^{\star} \sigma^{\top}$,

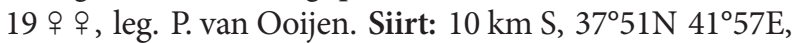
23.06.1985, o`, leg. M. Schwarz (coll. Schwarz/Ansfelden). Sivas: Gürün, Şuğul Vadisi, $1400 \mathrm{~m}, 13.07 .1990,6$ o $^{\star}$, 2 우 ㅇ, leg. AW. Ebmer. Şanlıurfa: Birecik, 06.08.1985, ơ, 2 우 우, leg. R. Hensen; Halfeti, 425 m, 07.08.1985, $5 \sigma^{\star} \sigma^{\star}$, 3 우 ㅇ, leg. R. Hensen (coll. Schwarz/Ansfelden); $400 \mathrm{~m}$, 28.06.1987, 5 우 우, R. Hensen (coll. Schwarz/Ansfelden); $37^{\circ} 15 \mathrm{~N} 37^{\circ} 52 \mathrm{E}, 27.05 .1987$, o ${ }^{\star}$, leg. Madl; $38^{\circ} 30 \mathrm{~N} 43^{\circ} 24 \mathrm{E}$, 03.05.1994, +, leg. D. Kenes. Tunceli: Ovacık, 1250, 19.08.1985, $4 \sigma^{\boldsymbol{}} o^{\boldsymbol{1}}, 9$ 우, leg. R. Hensen. Van: Center, $1700 \mathrm{~m}, 38^{\circ} 30 \mathrm{~N} 43^{\circ} 24 \mathrm{E}, 28.06 .1993$, 우, leg. M. Halada

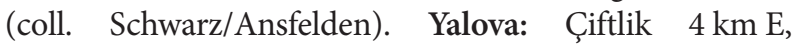
50 m, 31.07.1986, ㅇ, leg. P. van Ooijen. [Kibris, Dipkarpuz, Altınkum, 22.07.2005, $2 \sigma^{\star} \sigma^{*}$, leg. H. Özbek (on Heliotropium europaeum)].

Remarks: Twenty provinces are hereby added to the range of $H$. longimacula. Available records show that this species is one of the most widespread and abundant species in the country. It occurs in various habitats from sea level with warm climate (Antalya, Muğla) to $2200 \mathrm{~m}$ altitude in cool areas (Ağrı, Erzurum), mainly in open fields. Flower visits were observed at Heliotropium europaeum. The flight period is from May to September, the 
species can have more than one generation a year. It is frequently recorded from Turkey (36 provinces).

Distribution: Southern Mediterranean (Morocco to Egypt), Balkan countries, Middle East, Armenia, Azerbaijan, Georgia, Iran. Turkey: Adana, Afyonkarahisar, Antalya, Bursa, Denizli, Hatay, İstanbul, İzmir, Kastamonu, Konya, Kütahya, Manisa, Mersin, Muğla, Samsun, Tekirdağ (WARncke 1972, as Prosopis punctata longimacula), Adıyaman, Ağrı, Aksaray, Ankara, Balıkesir, Bingöl, Bitlis, Dıyarbakır, Erzurum, Eskișehir, Gümüşhane, Hakkâri, Kahramanmaraş, Nevşehir, Siirt, Sivas, Şanlıurfa, Tunceli, Van, Yalova.

\section{Hylaeus (Spatulariella) planulus (WARNCKE, 1981) Figs $15,1 \mathrm{k}$}

Prosopis (Spatulariella) planula WARNCKe, 1981: 167-168, 웅․ Turkey: Hakkâri. - WARNCKE 1992: 768.

Hylaeus (Spatulariella) planulus (WARNCKE, 1981) - comb. nov.

Records: Holotype: ơ Turkey: Pass W Hakkâri in Altin Daglari, 2600-3000 m, 13.08.1979 (leg. Warncke). Paratypes: 1 ㅇ, $10^{\top}$ same location.

Remarks: The face of $H$. planulus is flat as a whole, with the supraclypeal area only slightly raised. This is particularly noticeable in the female, where the supraclypeal field merges broadly and flatly into the forehead. The apical process of sternum 8 of the male is large and threelobed, similar to H. ibex Morawitz, 1877 (see Dathe \& Proshchalykin 2017: 22); however, in contrast the elongated gonoforcipes remain narrow in width.

H. planulus was described from Hakkâri at altitudes of 2600-3000 m. In the present study no further specimens were recorded. This could be due to the fact that $H$. planulus is species of high altitude. We are of the opinion that for such species more effort should be expended in collecting at high mountains. Currently H. planulus appears endemic in Turkey, only known from the type locality (Hakkâri) and represented with three samples $\left(2 o^{x} o^{x}, 1+\right.$ ) ). It is rarely recorded from Turkey (1 province, Fig. 17.11).

\section{Distribution: Turkey: Hakkâri.}

\section{Hylaeus (Spatulariella) punctatus (BRULLÉ, 1832)} Fig. $1 \mathrm{i}$

Prosopis punctata Brullé, 1832: 359, ơ + . Greece: Pamisus valley.

Prosopis (Spatulariella) punctata punctata BRULlé, 1832 WARNCKE 1972: 757; 1992: 766, 768.

Hylaeus (Spatulariella) punctatus (BRULlé, 1832) - DATHE 1980: 285; DATHE et al. 2016.
Material examined: Turkey: 06.08.-20.10.1918, $0^{\top}$, 우 leg. L. Gardener. Adana: Pozant1, 06.07.1983, †, leg. J. Hladil. Antalya: WNW 25 km, Güllük Dağ1, 950 m,

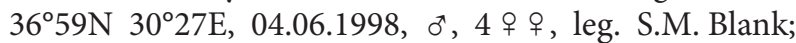
Arapsuyu, Azmak, 5 m, 24.09.2004, 2 ơ $^{\top}, 6$ ㅇ 우, 10 m,

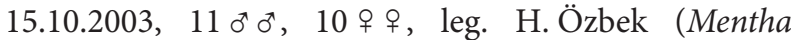
longifolia); Akseki, Cevizli, 03.07.1996, ㅇ, leg. Brechtel/Ehrmann; Altınyaka, 28.05.2009, $1010 \mathrm{~m}$, ơ, leg. J.S. Ascher, H. Özbek, J.G. Rozen; Gündoğmuş, Pembelik, $1090 \mathrm{~m}, 36^{\circ} 83 \mathrm{~N} 32^{\circ} 05 \mathrm{E}, 02.08 .2009$, o, 9 우 우, leg. C. Schmid-Egger (coll. S.-E.); Gelesendra, $1500 \mathrm{~m}$, 02.08.2009, 우, 2 우 우, leg. C. Schmid-Egger (coll. S.-E.); İbradi, $6 \mathrm{~km} \mathrm{SE}, 37^{\circ} 12 \mathrm{~N} 31^{\circ} 55 \mathrm{E}, 31.07 .2009,3$ ㅇ 우, leg. C. Schmid-Egger (coll. S.-E.); Söğütcuma, $1150 \mathrm{~m}$, $36^{\circ} 39 \mathrm{~N} 030^{\circ} 22 \mathrm{E}, 02.06 .1998$, 우 , leg. S.M. Blank; Kemer W $7 \mathrm{~km}$, Kesme, $130 \mathrm{~m}, 36^{\circ} 39 \mathrm{~N}$ 030²9E, 05.06.1998, $6 o^{\top} o^{\top}, 12$ ㅇ ㅇ, leg. S.M. Blank (on Paliurus spina-christi);

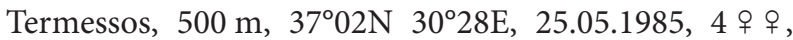
leg. H. Rausch; 24.05.2009, o, leg. J.S. Ascher, H. Özbek, J.G. Rozen (coll. AMNH). Aydın: Kuşadası, 08.05.1992, ${ }^{\star}$, leg. W.H. Liebig. Bilecik: Osmaneli, 27.06.1983, q, leg. J. Hladil. Erzurum: Atatürk University Campus, 2000 m, 13.07.2003, 2 ㅇ ㅇ , 14.07.2003, ㅇ, leg. H. Özbek (on Melilotus officinalis); DSİ Gölet, $1960 \mathrm{~m}, 39^{\circ} 47 \mathrm{~N} 41^{\circ} 09 \mathrm{E}$, 22.07.2005, ㅇ, Narman, Kireçli Dağı, 1950 m, 17.08.2004, † , leg. S. Çoruh; Tortum, Kazandere, 1190 m, 03.08.2004, $\sigma^{*}$, leg. S. Çoruh (on Daucus carota). Hatay: 25.05.1967, $\mathrm{o}^{\star}$, leg. J. Gusenleitner; İskenderun, $20 \mathrm{~km} \mathrm{~S}$ Güzelyayla, 05.07.1996, 웅 leg. Brechtel/Ehrmann; Samandağ1, 27.07.1988, ơ, leg. C. Schmid-Egger. Isparta: Eğirdir, 15.05.1988, ơ, leg. N. Mohr. İzmir: Efes, 18.05.1992, ㅇ, leg. W.H. Liebig. Kars: Pasl1, 11.07.1997, ㅇ, leg. Halada; Sarıkamış, Karakurt, 1500 m, TCK çeşmesi, $40^{\circ} 08 \mathrm{~N} 42^{\circ} 21 \mathrm{E}, 14.08 .2007$, ㅇ, 19.08.2003, $20^{\top} o^{\top}$, 9 우 오, leg. H. Özbek. Kayseri: Develi, Kulpak, $38^{\circ} 27 \mathrm{~N}$ $34^{\circ} 14 \mathrm{E}, 28.05 .2001$, leg. W.H. Liebig. Konya: $25 \mathrm{~km} \mathrm{~N}$, 11.06.1966, ơ, leg. H.H.F. Hamann; Bozkır, Sarığlan, 1000 m, 25.07.2003, $2 \sigma^{\star} o^{\star}$, leg. M. Kesdek; Eflatunpinarı,

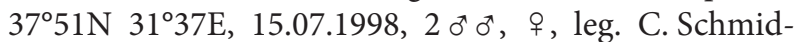
Egger; Güneysınır, Gürağaç, $1020 \mathrm{~m}, 06.08 .2002$, o 2 우 우, leg. M. Kesdek; Seydişehir, 10 km E, 03.07.1996, $\sigma^{*}$, leg. Brechtel/Ehrmann. Mersin: Baland1z, $1000 \mathrm{~m}$,

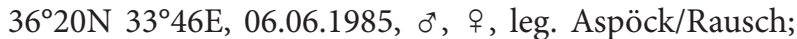
Gülnar, 1000 m, 31.07.1988, 3 o o $^{\star}, 2$ ㅇ ㅇ, leg. C. SchmidEgger (coll. S.-E.); Silifke, Kizkalesi, 09.05.1988, o, leg. N. Mohr; 22.09.1983, ㅇ, leg. C. Schmid-Egger. Muğla:

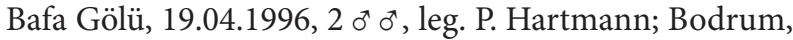
Salmak1s, $37^{\circ} 02 \mathrm{~N} 27^{\circ} 25 \mathrm{E}, 13-24.07 .2001,50^{\star} 0^{\top}, 3$ ㅇ 우, leg.

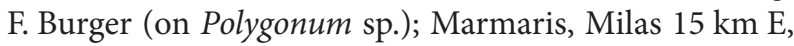
$37^{\circ} 18 \mathrm{~N} 27^{\circ} 57 \mathrm{E}$, o $0^{\star}$, leg. C. Schmid-Egger. Şanlıurfa: Halfeti, 08.06.1983, o ${ }^{\star}$, leg. Schmidt. Van: Muradiye, $1750 \mathrm{~m}$, 16.07.1988, $2 \sigma^{\star} \sigma^{\star}$, leg. C. Schmid-Egger.

Remarks: This study includes $H$. punctatus from another 14 provinces covering all geographical regions except the Black Sea region. It occurs from sea level (Antalya, İzmir) to $2000 \mathrm{~m}$ altitude (Erzurum). The flight season extends from the end of May to mid-October with a peak in June 


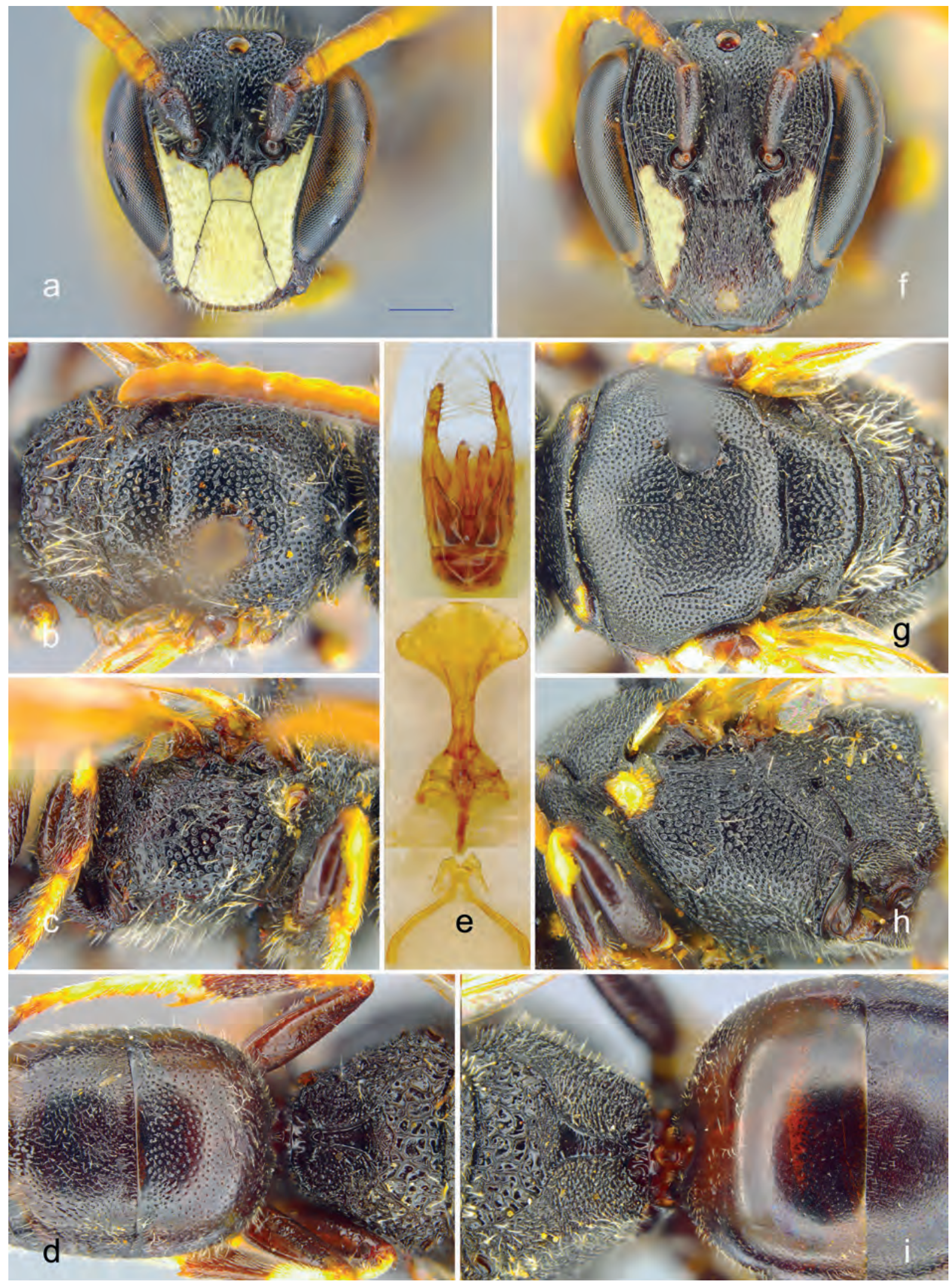

Fig. 15: H. (Spatulariella) planulus (WARNCKe, 1981). Holotype male: a-face, b-mesonotum, c-mesopleuron, d-propodeum and metasoma, e-terminalia. Paratype female: $\mathbf{f}$-face, $\mathbf{g}$-mesonotum, $\mathbf{h}$ - mesopleuron, $\mathbf{i}$ - propodeum and metasoma. - Scale bar $0.5 \mathrm{~mm}$. 


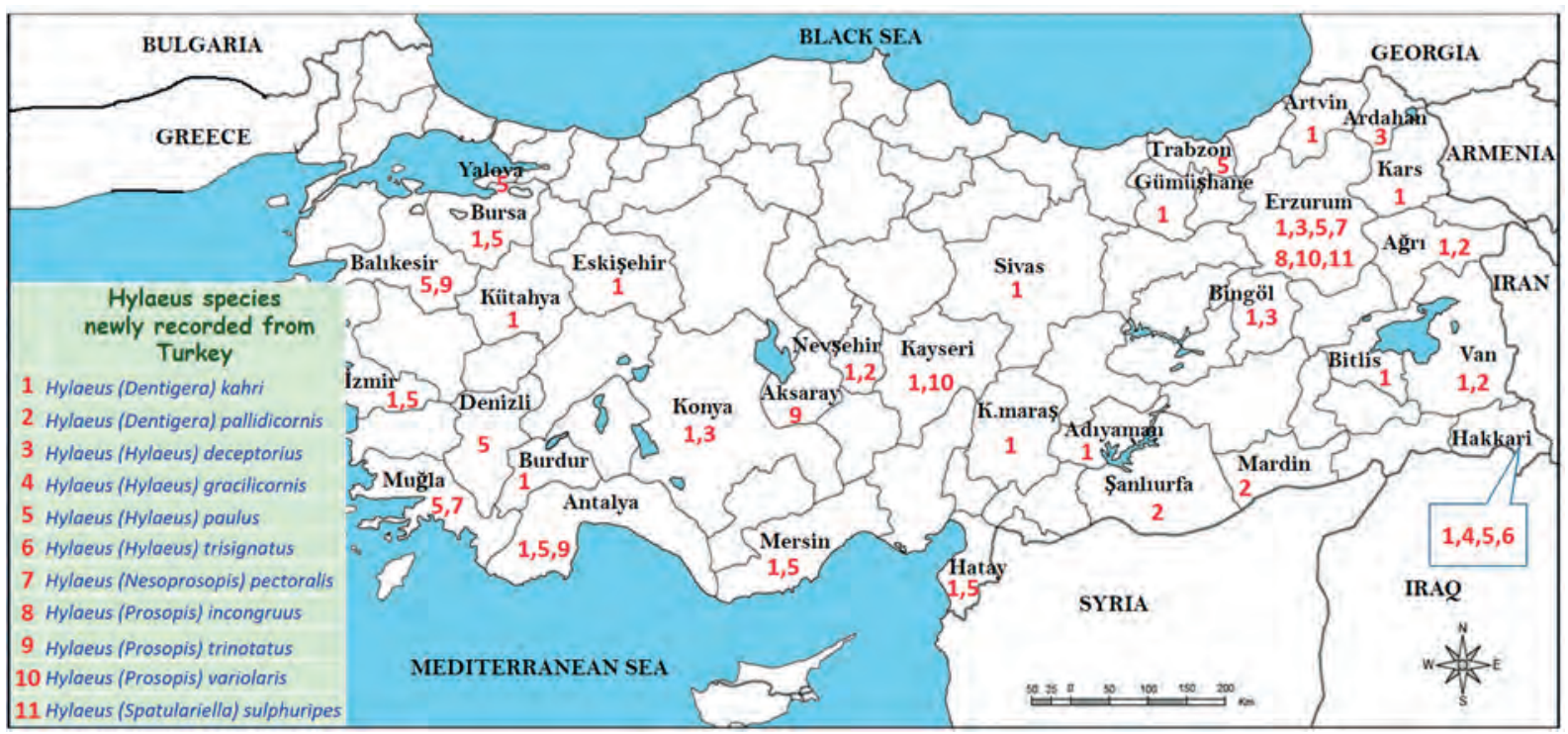

Fig. 16: Distribution map of newly recorded Hylaeus species in Turkey.

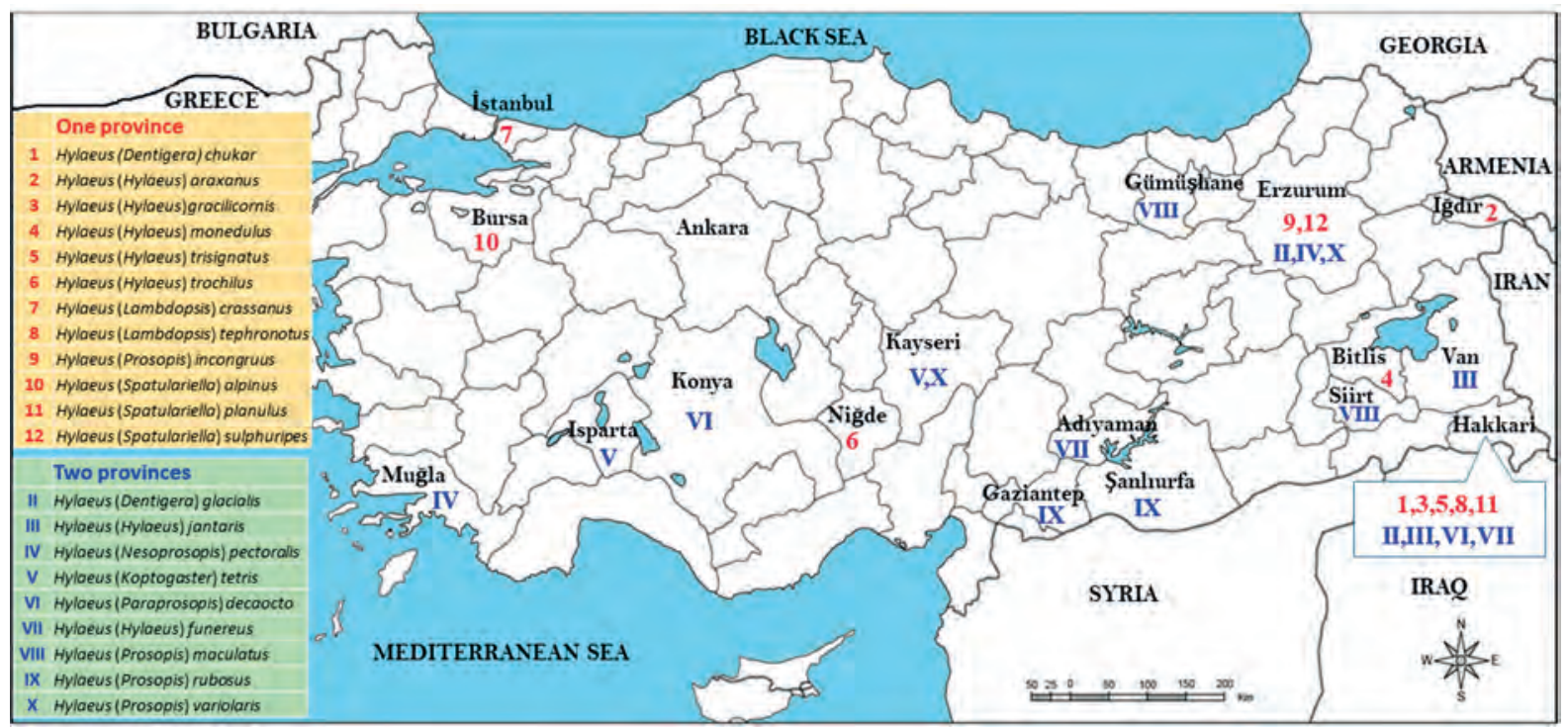

Fig. 17: Distribution map of rarely recorded Hylaeus species in Turkey: species collected in only one (1-12) or two (II-X) provinces.

and July. This suggests that $H$. punctatus has more than one generation. Some specimens have been caught on the flowers of the following plant species: Daucus carota, Melilotus officinalis, Mentha longifolia and Polygonum sp. It is frequently found in Turkey (15 provinces).

Distribution: A European Mediterranean species known from Azerbaijan, Turkey and Ukraine, introduced in North and South America (Sheffield et al. 2011). In Turkey: Erzurum (Özbeк 1977); Adana, Antalya, Aydın, Bilecik, Hatay, Isparta, İzmir, Kars, Kayseri, Konya, Mersin, Muğla, Şanlıurfa, Van. WARncke (1992) noted the species in Turkey without location.
Hylaeus (Spatulariella) sulphuripes (GRIB0Do, 1894) Fig. $1 \mathrm{~g}$

Prosopis sulphuripes GribODo, 1894: 265-266, ㅇ o . Algeria. Prosopis (Spatulariella) sulphuripes GRIBODO, 1894 - WARNCKE 1972: 759; 1992: 766, 768.

Material examined: Erzurum: Palandöken Mt, 2200 m, 16.08.1987, ơ , leg. H. Özbek.

Remarks: H. sulphuripes is hereby for the first time recorded from Turkey as well the Asian continent. Currently, H. sulphuripes is represented by a single sample only. It is rarely recorded in Turkey (1 province, Figs 16.11, 17.12). 
Distribution: Southern West Europe, North Africa. New for Turkey!

\section{Hylaeus (Spatulariella) tauricus (WARNCKE, 1981) Fig. 1d}

Prosopis hyalinata taurica WARNCKE, 1981: 165-166, 우 $0^{*}$. Turkey: Sertavul/Taurus.

Hylaeus (Spatulariella) tauricus WARNCKE, 1981 - comb. nov.

Material examined: Aksaray: Sivrihisar Geçidi, $1800 \mathrm{~m}$, $38^{\circ} 16 \mathrm{~N} 34^{\circ} 19 \mathrm{E}, 03.07 .1984$, ㅇ, leg. A.W. Ebmer. Ardahan: Hanak, 2100 m, 25.07.1986, , leg. A.W. Ebmer. Erzurum: Palandöken, 2200-2400 m, 27.07.1986, $20^{\text {ד }} 0^{\star}$, + , leg. A.W. Ebmer; Pasinler, Calıyazı, 2300 m, 13.07.1997, ơ, leg. E. Ylldırım (on Cephalaria procera); Ügümü, $1600 \mathrm{~m}$, 10.07.2007, ơ, leg. H. Özbek; Tortum-Narman arasi, Kireçli Geçidi, 2100 m, 14.07.1984, ơ, leg. A.W. Ebmer. Kayseri: Erciyas Dağı, 1850 m, 05.07.1984, 2 o $^{\top}$, leg. A.W. Ebmer; Develi Geçidi, 1800 m, 15.07.1990, क , leg. A.W. Ebmer; Bakırdağ 06.07.1984, ơ, 2 우 ㅇ, leg. A.W. Ebmer. Sıvas: Ylldizeli, Çamlıbel Geçidi, 1600-1700 m, 16.07.1984, o, leg. A.W. Ebmer.

Remarks: H. tauricus was first described from Mersin and Karaman as a subspecies of Hylaeus hyalinatus at altitudes of 1200-1300 m (WARNCKE 1981). In the present study it is reported from another five provinces. The species has a sporadic distribution and could be considered as a mountain species occurring between $1200 \mathrm{~m}$ and $2400 \mathrm{~m}$ above sea level. It is only sparsely collected in Turkey (7 provinces).

Distribution: Turkey: Mersin, Karaman (WARNCKe 1981); Aksaray, Ardahan, Erzurum, Kayseri, Sivas.

\section{Discussion}

In this study we list records of 73 species from 10 subgenera of the genus Hylaeus from more than 1600 locations in Turkey. Together with the published records, 86 species were identified for the area. Worldwide, the genus comprises 52 subgenera with about 600 species, of which more than 200 occur in the Palaearctic (Michener 2007; Dathe 2015; Proshchalykin \& Dathe 2017). As we have shown, more than $40 \%$ of the Palaearctic species live in Turkey. For comparison: according to current knowledge, 57 Hylaeus species are recorded from Russia (Proshchalykin \& Dathe 2012; 2017), and 70 species from Central Asia (Kazakhstan, Kyrgyzstan, Tajikistan, Turkmenistan and Uzbekistan) (Dathe \& Proshchalykin 2018), whereby the special importance of this area as a centre for the development of biological diversity has been repeat- edly emphasized (Dathe \& Proshchalykin 2017). Eleven of the 52 Hylaeus subgenera worldwide occur in the Western Palaearctic (Dathe \& Proshchalykin 2018). With the exception of the subgenus Mehelyana SANDHOUSE, 1943, which contains only one very rare species, H. friesei (ALFKEN, 1904), all these subgenera are also found in Turkey. Hylaeus s. str. proves to be dominant with 26 species; Prosopis is already clearly less well represented with 16 species, while Dentigera and Spatulariella each have 12 species, Paraprosopis 8 species, Lambdopsis 5, Koptogaster 3 and Patagiata 2. The subgenera Abrupta and Nesoprosopis are each represented by a single species.

Despite intensive collection efforts for many years throughout the whole country, we could not rediscover 13 of the known species: Hylaeus chukar, H. tephronotus and $H$. planulus were described from Hakkâri (WARNCKE 1981, 1992); H. decaocto from Hakkâri and Konya; H. funereus from Hakkâri and Adıyaman; H. giresunus from Hakkâri and Giresun; H. torquatus from Mardin, known also from Hakkâri and Siirt; H. cervinus from Erzurum and Kars (WARNCKE 1992), but recorded also from Adana (DAthe 2000); H. monedulus from Bitlis; H. trochilus from Niğde (WARNCKE 1992); and H. tetris from Isparta and Kayseri (Dathe 2000) (Fig. 17). It is worth mentioning that apart from $H$. torquatus, $H$. araxanus and $H$. crassanus, the remaining species mentioned above as well as $H$. funereus, $H$. kurdus, $H$. planulus and H. tauricus, are currently known from Turkey exclusively. They may be considered endemic to the country. Interestingly, $H$. araxanus was described after a single male from Iğdır (WARNCKe 1981) (Fig. 17), but no other Turkish specimen has been registered. WARNCKE (1981: 192) mentioned 146 specimens of both sexes as paratypes from Iran, and only recently Dathe \& Proshchalykin (2018) discovered that the species occurs quite frequently in Central Asian countries. H. crassanus has only been recorded from İstanbul (WARNCKe 1972) (Fig. 17).

The presence in Turkey of two species, H. cypricola and $H$. damascenes, is not definitely documented. The first was described from Cyprus and reported from Egypt (WARNCKE 1992), but an occurrence on the nearby Turkish mainland is probable. The second is reported from Syria, Lebanon, Iraq and Iran. WARNCKe (1981, 1985) mentioned the occurrence of this species in southern Turkey, but does not give a locality. According to Ascher \& Pickering (2019) these two species are present in Turkey. Another riddle: a male of $H$. tephronotus is unknown, although the original series comprises nine females.

Of the 73 Hylaeus species here recorded, 11 are new to the Turkish fauna: H. kahri, H. pallidicornis, $H$. deceptorius, H. gracilicornis, H. paulus, H. trisignatus, H. pectoralis, H. incongruus, H. trinotatus, H. variolaris and H. sulphuripes. Of these, H. kahri occurs particularly frequently throughout the country (23 provinces). $H$. paulus is moderately abundant (12 provinces), while three species, $H$. pallidicornis, $H$. deceptorius and 
$H$. trinotatus, were recorded sparsely $(5,4$ and 3 provinces respectively). The rest of them are rare: $H$. pectoralis and $H$. variolaris were found in two provinces, $H$. gracilicornis, $H$. trisignatus, $H$. incongruus and $H$. sulphuripes each in only one province (Fig. 17). With the exception of $H$. trinotatus, which occurs in central and western Anatolia, the other species mainly occur in eastern Anatolia; for example, seven species from Erzurum, four species from Hakkâri, two species from Ağrı, Bingöl and Van. The provinces Adıyaman, Bitlis, Mardin and Şanlıurfa are each represented by a single species (Fig. 17). The eastern part of Turkey can therefore be regarded as a special centre for richness and diversity of Hylaeus species. In Eastern Anatolia, the altitude of the mountains exceeds 2500-3500 m, with numerous narrow valleys and high plains. Overall, it is a biologically rich landscape, which is the main reason that Anatolia, especially its eastern part, has a high biodiversity. Terzo (1998) found a similar situation for the bee genus Ceratina in the Middle East, stressing that "In the western Palearctic region, the eastern part of Turkey, all the species of the subgenus Euceratina occurring in the Near East are present in EastTurkey and not the reverse".

The present data show that the ranges of the Turkish Hylaeus species are variable; 21 species are rarely recorded from one or two provinces, 22 species are sparsely recorded (3-7 provinces), 20 species are moderately (8-17) and 21 species are frequently recorded (18 and more). The rarely recorded species (Fig. 17) H. chukar, H. tephronotus, $H$. planulus are known from the type locality Hakkâri, whereas $H$. araxanus, $H$. trochilus, $H$. monedulus are registered from the type localities Iğdır, Niğde and Bitlis (WARNCKE 1981, 1992). H. araxanus is only represented by one male (holotype), $H$. trochilus by three specimens, and $H$. monedulus by two specimens. Two species are European: H. crassanus was already known from İstanbul (WARNCKE 1972), whereas H. alpinus has been recorded from Bursa in the present study. The occurrence of $H$. trisignatus was previously considered to be restricted to Central Asia (Proshchalykin \& Dathe 2018), but it is interestingly recorded from Hakkâri, represented by one female. The transpalaearctic species $H$. gracilicornis was recorded from Hakkâri. H. incongruus, with a distribution range from Europe to Eastern Siberia, and H. sulphuripes, hitherto known from Southwest Europe and North Africa, are both recorded newly from Erzurum. The first is represented by six specimens, and the second by a single specimen.

Of three species not represented by recent specimens, $H$. decaocto and H. tetris (Fig. 17) are rare species, each represented by two specimens, while $H$. funereus material is available from several collection events. Rare species found again recently in Turkey are the European H. glacialis (from Erzurum and Hakkâri; 5 specimens), the transpalaearctic $H$. pectoralis (from Erzurum and Muğla; 4 specimens), and the Asian species H. maculatus from Gümüşhane (4 specimens) and $H$. variolaris from Siirt, Kayseri and Erzurum (3 specimens). H. jantaris was first discovered in Hakkâri (DATHE 1980) and is currently recorded from Van and Hakkâri; although this species is known from only two provinces, it is quite numerous there.

Some of the frequently recorded species are very common and abundant: H. meridionalis is the most widespread and abundant Hylaeus species, ranging from warmer (seashore, Antalya, Muğla, Yalova) to colder regions (2800 m Erzurum), and mostly in open areas, in 46 provinces. With 41 and 40 provinces $H$. lineolatus and $H$. imparilis follow that species. Both species have Palaearctic distribution areas. In addition, four species were recorded from more than 30 provinces: $H$. cornutus and H. longimacula from 36 provinces, $H$. variegatus and $H$. leptocephalus from 35 and 31 provinces respectively.

Comparing the distribution of Hylaeus species in Turkey, we note that almost all those which were previously unrecorded, or infrequently collected, are high mountain species that live at an altitude of 2000 to $3500 \mathrm{~m}$. We believe that in order to define accurately the distribution of these species, and even detect additional species, further research should be focused on the mountainous regions of the country (above $2000 \mathrm{~m}$ ), especially in Eastern Anatolia, throughout the season.

The following plant species have been observed to be visited preferentially by Turkish Hylaeus species: Achillea millefolium, Ammi visna, Astrodaucus orientalis, Centaurea solstitialis, Daucus carota, Eryngium billardieri, E. campestre, E. creticum, Echium italicum, Euphorbia altissima, Heliotropium europaeum, Hippomarathrum microcarpum, Melilotus alba, M. officinalis, Mentha longifolia, Onobrychis viciifolia, Polygonum sp., Tortilis uranica, and Vitex agnus-castus.

Our investigation still leaves many questions unanswered. Overall, we still know far too little about our bees, including the taxonomic status of numerous species, their distribution, phenology and ecology. The data show many gaps and often only few records in proportion to a vast territory. Nevertheless, we have every reason to emphasize once again the richness of the Turkish fauna, its diversity, and assuredly also its ecological importance. We assume that this first modern inventory is only a beginning, notwithstanding the numerous sites and samples. We therefore suspect the existence of unknown, additional components in this rich biodiversity, which can only be gradually revealed by further, above all targeted investigations. It is an important objective of our present work to encourage this and to provide some assistance.

\section{Acknowledgements}

The authors would like to thank especially the numerous collectors whose enthusiastic work in the first place made this overview possible. Their names are noted in the Records. The colleagues of the Biologiezentrum Linz, 
Oberösterreichisches Landesmuseum, supported us again with the loan of types and substantial other material, especially Martin Schwarz, Esther OcKermüller, Fritz Gusenleitner and Maximilian Schwarz. We also thank İBRAHIM YüCEL ÖzBEK (Atatürk University, Erzurum) who kindly prepared the maps. ANDREW LISTON (SDEI) again carefully re-examined the English and provided guidance on scientific issues. The Senckenberg Gesellschaft für Naturforschung Frankfurt am Main is thanked for generous support of HHD in his taxonomic research. Finally, we sincerely thank the reviewers for their valuable comments and recommendations that helped substantially to improve this article, particularly SABINE SCHOdER (Vienna).

\section{References}

Alfken, J. D. 1904: Neue palaearktische ProsopisArten und -Varietäten (Hym.). - Zeitschrift für Systematische Hymenopterologie und Dipterologie 4: 322-327.

Alfken, J. D. 1905: Die Förstersche Monographie der Bienen-Gattung Hylaeus F. (LATr.) = Prosopis F. und die Prosopis-Sammlung Försters. - Abhandlungen herausgegeben vom naturwissenschaftlichen Verein zu Bremen 18: 108-124.

Alfken, J. D. 1931: Über einige Weibchen der Prosopis variegata-Gruppe. - Deutsche Entomologische Zeitschrift 1930: 176-178.

Alfken, J. D. 1936a: Beitrag zur Kenntnis der Bienenfauna von Persien. - Mitteilungen aus dem entomologischen Verein in Bremen 23 [1935]: 21-24.

Alfken, J. D. 1936b: Neue Prosopis-Arten vom Libanon. - Konowia 15: 51-53.

Aliev, H. A. 1986: A synopsis of the bee genus Hylaeus FABRICIUS 1793 of Soviet Azerbaidjan (Insecta: Hymenoptera: Apoidea: Colletidae). - Senckenbergiana biologica, Frankfurt 66 (4/6): 261-269.

Ascher, J. S. \& Pickering, J. 2019: Discover Life - Bee species guide and world checklist (Hymenoptera: Apoidea: Anthophila). - Available from: http:// discoverlife.org/mp/20q?search=Apoidea (last accessed 12 June 2019).

Benoist, R. 1959: Les Prosopis de France (Hyménoptères Apidés). - Cahiers des Naturalistes, Bull. Natur. Paris., N.S. 15: 75-87.

Bramson, K. L. 1879: Die Hymenoptera mellifera der Umgegend von Jekaterinoslaw. - Bulletin de la Société impériale des naturalistes de Moscou 54: 253-306.

Bridwell, J. C. 1919: Miscellaneous notes on Hymenoptera. With descriptions of new genera and species. - Proceedings of the Hawaiian Entomological Society for the year 1918, Honolulu 4 (1): 109-165.

Brullé, A. 1832: Insectes. In: Expédition scientifique de Morée. Zoologie. - Paris, Levrault 3 (2): 1-29, 64-395, 22 tables.
ÇAlmaşur, Ö. \& ÖzBeK, H. 1999: Erzurum'da ayçiçeği (Helianthus annuus L.)'ni ziyaret eden arı (Hymenoptera, Apoidea) türlerinin tespiti ve bunların tohum bağlamaya etkileri. [Determination of bee species (Hymenoptera, Apoidea) visiting sunflower (Helianthus annuus L.) in Erzurum and their effects on seed binding.] - Turkish Journal of Biology 23: 73-89 (in Turkish with English summary).

Chen, X. \& XU, H. 2009: A key to species of the genus Hylaeus (Hymenoptera: Colletidae) from mainland of China with descriptions of new species and new records. - Zootoxa 1974: 31-50.

Cockerell, T. D. A. 1924: Descriptions and Records of Bees. CII. - Annals and Magazine of Natural History, 14 (9): 274-283.

CurTIS, J. 1831: British Entomology: Being illustrations and descriptions of the genera of insects found in Great Britain and Ireland. - Vol. 8 [1831]: 338-385. London.

Dathe, H. H. 1980a: Die Hylaeus-Arten einer apidologischen Sammelreise in den Iran (Hymenoptera, Apoidea). - Entomologische Abhandlungen, Dresden 43 (5): 77-97.

Dathe, H. H. 1980b: Die Arten der Gattung Hylaeus F. in Europa (Hymenoptera: Apoidea, Colletidae). Mitteilungen aus dem Zoologischen Museum in Berlin 56 (2): 207-294.

Dathe, H. H. 2000: Studien zur Systematik und Taxonomie der Gattung Hylaeus F. (3). Revision der Hylaeus-nivalis-Gruppe in Europa und Klärung weiterer westpaläarktischer Arten (Apidae, Colletinae). - Beiträge zur Entomologie 50 (1): 151-174.

Dathe, H. H. 2010: Studien zur Systematik und Taxonomie der Gattung Hylaeus F. (6). Arten asiatischer Hochgebirge und Anmerkungen $\mathrm{zu}$ weiteren asiatischen Arten (Hymenoptera, Anthophila, Colletidae). - Linzer Biologische Beiträge 42 (1): 43-80.

Dathe, H. H. 2014: Studies on the systematics and taxonomy of the genus Hylaeus F. (8). Revision of the Afrotropic subgenus Hylaeus (Deranchylaeus) Bridwell (Hymenoptera: Anthophila, Colletidae). Zootaxa 3874 (1): 1-84.

DAthe, H. H. 2015: Studies on the systematics and taxonomy of the genus Hylaeus F. (10). New descriptions and records of Asian Hylaeus species (Hymenoptera: Anthophila, Colletidae). - Contributions to Entomology 65 (2): 223-238.

Dathe, H. H. \& Proshchalykin, M. Yu. 2016: The genus Hylaeus Fabricius in Mongolia, an updated species inventory (Hymenoptera: Apoidea, Colletidae). Zootaxa 4121 (4): 351-382.

Dathe, H. H. \& Proshchalykin, M. Yu. 2017: Type revision of Asiatic bees of the genus Hylaeus F. described by Ferdinand Morawitz (Hymenoptera: Apoidea, Colletidae). - Zootaxa 4227 (1): 1-48. - doi. org/10.11646/zootaxa.4227.1.1. 
Dathe, H. H. \& Proshchalykin, M. Y. 2018: The genus Hylaeus FABricius in Central Asia (Hymenoptera: Apoidea: Colletidae). - Zootaxa 4517 (1): 1-91. - doi. org/10.11646/zootaxa.4517.1.

Dathe, H. H.; Scheuchl, E. \& OCKermüller, E. 2016 : Illustrierte Bestimmungstabelle für die Arten der Gattung Hylaeus F. (Maskenbienen) in Deutschland und Österreich. - Entomologica Austriaca, Supplement 1: 1-46. - ISSN 1681-0406.

Eversmann, E. 1852: Fauna Hymenopterologica VolgoUralensis. - Bulletin de la Société impériale des naturalistes de Moscou 25: 1-137.

FAbricius, J. C. 1798: Supplementum Entomologiae systematicae. $2+572$ pp. - Hafniae (Proft et Storch).

FABricius, J. C. 1804: Systema Piezatorum, secundum. Ordines, genera, specie adiectis synonymis, locis, observationibus, descriptionibus: $14,440+30$ pp. Brunsvigae (Reichard).

Förster, A. 1871: Monographie der Gattung Hylaeus F. (LATr.). - Verhandlungen der Zoologisch-Botanischen Gesellschaft in Wien 21: 873-1084.

Friese, H. 1898: Beiträge zur Bienenfauna von Aegypten. - Természetrajzi Füzetek 21: 303-313.

Gibbs, J. \& Dathe, H. H. 2017: First records of Hylaeus (Paraprosopis) pictipes Nylander, 1852 (Hymenoptera: Colletidae) in North America. - Check List 13.3.2116, 1-6. - doi.org/10.15560/13.

Gribodo, G. 1894: Note Imenotterologiche. Nota II. Nuove generi e nuove specie di Imenotteri antofili ed osservazioni sopra alcune specie gia conosciute. - Bollettino della Società entomologica italiana 26: 76-136, 262-314.

ITIS 2020: Integrated Taxonomic Information System (ITIS) on-line database. - Available from: http:// www.itis.gov/citation.html (last accessed 24 January 2020).

Janvier, H. 2012: Comportements d'Abeilles Colletidae (Hymenoptera). Les genres Hylaeus, Chilicola, Colletes, Pasiphae, Policana, Cadeguala, Caupolicana, Lonchopria et Diphaglossa. - Historical reprint of the manuscript of the Muséum National d'Histoire Naturelle, Paris, with annotations by H. H. DATHE; M. Kuhlmann \& C. Villemant (eds). - Entomofauna, Monographie 2: I-VII, 1-181. - ISSN 0250-4413.

JURINE, L. 1807: Nouvelle méthode de classer les Hyménoptères et les Diptères. Hyménoptères, vol. I: 1-319. - Genève, Paschoud.

Kirby, W. 1802: Monographia Apum Angliae. II: 388 pp. - Ipswich.

Ljubomirov, T. \& Yildrim, E. 2008: Annotated Catalogue of the Ampulicidae, Sphecidae, and Crabronidae (Insecta: Hymenoptera) of Turkey. - Pensoft Series Faunistica No. 71: 316 pp.

Magretti, P. 1890: Imenotteri di Siria raccolti dall'Avv. to Augusto Medana R. console d'Italia a Tripoli di Siria con descrizione di alcune specie nuove. - Annali del Museo civico di storia naturale Giacomo Doria, Ser. 2, 9 (29) [1889-1890]: 522-548.
Michener, C. D. 2007: The Bees of the World. XVI + 953 pp., 16 pl. - Baltimore, JoHns Hopkins University Press.

Morawitz, F. 1867: Ein Beitrag zur Hymenopteren-Fauna des Ober-Engadins. - Horae Societatis entomologicae Rossicae 5 (1-3): 39-71.

Morawitz, F. 1872: Ein Beitrag zur Bienenfauna Deutschlands. - Verhandlungen der ZoologischBotanischen Gesellschaft in Wien 22: 355-388.

Morawitz, F. 1874: Die Bienen Daghestans. - Horae Societatis Entomologicae Rossicae 10 (2-4) [1873]: 129-189.

Morawitz, F. 1876: Bees (Mellifera). - In: A travel to Turkestan by the member-founder of the society A. P. Fedtschenko accomplished from the Imperial society of naturalists, anthropologists, and ethnographists on a commission from the generalgovernor of Turkestan K. P. von KaufmanN. Issue 13. Vol. II. Zoogeographical Investigations. Pt V. (Division 7). - M. Stanyukevich's Printing house, Moscow, pp. 161-303 + 3 pls. (Proceedings of the Royal Society of Amateurs of Natural History Sciences, Anthropology and Ethnography, 21 (3)). [in Russian].

Notton, D. G. \& Dathe, H. H. 2008: William Kirby's types of Hylaeus Fabricius (Hymenoptera, Colletidae) in the collection of the Natural History Museum, London. - Journal of Natural History 42 (27-28): 1861-1865.

Nylander, W. 1852: Revisio synoptica apum borealium, comparatis speciebus Europae mediae. - Notiser ur Saellskapets pro Fauna et Flora Fennica Foerhandlingar 2: 225-286.

ÖzBeK, H. 1977: Bees of the family Colletidae (Hymenoptera: Apoidea) in the vicinity of Erzurum. - Atatürk University Journal of the Faculty of Agriculture 8 (2/3): 33-40.

ÖzBEK, H. 2008a: Türkiye'de yonca bitkisini ziyaret eden ar1 türleri ve Megachile rotundata F. (Hymenoptera: Megachilidae). [Bee species visiting the alfalfa plant in Turkey and Megachile rotundata F.]. - Uludağ Arıcılık Dergisi 8 (1): 17-29 (in Turkish with English summary).

ÖzbeK, H. 2008b: Türkiye'de 1lıman iklim meyve türlerini ziyaret eden böcek türleri. [Insect species visiting temperate climate fruit species in Turkey.] - Uludağ Arıcılık Dergisi 8 (3): 94-105 (in Turkish with English summary).

ÖzBeK, H. 2011: Korunga (Onobrychis viciifolia Scop.): önemli bir arı bitkisi. [Sainfoin (Onobrychis viciifolia Scop.): An important bee plant.]. - Uludağ Arıcılık Dergisi 11 (2): 51-62 (in Turkish with English summary).

Özbeк, H. 2018: Çayırüçgülü (Trifolium pratense L.)'nün tozlaşmasında arıların önemi. [The importance of bees in pollination of meadow clover (Trifolium pratense L.).] - Uludağ Arıcılık Dergisi 18 (1): 28-41. - doi.org/10.31467/uluaricilik.427585. 
PAnZeR, G. W. F. 1793-1812: Faunae Insectorum Germanicae Initia oder Deutschlands Insecten, vol. V, 1798.

PÉREZ, J. 1896: Espèces nouvelles de Mellifères de Barbarie. (Diagnoses préliminaires). $64 \mathrm{pp}$. - Bordeaux, Gounouilhou.

PÉREZ, J. 1903: Espèces nouvelles de Mellifères. - Actes de la Société linnéenne de Bordeaux, Procés-verbaux 58: LXXVIII-XCIII, CCVIII-CCXXXVI.

Pittioni, B. 1950: Die westpaläarktischen Arten der Gattung Spatulariella Pop. (Hym. Apoidea, Colletidae). - Bollettino della Società veneziana di storia naturale e del Museo civico di storia naturale 5: 76-113.

Pittioni, B. 1952: Über Variabilität und Verbreitung der westpaläarktischen Arten der Gattung Spatulariella Pop. (Hym., Apoidea, Colletidae). - Zeitschrift der Wiener Entomologischen Gesellschaft 37: 187-204.

Proshchalykin, M. Yu. \& Dathe, H. H. 2012: The bees of the genus Hylaeus Fabricius 1793 of the Asian part of Russia, with a key to species. - Zootaxa 3401: 1-36.

Proshchalykin, M. Yu. \& Dathe, H. H. 2016: Additional records of the genus Hylaeus FABRICIUs, 1793 (Hymenoptera: Apoidea: Colletidae) from Siberia, with description of a new species. - Zootaxa 4105 (4): 301-320. - doi.org/10.11646/zootaxa.4105.4.1.

Proshchalykin, M. Yu. \& Dathe, H. H. 2017: New records of bees of the genus Hylaeus Fabricius (Hymenoptera: Colletidae) in the European part of Russia and North Caucasus. - Proceedings of the Russian Entomological Society, St. Petersburg 88 (2): 61-65.

Proshchalykin, M. Yu. \& Dathe, H. H. 2018: In the footsteps of history: the bees of the genus Hylaeus FABricius (Hymenoptera: Apoidea: Colletidae) collected by V. I. Roborovsky and P. K. Kozlov in Northwest China (1895-1926). - Zootaxa 4434 (3): 573-588. - doi.org/10.11646/zootaxa.4434.3.11.

SAUNDERS, S.S. 1850: Descriptions of two new strepsipterous insects from Albania, parasitical on bees of the genus Hylaeus; with some account of their habits and metamorphoses. - Transactions of the Royal Entomological Society of London 1 (2) [1851]: 43-59, 1 tab.

SCHENCK, A. 1853: Nachtrag zu der Beschreibung nassauischer Bienenarten. - Jahrbücher des Vereins für Naturkunde des Herzogthums Nassau, Wiesbaden 9: 88-307.

SCHENCK, A. 1861: Die nassauischen Bienen. Revision und Ergänzung der früheren Bearbeitungen. - Jahrbücher des Vereins für Naturkunde des Herzogthums Nassau, Wiesbaden 14 [1859]: 1-414.

Schmidt, S.; SChmid-Egger, C.; Morinière, J.; HaszPrunar, G. \& Hebert, P. D. N. 2015: DNA barcoding largely supports 250 years of classical taxonomy: identifications for Central European bees (Hymenoptera, Apoidea partim). - Molecular Ecology Resources 15 (4): 1-16. Wiley Online Library. - doi. org/10.1111/1755-0998.12363.
Schoder, S. 2018: The Hylaeus brevicornis group revisited - an integrative approach to delimit four closely related species of masked bees (Hymenoptera: Apidae). - Master's Thesis. Universität Wien: 43 pp.

Sheffield, C.S.; Dumesh, S. \& Cheryomina, M. 2011: Hylaeus punctatus (Hymenoptera: Colletidae) a bee species new to Canada, with notes on other non-native species. - Journal of the Entomological Society of Ontario 142: 29-43.

Smith, F. 1842: Memoir on the genus Hylaeus, with descriptions of several new British species. Transactions of the Royal Entomological Society of London, Journal of Proceedings, March 7th, 1842: 58.

Sмith, F. 1853: Catalogue of hymenopterous insects in the collection of the British Museum. Apidae 1: 1-197, tab. 1-6, London.

Spinola, M. 1838: Des Hyménoptères recueillis par M. Fischer pendant son voyage en Égypte, et communiqués par. M. le Docteur Waltr. - Annales de la Société entomologique de France 7: 437-546.

Straka, J. \& Bogusch, P. 2011: Contribution to the taxonomy of the Hylaeus gibbus species group in Europe (Hymenoptera, Apoidea and Colletidae). Zootaxa 2932: 51-67.

Terzo, M. 1998: Annotated list of the species of the genus Ceratina (LATREILlE) occurring in the Near East, with descriptions of new species (Hymenoptera: Apoidea: Xylocopinae). - Linzer biologische Beiträge 30 (2): 719-743.

VACHAL, J. 1895: Descriptions de nouvelles espèces du genre Prosopis du contour de la Méditerranée. - Annales de la Société entomologique de France, Bulletin des séances 64: 322-325.

WARNCKE, K. 1972: Beitrag zur Systematik und Verbreitung der Bienengattung Prosopis F. in der Westpaläarktis (Hymenoptera, Apoidea, Colletidae). - Bulletin des Recherches agronomique de Gembloux, New Series 5 [1970]: 746-768.

WARNCKE, K. 1981: Beitrag zur Bienenfauna des Iran 12. Die Gattung Prosopis F., mit Bemerkungen zu weiteren bekannten und unbekannten paläarktischen Arten. - Bollettino del Museo Civico di Storia Naturale di Venezia 31 [1980]: 145-195.

WARNCKE, K. 1986: A contribution to the knowledge of the genus Prosopis (Hymenoptera: Apidae: Colletinae) in Israel. - Israel Journal of Entomology 18 [1984]: 53-61.

WARNCKE, K. 1992: 2. Beitrag zur Systematik und Verbreitung der Gattung Prosopis F. in der Westpaläarktis (Hym., Apidae). - Linzer biologische Beiträge 24 (2): 747-801.

Westrich, P. 2019: Die Wildbienen Deutschlands. 2. Auflage. - Stuttgart: Eugen Ulmer, 824 pp. - ISBN 978-3-8186-0880-4. 